\title{
DEUTSCHE
}

\section{MEDIZINISCHE WOCHENSCHRIFT}

Schriftleitung: Prof. Dr. F. Grosse-Brockhoff Düsseldorf

Prof. Dr. H. Krauss

Freiburg/Br.

Dr. H. Posthofen

Stuttgart

Dr. R. H. Rosie

Stuttgart

Prof. Dr. H. Köbcke

München

91. Jahrgang

1. Halbjahr 1966

Hefte $1-26$

GEORG THIEME VERLAG - STUTTGART 


\section{Inhaltsverzeichnis}

\section{Originalarbeiten}

\section{Arzneimittel, Vergiftungen}

Entzündungsstoffe (Frimmer, M.) 39

Nachweis von Barbitursäure-Derivaten im Harn (F r a h m, M.) 81

Die medikamentöse Therapie der Epilepsie (H e s S, R.) 126

Kaliumhaltige Arzneimittel und stenosierende Dünndarmgeschwüre (W e $11 \mathrm{~m}$ a n $\mathrm{n}, \mathrm{K}$. F.) 131

Beeinflußt Alkoholgenuß die Kalkstickstoffvergiftung? ( $\mathrm{H}$ o s c h e k , R.) 138

Uber die Wirkung von adrenergen$\beta$-Rezeptorenblockern auf ventilatorische Funktionen bei obstruktiven Lungenkrankheiten ( $\mathrm{M}$ e i e r, J., Lydtin, H., Z ölln er, N.) 145

Ampicillin-Spiegel im Serum u. Harn bei eingeschränkter Nierenfunktion (Höffler, D., Stege mann, I., S ch el e r, F.) 206

Vergleichende Untersuchungen über die blutdrucksenkende Wirkung von $\alpha$-Methyl-Dopa und $\alpha$-Methylm-tyrosin (Holt m e ier, H. J., v. $\mathrm{Kl}$ e in -Wis en berg, A., Marong i u, F.) 198

Gibt es, außer dem eigenen Willen, wirksame Nicotin-Entwöhnungsmittel? (K u s c h in s ky, G.) 233

Hochdosierte intermittierende Methotrexat-Therapie bei akuten Leukosen im Kindesalter (Bläker, F., L a n d b e c k, G.) 245

Pimaricin: Ein neues auf Pilze wirksames Breitspektrum-Antibiotikum (Experimentelle $u$. klinische Untersuchungen) (Fegeler, F., Biess, B., Nolt in g, S.) 250

Die Entgiftung von Pharmaka (R e m $\mathrm{m} \mathrm{e} \mathrm{r,} \mathrm{H.)} 289$

Die Insulinresistenz ( $\mathrm{Pf}$ e if $\mathrm{f}$ e $\mathrm{r}, \mathrm{E}$. F.) 314

Intravenöse Grippetherapie mit 0,01 g Cyanquecksilber? (L e n d le, L.) 325

Kann nach dem Stand des heutigen Wissens Chininmercuribisulfat zur Prophylaxe bei Geschlechtskrankheiten empfohlen werden? (B r e h m G.) 378

Uberempfindlichkeit gegen Progesteron bei Prurigo mit prämenstrueller Exazerbation ( $\mathrm{H}$ o is $\mathrm{ch}$ e n, W., S t e i g l e d e r, G. K.) 398

Zur Frage der Magenspülung bei Vergifteten (B a l zereit, F., Ar n o ld, W.) 485

Corticosteroide und Keratomykosen (B ö k e , W.) 505

Welche Bedeutung haben heute die zentralwirkenden Analeptika in der Behandlung von Schlafmittelvergiftungen? ( $\mathrm{N}$ e u h a u s, G. A.) 514
Prophylaktische Behandlung mit Zytostatika nach Krebsoperationen? (Bernd $t, H$.) 514

Die medikamentöse Behandlung des Parkinson-Syndroms (K a e s e r, H. E.) 550

Wann und in welcher Dosierung ist eine intravenöse Eisentherapie indiziert? (G e hrman n, G.) 561

Zur thrombolytischen Therapie des frischen Herzinfarkts. I. Einführung, Behandlungspläne, allgemeine klinische Ergebnisse (Schmutz ler, R., Heckner, F., Körtge, P., van de Loo, J., Pezold, F. A. Poliwoda, H., Praetorius F., Z e k orn, D.) 581

Arzneimittel und Apothekenzwang S c h m elcher, R.) 608

Arzthaftpflicht bei Narkoseschäden durch intraarterielle Injektion von Estil (S c h m el c h e r, R.) 665

Uber das cholinergisch-toxische Psychosyndrom prostigminbehandelter Myastheniker (A b e l, M., K o h l m e y e r, K.) 699

Therapie der Mykosen in der inneren Medizin (W e g m a n n, T.) 713

Ist eine Nicotinsäuretherapie peripherer Durchblutungsstörungen vertretbar, wenn gleichzeitig stenokardische Beschwerden bestehen? (K l e p z i g, H.) 725

Kann Dauertherapie mit 16-24 mg Methylprednisolon täglich bei $M$. Addison ein Ulcus duodeni verursachen? (W e is s b e c k e r , L.) 725

Beitrag zur Pathogenese der Insulinresistenz (D it s ch une it, H., Fed e rli n, K.) 853

Werden die einzelnen BiguanidPräparate unterschiedlich vertragen? (M e hn er t, H.) 873

Bestehen Erfahrungen in der Anwendung von Corticosteroiden bei Höhenkrankheit beim Bergsteigen? (S c h w a l b , H.) 873

Ausgedehnte Lungenaffektionen bei scheinbar leichten Intoxikationen durch Nitrosegase (Fritze, E. H a m mer, C. H., We rner, K.) 899

Stellung und Wert der Arsen-Behandlung aus dermatologischer Sicht (P e t z o ld, D.) 909

Uber die Wirkung eines neuen Depot-Biguamids auf den Kohlenhydrat- und Eiweißstoffwechsel bei Diabetes mellitus ( $M$ ü t in g, D.) 939

Werden die einzelnen BiguanidCortisontherapie auf den Augeninnendruck? (H o ll w i c h, F.) 966

Klinik der Insulinresistenz (D a w e $\mathrm{k} \mathrm{e}, \mathrm{H}$.) 973

Penicillin-Allergie (d e Weck, A L.) 999
Kann versehentliche intravenöse Injektion von Citrat bei der Blutentnahme zur Blutsenkungsreaktion nach fünf Stunden einen tetanischen Anfall auslösen? ( $\mathrm{K}$ u s c h in s k y, G.) 1004

Uber die Anwendung von Oxacillin in hohen Dosen und von Amphotericin $B$ bei akutem Nierenversagen ( $\mathrm{S} \mathrm{ch} \mathrm{röd} \mathrm{e} \mathrm{r,} \mathrm{E.,} \mathrm{O} \mathrm{h} \mathrm{m,} \mathrm{H.}$ G., D e u p m a n n, F. J.) 1035

Was ist über gedächtnisfördernde u. -hemmende Substanzen bekannt? (v. B a u m g a r t e n, R.) 1060

Führt längere Einnahme von Diphenylhydantoin zu gastrointestinalen Störungen wie Diarrhoen oder Duodenalulzera? (D r ube, H. Chr.) 1061

Immundepressive Wirkung von Thalidomid? (L e n z, W.) 1132

Sind nach längerer kontrazeptiver Therapie und Ovulationshemmern eine erhöhte Konzeptionschance und eine Häufung von Mehrlingsgeburten zu beobachten? (D ör i n g, G. K.) 1134

Bemerkungen zur Frage der Indikationen und der sogenannten Entgiftung des Streptomycins (Dihydrostreptomycin) (K u schinsky, G.) 1150

Zur Behandlung bakterieller Infektionen mit Kombinationspräparaten von Streptomycin bzw. Dihydrostreptomycin und Penicillin ( $\mathrm{N}$ a u m a n n, P.) 1152

Bemerkungen zur Frage der Penicillin-Streptomycin-Kombinationen aus hals - nasen-ohrenärztlicher Sicht (D e c h e r, H.) 1158

\section{Atmungsorgane}

einschließlich Lungentuberkulose

Respiratory-syncytial-Virus-Infektionen im Kindesalter. Virologische Untersuchungen (S chneweis, K. E., Käckell, M. Y., Nolt e, R., Sinapius, D., Brandis, H.) 53

Zur Therapie des chronisch obstruktiven Lungenemphysems ( $\mathrm{B} \ddot{\mathrm{u}} \mathrm{h} \mathrm{l}$ $\mathrm{m}$ a $\mathrm{n} n, \mathrm{~A}$.) 82

Therapie der Herz- und Lungenveränderungen bei Wirbelsäulendeformierung (H e y $\mathrm{m}$ e $\mathrm{r}, \mathrm{A}$.) 138

Uber die Wirkung von adrenergen $\beta$ Rezeptorenblockern auf ventilatorische Funktionen bei obstruktiven Lungenkrankheiten (M e i e $\mathrm{r}$, J. Ly d t in, H., Z ö 11 n er, N.) 145 
Respiratory-syncytial-Virus-Infektionen im Kindesalter. Klinik u. pathologische Anatomie (S ch n e w e is, K. E., Käckell, M. Y., Nolte, R., Sinapius, D., Brandis, H.) 153

Zur pathologischen Anatomie der Pneumonieformen in der Perinatalperiode (W ö c k e l, W.) 273

Die Lungenszintigraphie als Mittel zur Früherkennung des Lungenkrebses (O e s e r, H., Ernst, H.) 333

Uber die diagnostisch gezielte Gewebspunktion bei unklaren Lungen-Pleura- und Mediastinalprozessen. Bemerkungen zum Beitrag von R. Hauser in dieser Wochenschrift 90 (1965), 1809 (S a n d rit te $r, W$., Ebn e r, H.) 376

Uber die diagnostische gezielte Gewebspunktion bei unklaren Lungen-, Pleura- und Mediastinalprozessen. Schlußwort ( $\mathrm{H}$ a u s e r, R.) 376

Erkrankungen des Respirationstraktes durch Mycoplasma pneumoniae (W i tzle b, W., S p rössig, M., A n g e r, G., H e i d l e r, S.) 429

Zur Notwendigkeit der BCG-Schutzimpfung - Beobachtungen bei umschriebenen Häufungen von Tuberkulose (Truckenbrodt, $H_{\text {., }}$ Hirs chmann, E.) 479

Bourneville-Pringlesche Phakomatose mit Situs inversus, Doppelniere beiderseits und rezidivierendem Spontanpneumothorax (O d y, R., B e r g e r, H.) 488

Sind Pneumotachographen für Untersuchungen der Lungenfunktion in der Praxis zu empfehlen? (U $1 \mathrm{~m}$ er, W. T.) 514

Präoperative und postoperative Lungenfunktionsdiagnostik $(\mathrm{H}$ a r m s, H., R o d e w a ld , G.) 658

Diagnose der Lungenmykose (W e g man n, T.) 711

Wie kann man zuverlässige Lungenfunktionsprüfungen in der internistischen Praxis mit vertretbarem Zeit- und Kostenaufwand durchführen? (H e r z o g, H.) 725

Werden Pleuraergüsse als Folge von Pankreaspseudozysten häufiger beobachtet (H e s s, W.) 783

Hyalin-Membran-Syndrom

(H i r s chmann, E.) 822

Ausgedehnte Lungenaffektionen bei scheinbar leichten Intoxikationen durch Nitrōsegase (Fritze, E. H a m m a r, C. H., Werner, K.) 899

Allergiediagnostik beim Asthma bronchiale (Gron em e yer, W.) 902

Spezifische Desensibilisierung mit Inhalationsantigenen ( $\mathrm{Fuchs}, \mathrm{E}$.) 904

Untersuchungen über Häufigkeit und Bedeutung von Infektionen mit Mycoplasma pneumoniae

(K r e ch, U., Modde, H.) 1013

Wirksamkeit und Risiko der "Grippeimpfungen"? (M a a s s , G.) 1098

Muß bei einem Moro-positiv gewordenen Säugling eine Isoniazid-Behandlung durchgeführt werden, auch wenn dieser normal gedeiht? (S p i e s s, H.) 1171
Pleurodynie und akutes abdominelles Syndrom (A rend, P., Mar t i n i, G. A.) 1180

Behandlung des Lungenödems mit Procain? (B u d e l m a n n, G.) 1202

\section{Augen}

Weisen Xanthelasmen am Augenlid auf eine familiäre Hypercholesterinämie hin? (Braun-Falco, O.) 282

Zur Therapie der Retinopathia diabetica mit Fructose ( $\mathrm{S} \mathrm{Ch} \mathrm{rader}, \mathrm{K}$. E., Mähr, G., N eum ann, P.) 306

Das Gelbsehen - ein seit über 2000 Jahren bekanntes Phänomen (K O b e r, P.) 373

Ist die Credésche Augenprophylaxe bei jeder Geburt gesetzlich vorgeschrieben? (K ö n i g, P. A.) 467

Corticosteroide und Keratomykosen (B ö k e, W.) 505

Welches komplikationsarme Mydriatikum zur Diagnostik ist zu empfehlen? ( $\mathrm{H}$ oll w i c h, F.) 666

Sehschärfenbestimmung durch Optiker keine Ausübung der Heilkunde (S c h m e l c h e r, R.) 720

Wie kann der Augeninnendruck zuverlässig und rasch in der Praxis gemessen werden? (Holl wich, F.) 829

Welchen Einfluß hat langdauernde Cortisontherapie auf den Augeninnendruck ( $\mathrm{H}$ oll $\mathrm{w} \mathrm{i} \mathrm{c} \mathrm{h,} \mathrm{F.)} 966$

\section{Bewegungs- und Stützapparat}

Welchen Einfluß haben verschiedene Sportarten auf juvenile Kyphosen? (R a t h k e, F. W.) 44

Möglichkeiten zur Bestimmung des Skelett-Calciumgehaltes in der Klinik (K r o k ow s k i, E.) 80

Grundlagen zur Behandlung von Venenerkrankungen der Extremitäten mit Kompressionsverbänden ( $\mathrm{H}$ a i d, H.) 73

Therapeutische Möglichkeiten bei progressiver Muskeldystrophie (B e c kmann, R., von Os ten, V.) 108

Therapie der Herz- und Lungenveränderungen bei Wirbelsäulendeformierung? ( $\mathrm{H}$ e y m er, A.) 138

Zur Frühest- und Frühdiagnose der sogenannten angeborenen Hüftgelenksluxation (D ö r r, W. M.) 168

Laboratoriumsbefunde bei chronischentzündlichen Rheumaerkrankungen (Moll, W.) 219

Gibt es unspezifische Erhöhungen des Antistreptolysintiters? (S ü d h of , H.) 282

Kniegelenks- und Hüftgelenkskontrakturen bei narbiger Irritation des sensiblen Astes des Nervus obturatorius ( $\mathrm{F}$ e $\mathrm{t} \mathrm{t}$ w e i s, E.) 313

Therapie nächtlicher Armschmerzen? ( $\mathrm{i}$ i k k e, R.) 327
Das Multifidus-Dreieck-Syndrom (S c h m i t t, A., Ki e n le, G.) 444 Aussagewert eines positiven Rheumafaktors und des positiven C-reaktiven Proteins? ( $\mathrm{S}$ üd h of , H.) 514

Thyreotoxische Myopathie (Kluthe R., Mertz, D.P., Wesle, H. Ve ih e $1 \mathrm{~m}$ a $\mathrm{n} n$, D.) 536

Das Schleudertrauma der Halswirbelsäule und seine verschiedenen Folgen, Differentialdiagnose und Therapie (M ülle r, E.) 588

Uber das cholinergisch-toxische Psychosyndrom prostigminbehandelter Myastheniker (A b e l, M., K o h lm e y e r, K.) 699

Innenohrschwerhörigkeit bei Dystrophia myotonika u. Dystrophia muscularis progressiva ( $\mathrm{Ku} \mathrm{h} \mathrm{n}, \mathrm{E}$. E r g, W.) 947

Ursache und Therapie der Meralgia paraesthetica ( $\mathrm{Mum}$ e $\mathrm{nth}$ a le r M.) 1060

Physiopathologie und Therapie der Osteoporose (H i o c o, D.) 1079

\section{Biographien und Nachrufe}

Johannes Zeissler 1883-1965 (C a s e lit z) 232

Dankwart Ackermann 1878-1965 (K l e n k, E.) 280

Werner Leibbrand zum 70. Geburtstag (S c h u m a c he r, J.) 419

Prof. O. Schaumann zum 75. Geburts$\operatorname{tag}$ (B r ü c k e, F.) 724

Klinische Physiologie. Entwicklung, Organisation und Aufgaben dieses Fachgebietes in Schweden ( $\mathrm{G} \mathrm{r}$ a K., S t r ö m, G.) 911

Professor Dr. Gjöran Liljestrand zum 80. Geburtstag (v. B r ück e, F.) 1059

Wann und aus welchem Anla $B$ wurde die erste Quaräntäne eingerichtet? ( $\mathrm{R}$ a $\mathrm{t} \mathrm{h}, \mathrm{G}$.) 1134

\section{Blut und blutbildende Organe}

Differenzierung der essentiellen familiären Hyperlipoproteinämien (Hyperlipidämien) (K a hlk e, W.) 26

Die thymoiden Organe des Immunitätssystems ( $\mathrm{G}$ ü $\mathrm{n} t \mathrm{~h}$ e $\mathrm{r}, \mathrm{O}$.) 87

Hypofibrinogenämische hämorrhagische Diathese bei Prostatakarzinom (O h l e r, W. G. A., F i s c h e r, J. Enders, W., Kikillus, B.) 119

Auswirkungen transplazentarer Blutzellenpassagen auf das Kind (O e h m e, J.) 129

Ist eine prophylaktische Therapie der Hämophilie A mit Faktor VIII möglich? (D e u t s c h, E.) 186

Ist bei Kreuzproben die Bestimmung der Rhesus-Untergruppen vorgeschrieben? (S p i elmann, W.) 186

Können bei vorübergehend bettlägerigen Patientinnen weiter verordnete Kontrazeptiva eine Hyperkoagulabilität des Blutes bedingen? (K ir c h h of $\mathrm{f}, \mathrm{H}$.) 187 
Welchen Einfluß hat die Einwirkungsdauer und -temperatur der Coffeinlösung bei der Bilirubinbestimmung nach Jendrassik und Cleghorn? (B ü t t n e r, H.) 233

Gibt es gesicherte Fälle von Mononucleosis infectiosa mit konstant negativer Reaktion nach Hanganutziu-Deucher? (V o r l a e nd e r, K. O.) 233

Hochdosierte intermittierende Methotrexat-Therapie bei akuten Leukosen im Kindesalter (B lä k e r, F., L a n d b e c k, G.) 245

Die Bedeutung der Serumlabilitätsproben in der modernen Leberdiagnostik (L i n d n e r, H.) 267

Die Therapie plasmatischer Gerinnungsdefekte. Hereditäre Koagulopathien (Schmutzler, R.) 269

Fibrinolyse und Blutverlust nach Prostata-Operationen und deren Beeinflußbarkeit durch Antifibrinolytika. $\varepsilon$-Aminocapronsäure u. Kallikrein-Inhibitor Trasylol

(Schmutzler, R., Fürsten b e r g, H.) 297

Die Insulinresistenz ( $\mathrm{P} f \mathrm{e}$ if $\mathrm{f}$ e $\mathrm{r}, \mathrm{E}$. F.) 314

Die Therapie plasmatischer Gerinnungsdefekte. II. Erworbene Koagulopathien ( $\mathrm{Schmutzler}, \mathrm{R}$.) 316

Grundlagen der Lymphozytenkultur ( $\mathrm{H} \mathrm{u}$ b e r, H., $\mathrm{H}$ u b e r, C., Braunste in er, H.) 360

Schwere Thrombozytopenie mit hämorrhagischer Diathese bei infektiöser Mononukleose (B r it ti nge $r$, G., He nne, G., Kön i g, E., Masson, M., Stein a cker, H. G.) 400

Klinische Bedeutung der Lymphozytenkultur $(\mathrm{H} \mathrm{u} \mathrm{ber}, \mathrm{H} ., \mathrm{Hu}$ b e $\mathrm{r}$, C., Bra uns te in e r, H.) 413

Uber Häufigkeit und klinische Bedeutung von Autoantikörpern bei Schilddrüsenerkrankungen, insbesondere bei der Hyperthyreose (Uth ge nannt, H., Müller, W., W e in re ic h, J.) 437

Autoimmunhämolytische Erkrankungen ( $\mathrm{H}$ e n n e m a n n, H. H.) 473

Aussagewert eines positiven Rheumafaktors und des positiven C-reaktiven Proteins? (S ü dh of, $\mathrm{H}$.) 514

Die Lymphogranulomatose (G r os s, R., Z a ch, J., Schulten, H.K.) 521

Wann und in welcher Dosierung ist eine intravenöse Eisentherapie indiziert? (G e h r m a n n, G.) 561

Welche serologischen Tests sind zur sicheren, rechtzeitigen Erkennung von Blutgruppenunverträglichkeiten zwischen Mutter und Kind erforderlich? (Spielmann, W.) 666

Enzephalopathie bei Blutkrankheiten (B o d e chtel, G., B or ch ers, H.-G., Koll m a nn s berger, A.) 673

Zur Problematik der perinatalen Blutungsübel (K ü n z e r, W.) 793

Antigenbindung durch zirkulierende Leukozyten bei der verzögerten lokalen Insulinallergie ( $\mathrm{Fe}$ d e $\mathrm{r} l$ i $\mathrm{n}$, K., H e i n e m a n n, G., Gigli, I., Dits chuneit, H.) 814
Untersuchungen zur Haltbarkeit der Ferment-Aktivität im Serum (Amelung, D., Hofmann, L., O t t o, L.) 851

Laboratoriumsuntersuchungen bei Arteriosklerose (W a g e $\mathrm{n}$ e r, H.) 861

Welche Kriterien bei Blut- und Urinuntersuchungen erlauben die differentialdiagnostische Abtrennung v. funktioneller Hyperbilirubinämie, hämolytischem Ikterus und posthepatitischen Leberschäden? (M a r$k$ of $f$, N. G.) 919

Wie soll heute eine Polycythaemia vera behandelt werden? (B e g e m a n n, H.) 1004

Die Differenzierung myeloischer Leukämien (R e $\mathrm{m} y$, D.) 1055

Ist es bei Befunden des klinischen Laboratoriums sinnvoll und notwendig, Normalwerte anzuführen? (B ü t t n e r, H.) 1061

Klinische und immunologische Aspekte des Morbus Werlhof (G e h rma n n, G.) 1069

Reninaktivität im Plasma bei normalem, erhöhtem und erniedrigtem Blutdruck ( $\mathrm{He}$ in tz, R., Lotz, W.) 1074

Uber die Regulation der unveresterten Fettsäuren unter besonderer Berücksichtigung der Prostaglandine (Böhle, E., Ditschuneit, H., Melani, F., Beyer, J., $S$ ch öf fling, K., $P$ f e if fer, E. F.) 1083

Besondere klinische Formen des Thymus-Syndroms (B ü chner, M. Ge h rma n n, G.) 1105

Inwieweit wird die Blutsenkung durch eine Hypercholesterinämie, Hypertriglyceridämie oder ganz allgemein durch Hyperlipämien beeinflußt? (S c het t le r, G.) 1133

Eisenmangelanämie im Kindesalter (K l e i h a u e r, E.) 1162

Muß die Anforderung einer Blutkonserve schriftlich in die hauseigene Blutbank gegeben werden, die auch die Kreuzprobe ausführt? (S p i e lmann, W.) 1171

\section{Chirurgie}

Kann ein Pruritus ani bei Hämorrhoidalleiden oder nach operativer Beseitigung von Fisteln oder Abszessen durch diätetische Maßnahmen beseitigt werden? ( $\mathrm{R}$ o s c h $\mathrm{ke}, \mathrm{W}$.) 45

Ergebnisse der plastischen Korrektur der Aortenstenose ( $\mathrm{Z}$ e n k e r, R., Seidel, W., Finsterer, U., $\mathrm{Kl}$ inner, W.) 8

Indikation zur Rekonstruktion der Beckenarterien bei kombinierten Gefäßverschlüssen ( $\mathrm{W}$ a i b e l, P.) 23

Maßnahmen bei akutem Kreislaufstillstand (H o s s li , G.) 29

Elektrische Defibrillation bei Vorhofflimmern? (E f f e r $t$, S.) 39

Wann sind vasodilatierende Mittel bei Schock therapie indiziert? (A hnefeld, F. W., Frey, R.) 43

Nierenvenenthrombose beim Neugeborenen. Ein kasuistischer Beitrag (T e ixid or, J., Helbig, D.) 57
Grundlagen zur Behandlung von Venenerkrankungen der Extremitäten mit Kompressionsverbänden

(H a i d, H.) 73

Makroradiographie mit Radiophosphor in der Diagnostik des Magenkarzinoms ( $\mathrm{H}$ a f t e r, E.) 90

Ist beim anaphylaktischen Schock die Anwendung von Corticosteroiden neben der sonstigen Schock therapie indiziert? (We r n e $r$, M.) 95

Neuere Methoden in der Diagnostik spinaler Angiome (P i a, H. W., Vogels ang, H.) 173

Behandlung des Cushing-Syndroms ( $\mathrm{Hen} \mathrm{i,} \mathrm{F.)} 174$

Ist bei Kreuzproben die Bestimmung der Rhesus-Untergruppen vorgeschrieben? (Spielmann, W.) 186

Was dürfen wir vom Elektroenzephalogramm erwarten? (S c h a r f e t t e r, Ch.) 223

Fibrinolyse und Blutverlust nach Prostata-Operationen und deren Beeinflußbarkeit durch Antifibrinolytika. $\varepsilon$-Aminocapronsäure und Kallikrein-Inhibitor Trasylol

(S chmutzler, R., Fürsten b e r g, H.) 297

Uber eine seltene Form von Ileus nach Magenresektion ( $\mathrm{K}$ ol e , E. A. v a n D e urzen, W. A.) 303

Ergebnisse bei der Behandlung des Magenkrebses ( $\mathrm{H} \mathrm{e} \mathrm{g} \mathrm{e} \mathrm{m} \mathrm{a} \mathrm{n} \mathrm{n,} \mathrm{G.,}$ Sc h a u di g, H.) 336

Der Krankenhauschirurg ist gegenüber dem Krankenhausträger bei seiner gefahren- und schadensgeneigten Tätigkeit nicht regreßpflichtig (S c h m e l cher, R.) 365

Derzeitiger Stand der Organtransplantation? (B ü c h e r l, E. S.) 377

Myasthenia gravis bei eineiigen Zwillingen (A d l e r, E.) 396

Die Harnpufferanalyse in der Diagnostik und Therapie metabolischer Störungen des Säure-BasenHaushalts (S o m m e r k a m p, H., Kle t t , H.) 403

Kann eine Kahnbeinpseudarthrose ein Jahr nach einem Trauma als Unfallfolge anerkannt werden, wenn Brückensymptome angegeben werden? ( $\mathrm{J}$ ungh anns, H.) 421

Sollen Ganglien im Handbereich konservativ oder operativ behandelt werden? (E h l e r t , H.) 422

Welche Sonde ist zur Tamponade blutender Osophagusvarizen zu bevorzugen? (H a e m merli, U. P.) 422

Rezidivmitralstenosen (I r mer, W., Obladen, H., Delfino, A. J.) 433

Die erkrankte Doppelniere (Sc h l a c h e t z k i , J.) 448

Trotz Zurücklassung eines Fremdkörpers im Operationsgebiet Ablehnung von Schmerzensgeld (S c h m e l c h e r, R.) 463

Darf die Glucocorticoidtherapie einer chronischen Hepatitis oder Leberzirrhose bei nachgewiesenen Osophagusvarizen fortgesetzt werden? (K ü h n , H. A.) 466

Behandlung von Nachblutungen im Bereich von Operationswunden? (K e r n, E.) 466 
Behindert die Varizen-Sklerotherapie eine spätere Operation? (S i g g , K.) 467

Klinik, Therapie und Morphologie der Tumoren der nicht chromaffinen Paraganglien $(\mathrm{H} \mathrm{el} \mathrm{p} \mathrm{a} \mathrm{p,} \mathrm{E.}$ $\mathrm{H}$ e $1 \mathrm{p}$ a p, B.) 493

Keine Arzthaftpflicht bei unterlassener Antitetanusinjektion (S c h m elcher, R.) 558

Das Schleudertrauma der Halswirbelsäule und seine verschiedenen Folgen, Differentialdiagnose und Therapie (M ü ll e r, E.) 588

Besondere Aufklärungspflicht bei Kropfoperationen (Schmelcher, R.) 608

Anästhesisten als Kassenärzte (S c h m e l c h e r, R.) 608

Kann ein Achillessehnenriß durch ein einmaliges Trauma ohne Vorschädigung entstehen ( $\mathrm{J} u \mathrm{ng}$ h a n $\mathrm{n}$, H.) 611

Experimentelle Chirurgie im Rahmen der Gesamtchirurgie ( $\mathrm{Z} \mathrm{u} \mathrm{k} \mathrm{-}$ s c h w erd t , L.) 617

Krankheiten des chirurgischen Fortschrittes ( $\mathrm{N}$ i s s e n, R.) 622

Morphologie, operative Behandlung und deren Ergebnisse bei 139 Sinus-venosus-Defekten (D e r r a E., I r m e r, W., Tarbiat, S.) 627

Zur Kenntnis eines seltenen, durch ein Pankreasadenom verursachten Krankheitssyndrom ( $\mathrm{Z}$ e n k e r, R. Forell, M. M., Erpenbeck, R.) 634

Die chirurgische Behandlung der Angina pectoris (S e n n i n g, $\AA$.) 641

Zur Frage der konservativen oder operativen Behandlung von Pankreasfisteln ( $\mathrm{K}$ ü m m e rle, F. $\mathrm{M}$ a p p e s, G.) 643

Selektive Vagotonie und Pyloroplastik in der Behandlung des Gastroduodenalulkus und der Gastritis haemorrhagica (A llg ö w e r, M., $\mathrm{H}$ e g g l i n, J.) 648

Präoperative und postoperative Lungenfunktionsdiagnostik ( $\mathrm{H}$ a r m s , H., R o d e w a ld, G.) 658

Therapie der Papillenstenosen ( $\mathrm{H}$ e s s, W.) 660

Therapie der Papillenstenosen (H e s s, W.) 660

Die operative Prophylaxe der zerebralen Mangeldurchblutung (D orndorf, W.) 662

Stationäre Einweisung Unfallverletzter in ein nicht als Unfallkrankenhaus zugelassenes Krankenhaus (Schmelcher, R.) 664

Arzthaftpflicht bei Narkoseschäden durch intraarterielle Injektion von Estil (S c h m e l c h e r, R.) 665

Rezirkulationsperitonealdialyse (B a u m , P., P a b s t , K.) 706

Ergebnisse und Probleme der Nierentransplantation (W o l f f, H. P.) 738

Konservative Therapie bei erhöhtem Hirndruck ( $\mathrm{H}$ e $\mathrm{m}$ m e r, R.) 770

Werden Pleuraergüsse als Folge von Pankreaspseudozysten häufiger beobachtet? (H e s s, W.) 783

Alterschirurgische Probleme bei arteriellen Verschlußkrankheiten (L o o s e , K. E.) 812
Injektionsbehandlung des Hämorrhoidalleidens? (R o s chke, W.) 829

Störungen nach Magenresektion (He n n in g, N., B e r g, G., $W$ üs $t, H ., Z$ e it l e r, G.) 843

Der chronische Verschluß von Gliedmaßenarterien. Durchblutungsförderung (W i d m e r, L. K.) 863

Angiographie bei Beckenarterienverschlüssen zur Indikationsstellung für rekonstruktive Gefäßoperationen (B e r g h u s, H. $\mathrm{Kauf}$ m a n n, H.) 894

Differentialdiagnose und operative Behandlung der spinalen Apoplexie ( $\mathrm{P}$ i a , H. W.) 925

Darmverschluß im Alter ( $\mathrm{H}$ a $\mathrm{ng}$ o s G., Thurzó, R., Csiffary, D.) 952

Sind für eine Tetanus-Grundimmunisierung zwei oder drei Impfungen mit einem Aktivimpfstoff $\mathrm{zu}$ empfehlen? (R o e m e r, G. B.) 966

Erfahrungen mit sterotaktischen Eingriffen bei Epilepsie (Orthner. H., L o h m a n n, R.) 984

Die solitäre Exulceratio simplex (Dieulafoy) als Ursache massiver Intestinalblutungen ( $\mathrm{t} \mathrm{r}$ e i c h e r , H.-J.) 991

Die chirurgische Behandlung der Colitis ulcerosa (D e u c h e r, F.) 1001

Hals-nasen-ohren-ärztliche Erfahrungen mit der operativen Kälteanwendung ( $\mathrm{W}$ ulls t e i n, H. Ger l a ch, J., S pule r, H., Z e h m S.) 1016

Beitrag zur Diagnostik der inneren Hirnvenenthrombose (F i l i p p a , G., Regli, F., Yasargil, M. G.) 1025

Differenzierung verschiedener Formen des Schocks durch einfache Meßverfahren (L u t z , H.) 1043

Indikationen und Grenzen der Gastroskopie (Ot t e nj a n n, R.) 1045

Die operative Wiederherstellung der weiblichen Fertilität (D ö $\mathrm{r}$ i n g , G. K.) 1047

Differentialtherapeutische Erörterungen bei Rückenmarks- und Caudaverletzungen (L a s b e r g , G.) 1109

Ulcus ventriculi dissecans (D o b r o wols ki, J., Golab, W., Szy s z k o, St.) 1121

Die sekundären morphologischen Veränderungen des Gehirns nach Verletzung durch stumpfe Gewalt (M ü $1 \mathrm{l}$ e r, N.) 1126

Muß die Anforderung einer Blutkonserve schriftlich an die hauseigene Blutbank gegeben werden, die auch die Kreuzprobe ausführt? (S pielma n n, W.) 1171

Lymphangioadenographie des retroperitonealen Lymphsystems (B e l t z , L., T h u r n , P.) 1177

Pleurodynie und akutes abdominelles Syndrom (A rend, P., M a r . t i n i, G. A.) 1180

Zur Diagnostik und Therapie der epiduralen Blutung (P oth e, H.) 1186

Wird eine Cholezystektomie bei therapieresistenten, chronisch entzündlichen Gallenblasen angeraten? (K a i s e r, E.) 1205

\section{Ernährung, Diätetik}

Kann ein Pruritus ani bei Hämorrhoidalleiden oder nach operativer Beseitigung von Fisteln oder Abszessen durch diätetische Maßnahmen beseitigt werden? ( $\mathrm{R}$ o s c h $\mathrm{k}$ e, W.) 45

Wie sind die Erfolgsaussichten der Evers-Diät bei multipler Sklerose? (W e l t e, E.) 421

Welche Zucker sind physiologisch am wichtigsten? (M e h n e r t, H.) 467

Sind diätetische Maßnahmen bei Herzinfarkt heute noch nützlich? (S c h e t t le r, G.) 703

Ist es ärztlich vertretbar, Milch aus eigenem, gesundem (Tuberkuloseund Brucellose-freiem) Stall ohne Abkochen oder Pasteurisieren abzugeben? (E y e r , H.) 919

Zur diätetischen Cystinosebehandlung (B a u e $r$, B.) 1124

Wie hoch ist der Methionin. und Cholingehalt von Quark (Leberdiät)? (No e $11 \mathrm{e}, \mathrm{H}$.) 1134

Welche Diät ist bei akuter Glomerulonephritis im Kindesalter zu empfehlen? (D i t t r i c h) 1202

\section{Geburtshilfe und Frauenheilkunde}

Zur Beeinflußbarkeit von Choriokarzinomzellen durch Zytostatika und Immunkörper in der Gewebekultur ( $\mathrm{H}$ e c k m a n n, U., T ra n e k je r, S.) 66

Uber einen neuen glaubwürdigen Fall von Unterschreitung der gosetzlichen Empfängniszeit (D ö r i n g, G. K.) 116

Auswirkungen transplazentarer Blutzellenpassagen auf das Kind (O e h m e , J.) 129

Können bei vorübergehend bettlägerigen Patientinnen weiter verordnete Kontrazeptiva eine Hyperkoagulabilität des Blutes bedingen? ( $\mathrm{K} \mathrm{ir} \mathrm{ch} \mathrm{h} \mathrm{of} \mathrm{f,} \mathrm{H.)} 187$

Amniozentese $u$. intrauterine Transfusion: neue Wege der Behandlung der Neugeborenenerythroblastose (S a c h t le be n, P.) 229

Nochmals: Zum Recht der Schwangerschaftsunterbrechung. Eine notwendige Ergänzung ( $\mathrm{K}$ o h l h a a , M.) 277

Ursachen der 6-Phosphogluconat-Dehydrogenase-Aktivität im Vaginalsekret bei Collumkarzinom (A b r a h a m, R., Vorherr, H.) 345

Uberempfindlichkeit gegen Progesteron bei Prurigo mit prämenstrueller Exazerbation ( $\mathrm{H}$ o is chen, W., St ei gled e r, G. K.) 398

Die plazentare Transfusion (S c h e n c k, W.) 458

Ist die Credésche Augenprophylaxe bei jeder Geburt gesetzlich vorgeschrieben? (K ö n i g , P. A.) 467

Mechanismus, Zeitpunkt und Häufigkeit der Entwicklung einer Rh-Inkompatibilität? (M a r i u s , G.) 610 
Welche serologischen Tests sind zur sicheren, rechtzeitigen Erkennung von Blutgruppenunverträglichkeiten zwischen Mutter und Kind erforderlich? ( $\mathrm{Sp}$ i e l m a n n, W.) 666

Sind Quecksilberschmierkuren zur Prophylaxe der Rubeolenembryopathie zu empfehlen? (Mayer, H.) 919

Wie behandelt man eine Mastodynie? (D ö r in g, G. K.) 1005

Die operative Wiederherstellung der weiblichen Fertilität (D ö r i n g, G. K.) 1047

Chromosomenanomalien als Abortursache (P a r lowitzki', I. H.) 1094

Ist die Bezeichnung "Ovulationshemmer" nach den heutigen Kenntnissen über die Wirkung der Kontrazeptiva noch gerechtfertigt (D ö $\mathrm{r}$ in g, G. K.) 1098

Ikterus als Symptom einer Spätgestose (Mü h l e r, E.) 1114

Sind nach längerer kontrazeptiver Therapie mit Ovulationshemmern eine erhöhte Konzeptionschance und eine Häufung von Mehrlingsgeburten zu beobachten? (D ö r i n g, G. K.) 1134

\section{Geschwülste}

Zur Beeinflußbarkeit von Choriokarzinomzellen durch Zytostatika und Immunkörper in der Gewebekultur (H e c k ma n n, U., T ran ek jer, S.) 66

Makroradiographie mit Radiophosphor in der Diagnostik des Magenkarzinoms ( $\mathrm{H}$ a f t e $\mathrm{r}$, E.) 90

Wie kann man im Saugbiopsiematerial die Tumorzellen von störenden Erythrozytenbeimengungen isolieren, ohne die phasenkontrastzytologische Beurteilbarkeit zu beeinträchtigen? (S e i f e r t , G.) 95

Hypofibrinogenämische hämorrhagische Diathese bei Prostatakarzinom (O h l e r, W. G. A., Fi s c h e r, J., Enders, W., Kikillus, B.) 119

Karzinogenese im Lichte der Statistik. Bemerkungen zum Beitrag von $K$. Freudenberg in dieser Wochenschrift 90 (1965), 944 (S c h i n z, H. R., R e i c h, Th.) 135

Karzinogenese im Licht der Statistik. Schlußwort. (Fre u d e n b e r g, K.) 137

Kindlicher Diabetes mellitus infolge fetaler Inselschädigung? Zur Hypothese von Cornelia van Beck (Schreibenreiter, S., Tha lh a m m e r, O.) 216

Uber ein Lipom der Nebenniere mit Phäochromozytomsymptomatik (L a n g e, H. P.) 254

Die Lungenszintigraphie als Mittel zur Früherkennung des Lungenkrebses (O e s e r, H., Ernst, H.) 333

Ergebnisse bei der Behandlung des Magenkrebses ( $\mathrm{H}$ e g e m a n n, G., $\mathrm{S} \mathrm{ch}$ a u d i g, H.) 336
Die Telecaesiumbehandlung der Larynx - und Hypopharynxkarzinome ( $\mathrm{K}$ a u f $\mathrm{m}$ a $\mathrm{n} \mathrm{n}, \mathrm{H}$.) 339

Ursachen der 6-Phosphogluconat-Dehydrogenase-Aktivität im Vaginalsekret bei Collumkarzinom (A b r a h a m, R., Vorh e r r, H.) 345

Uber die diagnostisch gezielte Gewebspunktion bei unklaren Lungen-, Pleura- und Mediastinalprozessen. Bemerkungen zum Beitrag.v. R. Hauser in dieser Wochenschrift 90 (1965), 1809. (S a ndritter, W., E b n e r, H.) 376

Uber die diagnostische gezielte Gewebspunktion bei unklaren Lungen-, Pleura- und Mediastinalprozessen. Schlußwort ( $\mathrm{H}$ a u s e $\mathrm{r}, \mathrm{R}$.) 376

Hirntumor-Nachweis mit Radioisotopen (S c h n e i d e r, C.) 454

Klinik, Therapie und Morphologie der Tumoren der nicht chromaffinen Paraganglien ( $\mathrm{Hel} \mathrm{p}$ a $\mathrm{p}, \mathrm{E} ., \mathrm{H} \mathrm{el}$ p a p, B.) 493

Therapie des Melanoms (Korting, G. W.) 501

Prophylaktische Behandlung mit $\mathrm{Zy}$ tostatika nach Krebsoperationen? (B ernd t, H.) 514

Die Lymphogranulomatose (G r o s s R., Z a ch, J., Schulten, H.K.) 521

Zur Klinik metastatischer Herzgeschwülste (B r a c h, F.) 593

Zur Kenntnis eines seltenen, durch ein Pankreasadenom verursachten Krankheitssyndrom (Z e n k e r, R., Forell, M. M., Erpenbeck, R.) 634

Paraneoplastischer Hypercorticismus. Klinische, biochemische u. morphologische Befunde ( $\mathrm{H}$ änze，S. P i e r a c h, C. A.) 837

Was leistet die Thormählensche Probe zur Früherkennung des Melanoms? (K a l k of $f, K$. W.) 919

Hals-Nasen-Ohren-ärztliche Erfahrungen mit der operativen Kälteanwendung ( $\mathrm{Wulls}$ sein, $\mathrm{H}$., Gerlach, J., Spuler, H., $\mathrm{Z}$ e h m, S.) 1016

\section{Harnorgane}

Nierenvenenthrombose beim Neugeborenen. Ein kasuistischer Beitrag. ( $\mathrm{T}$ e i x id or, J., Helbig, D.) 57

Hypofibrinogenämische hämorrhagische Diathese bei Prostatakarzinom (O h l e r, W. G. A., F is c h e r, J., Enders, W. Kikillus, B.) 119

Uber die Häufigkeit des pathologischanatomischen Bildes der Schockniere und anderer Nierenerkrankungen im unausgewählten $\mathrm{Ob}$ duktionsgut (S chubert , G. E., $\mathrm{K}$ öberle, H.) 147

Ampicillin-Spiegel in Serum u. Harn bei eingeschränkter Nierenfunktion (H ö f f ler, D., S t e ge m a n n, I., S c h el e r, F.) 206

Welches therapeutische Vorgehen ist bei Libido- und Potenzminderung zu empfehlen? (Klosterhal f e $n, H$.) 234
Fibrinolyse und Blutverlust nach Prostata-Operationen und deren Beeinflußbarkeit durch Antifibrinolytika. $\varepsilon$-Aminocapronsäure u. Kallikrein-Inhibitor Trasylol

(Schmutzler, R., Fürsten b e r g, H.) 297

Derzeit gültige Normgrenzen für Mineralien und andere wichtige Substanzen im 24-Stunden-Harn? (D u l c e , H.-J.) 325

Welche therapeutischen Möglichkeiten bestehen bei Oxalaturie zur Prophylaxe eines Steinrezidivs? (B r o s i g, W.) 326

Uber das infantile nephrotische Syndrom bei kongenitaler Glomerulonephritis (R o s s e n b e ck, H. G., Margraf, O., Hof mann, D.) 348

Die Therapie der Hyperkaliämie (F i li p p i n i, L.) 359

Zur kausalen Pathogenese des infantilen nephrotischen Syndroms bei kongenitaler Glomerulonephritis (R o s s e n b e c k, H. G.) 363

Die Harnpufferanalyse in der Diagnostik und Therapie metabolischer Störungen des Säure-BasenHaushalts (Som me rka m p, H., $\mathrm{Klet} \mathrm{t}, \mathrm{H}$.) 403

Die erkrankte Doppelniere $\mathrm{S} \mathrm{ch} \mathrm{la} \mathrm{ch} \mathrm{et} \mathrm{z} \mathrm{k} \mathrm{i,} \mathrm{J.)} 448$

Therapeutische Möglichkeiten bei paraurethralem Gang? (D e t t mar, H.) 514

Harnkonzentrierung bei essentieller Hypertonie (M e r t z , D. P.) 529

Glomerulonephritis. Aktuelle Gesichtspunkte zur Pathogenese (R o t h e r, Kl.) 551

Welche klinische Bedeutung hat die Bestimmung des Residual-N (nicht Rest-N) (We t z e l s, E.) 562

Probleme der renalen Säure-BasenRegulation (P a b s t, K., G e rok, W., B a u m, P.) 600

Rezirkulationsperitonealdialyse (B a u m, P., P a b s t , K.) 706

Wie erklärt man sich die drohende Niereninsuffizienz schwer Herz,kranker bei zu brüsker Ausschwemmung? (S c hirmeis ter, J.) 726

Ergebnisse und Probleme der Nierentransplantation ( W o lf f , H. P.) 738

Veränderungen der AchillessehnenReflexzeit bei Patienten mit Niereninsuffizienz ( $\mathrm{Fankh}$ a u s e r, S., Morell, B., Reubi, F.) 763

Zur Diagnostik $u$. Therapie von Harnabflußstörungen im Kindesalter Hohenfellner, R., Sökel a n d, J., S tr a u b, E.) 430

Unsere Erfahrungen mit der instrumentellen Chemolitholyse der Nierensteine (B r o s ig, W., Koll. w i t z, A.-A.) 1019

Uber die Anwendung von Oxacillin in hohen Dosen und von Amphotericin $B$ bei akutem Nierenversagen (S c hröder, E., O h m, H. G., D e u p m a n n, F. J.) 1035

Reninaktivität im Plasma bei normalem, erhöhtem und erniedrigtem Blutdruck (Heintz, R., L o t z, W.) 1074 
Die Diagnose der chronischen interstitiellen Nephritis (B e rning, H.) 1090

Welche Diät ist bei akuter Glomerulonephritis im Kindesalter zu empfehlen? (D i t t r i c h) 1202

\section{Haut- und Geschlechtsorgane}

Kann ein Pruritus ani bei Hämorrhoidalleiden oder nach operativer Beseitigung von Fisteln oder Abszessen durch diätetische Maßnahmen beseitigt werden? ( $\mathrm{R} \circ \mathrm{s} \mathrm{ch}$ $\mathrm{ke}, \mathrm{W}$.) 45

Methodische Hinweise zur Diagnostik von Chromosomen-Anomalien (S t o e ckenius, M.) 125

Wie sind in der Haut verbliebene Teile von unsachgemäß entfernten Holzböcken (Ixodes ricinus) zu behandeln? (W e y e r, F.) 186

Ursache einer symptomlosen, rasch sich ausdehnenden, hellweinroten Erythema migrans am Oberschenkel eines Erwachsenen (Korting. G. W.) 233

Fabry-Krankheit (Angiokeratoma corporis diffusum universale) Phosphatidspeicherkrankheit bei zwei Familien

(D u b a c h, U. C., G lo or, F.) 24

Pimaricin. Ein neues auf Pilze wirksames Breitspektrum-Antibiotikum (Experimentelle u. klinische Untersuchungen) (F e g e l e r, F., B i e s $s$, B., Nolt in g, S.) 250

Verfolgungs-Spätschäden auf gynäkologischem Gebiet (D ö r in g, G. K.) 260

Zur Klinik, Pathogenese u. Therapie des Herpes zoster, insbesondere zur Häufigkeit des Zoster duplex unilateralis ( $\mathrm{H} \mathrm{e} 11 \mathrm{e}, \mathrm{S}$.) 263

Gibt es eine orale Trichomonaden-Infektion? (M e y e r - R o h n , J.) 282

Wie ist die moderne Behandlung des Erysipels? (W e b e r , G.) 326

Kann nach dem Stand des heutigen Wissens Chininmercuribisulfat zur Prophylaxe bei Geschlechtskrankheiten empfohlen werden?

(B r e h m, G.) 378

Uberempfindlichkeit gegen Progesteron bei Prurigo mit prämenstrueller Exazerbation ( $\mathrm{H}$ o is ch e n, W., S t e ig le d e r, G. K.) 398

Schwere Thrombozytopenie mit hämorrhagischer Diathese bei infektiöser Mononukleose (B rit t in ger, G., H e n n e, G., K ö n i g , E., Ma s s on, M., St e in a c ker H. G.) 400

Die Behandlung der Dermatomykosen (F i s c h e r, E.) 411

Bourneville-Pringlesche Phakomatose mit Situs inversus, Doppelniere beiderseits und rezidivierendem Spontanpneumothorax $(\mathrm{O} \mathrm{d} \mathrm{y}$ R., B e r g e $r$, H.) 488

Therapie des Melanoms (Korting. G. W.) 501

Die Therapie des Ekzems (B u rkh a rd t, W.) 598

Ungelöste Probleme der Lepraforschung. Anmerkungen zu einer Leprologen-Tagung in Washington (A s s h a u e r, E.) 606
Therapie der Mykosen in der inneren Medizin (W e g m a n n, T.) 713

Antigenbindung durch zirkulierende Leukozyten bei der verzögerten lokalen Insulinallergie ( $\mathrm{F}$ e d e $\mathrm{r}$ li n, 'K., He in e ma nn, G., G i gli, I., Dits chuneit, H.) 814

Therapie des Erysipel (A d a m, W.) 821

Kosmetische Warzenbehandlung im Kaltkauterverfahren u. Ausübung der Heilkunde nach dem Heilpraktikergesetz (S c h mel c her, R.) 869

Stellung und Wert der Arsen-Behandlung aus dermatologischer Sicht (P e t z old t, D.) 909

Was leistet die Thormählensche Probe zur Früherkennung des $\mathrm{Me}$ lanoms? (K a l k of f, K. W.) 919

Zur Klinik, Pathogenese und Therapie des Herpes zoster, insbesondere zur Häufigkeit des Zoster duplex unilateralis ( $\mathrm{H}$ a u s e $\mathrm{r}, \mathrm{W}$.) 998

Penicillin-Allergie ( $d$ e W e c k, A. L.) 999

Therapie des Pemphigus ( $\mathrm{N}$ ö d l, F.) 1093

Welche Behandlung ist bei einem pigmentierten und behaarten Naevus eines dreijährigen Kindes heute angezeigt? (K o r t in g, G. W.) 1098

Wie entstehen umschriebene Lipodystrophien beim Diabetiker und auf welche Weise können sie chronische Schmerzzustände auslösen? ( $\mathrm{S} \mathrm{c} \mathrm{hr}$ a de r, A.) 1171

\section{Herz und Kreislau}

Die Regelung der Koronardurchblutung ( $\mathrm{H}$ i r c h e, Hy.) 1

Ergebnisse der plastischen Korrektur der Aortenstenose

(Z e n k e r, R., S e i d e l, W., Fin sterer, U., K linner, W.) 8

Zur Beurteilung der Q-Zacke im Elektrokardiogramm (Hilmer, W.) 15

Indikation zur Rekonstruktion der Beckenarterien bei kombinierten Gefäßverschlüssen ( $\mathrm{W}$ a i b e l, P.) 23

Maßnahmen bei akutem Kreislaufstillstand (H o s s li , G.) 29

Elektrische Defibrillation bei Vorhofflimmern? (E f f e r t , S.) 39

Kann man allein aus erhöhtem Blutcholesterin und Arcus senilis auf eine allgemeine Atherosklerose schließen? (S c h e t t l e r, G.) 43

Wann sind vasodilatierende Mittel bei Schocktherapie indiziert? (A hnefeld, F. W., Frey, R.) 43

Welche Erfolge hat die Behandlung von koronaren Durchblutungsstörungen mit Sexualhormonen?

(K le p z i g, H.) 44

Grundlagen zur Behandlung von Venenerkrankungen der Extremitäten mit Kompressionsverbänden ( $\mathrm{H}$ a i d, H.) 73

Kann sich eine postenzephalitische Hypertonie noch nach jahrzehntelangem Intervall manifestieren? (S t u r m , A.) 94
Ist beim anaphylaktischen Schock die Anwendung von Corticosteroiden neben der sonstigen Schocktherapie indiziert? (W e r n e r, M.) 95

Therapie der Herz- und Lungenveränderungen bei Wirbelsäulendeformierung? (H e y m e r, A.) 138

Ist Streckentauchen oder Tieftauchen gesundheitsschädlich? (Hollman n, W.) 187

Einfluß des sympathiko-adrenalen Systems auf Herz-Kreislauf-Regulation und unveresterte Fettsäuren des Blutserums (K rzy wa n e k H. J., R a a b, W., B ö hl e, E.) 193

Vergleichende Untersuchungen über die blutdrucksenkende Wirkung von $\alpha$-Methyl-Dopa und $\alpha$-Methylm-thyrosin (Holt m eier, H. J., v. Klein-Wisenberg, A. Marongiu, F.) 198

Rezidivmitralstenosen (I $\mathrm{r} \mathrm{mer}, \mathrm{W}$. Obla de n, H., Delf ino, A. J.) 433

Behindert die Varizen-Sklerotherapie eine spätere Operation? (S i g g , K.) 467

Harnkonzentrierung bei essentieller Hypertonie (M e r t z , D. P.) 529

Zur thrombolytischen Therapie des frischen Herzinfarkts. I. Einführung Behandlungspläne, allgemeine klinische Ergebnisse

(Schmutzler, R., Heckner, F., Körtge, P., van de Loo, J., Pezold, F. A., Poli wod a, H., Praetorius, F., Zekorn, D.) 581

Zur Klinik metastatischer Herzgeschwülste (B r a c h, F.) 593

Was ist über die Synchronisation vegetativer Funktionen mit rhythmisch einwirkenden Reizen bekannt? (A s c h of f, J.) 610

Morphologie, operative Behandlung und deren Ergebnisse bei 139 Sinus-venosus-Defekten (D e r $r$ a E., Irmer, W., T a rbiat, S.) 627

Die chirurgische Behandlung der Angina pectoris (S e nning, A.) 641

Die operative Prophylaxe der zerebralen Mangeldurchblutung (D o r n d orf, W.) 662

Sind diätetische Maßnahmen be Herzinfarkt heute noch nützlich? (S c h e t t l e r, G.), 703

Ist eine Nicotinsäuretherapie peripherer Durchblutungsstörungen vertretbar, wenn gleichzeitig stenokardische Beschwerden bestehen? (K l e pzi g , H.) 725

Wie erklärt man sich die drohende Niereninsuffizienz schwer Herzkranker bei $\mathrm{zu}$ brüsker Ausschwemmung? ( $\mathrm{S} \mathrm{ch}$ ir meis ter, J.) 726

Die Bedeutung abnormer Amplituden der Kammeranfangsgruppe im Elektrokardiogramm (H e in e c k e r, R.) 768

Welche diagnostische Aussagen erlauben Oszillo- und Rheographie bei peripheren Gefäßprozessen? (S c h o o p, W.) 784

Alterschirurgische Probleme bei arteriellen Verschlußkrankheiten (L o o s e , K. E.) 812 
Zur Beurteilung der Q-Zacke im Elektrokardiogramm. Bemerkungen zur gleichnamigen Veröffentlichung $v$. W. Hilmer in dieser Wochenschrift 91 (1966), 15 ( $\mathrm{He}$ in ri c h, F.) 859

Laboratoriumsuntersuchungen bei Arteriosklerose (W a g e n e r, $\mathrm{H}$.) 861

Der chronische Verschluß von Gliedmaßenarterien. Durchblutungsförderung (W i d m e r, L. K.) 863

Welche Bedeutung haben Extrasystolen unter Belastung nach anscheinend völlig ausgeheilter Myocarditis nach Wiedererlangung der vollen Arbeitsfähigkeit? (H o l z m a n n, M.) 872

Bestehen Erfahrungen in der Anwendung von Corticosteroiden bei Höhenkrankheit beim Bergsteigen? (S c h w a l b , H.) 873

Vitamin-D-hypercalcämische Herzu. Gefäßerkrankungen ( $\mathrm{B}$ e u r e n, A. J., A pitz, J., S to e rmer, J., $\mathrm{Schlange,} \mathrm{H} ., \mathrm{Ka}$ iser, B., v. B erg, W., Jörgense n, G.) 88

Stufenbelastung zur Beurteilung der körperlichen Leistungsfähigkeit u. der Koronarreserve (Ka l t e n b a c h, M.) 884

Angiographie bei Beckenarterienverschlüssen zur Indikationsstellung für rekonstruktive Gefäßoperationen (Berghaus, H., Kauf m a n $n, H$.) 894

Zur thrombolytischen Therapie des frischen Herzinfarktes. II. Ergebnisse der elektrokardiographischen Untersuchungen (P o li w o d a, H., Di ed erich, K. W., Schnei der, B., R od e nburg, R., Heckner, F., Körtge, P., van de Loo, J., Pezold, F. A., Praetorius, F., Schmutzler, R. Z e k orn, D.) 978

Postmyokardinfarkt-Syndrom als tödliche Komplikation (B o c k e l, P., D o y r a n, E.) 1040

Differenzierung verschiedener Formen des Schocks durch einfache Meßverfahren (L u t z , H.) 1043

Reninaktivität im Plasma bei normalem, erhöhtem und erniedrigtem Blutdruck (Heintz, R., Lotz, W.) 1074

Zur Bedeutung des regeltheoretischkybernetischen Konzepts in der Medizin (D ris c h e l, H.) 1164

Gibt es Aquivalente eines Gichtanfalles, die eine Angina pectoris vortäuschen und im Elektrokardiogramm flüchtige Symptome eines Infarktes zeigen? (Z ölln e r, N.) 1171

Behandlung des Lungenödems mit Procain? (B u d e l m a n n, G.) 1202

\section{Infektionskrankheiten, Immunität}

Respiratory-syncytial-Virus-Infektionen im Kindesalter. Virologische Untersuchungen ( $\mathrm{S} \mathrm{ch}$ h e w e is, K. E., Käckell, M. Y., Nolt e, R., S in a pius, D., B r a n d is, Die thymoiden Organe des Immunitätssystems ((G ü $n t h$ e $r, O)$.
Zur Schließung von Lebensmittelbetrieben bei Infektionskrankheiten ( T r ü b, C. L. P.) 133

Wo ist in Europa mit dem Auftreten von Amöbenruhr zu rechnen? (F i s c h e r, L.) 138

Respiratory-syncytial-Virus-Infektionen im Kindesalter. Klinik und pathologische Anatomie (S c h n e we is, K.E., Käckell, M.Y., Nolte, R., Sinapius, D. B randis, H.) 153

Die Behandlung des Leberkomas (Martin i, G. A.) 221

Gibt es gesicherte Fälle von Mononucleosis infectiosa mit konstant negativer Reaktion nach Hanganutziu-Deicher? (Vorlaender, K. O.) 233

Eine Facialisparese durch Adenovirus-Typ-5-Infektion

( Li e bscher, S., Wunderli c h, P.) 256

Zur Klinik, Pathogenese und Therapie des Herpes zoster, insbesondere zur Häufigkeit des Zoster duplex unilateralis ( $\mathrm{Hell}$ e, S.) 263

Gibt es unspezifische Erhöhungen des Antistreptolysintiters? (S ü d h of , H.) 282

Häufigkeit und Behandlung der Mumps-Enzephalitis? (V i vell, O.) 282

Intravenöse Grippetherapie mit 0,01 g Cyanquecksilber? (L e n d l e, L.) 325

Wie ist die moderne Behandlung des Erysipels? (W e b e r , G.) 326

Ursachen der Leptospirosen in Nordafrika ( $\mathrm{Ma}$ ill o u $\mathrm{x}, \mathrm{M}$.) 370

Untersuchungen über das Vorkommen von Virämie nach oraler Poliomyelitisimpfung mit Sabin Typ 1 . Stamm LSc 2 ab (H a a S, R., E s s e r, I., S c h u m a ch er, H., M a jer, M.) 385

Schwere Thrombozytopenie mit hämorrhagischer Diathese bei infektiöser Mononukleose (Brittinge r, G., H e nn e, G.; K ö n i g, E., Masson, M., Stein a cker, H. G.) 400

Erkrankungen des Respirationstraktes durch Mycoplasma pneumoniae (W itzle b. W., S pröss i g, M. Anger, G. Heidle r, S.) 429

Die Therapie der Pocken (Stickl, H.) 455

Ist eine Wohnungsentseuchung bei Hepatitis epidemica sinnvoll? (R o e $\mathrm{m}$ e r, G. B.) 466

Zur Notwendigkeit der BCG-Schutzimpfung. Beobachtungen bei umschriebenen Häufungen von Tuberkulose (Truckenbrod t, $\mathrm{H}$. $\mathrm{Hirs} \mathrm{chmann,} \mathrm{E.)} 479$

Virologische und klinische Beobachtungen nach aktiver Masernschutzimpfung. 2. Mitteilung ( $\mathrm{H}$ a a s, R., $\mathrm{Vivell}, \mathrm{O}$., Buentello-Malo, L., R e is s, G., B e rn ing, D.) 569

Erste Ergebnisse mit einem Fünffachimpfstoff. gegen Masern, Diphtherie, Pertussis, Tetanus, Poliomyelitis (Enders-Ruckle, G., S pies s, H. W o lf, H.) 575

Ungelöste Probleme der Lepraforschung. Anmerkungen zu einer Leprologen-Tagung in Washington (A s sha uer, E.) 606
Diagnose der Lungenmykose (W e g $\mathrm{m}$ a n n, T.) 711

Therapie der Mykosen in der inneren Medizin (W e g m a n n, T.) 713

Die heutige epidemiologische Bedeutung und Problematik der Salmonella-Erkrankungen ( $G$ ä $r$ tn e r , H.) 803

Therapie des Erysipel (A d a m, W.) 821

Zum Problem der Infektion des Menschen, insbesondere des Kindes, mit Larven von Tier-Askariden (S c h o o p, G., L a m in a, J.) 848

Beitrag zur Pathogenese der Insulinresistenz ( $D$ it s chune it, H., Fe d e r l in, K.) 853

Salmonellenmeningitis beim Erwachsenen (Stille, W., Schoop, H. D.) 891

Sind Quecksilberschmierkuren zur Prophylaxe der Rubeolenembryopathie zu empfehlen? (Ma yer, H.) 919

Sind für eine Tetanus-Grundimmunisierung zwei oder drei Impfungen mit einem Aktiv-Impfstoff zu empfehlen? (R o e m e r, G. B.) 966

Zur Klinik, Pathogenese und Therapie des Herpes zoster, insbesondere zur Häufigkeit des Zoster duplex unilateralis ( $\mathrm{H}$ a u se $\mathrm{r}, \mathrm{W}$.) 998

Menschliche Malaria: eine Zoonose? (A s s h a u e r, E.) 1003

Untersuchungen über Häufigkeit und Bedeutung von Infektionen mit Mycoplasma pneumoniae

(K r e ch, U., Modd e, H.) 1013

Uber die Anwendung von Oxacillin in hohen Dosen und von Amphotericin $B$ bei akutem Nierenversagen ( $\mathrm{S} \mathrm{chr}$ öd er, E., O h m, H. G., D e u p m a n n, F. J.) 1035

Wirksamkeit und Risiko der "Grippe-" impfungen"? (M a a s s , G.) 1098

Immundepressive Wirkung von Thalidomid? (L e $\mathrm{nz}$, W.) 1132

Wann und aus welchem Anlaß wurde die erste Quarantäne eingerichtet? (R a t h, G.) 1134

Infektion mit dem Virus der Zentraleuropäischen Enzephalitis in Südwestdeutschland (A c ker m a n n, R., S c h eid, W., K ü p p e r, B.) 1141

Bemerkungen zur Frage der Indikationen und der sogenannten Entgiftung des Streptomycins (Dihydrostreptomycin) (K u schinsky, G.) 1150

Zur Behandlung bakterieller Infektionen mit Kombinationspräparaten von Streptomycin bzw. Dihydrostreptomycin und Penicillin ( $\mathrm{N}$ a u m a n n, P.) 1152

Pleurodynie und akutes abdominelles Syndrom (A re nd, P.; M a r t in i, G. A.) 1180

Eine ungewöhnlich leichte Herpessimplex-Erkrankung bei einem Neugeborenen ( $\mathrm{K}$ ünz er, W., Majer, M., Denz, Ch.) 1183

\section{Innere Sekretion, Vitamine}

Welche Erfolge hat die Behandlung von koronaren Durchblutungsstörungen mit Sexualhormonen? (K le p z i g, H.) 44 
Ist die Blutzuckerbestimmungsmethode nach Crecelius-Seifert in der Praxis brauchbar und zuverlässig? (Mehnert, H., Förster, H.) 95

Die Registrierung des Achillessehnenreflexes als diagnostisches Hilfsmittel bei Schilddrüsenerkrankungen (Em rich, D., Maron g i u, F.) 101

Untersuchungen zur Wirkung der Ascorbinsäure auf den Kohlenhydratstoffwechsel ( $\mathrm{Meh}$ e e r t, H., För s ter, H., Fun ke, U.) 106

Behandlung des Cushing-Syndroms (Hen i, F.) 174

Zum Wirkungsmechanismus der Steroidhormone

(D rew s, J., Wa cker, A.) 178

Kindlicher Diabetes mellitus infolge fetaler Inselschädigung? Zur Hypothese von Cornelia van Beek (Schreibenreiter, S., Thal$\mathrm{h}$ a $\mathrm{m}$ m e $r, 0)$.

Uber ein Lipom der Nebenniere mit Phäochromozytomsymptomatik (L a n g e, H. P.) 254

Zur Therapie der Retinopathia diabetica mit Fructose ( $\mathrm{S} \mathrm{chrader}$, K. E., Mähr, G., Neumann, P.) 306

Die Insulinresistenz ( $\mathrm{P}$ f e i f f e r, E F.) 314

Durchführung und Aussage des Tol-

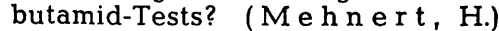
326

Wie ist das Auftreten eines Diabetes mellitus bei Leberzirrhose $\mathrm{zu}$ erklären? (Cre u t z f e ld, W.) 377

Zur postnatal erworbenen Toxoplasmose bei Kleinkindern ( $\mathrm{P}$ a u l, J.) 389

Uber Häufigkeit und klinische Bedeutung von Autoantikörpern bei Schilddrüsenerkrankungen, insbesondere bei der Hyperthyreose (Uthgenannt, H., Müller, W., We in re i c h, J.) 437

Laboratoriumsbefunde bei Anorexia nervosa ( $\mathrm{F} \mathrm{r} \mathrm{a} \mathrm{h} \mathrm{m,} \mathrm{H.)} 499$

Thyreotoxische Myopathie (Kluthe, R., Mertz, D.P., Wesle, H., Veih elm a n n, D.) 536

Der ${ }^{131} \mathrm{~J}$-Trijodthyronintest und seine Kombination mit der mikrochemischen PBJ-Bestimmung im endemischen Kropfgebiet Südbadens (Meigen, B., Mertz, D. P.) 541

Anabole Hormone und Stimmstörungen (S c h l ö n d o r f f , G.) 555

Zur Kenntnis eines seltenen, durch ein Pankreasadenom verursachten Krankheitssyndroms ( $\mathrm{Z}$ e n k e r R., F ore ll, M. M., Erpenbeck, R.) 634

Der labile Diabetes. Pathophysiologie, Klinik, Therapie (P e t ri d e s , P.) 689

Die Begutachtung des Diabetes mellitus ( $\mathrm{S} \mathrm{ch}$ ö f f l in $\mathrm{g}, \mathrm{K}$.) 694

Kann Dauertherapie mit 16-24 mg Methylprednisolon täglich bei $\mathrm{M}$. Addison ein Ulcus duodeni verursachen? (W e i s b e c k e r, L.) 725

Aktuelle Diabetesprobleme in Klinik und Praxis ( $\mathrm{Me} \mathrm{h} \mathrm{n}$ e $\mathrm{t}, \mathrm{H}$.) 744

Latent diabetische Stoffwechsellage bei Eltern juveniler Diabetiker (Brauns teiner, H., Hansen, W., Jung, A., S a il e r, S.) 750
Vergleich klinischer Methoden zur Erfassung des latenten Diabetes mellitus

(S c riba, P. C., S chwarz, K. $\mathrm{H}$ of $\mathrm{m}$ a n n, G. G.) 735

Die endokrine Beeinflussung des Stoffwechsels im Fettgewebe ( $L$ ö f f l e r, G., W e inges, K. F.) 773

Antigenbindung durch zirkulierende Leukozyten bei der verzögerten lokalen Insulinallergie $(\mathrm{F}$ e d e r li $\mathrm{n}$, K., H e in e m a n n, G., G i gli, I., Dit s c huneit, H.) 814

Paraneoplastischer Hypercorticismus. Klinische, biochemische und morphologische Befunde $(\mathrm{H}$ änz e, S. Pi er a c h, C. A.) 837

Beitrag zur Pathogenese der Insulinresistenz ( $D$ it s chuneit, $H$. Fed e rli n, K.) 853

Werden die einzelnen Biguanid-Präparate unterschiedlich vertragen? (M e hn e r t , H.) 873

Vitamin-D-hypercalcämische Herz- u. Gefäßerkrankungen (B e u r e n, A. J., A pitz, J., Sto ermer, J., $\mathrm{Schlange,H}, \mathrm{K}$ a iser, B. v. B erg, W., Jörgensen, G.) 881

Synacthen-Kurztest zur Beurteilung der Nebennierenrindenfunktion ( O t to, H., Minneker, C. $\mathrm{S}$ p a e th e, R.) 934

Uber die Wirkung eines neuen Depot-Biguanids auf den Kohlenhydrat- und Eiweißstoffwechsel bei Diabetes mellitus ( $M$ ü t in $\mathrm{g}, \mathrm{D}$.) 939

Die Diagnostik der primären und sekundären Nebennierenrindeninsuffizienz ( $\mathrm{T}$ a $\mathrm{m} \mathrm{m}$, J.) 957

Klinik der Insulinresistenz (D a w e $\mathrm{k}$ e r, H.) 973

Kann versehentliche intravenöse Injektion von Citrat bei der Blutentnahme zur Blutsenkungsreaktion nach fünf Stunden einen tetanischen Anfall auslösen ( $\mathrm{K}$ u s c h i n s k y , G.) 1004

Wie behandelt man eine Mastodynie? (D ö r i n g , G. K.) 1005

Wie kann man übermäßiges Längenwachstum bei Jugendlichen beeinflussen? (V o g t , D.) 1005

Besondere klinische Formen des Thymus-Syndroms ( $\mathrm{B}$ ü hn e r, M. G e h rma n n, G.) 1105

Die klinischen Symptome der Hyperthyreose und ihre Bedeutung für die Diagnostik (G ünther, R., L a u b i n g e r, G.) 1144

Zur Bedeutung des regeltheoretischhybernetischen Konzepts in der Medizin (D r is c h e l, H.) 1164

Wie entstehen umschriebene Lipodystrophien beim Diabetiker und auf welche Weise können sie chronische Schmerzzustände auslösen? (S c h r a der, A.) 117

Kann eine Hypoglykämie ein Halbseitensyndrom mit bleibenden neurologischen Schäden auslösen? (E y e r, H.) 1202

Ursache des Jodmangels in manchen Gegenden der Alpenländer (E y e r, H.) 1202

\section{Kinderheilkunde}

Respiratory-syncytial-Virus-Infektionen im Kindesalter. Virologische Untersuchungen (Schneweis, K. E., Käckell, M. Y., Nolte, R., Sinapius, D., Brandis, H.) 53

Nierenvenenthrombose beim Neugeborenen. Ein kasuistischer Beitrag. (T e i x i d or, J., He lb i g, D.) 57

Therapeutische Möglichkeiten bei progressiver Muskeldystrophie (B e c k m a n n, R., von O s t e n, V.) 108

Methodische Hinweise zur Diagnostik von Chromosomen-Anomalien (S t o e ckenius, M.) 125

Auswirkungen transplazentarer Blutzellenpassagen auf das Kind (O e h m e , J.) 129

Respiratory-syncytial-Virus-Infektionen im Kindesalter. Klinik und pathologische Anatomie (S chn eweis, K. E., Käckell, M. Y., Nolte, R., Sinapius, D., $\mathrm{Brandis}, \mathrm{H}$.) 153

Zur Frühest- und Frühdiagnose der sogenannten angeborenen Hüftgelenksluxation (D ö r r, W. M.) 168

Kindlicher Diabetes mellitus infolge fetaler Inselschädigung? Zur Hypothese von Cornelia van Beek (Schreibenreiter, S., Thalh a m m e r, O.) 216

Die klinischen Symptome der Hyperthyreose und ihre Bedeutung für die Diagnostik (G ün th er, R., L a u b ing e r, G.) 1144

Zur Bedeutung des regeltheoretischkybernetischen Konzepts in der Medizin (D ri s c h e l, H.) 1164

Wie entstehen umschriebene Lipodystrophien beim Diabetiker und auf welche Weise können sie chronische Schmerzzustände auslösen? ( $\mathrm{S} \mathrm{c} \mathrm{h} \mathrm{r} \mathrm{a} \mathrm{d} \mathrm{e} \mathrm{r,} \mathrm{A.)} 1171$

Kann eine Hypoglykämie ein Halbseitensyndrom mit bleibenden neurologischen Schäden auslösen? (E y e r, H.) 1202

Ursache des Jodmangels in manchen Gegenden der Alpenländer? (E y e r, H.) 1202

Was dürfen wir vom Elektroenzephalogramm erwarten? (S c h a r f e t t e r, Ch.) 223

Amniozentese u. intrauterine Transfusion: neue Wege der Behandlung der Neugeborenenerythroblastose (S a c h t l e b e n, P.) 229

Hochdosierte intermittierende Methotrexat-Therapie bei akuten Leukosen im Kindesalter (B l ä k e r, F., L a n d b e c k , G.) 245

Eine Facialisparese durch Adenovirus-Typ-5-Infektion

(L i e b s cher, S., Wunderlich, P.) 256

Zur pathologischen Anatomie der Pneumonieformen in der Perinatalperiode (W ö c k e l, W.) 273

Häufigkeit und Behandlung der Mumps-Enzephalitis (V i v e 1 1, O.) 282

Die Kardiafunktion im Verlaufe der Kindheit (D i t t r i c h, J. K.) 303 
Uber das infantile nephrotische Syndrom bei kongenitaler Glomerulonephritis (Ros se n b e ck, H. G. Margraf, O., Hof mann, D.) 348

Zur kausalen Pathogenese des infantilen nephrotischen Syndroms bei kongenitaler Glomerulonephritis (R o s s e n b e c k, H. G.) 363

Untersuchungen über das Vorkommen von Virämie nach oraler Poliomyelitisimpfung mit Sabin Typ 1 . Stamm LSc 2 ab (H a a s, R., Es ser, I., S chum a cher, H., Maj e r, M.) 385

Zur postnatal erworbenen Toxoplasmose bei Kleinkindern ( $\mathrm{Pa} \mathrm{u} \mathrm{l,} \mathrm{J.)}$ 389

Myasthenia gravis bei eineiigen Zwillingen (A d l e r, E.) 396

Glykogenose Typ I (v. Gierke) früher mitgeteilt als familiäre Hyperlipidämie (Fuhrmann, W., J a c ov C i c, S., Y i Y ung H s i a, D.) 453

Die plazentare Transfusion

(S c h e n c k, W.) 458

Uber die Diagnostik und Therapie der erblichen Lese-RechtschreibeSchwäche (W e in schenk， C.) 461

Kindesbeschneidung und Anscheinsbeweis bei behauptetem ärztlichem Kunstfehler (S c h m el ch e r, R.) 464

Ist eine Wohnungsentseuchung bei Hepatitis epidemica sinnvoll? (R o e m e r, G. B.) 466

Ist die Credésche Augenprophylaxe bei jeder Geburt gesetzlich vorgeschrieben? (K ö n i g , P. A.) 467

Zur Notwendigkeit der BCG-Schutzimpfung. Beobachtungen bei umschriebenen Häufungen von Tuberkulose (Truckenbrodt, $\mathrm{H}$. H i r c h m a n n, E.) 479

Laboratoriumsbefunde bei Anorexia nervosa (F r a h m, H.) 499

Virologische und klinische Beobachtungen nach aktiver Masernschutzimpfung. 2. Mitteilung $(\mathrm{H}$ a a s, $\mathrm{R}$. Vivell, O., Buentello-Malo, L., R e is s, G., B e rning, D.) 569

Erste Ergebnisse mit einem Fünffachimpfstoff gegen Masern, Diphtherie, Pertussis, Tetanus, Poliomyelitis

(Enders-Ruckle, G., Spiess, H., W ol f, H.) 575

Mechanismus, Zeitpunkt und Häufigkeit der Entwicklung einer Rh-Inkompatibilität? (M a r tiu s, G.) 610

Morphologie, operative Behandlung und deren Ergebnisse bei 139 Sinus-venosus-Defekten ( D e r r a , E., I r m e r, W., T a r b i a t, S.) 627

Welche serologischen Tests sind zur sicheren, rechtzeitigen Erkennung von Blutgruppenunverträglichkeiten zwischen Mutter und Kind erforderlich? (Spie lmann, W.) 666

Latent diabetische Stoffwechsellage bei Eltern juveniler Diabetiker (B r a unst einer, H., Hansen, W., Jung, A., Sailer, S.) 750
Soll bei Linkshändern in der Schule der Versuch einer Umstellung auf Rechtshändigkeit durchgeführt werden? ( $\mathrm{H} \mathrm{e} \mathrm{l} \mathrm{l} \mathrm{b} \mathrm{r} \mathrm{ü} \mathrm{g} \mathrm{g} \mathrm{e} \mathrm{,} \mathrm{T} \mathrm{h.)} 783$

Zur Problematik der perinatalen Blutungsübel (K ü n $\mathrm{z}$ e r, W.) 793

Myelomeningocele und Hydrozephalus beim Säugling ( $\mathrm{Kuhlendahl}$ H.) 801

Hyalin-Membran-Syndrom ( $\mathrm{Hirs} \mathrm{ch-}$ $\mathrm{m}$ a $\mathrm{n} \mathrm{n}, \mathrm{E}$.) 822

Zum Problem der Infektion des Menschen, insbesondere des Kindes, mit Larven von Tier-Askariden (S c h o o p, G., L a m i n a, J.) 848

Vitamin-D-hypercalcämische Herz- u. Gefäßerkrankungen (B e u r e n, A. J., A pitz, J., Stoermer, J.,

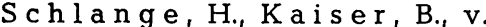
B erg，W., Jörgensen, G.) 881

Sind Quecksilberschmierkuren zur Prophylaxe der Rubeolenembryopathie zu empfehlen? (Mayer , H.) 919

Zur Diagnostik und Therapie von Harnabflußstörungen im Kindesalter (Hoh e nf elln er, R., Sö$\mathrm{kel}$ a nd, J., S t ra u b, E.) 930

Sind für eine Tetanus-Grundimmunisierung zwei oder drei Impfungen mit einem Aktiv-Impfstoff zu empfehlen? (R o e $\mathrm{m} \mathrm{e} \mathrm{r}, \mathrm{G}$. B.) 966

Wie behandelt man eine Mastodynie? (D ö r i n g, G. K.) 1005

Wie kann man übermäßiges Längenwachstum bei Jugendlichen beeinflussen? (V o g t , D.) 1005

Klinische und immunologische Aspekte des Morbus Werlhof (G e h r m a n n, G.) 1069

Welche Behandlung ist bei einem pigmentierten und behaarten $\mathrm{Nae}$ vus eines dreijährigen Kindes heute angezeigt? (Kortin g, G. W.) 1098

Immundepressive Wirkung von Thalidomid? (L e $\mathrm{nz}, \mathrm{W}$.) 1132

Eisenmangelanämie im Kindesalter (K le i hauer, E.) 1162

Muß bei einem Moro-positiv gewordenen Säugling eine Isoniazid-Behandlung durchgeführt werden auch wenn dieser normal gedeiht? (S p i e s s, H.) 1171

Eine ungewöhnlich leichte Herpessimplex-Erkrankung bei einem Neugeborenen (K ü nzer, W. Majer, M., Denz, Ch.) 1183

Welche Diät ist bei akuter Glomerulonephritis im Kindesalter zu empfehlen? (D i t t r i c h) 1202

\section{Mund, Hals, Nase, Ohren}

Ist die sogenannte essentielle Trigeminusneuralgie ein "rein funktionelles Leiden"? (S e if e r t, K.) 321

Die Telecaesiumbehandlung der Larynx- und Hypopharynxkarzinome (K a u f m a n $n, H$.) 339

Die Diagnose des Morbus Menière (P f a l z, R.) 409

Klinik, Therapie und Morphologie der Tumoren der nicht chromaffinen Paraganglien ( $\mathrm{Hel} \mathrm{pap}$, E., H e l p a p, B.) 493
Anabole Hormone und Stimmstörungen (S c h lö nd orf f, G.) 555

Innenohrschwerhörigkeit bei Dystrophia myotonika u. Dystrophia muscularis progressiva ( $\mathrm{K} \mathrm{u} \mathrm{h} n, E$., E y, W.) 947

Darf der Arzt während einer Mandeloperation dem unruhigen Patienten Schläge versetzen? ( $\mathrm{S} \mathrm{chm} \mathrm{e} \mathrm{l-}$ cher, R., Kohlh a a s, M.) 963

Worin besteht derzeit die erfolgversprechendste Therapie einer stark ausgeprägten Paradontose bei sonst gut erhaltenem Gebiß? (W a n n n en $\mathrm{m} \mathrm{a} \mathrm{ch} \mathrm{e} \mathrm{r,} \mathrm{E.)} 967$

Hals-Nasen-Ohren-ärztliche Erfahrungen mit der operativen Kälteanwendung ( $\mathrm{W}$ ullstein, $H$., Gerlach, J., Spuler, H. Z e h $\mathrm{m}, \mathrm{S}$.) 1016

Bemerkungen zur Frage der Indikationen und der sogenannten Entgiftung des Streptomycins (Dihydrostreptomycin) (Ku schinsky G.) 1150

Bemerkungen zur Frage der Penicillin-Streptomycin-Kombinationen aus hals-nasen-ohren-ärztlicher Sicht (D e c h e r, H.) 1158

\section{Nervensysteme und Psychiatrie}

Uber die Neuroanatomie in der $\mathrm{Me}$ dizin von heute (F le is ch ha u r K.) 75

Kann sich eine postenzephalitische Hypertonie noch nach jahrzehntelangem Intervall manifestieren? (S t u r m , A.) 94

Die medikamentöse Therapie der Epilepsie ( $\mathrm{H} \mathrm{e} \mathrm{s} \mathrm{s}, \mathrm{R}$ ) 126

Neuere Methoden in der Diagnostik spinaler Angiome (P i a, H. W. Vo ge Is a n g, H.) 173

EinfluB des sympathiko-adrenalen Systems auf Herz-Kreislauf-Regulation und unveresterte Fettsäuren des Blutserums (K r z y w a nek, H. J., Ra a b, W., B öhl e, E.) 193

Was dürfen wir vom Elektroenzephalogramm erwarten? (Sc harfet $\mathrm{t}$ e r, Ch.) 223

Eine Facialisparese durch Adenovirus-Typ-5-Infektion

(L i e b s ch er, S., Wunderlich, P.) 256

Kniegelenks- und Hüftgelenkskontrakturen bei narbiger Irritation des sensiblen Astes des Nervus obturatorius (F e t t w e is, E.) 313

Die Lokalisation von biogenen Aminen im Zentralnervensystem (F l e is ch h a u e r, K.) 318

Ist die sogenannte essentielle Trigeminusneuralgie ein "rein funktionelles Leiden"? (S e if e r $t, K$.) 321

Ist die Einwilligung geistig beeinträchtigter Personen in Heileingriffe möglich oder ersetzbar? (Ko h 1 h a a s, M.) 323

Therapie nächtlicher Armschmerzen (Z i n k e, R.) 327

Myasthenia gravis bei eineiigen Zwillingen (A d l e r, E.) 396

Die Diagnose des Morbus Menière (P f a l z, R.) 409 
Umfang der Sicherungsvorkehrungen in einem psychiatrischen Krankenhaus (S c h m e l c h e r, R.) 416

Psychoanalytische Therapie endogener Depressionen? (K i e l holz, P.) 421

Wie sind die Erfolgsaussichten der Evers-Diät bei multipler Sklerose? (W e l t e, E.) 421

Das Multifidus-Dreieck-Syndrom (S c h m it t, A., Ki en l e, G.) 444

Hirntumor-Nachweise mit Radioisotopen (S c h n e i d e r, C.) 454

Zur Frage der Magenspülung bei Vergifteten (B a l z e reit, F., A r n o l d, W.) 485

Bourneville-Pringlesche Phakomatose mit Situs inversus, Doppelniere beiderseits und rezidivierendem Spontanpneumothorax (O d y , R., B e r g e $r, H$.) 488

Klinik, Therapie und Morphologie der Tumoren der nicht chromaffinen Paraganglien ( $\mathrm{Hel} \mathrm{p} \mathrm{a} \mathrm{p,} \mathrm{E.,}$ $\mathrm{H}$ e $\mathrm{l}$ p a p, B.) 493

Uber die diagnostische Bedeutung des Elektroenzephalogramms bei offenen Augen. Vorläufige Mitteilung ( $\mathrm{L}$ a n d o l t , H., L o r g é, M., $\mathrm{S} \mathrm{ch} \mathrm{m} \mathrm{id,} \mathrm{A.)} 539$

Die medikamentöse Behandlung des Parkinson-Syndroms ( $\mathrm{K}$ a e s e r, H. E.) 550

Das Schleudertrauma der Halswirbelsäule und seine verschiedene Folgen, Differentialdiagnose und Therapie (M üll e r, E.) 588

Die operative Prophylaxe der zerebralen Mangeldurchblutung (D orndorf, W.) 662

Welche Bedeutung hat das GammaSystem für die reflektorischen muskulären Verspannungen? (E r b s lö h, F.) 666

Enzephalopathie bei Blutkrankheiten (Bodechtel, G., Bor chers, H. -G., Kollmannsberger, A.) 673

Uber das cholinergisch-toxische Psychosyndrom prostigminbehandelter Myastheniker (A b e l, M., K o h l m e y e r, K.) 699

Veränderungen der AchillessehnenReflexzeit bei Patienten mit Niereninsuffizienz ( $\mathrm{F}$ an k ha u s e r, S., Morell, B., Re ubi, F.) 763

Konservative Therapie bei erhöhtem Hirndruck ( $\mathrm{H}$ e $\mathrm{m} \mathrm{m}$ e r, R.) 770

Myelomeningocele und Hydrozephalus beim Säugling ( $\mathrm{Kuhle} \mathrm{n} \mathrm{-}$ d a h l, H.) 801

Die Liquordynamik ( $\mathrm{H}$ e $\mathrm{m} \mathrm{m}$ e $\mathrm{r}, \mathrm{R}$.) 867

Salmonellen-Meningitis beim Erwachsenen (Stille, W., Schoop, H. D.) 891

Differentialdiagnose und operative Behandlung der spinalen Apoplexie (P i a , H. W.) 925

Innenohrschwerhörigkeit bei Dystrophia myotonica und Dystrophia muscularis progressiva ( $\mathrm{K} \mathrm{u} \mathrm{h} \mathrm{n,}$ E., E y, W.) 947

Erfahrungen mit sterotaktischen Eingriffen bei Epilepsie (O rthner, H., L o h m a n n, R.) 984

Zur Frage von Krankheitsdauer und Invalidität bei der multiplen Sklerose ( $\mathrm{M}$ üll e r, H. R.) 996
Beitrag zur Diagnostik der inneren Hirnvenenthrombose $(\mathrm{Fi}$ il i p a G., Reg li, F., Y a s a r g i l, M. G.) 1025

Zur Nosologie der innen Hirnvenenthrombose. Anatomie und pathologisch-anatomische Veränderungen (Filippa, G., Regli, F., Ya s a r g i l, M. G.) 1049

Was ist über gedächtnisfördernde $u$. -hemmende Substanzen bekannt? (v. B a u m g a r t e n, R.) 1060

Ursache und Therapie der Meralgia paraesthetica ( $\mathrm{Mum}$ enth a ler. M.) 1060

Differentialtherapeutische Erörterungen bei Rückenmarks- und Caudaverletzungen ( $\mathrm{L}$ a u s b e r g , G.) 1109

Die sekundären morphologischen Veränderungen des Gehirns nach Verletzung durch stumpfe Gewalt (M ülle r, N.) 1126

Quecksilberschmierkuren bei multipler Sklerose (S c h e i d) 1133

Infektion mit dem Virus der Zentraleuropäischen Enzephalitis in Südwestdeutschland (A c k e r m an n R., Scheid, W., Küpper, B. 1141

Heranziehung von Nervenärzten zum Notfallvertretungsdienst ( $\mathrm{S} \mathrm{c} \mathrm{h} \mathrm{m} \mathrm{e} \mathrm{l} \mathrm{c} \mathrm{h} \mathrm{e} \mathrm{r,} \mathrm{R.)} 1169$

Heranziehung von Fachärzten für Nervenheilkunde und Psychiatrie zum Notfalldienst (S chmelcher, R.) 1169

Zur Diagnostik und Therapie der epiduralen Blutung ( $\mathrm{P}$ ot h e, $\mathrm{H}$.) 1186

Diagnose der Polyneuropathien (J a n z e $n, R$.) 1192

Therapie bei Polyneuropathien (B a l z e re it, F.) 1194

Kann eine Hypoglykämie ein Halbseitensyndrom mit bleibenden neurologischen Schäden auslösen? (E y e r, H.) 1202

Pathologische Anatomie und Allgemeine Pathologie

Uber die Neuroanatomie in der $\mathrm{Me}$ dizin von heute (Fle is ch hau e r, K.) 75

Wie kann man im Saugbiopsiematerial die Tumorzellen von störenden Erythrozytenbeimengungen isolieren, ohne die phasenkontrastzytologische Beurteilbarkeit zu beeinträchtigen? (S e i f e r $\mathrm{t}, \mathrm{G}$.) 95

Uber die Häufigkeit des pathologischanatomischen Bildes der Schockniere und anderer Nierenerkrankungen im unausgewählten Obduktionsgut (Schubert, G. E. $\mathrm{K}$ ö b e rle, H.) 147

Fabry-Krankheit (Angiokeratoma corporis diffusum universale) Phosphatidspeicherkrankheit bei 2 Familien (D u ba ch, U. C., Gloor, F.) 241

Uber ein Lipom der Nebenniere mit Phäochromozytomsymptomatik (L a n g e, H. P.) 254
Zur pathologischen Anatomie der Pneumonieformen in der Perinatalperiode (W ö c k e l, W.) 273

Die Lokalisation von biogenen Aminen im Zentralnervensystem (F l e i s c h h a u e r, K.) 318

Uber das infantile nephrotische Syndrom bei kongenitaler Glomerulonephritis (R o s s e n b e c k, H. G. Margraf, O., Hofmann, D.) 348

Zur kausalen Pathogenese des infantilen nephrotischen Syndroms bei kongenitaler Glomerulonephritis (R o s s e n b e c k, H. G.) 363

Klinik, Therapie und Morphologie der Tumoren der nicht chromaffinen Paraganglien ( $\mathrm{Help}$ a, $\mathrm{E}$. $\mathrm{H}$ e l p a p, B.) 493

Glomerulonephritis. Aktuelle $\mathrm{Ge}$ sichtspunkte zur Pathogenese ( $\mathrm{R}$ ot h e r, Kl.) 551

Zur Nosologie der inneren Hirnvenenthrombose. Anatomie und pathologisch-anatomische Verändederungen (Fili p pa, G., Regli F., Y a s a r g i l, M. G.) 1049

\section{Arzt und Recht}

Melderecht oder Meldepflicht bei Fahruntüchtigkeit des Patienten (S c h m elch e r, R.) 41

Zur Frage der Schadensersatzpflicht des ärztlichen Fachgebiets wegen Uberschreitung des ärztlichen Fachgebiets ( $\mathrm{H}$ a f t e r, E.) 91

Zur Schließung von Lebensmittelbetrieben bei Infektionskrankheiten (T r ü b, C. L. P.) 133

Sind die Kosten eines während der Praxistätigkeit verschuldeten Verkehrsunfalles und dadurch entstehende Anwaltskosten steuerlich als Betriebsausgaben oder besondere Belastungen absetzbar, wenn das Verfahren wegen nicht möglicher Klärung des Unfallhergangs eingestellt wurde? (W u t h , K.) 139

Zum Begriff der "Ausbildung".. zum Facharzt in der Rentenversicherung angestellter Ärte $(\mathrm{H}$ a u e i s e $\mathrm{n}$, F.) 230

Nochmals: Zum Recht der Schwangerschaftsunterbrechung. Eine notwendige Ergänzung ( $\mathrm{K}$ o h l h a a s M.) 277

Ist die Einwilligung geistig beeinträchtigter Personen in Heileingriffe möglich oder ersetzbar? (K $\mathrm{K}$ hl $h$ a a s, M.) 323

Der Krankenhauschirurg ist gegenüber dem Krankenhausträger bei seiner gefahren- und schadensgeneigten Tätigkeit nicht regreßpflichtig (S c h m e l z e r, R.) 365

Kann der Amtsarzt im Wege der Dienstaufsicht die Entfernung einer Oberärztin veranlassen? (S c h m e lch e r, R.) 365

Dienstvergehen eines Amtsarztes (S c h m e l c h e r, R.) 366

Heranziehen von Fachärzten zum allgemeinen Notfallvertretungsdienst (S c h m e lc h e r, R.) 366

Der Bundesfinanzhof zur Feststellung einer typischen Berufskrankheit (W u t h, K.) 366 
Heranziehung von Nichtkassenärzten zum ärztlichen Notfallvertretungsdienst ( $\mathrm{S} \mathrm{c} \mathrm{h} \mathrm{m} \mathrm{e} \mathrm{l} \mathrm{c} \mathrm{h} \mathrm{e} \mathrm{r,} \mathrm{R.)} 416$

Umfang der Sicherungsvorkehrungen in einem psychiatrischen Krankenhaus (S c h m e l c h e r, R.) 416

Neue Nebentätigkeitsverordnung in Schleswig-Holstein

(S c h m e l c h e r, R.) 416

Neue Abgabenregelung für beamtete Chefärzte in Hamburg

(S c h m e lch e r, R.) 417

Neue Grundsätze zur Regelung der ärztlichen Notfallvertretung in Westfalen-Lippe

(S c h m elcher, R.) 417

Krankenhausarzt und Leichenschau (Ko h l h a a s, M.) 417

Beschränkung des Röntgenologen auf sein Fachgebiet

(S c h m elcher, R.) 463

Besoldung beamteter Arzte (S c h m el c h er, R.) 463

Trotz Zurücklassung eines Fremdkörpes:s im Operationsgebiet Ablehnung von Schmerzensgeld

(S c h m e l c h e r, R.) 463

Kindesbeschneidung u. Anscheinsbeweis bei behauptetem ärztlichem Kunstfehler (S c h melch e r, R.) 464

Neue wichtige Entscheidungen des Bundessozialgerichts $\mathrm{zu}$ kassenärztlichen Streitfragen $(\mathrm{H}$ a u e i s e $n, F$.) 465

Anwaltsk osten im sozialgerichtlichen Vorverfahren bei nachfolgendem sozialg erichtlichem Rechtsstreit grunds àtzlich erstattungsfähig (S c h m e l c h e r, R.) 509

Prüfung der Selbstkostenrechnung der Krankenanstalten durch Sozialversicherungsträger

(S c h m e l c h e r, R.) 509

Verfassungsmäßigkeit der Heilberufsgerichte (S chmelcher, R.) 509

Zum Begriff des Arzneimittels (S c h m e l c h e r, R.) 509

Beschränkung des Röntgenologen auf sein Fachgebiet auch im kassenärztlichen Bereich - EKG gehört nicht zum Fachgebiet des Röntgenologen (S c h melcher, R.) 513

Keine Arzthaftpflicht bei unterlassener Antitetanusinjektion ( $\mathrm{S} \mathrm{ch} \mathrm{m} \mathrm{e} \mathrm{l} \mathrm{c} \mathrm{h} \mathrm{e} \mathrm{r,} \mathrm{R.)} 558$

Kosmetische Eingriffe und Heilpraktikergesetz (S ch melcher, R.) 558

Sozialrichter für Kassenarztsachen (S c h m e lc h e r, R.) 558

Facharztanerkennung auf Grund Gerichtsurteils (S chmelcher, R.) 559

Arzneimittel und Apothekenzwang (S c h m e l c he r, R.) 608

Besondere Aufklärungspflicht bei Kropfoperationen

( $\mathrm{S} \mathrm{c} \mathrm{h} \mathrm{m} \mathrm{e} \mathrm{l} \mathrm{c} \mathrm{h} \mathrm{e} \mathrm{r,} \mathrm{R.)} 608$

Anästhesisten als Kassenärzte (S ch m elch e r, R.) 608

Inwieweit sind Honorare für ärztliche Prüfungen von Präparaten steuerbegünstigt? (Wu $\mathrm{t} h, \mathrm{~K}$.) 609

Stationäre Einweisung Unfallverletzter in ein nicht als Unfallkrankenhaus zugelassenes Krankenhaus (S c h m e l c h e r, R.) 664
Angestellte Chefärzte und freiberuf-

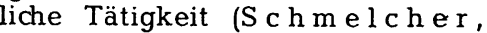
R.) 664

Arzthaftpflicht bei Narkoseschäden durch intraarterielle Injektion von Estil (S c h m e l c h e r, R.) 665

Die Begutachtung des Diabetes mellitus (S c h ö f f li n g, K.) 694

Sehschärfenbestimmung durch Optiker keine Ausübung der Heilkunde (S c h m e l c h er, R.) 720

Aufwendungen für Kongreßreisen als absetzbare Betriebsausgaben (S c h m e l c h e r, R.) 720

Ambulante Behandlung als Krankenhaus-Institutsleistung (S c h m e l c h e r, R.) 920

Honorierung ärztlicher Gutachten für Lebensversicherungsgesellschaften (S c h m elcher, R.) 721

Steuervergünstigungen für Honorare aus wissenschaftlichen Publikationen ( $W$ u t h, K.) 721

Kontrollen durch den Werksarzt bei erkrankten Werksangehörigen (M o r h a rd, K.) 722

Freiberufliche Tätigkeit und Abgabenpflicht beamteter Chefärzte (S ch m e lcher, R.) 780

Entschädigungsanspruch eines von einem gerichtlich bestellten Sachverständigen zugezogenen Facharztes (S c h m e l c h e r, R.) 780

Kassenärztliche Gemeinschaftspraxis (S c h m elcher, R.) 780

Neue Assistentenordnung in Rheinland-Pfalz ( $\mathrm{S} \mathrm{ch} \mathrm{mel} \mathrm{cher}, \mathrm{R}$.) 781

Neues Besoldungsgesetz in Hamburg (S c h m e lch e r, R.) 781

Medizinstudent, ärztliche Fortbildung und ärztliche Schweigepflicht (K o h lh a a s, M.) 781

Neues Gesetz über die Berufsgerichtsbarkeit in Hamburg (S c h m e lc h e r, R.) 826

Heranziehung von Nichtkassenärzten zum kassenärztlichen Notfallvertretungsdienst (S c h m e l che r, R.) 826

Nochmals: Darf der Arzt Klingel und Telefon abstellen? (Koh Ih a a s, M.) 826

Sonderparkerlaubnis für Ärzte in Niedersachsen (S ch melcher, R.) 869

Zusammenarbeit zwischen Arzt und Heilpraktikern (S ch melcher, R.) 869

Kosmetische Warzenbehandlung im Kaltkauterverfahren u. Ausübung der Heilkunde nach dem Heilpraktikergesetz (S ch melcher, R.) 869

Darf der Arzt während einer Mandeloperation dem unruhigen Patienten Schläge versetzen?

(S chmelcher, R., Kohlhaas, M.) 963

Folgen gedankenloser Weitergabe von Arztbefunden (v. B r ü c k e) 1056

Strahlenschäden durch Verletzung der ärztlichen Sorgepflicht und Grundsätze zum Schmerzensgeldanspruch (S ch m el ch e r, R.) 1058

Heranziehung von Nervenärzten zum Notfallvertretungsdienst (S c h m elcher, R.) 1169
Umsatzsteuerpflicht des Krankenhausröntgenologen im Durchgangsarztverfahren ( $\mathrm{Schmelcher}$, R.) 1169

Heranziehung von Fachärzten für Nervenheilkunde und Psychiatrie zum Notfalldienst (S ch melcher R.) 1169

\section{Strahlenkunde}

Möglichkeiten zur Bestimmung des Skelett-Calciumgehaltes in der Klinik (K rok ow ski, E.) 80

Makroradiographie mit Radiophosphor in der Diagnostik des Magenkarzinoms ( $\mathrm{H}$ a f t e $\mathrm{r}, \mathrm{E}$.) 90

Die Registrierung des Achillessehnenreflexes als diagnostisches Hilfsmittel bei Schilddrüsenerkrankungen (Em rich, D., Marongiu, F.) 101

Vergleichende Untersuchungen mit dem Radio-Bengalrosa- und dem Bromsulphalein-Test ( U t h g e n a nnt, H., Dah l, P., Piening, O.) 211

Die Lungenszintigraphie als Mittel zur Früherkennung des Lungenkrebses (O e s e r, H., Ernst, H.) 333

Die Telecaesiumbehandlung der Larynx- und Hypopharynxkarzinome ( $\mathrm{K} \mathrm{a} \mathrm{u} \mathrm{f} \mathrm{m} \mathrm{a} \mathrm{n} n, \mathrm{H}$.) 339

Hirntumor-Nachweis mit Radioisotopen ( $\mathrm{S} \mathrm{c} \mathrm{h} \mathrm{n} \mathrm{e} \mathrm{i} \mathrm{d} \mathrm{e} \mathrm{r,} \mathrm{C.)} 454$

Beschränkung des Röntgenologen auf sein Fachgebiet ( $\mathrm{c}$ h melcher, R.) 463

Beschränkung des Röntgenologen auf sein Fachgebiet auch im kassenärztlichen Bereich - EKG gehört nicht zum Fachgebiet des Röntgenologen (S c h melc her, R.) 513

Der ${ }^{131} \mathrm{~J}$-Trijodthyronintest und seine Kombination mit der mikrochemischen PBJ-Bestimmung im endemischen Kropfgebiet Südbadens (M e i ge n, B., Mert z, D. P.) 541

Morphologische Diagnostik der Leber mit Radioisotopen

(S c h n e id e r, C.) 819

Angiographie bei Beckenarterienverschlüssen zur Indikationsstellung für rekonstruktive Gefäßoperationen (B erghaus, H., Ka uf m a n n, H.) 894

Morphologische Pankreasdiagnostik mit Radioisotopen ( $\mathrm{S} \mathrm{h}$ n eider, C.) 1122

Strahlenschäden durch Verletzung der ärztlichen Sorgepflicht und Grundsätze zum Schmerzensgeldanspruch (S c h m e l c h e r, R.) 1058

Die klinischen Symptome der Hyperthyreose und ihre Bedeutung für die Diagnostik (Gü n ther, R., $\mathrm{L}$ a u b ing e $r$, G.) 1144

Umsatzsteuerpflicht des Krankenhausröntgenologen im Durchgangsarztverfahren ( $\mathrm{Sch}$ elcher, R.) 1169

Lymphangioadenographie des retroperitonealen Lymphsystems

(Belt $z$, L., Thurn, P.) 1177 


\section{Verdauungs- und}

\section{Stoff wechselkrankheiten}

Mákroradiógraphie mit "Radiophosphor in der Diagnostik de's Magenkarzinoms ( $\mathrm{H}$ a f t e r, E.) 90

Untersuctiungen zur Wirkung der Ascorbin'säure auf den Kohlenhydratstoffwechsel (Me h n e r t, H. För s ter, H., Fun ke, U.) 106

Kaliumhaltige Arzneimittel und stenosierende Dünndarmgeschwüre (W e llm a n n, K. F.) 131

Die Bestimmung der Adenosindesaminase im Serum. Prüfung diagnostischer Möglichkeiten

(Müller-Beissenhirtz, W. $\mathrm{K}$ e $1 \mathrm{l}$ e'r, H.) 159

Einfluß des sympathiko-adrenalen Systems, auf Herz-Kreislauf-Regulation und unveresterte Fettsäuren des Blutserums (K r.zy w a n ek, H. I, R a a b, Wo., B. h l e, E.) 193

Vergleichende Untersuchungen mit dem Radio-Bengalrosa- und dem Bromsulphalein-Test

(Uthgenannt; $H_{t}$ Dahl, P. Pi ening., O.) 211

Die Behandlung des Leberkomas (Martini, G. A.) 221

Fabry-Krankheit (Angiokeratoma corporis diffusum universale) Phosphatidspeicherkrankheit bei , zwei Familien

(D u b a c h, U. C., G loor, F.) 241

Die Bedeutung der Serumlabilitätsproben in der modernen Leberdiagnostik (Li:n d n e $r_{i}, H$.) 267

Weisen Xanthelasmen am Augenlid auf eine familiäre Hypercholesterinämie hin? ( $\mathrm{B} \mathrm{r}$ a u n - F a l c o, O.) 282

Mủ bei histaminrefraktärer Anacidität mit bestimmten Resorptionsstörungen, zum Beispiel von Spurenelementen gerechnet werden? (D r u b e , H. Ch.) 283

Welche Modifikation des Bromsulphaleintests hat sich am besten bewährt? (K r e u t z, F. H, 283

Uber eine seltene Form von. Ileus nach Magenresektion (Kol e, E.A., va n Deurzen, W: A.) 303

Die Kardiafunktion im Verlaufe der Kindheit (D i t t r i c h, J. K.) 308

Technik moderner Sekretionsanalysen der Magenschleimhaut (K r e n.t z, K.) 356

Die Therapie der Hyperkaliämie (F i i p p in i, L.) 359

Wie ist das Auftreten eines Diabetes mellitus bei Leberzirrhose zu erklären? (Creutz f eld, W.) 377

Hat die Bestimmung der $\alpha$-Amylase Vorteile gegenüber der Diastasebestimmung nach Wohlgemuth in der Pankreasdiagnostik? ( K e lIe r, H.) 377

Welche Leberfunktionsproben sind als Suchtest bei unklaren Lebererkrankungén zu empfehlen? (D e m lin g, L:) 378

Die Harnpufferanalyse in der Diagnostik und Therapie metabolischer Störungen des Säure-BasenHaushalts ( $\mathrm{S}$ o m m e r k a m p, $\mathrm{H}$., $\mathrm{K}$ l e t $\mathrm{t}, \mathrm{H}$.) 403

Welche Sonde ist zur Tamponade blutender Osophagusvarizen zu bevorzugen? (Haemmerli, U.P.) 422
Glykogenose Typ I (v Gierke) früher mitgeteilt als familiäre Hyperlipidämie (F u hrma n n, W., Jak o:v cic, $S, Y i$ Yung H sia D.) 453

Darf die Glucocorticoidtherapie einer chronischen Hepatitis oder Leber zirrhose bei nach gewiesenen Osophagusvarizen fortgesetzt werden? $(\mathrm{K}$ ü $h, \mathrm{H}, \mathrm{A}) 466$

Welche Zucker sind physiologisch am wichtigsten? (M e h n e r t, H.) 467

Laboratoriumsbefunde bei Anorexia nervosa (F r a h m, H.) 499

Der ${ }^{131} \mathrm{~J}$-Trijodthyronintest und seine Kombinátion mit der mikrochemischen PBJ-Bestimmung in endemischen Kropfgebiet Südbadens (Mergen, B., Mertz,D.P.) 541

Die Zytologie des Duodenalsaftes (W i t te, S.) 548

Technik des Xÿlosetests? ( B ü t t n e $r, H$.) 562

Die diagnostische Bedeutung sekretorischer Funktionsanalysen! der Magenschleimhaut: (Krie n t $\mathrm{z}, \mathrm{K}$.) 596

Probleme der renalen Säure-BasenRegulation (Pabst, K, Ge rok, W. B a u m, P.) 600

Wirkungsort und -mechanismus der Lipoproteidlipase? (Bra un s te i n e $r, H$ ) 610

Zur Frage der konservativen oder operativen "Behandlung von Pankreasfisteln ( $\mathrm{K}$ üm m e r l e $\quad F$. M a p p e s, G.) 643

Selektive Vagotomie und Pyloroplastik in der Behandlung des $\mathrm{Ga}$ stroduodenalulkus und der Gastritis haemorrhagica (A llgö wer M., Heg g l i i., J.) 648

Erhebungen über Atiologie; Pathogenese, Therapieerfolge und Uberlebenszeit an einem unausgewählten Krankengut von 560 Patienten mit Leberzirrhose

(Creutzfeldt, W., Beck,K.) 682

Der labile Diabetes. Pathophysiologie, Klinik, Therapie (P e trides, P.) 689

Die Leberzirrhosen. Revision eines vielschichtigen Problems (Thaler, H.) 733

Die endokrine Beeinflussung des Stoffwechsels im Fettgewebe (L ö f'fler, G., W e ing es, K. F.) 773

-Werden Pleuraergüsse als Folge von Pankreaspseudozysten häufiger beobachtet? (H e s s , W.) 783

Unterschiede der Diastasewerte im Serum und Harn? (B ü $t \mathrm{t} n$ e $r, H$.) 784

Morphologische Diagnostik der Leber mit Radioisotopen ( $\mathrm{c}$ h n e i de $r$, C.) 819

Technik der Stuhlfettbestimmung nach van de Kamer? (B üt tn err, H.) 829

Injektionsbehandlung des Hämorrhoidalleidens? ( $\mathrm{R}$ os $\mathrm{Ch} \mathrm{ke,} \mathrm{W.)}$ 829

Störungen nach Magenresektion (H enning, N., Berg, G., Wüst, H., $\mathrm{Z}$ e i t l e r, G.) 843
Kann durch die Arbeit am Elektroschweißgerät eine Magenkrankheit begünstigt werden? (S y m a n s k i H. J.) 872

Welche Kriterien bei Blut- und Urinuntersuchung erlauben die differentialdiagnostische Abtrennung $\mathrm{v}$ funktioneller Hyperbilirubinämie hämolytischem Ikterus und posthepatischen Leberschäden? (M a r ko f $f$, N. G.) 919

Uber die Wirkung eines neuen Depot-Biguanids auf den Kohlehydratund Eiweißstoffwechsel bei Diabetes mellitus (M ü ti $\mathrm{n} \mathrm{g}$, D.) 939

Die Therapie der akut intermittierenden Porphyrie (Filippini, L.) 959

Die solitäre Exulceratio simplex (Dieulafoy) als Ursache massiver Intestinalblutungen ( $\mathrm{St} \mathrm{r}$ e i c her H. J.) 991

Die chirurgische Behandlung der Colitis ulcerosa (D e u c h e r, F.) 1001

Indikationen und Grenzen der Gastroskopie (O t t e n j a n n , R:) 1045

Führt längere Einnahme von Diphenylhydantoin zu gastrointestinalen Störungen wie Diarrhoen oder Duodenalulzera? (D rube, H.Ch.) 1061

Uber die Regulation der unveresterten Fettsäuren unter besonderer Berücksichtigung der Prostaglandine (B öhle; E., D itschuneit, H., Melani, F., Beyer, J., S chöf fling, K., Pf eiffer, E. F.) 1083

Ikterus als Symptom einer Spätgestose (M $\ddot{\mathrm{u}} \mathrm{h} l \mathrm{l}$ e $\mathrm{r}, \mathrm{E})$.

Ulcus ventriculi dissecans ( $\mathrm{D}$ o b r o wolski, Z. Golab W., Szy s z k o, St:) 1121

Morphologische Pankreasdiagnostik mit Radioisotopen ( $\mathrm{S}$ c h n e i d e r C.) 1122

Zur diätetischen 'Cystinosebehandlung (B a u e $r$, B.) 1124

Ist bei der Magensaugbiopsie die manuelle Aspiration oder die automatisch gesteuerte Ansaugung zu bevorzugen? ( O t ten j an $n$, R.) 1134

Wie hoch ist der Methionin- und Cholingehalt von Quark (Leberdiät)? $(\mathrm{N}$ o e $11 \mathrm{e}, \mathrm{H}$.) 1134

Die heutige Stellung der Laparoskopie in der Leberdiagnostik (L i n d n e r, H.) 1160

Gibt es Aquivalente eines Gichtanfalles, die eine Angina pectoris vortäuschen und im Elektrokardiogramm flüchtige Symptome eines Infarktes zeigen? ( $\mathrm{Z}$ ölln e r, N.) 1171

Gibt es eine einfache Methode zur Bestimmung von Chymotrypsin im Stuhhl? (A m m a n, R.) 1172

\section{Verschiedenes}

Die Regelung der Koronardurchblutung ( $\mathrm{H}$ i r c h e, Hy.) 1

Differenzierung der essentiellen familiären Hyerlipoproteinämien (Hyperlipidämien) (Ka h I k e, W.)

Entzündungsstoffe (F r i m m e r, M.) 
Welchen Einfluß haben verschiedene Sportarten auf juvenile Kyphosen? ( $R$ a t h k e, F. W.) 44

Zur Beeinflußbarkeit von Choriokarzinomzellen durch Zytostatika und Immunkörper in der Gewebekultur (H e c k m a n n, U., T r a n e k jer, S.) 66

Uber die Neuroanatomie in der Medizin won heute (Fi l is c h h a er, K) 75

Nachweis von Barbitursäure-Derivaten im Harn (F ra $\left.h_{1}, M_{\text {: }}\right) 81$

Die thymoiden Organe des Immunitätssystems ( $\mathrm{G}$ ü $\mathrm{n}$ t h e $r$, O.) 87

Wie kann man im Saugbiopsiematerial die Tumorzellen von störenden Erythrozytenbeimengungen isolieren ohne die phasenkontrastzytologische Beurteilbarkeit $\mathrm{zu}$ beeinträchtigen? (S e if e r t , G.) 95

Ist die Blutzuckerbestimmungsmethode nach Crecelius-Seifert in der Praxis brauchbar und zuverlässig? (Mehnert, H., Förs ter. H.) 95

Methodische Hinweise zur Diagnostik von Chromosomen-Anomalien (S to e ckenius, M.) 125

Karzinogenese im Lichte der Statistik. Bemerkungen zum Beitrag von $\mathrm{K}$. Freudenberg in dieser Wochen. schrift $90,(1965), 944$, S G h in z, H. R., R e i c h, Th.) 135

Karzinogenese im Licht der Statistik. SchluBwort (E r e u d e n b e r g ${ }_{i} \mathrm{~K}$.) 137

Beeinflußt, Alkoholgenuß die Kalkstickstoffvergiftung? (Hos chek , R.) 138

Die Bestimmung der Adenosindesaminase im Serum. Prüfung diagnostischer Möglichkeiten ( $M$ ül. ler-Beissenhirtz, W.o Kelle $r, H)$,

Zum Wirkungsmechanismus der Steroidhormone

(D rew S, J., W a cke,r, A.) 178

Ist Streckentauchen oder Tieftauchen gesundheitssçädlich? (Holl ma nn, W.) 187

Vergleichende Untersuchungen mit dem Radio-Bengalrosa- und dem Bromsulfalein-Test

(Uthgenannt, H., Dahl, P., Piening, O.) 211

Laboratoriumsbefunde bei chronischentzündlichen Rheumaerkrankungen (Moll, W.) 219

Gibt es, außer dem eigenen Willen, wirksame "Nicótin-Entwöhnungsmittel ( $\mathrm{K}$ u s ch in s ky ; G.) 233

Welchen Einfluß hat die Einwirkungsdauer- und -temperatur der Coffeinlösung bei der Bilirubinbestimmung nach Jendrassik: und Cleghorn? (B üt t n e r , H.) 233

Die Bedeutung der Serumlabilitätsproben in der modernen Leberdiagnostik (L i n d n e r, H) 267

Welche Modifikation des Bromsul. phaleintests hat sich am besten bewährt? (K r e u t z, F. H.) 283
Derzeit gültige Normgrenzen für Mi. neralien und andere wichtige Substanzen im 24-Stunden-Harn? (D u l c e , H.-J.) 325

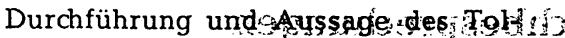
butamid-Tests? (M e h n e r t? $\mathrm{H}$.) 326

Technik moderner Sekretionsànalýsen der Magenschleimhaut

(Kin e nt $z, K) 356$

Grundlagen der Lymphozytenkultur

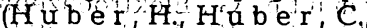
B raunsterner, H.) 360

Hat die Bedeutung der $\alpha$-Amylase Vorteile gegenuber der Diastasebestimmung nach Wohlgemuth in der Pankreasdiagnostik? ( $\mathrm{Kel}$ le $\mathrm{r}, \mathrm{H}$.) 377

Glykogenose Typ I (v, Gierke) fruher mitgeteilt als familiăre Hyperlipidämie (Fuhrmann, W.Jakovc.i.c. S., Yi Yung Hsia, D.) 453

Uber die Diagnostik und Therapie der, erblichen Lese-RechtschreibeSchwäche (We ins chénk, C.) 461

Darf die Glucocorticoidtherapie einer chronischen Hepatitis oder Leberzirrhose bei nachgewiesenen Osophagusvarizen fortgesetzt werden? (K üh h, H. A.) 466

Zur Frage der Magenspülung bei Vergifteten ( $B$ alzereit, F A r nold, W.) 485

Die Zytologie des Duodenalsaftes (W i t t e, S.) 548

Technik des Xylosetests? (Büt $t$ n e r, H.) 562

Was ist über die Synchronisation vegetativer Funktionen mit rhythmisch einwirkenden Reizen bekannt (A s, ch of f, J.) 610

Zytogenetik des Plasmas (R ö b bele $n, G)$.

Soll bei Linkshändern in der Schule der Versuch einer Umstellung auf Rechtshändigkeit : durchgeführt werden? (H e li b u u ge, Th.) 783

Unterschiede der Diastasewerte in Serum und Harn (B ü tt n er, $H_{\text {. }}$ ) 784

Welche Vorteile hat die Verstärkerauskultation ( $\mathrm{H}$ o $1 \mathrm{l} \mathrm{d} \mathrm{a} \mathrm{c} \mathrm{k,} \mathrm{K.)} 829$

Zum Problem der Infektion des Menschen,' insbesondere des Kindes, mit Larven von Tier-Askariden (S choop, G., L a mi na, J) 848

Untersuchiungen zur Haltbarkeit der Ferment-Aktivităt inı Serum

(A melung, D., Hoff m a n n, L., O t t o, L.) 851

Xann durch die Arbeit am Elektroschweißgerät eine Magenkrank. heit begünstigt werden? ( $\mathrm{S}$ z Y mag s. ki,H.J.) 872

Bestehen Erfahrungen in der Anwendung von Corticosteroiden bei Höhenkrankheit beim Bergsteigen? (S chwalb, H.) 873
Allergiediagnostik beim Asthma bronchiale (Gron emeyer, W.) 902

Stufenbelastung zur Beurteilung der korperilcnen Leistungstanigkeit u. der Koronarreserve ( $\mathrm{K}$ a l te n. b a c h, M.) 884

Spezifische Desensibilisierung mit Inhalationsantigenen: ( $F, c_{1}, S, E$.) 904

Klinische Physiologie. Entwicklung, Organisation und Aufgaben dieses Fachgebietes in Schweden (Gra f , K., Stt.rö mi, G.) 911.

Welche Kriterien bei Blut-, und Urinuntersuchungen erlauben die differentialdiagnostische Abtrennung v. funkitioneller Hyperbilirubinämie hämolytischem Ikterus und posthepatitischen Leberschäden? ( $\mathrm{M}$ a $\mathrm{r}$ $k$ of $f, N$. G.) 919

Ist: es ärztlich vertretbar Milch aus eigenem, gesundem (Tuberkuloseund Brucellose-freiem) Stall ohne Abkochen oder Pasteurisieren abzugeben? (E y e r. H.) 919

Synacthen-Kurztest zur Beurteilung der Nebennierenrindenfunktion (O t to, H., M i n n e ke r, C., S p a e:t h, R.) 934

Die Infrarotthermographie in der $\mathrm{Kli}$ nik ( $\mathrm{B} \mathrm{r}$ à n e m a r k, P. I.) 961

Kann ein Kurzwellentherapiegerät eine : im gleichen Raum stattfindende andere Elektrotherapie stö-

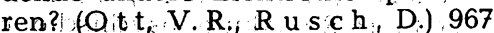

Ist es bei Befunden des klinischen Laboratoriums sinnvoll u. notwendig, Normalwerte anzuführen? (B ü t $t$. ner, H, 1061

Chromosomenanomalien als Abortutsache ( P a r low i tzki， I. H.) 1094

Besondere klinische Formen des Thymus-Syndroms (B ü chner, M., Gehrman $n$, G.) 1105

Inwieweit wird die Blutsenkung durch eine Hypercholesterinämie Hypertriglycéridämie oder ganz allgemein dúrch Hyperlipämien beeinflußt? (S ch e t t 1 er, G.) 1133

Die heutige Stellung der Laparoskopie in der Leberdiagnostik (L indner, H.) 1160

Zur Bedeutung des regeltheoretischkybernetischen Konzepts in der Medizin (D ris c he $1, H$.) 1164

Gibt es eine einfache Methode zur Bestimmung von Chymotrypsin im Stuhl? (A m m a n, Ri) 1172

Diagnose der Polyneuropathien (J a n z n , R.) 1192

Therapie bei Polyneuropathien (B a l z e r e it , F.) 1194

Wandlungen der Ehrlichschen Seitenkettentheorie ( $\mathrm{G} \ddot{\mathrm{u} n \mathrm{th}} \mathrm{e} \mathrm{r}, \ldots \mathrm{O}$.) 11.97

Ursache des Jodmangels in manchen Gegenden der Alpenländer? (E y e r, H.) 1202 


\section{Buchbesprechungen}

A e bi, H.: Einführung in die praktische Biochemie ( $\mathrm{S}$ t a u dinger) 380

A lken, C. E., D i x, V. W., W e y $\mathrm{r}$ a u c h, H. M.: Handbuch der Urologie Band VII/2 ( $\mathrm{M}$ a y) 564

A m b r o s e, E. J.: Cell electrophoresis ( $\mathrm{S}$ a c h t l e b e n) 1007

B a b i c s, S., R é n y i - V ám os, F.: Clinical and theoretical pictures of some renal diseases ( $\mathrm{B} \mathrm{u} \mathrm{C} \mathrm{h}$ b o r $\mathrm{n}$ ) 1173

B a d e r, G.: Die viszeralen Mykosen (S e eliger) 787

B a h r, G. F., Z e it l e r, E. H.: Quantitative electron microscopy (S a ndrit ter und $K$ öp p e l) 875

$\mathrm{B}$ á 1 i n $\mathrm{t}$, P.: Nierenclearance (B u c h bor n) 1211

Baron, D. N.: Essentials of chemical pathology ( $\mathrm{K}$ a r l s o n) 564

B a u li eux, E. E., Robe l, P.: Aldosterone ( $\mathrm{K}$ r ü c k) 284

B e c k m a n n, R.: Myopathien (Erbs ö h) 45

B ertram, F., Otto, H.: ABC für Zuckerkranke ( $\mathrm{M}$ i c h e l) 516

B le uler, M., Willi, J., B üh ler, H.R.: Akute psychische Begleiterscheinungen körperlicher Krankheiten (S c h u l t e) 968

B o ck , K. D.: ABC für Hochdruckkranke (Gill m a n n) 920

Brand, G., Kunz, H., $\mathrm{N}$ is s en, R.: Intra- und postoperative $\mathrm{Zwi}$ schenfälle. Bd. II: Abdomen (Z e n $\mathrm{k}$ e r) 283

Brazier, M. A. B.: Brain function. Vol. II. (H a r b e r s) 516

Brosig, W., Nage l, R.: Nierentransplantation (G a c a) 1007

B r o w n ing, E.: Toxicity and metabolism of industrial solvents ( $\mathrm{He} \mathrm{n} \mathrm{s} \mathrm{ch} \mathrm{l} \mathrm{e} \mathrm{r)} 668$

B r ü ckn er, R.: Augenfibel (Holl w i c h) 1062

B r ü h l, W.: Leber- und Gallenwegserkrankungen (B a r t e l hei mer) 1099

Bundes-Seuchengesetz 4. Ergänzungslieferung ( $\mathrm{S} \mathrm{c} \mathrm{h} \mathrm{m} \mathrm{e} \mathrm{l} \mathrm{c} \mathrm{h} \mathrm{e} \mathrm{r)} 380$

B u r c k, H.-Chr.: Histologische Technik (L e o n h a rd t) 1135

Co I m a n t, H. J.: Zerebrale Hypoxie ( $\mathrm{H} \mathrm{e} \mathrm{y} \mathrm{c} \mathrm{k)} 423$

Committee on Trauma: The management of fractures and soft tissue injuries (W e 11 e r) 668

Crews, E. R.: A practical manual for the treatment of burns (I p p e $n$ ) 1063

Davidson, J.N., Cohn, W. E.: Progress in nucleic acid research and molecular biology (Harbers) 379

Defoe, D.: Ein Bericht vom Pestjahr London 1665 (S c ha de w a ld t) 1063

Diethelm, L., Olsson, O. Strnad, F., Vieten, H., Z u p pinger, A.: Handbuch der medizinischen Radiologie. Band $\mathrm{X}$ (D e c h e r) 468
Doctor X: Intern (Wellma n n) 727

D üb e n, W.: Der Arzt am Unfallsort ( $\mathrm{J}$ ung h a n $\mathrm{s}, \mathrm{H}$.) 564

D u m e r m u t h, G.: Elektroenzephalographie im Kindesalter (S t e p h a n) 187

E a r l, A.S.: Surgery in America ( $N$ is s e n) 874

Egenter, R., Matussek, P. Ideologie, Glaube und Gewissen (E n g e l m e i e r) 424

Ehrhardt, H.: Euthanasie u. Vernichtung "lebensunwerten" Lebens (S c h e t t le r) 236

E t m e r, F., B o l c k, J.: Arzneimittelgesetz. 6. Ergänzungslieferung (L e n d l e) 668

Eyermann, E., Fröhler, L.: Verwaltungsgerichtsordnung

(S chmelch e r) 833

F a w c e t $t, D$. W.: The cell. Its organelles and inclusions (W e llm a n n) 1212

Feurstein, V.: Grundlagen und Ergebnisse der Venendruckmessung zur Prüfung des zirkulierenden Blutvolumens ( $\mathrm{J}$ u s t) 1211

Frehner, H. U.: Diabetes-Fibel (P f e iffer) 467

G a b ler, G.: Myocardose-Myokarditis (B l o c h) 1135

Gercke,W., Düllo, H., Böckel, E.: Medizin im Sozialrecht. 34. bis 39. Ergänzungslieferung (S c h m e l c h e r) 876

Gesellschaft für Physiologische Chemie - 14. Colloquium am 25./27. 4 1963 Mosbach: Mechanismen enzymatischer Reaktionen (K a r l s o n) 786

Glocker, R., Macherauch, E.: Röntgen- u. Kernphysik (Ga u w e r k y) 234

Goerttler, K.: Die menschliche Glatze im Altersformwandel der behaarten Kopfhaut ( $\mathrm{K}$ a n t n e r) 785

Good, R. A., Ga briels en, A. E. The thymus in immunobiology (V orla en d e r) 47

Got tron, H. A. S c hön f e ld, W. Dermatologie $u$. Venerologie. Bd.V. Teil 2 (S t e i g l e d e r) 95

Gstirner, F.: Chemisch-physikalische Vitamin-Bestimmung ( $\mathrm{S}$ t r o h e c k e r) 876

$\mathrm{H}$ a a s, R., Vivell, O.: Virus und Rickettsieninfektionen des Menschen (B o n i n) 423

H a cks, S.: Schmerzensgeld-Beiträge ( $\mathrm{S} \mathrm{ch} \mathrm{m} \mathrm{e} \mathrm{l} \mathrm{c} \mathrm{h} \mathrm{e} \mathrm{r)} 787$

$\mathrm{H}$ a ll er, J.: Ovulationshemmung durch Hormone (D ö r i n g) 830

$\mathrm{H}$ arder, H. J.: Technische Sicherheitsprobleme im Operationstrakt ( $\mathrm{J} \mathrm{u} \mathrm{s} \mathrm{t}) 47$

Harper, H. A.: Review of physiological chemistry ( $\mathrm{K}$ a r l s o n) 1062

$\mathrm{H}$ a y hoe, F. G. J.: Current research in leukaemia. A symposium (R e m y) 235
Heffter, A., Heubner, W. E i c h ler, O., F a r a h, A.: Handbuch der experimentellen Pharmakologie. Band 16, Teil 9 (K o I l) 46

Heilmeyer, L., Holtme ì er, H. J.: Hochdruckforschung (S c h n e id e r) 563

Heffter, A., Heubner, W. E i c h l e r, O., Far a h, O.: Handbuch der experimentellen Pharmakologie. Bd. 17: Ions alcalino-terreux. Teil 1: Systèmes isolés ( $\mathrm{K}$ oll) 97

Heinemann, G. W., Li e bold R.: Kassenarztrecht. 4. Aufl. 14. Lieferung (S c h m e lc h e r) 1100

Henry, R. H.: Clinical-chemistry (I p p e n) 424

Heupke, W.: Zuckerkrank-Behandlung und Diät (Robbers) 1135

Hoi g né, R.: Arzneimittel-Allergien (K us chin s k y) 727

Holz m a n n, M.: Klinische Elektrokardiographie ( $\mathrm{Hegglin,} \mathrm{R.}$ B oll ing e r, A.) 874

Hop pe-Seyler/Thierfelder: Handbuch der physiologischen und pathologisch-chemischen Analyse Bd. VI, Teil A: Enzyme (Hess) 1100

Hornof, Z., Schmid, L.: Poumon, Respiration et Sport ( $\mathrm{K} \circ \mathrm{c} \mathrm{h})$ 1212

Hübenert, J., Sta i b, W. H. Biochemie der NebennierenrindenHormone ( $\mathrm{K}$ a r l s o n) 515

$\mathrm{H}$ u e c k, A.: Kündigungsschutz (S c h m e l c h e r) 1063

$\mathrm{J}$ a k o b ovit s, A.: Endokrinologie des Ovars (S t a e m m le r) 831

$\mathrm{J}$ a c c h i a, E.: Atom-Sicherheit und Rechtsordnung (L o s s e n) 1136

J o n a s ch, E.: Unfallchirurgische Operationslehre (W e $1 \mathrm{l}$ e r) 564

$\mathrm{J} \mathrm{u} \mathrm{cker,} \mathrm{E.:} \mathrm{Progress} \mathrm{in} \mathrm{drug} \mathrm{re-}$ search (L e n d l e) 46

$\mathrm{K}$ a iser, H.: Cortisonderivate in Klinik und Praxis (F le is ch h a c k e r) 46

$\mathrm{K}$ a is er-Mein hard t, I.: Atlas der Ohren-, Nasen- und Halskrankheiten sowie deren Grenzgebiete (B i e s a ls k i) 327

Karls o n, P.: Kurzes Lehrbuch der Biochemie (S t a u d in g e r) 920

Kass, E. H.: Progress in Pyelonephritis (L u tze y e r) 1006

Kaverina, N. V.: Pharmacology of the coronary circulation (L u 11 . m a n n) 727

Keid e r li n g, W.: Grundlagenforschung in ihrer Bedeutung für die klinische Medizin (We i s b e c k e r) 832

Kelemen, E.: Physical diagnosis of acute abdominal diseases and injuries (Hor n b o s t e l) 424

Keliki a $n$, H.: Hallux valgus, allied deformities of the fore-foot and metatarsalgia (E x n e r) 831 
Kelle r, W., Wis k o t $t$, A.: Lehrbuch der Kinderheilkunde (W i n dorfer) 563

Ke ut h, U., Heggli n, R., L e u t hardt, F., Schoen, R., S c h wi e g k, H., Z ollinger, H. U.: Das Membransyndrom der Früh- und Neugeborenen (W i n dorfe r) 423

Kirch h of $f, H_{\text {., }} \mathrm{Kräubig}, H_{\text {., }}$ Anders, W., B ock, J., Es sbach, H., Kabelitz, H. J., Langer, H., Piekarski，G., Remky, H., Röse, I., Thalhammer, O., Werner, H.: Toxoplasmose (Win dorfer) 1006

Klausberger, E. M.: Die Darstellung von Hirndurchblutungsstörungen durch die cerebrale Angiokinematographie (Krayen b ü h l) 968

Klepzig, H.: ABC für Herz- und Kreislaufkranke (F r a n k e) 379

Klepzig, H., Frisch, P.: Die röntgenologische Herzvolumenbestimmung in der Praxis (L o o g e n) 327

Kory, R. C., T s a g ris, T. J., Bustamente, R. A.: A primer of cardiac catheterization (B a y e r) 516

$\mathrm{Kranz}, \mathrm{H} ., \mathrm{H}$ ein $\mathrm{rich}, \mathrm{K}$ : $\mathrm{Be}-$ gleitwirkungen und Mißerfolge der psychiatrischen Pharmakotherapie (Ki el holz) 437

Krayenbühl, H., Yasargil, M. G.: Die zerebrale Angiographie (Z ü lc h) 726

Kreuzer, A.: Arztliche Hilfeleistungspflicht bei Unglücksfällen. (S c h m e l c h e r , Karlsruhe) 1212

K r u p p, M. A., S w e e $t, N$. J., J a we tz, E., Biglieri, E. G.: Physicians Handbook ( $\mathrm{H}$ or $\mathrm{n}$ b os te $\mathrm{l}$ ) 469

Kühn, A.: Vorlesung über Entwicklungsphysiologie (Hodler) 1007

$\mathrm{Kap}$ fers c h m id t, H. G.: Englisch für Mediziner (L e j e u n e) 1137

$\mathrm{Kusch}$ in s k y, G.: Taschenbuch d. modernen Arzneibehandlung (B o c k) 516

Kusch insky, G., Lüllmann, H.: Kurzes Lehrbuch der Pharmakologie (L e n d l e) 611

Laborit, H.: Les regulations métabolique (F r e y) 832

L a m b , L. E.: Electrocardiography and vectorcardiography ( $\mathrm{H}$ ol $\mathrm{A}$ $m$ a n n) 1172

L a nge, M.: Lehrbuch der Orthopädie und Traumatalogie. Band II. (R ü t t) 187

L a n $\mathrm{g}$, M.: Lehrbuch der Orthopädie und Traumatologie. II. Bd. 1. Teil ( $\mathrm{H}$ o h m a n n) 284

L a u benthal, F.: Sucht und Mißbrauch (B o c k) 327

La uber, H. L.: Das Pneumencephalogramm. Meßverfahren bei Erwachsenen (M ü 1 l e r) 188

L a w k o w icz, W., Krzem in s k a - L a w k o w i c z, I.: Differentialdiagnose hämatologischer Erkrankungen (Braunsteiner) 667

L e m b e ck, F.: 1 x 1 des Rezeptierens ( $\mathrm{K} \mathrm{u} \mathrm{s} \mathrm{c} \mathrm{h} \mathrm{in} \mathrm{s} \mathrm{k} \mathrm{y)} 423$
L i n n e w e h, F.: Fortschritte der Pädologie ( $W$ in d or f e r) 786

$\mathrm{L} \ddot{\mathrm{u}} \mathrm{t} \mathrm{h}, \mathrm{H}$.: Schöpfungstag u. Mensch der Zukunft (F u hrman n) 1173

Luthe, W.: Autogenes Training (B r äu t i g a m) 379

Markowitz, M. A. B., Kuttner, A. B. S.: Rheumatic fever, Diagnosis, management and prevention (Ho e n) 328

McIntyre, N., Sherlock, S.: Therapeutic agents and the liver ( $\mathrm{K} \ddot{\mathrm{u} h \mathrm{~h}}$ ) 188

Meites, S.: Standard methods of clinical chemistry (B üt t n e r) 235

Melnick, J. L.: Progress in medical virology. Vol. 7 (B o n i n) 96

Me n n e, F., L a n g e, K.: Grundriß der Chemie und klinischen Chemie Bd. I ( $\mathrm{B} \ddot{\mathrm{u}} \mathrm{t} \mathrm{t} \mathrm{n}$ e r) 284

Miescher, G. $\Psi$, Storck, H.: Handbuch der Haut- und Geschlechtskrankheiten. II. Bd./2. Teil (Got tron) 1062

Mikat, B.: Die Tuberkulosehäufigkeit in den Kreisen der Bundesrepublik Deutschland im Jahre 1958 (Gries b a c h) 283

Mitchell, J. S.: The treatment of cancer (B o c k) 783

Mitchell, R. S.: The pathogenesis of the chronic obstructive bronchopulmonary disease (M a r x) 876

Mör l, F.: Lehrbuch der Unfallchirurgie (Kraus s) 468

Mo e s chli n, S.: Therapie-Fibel $96^{*}$

Møller, K. O.: Pharmakologie (K u s c hin s k y) 1211

Müller, G.: Die Salmonellen im Lebensraum einer Großstadt (S e e lig e r) 48

Musshoff, K.: Differentialdiagnose seltener Lungenerkrankungen im Röntgenbild ( $\mathrm{H}$ e y m e r) 235

Naumann, R. L., $N$ öbel, L., Siebelist, I., Starke, G.: Virologische Praxis (B o n i n) 1063

$\mathrm{Nigst}$, H., Ba um an n, E.: Spezielle Frakturen- und Luxationslehre. Bd. II, Teil 1: Ellbogen (W e lle r) 234

Noetzel, H., Jerusa lem, F.: Die Hirnvenen- und Sinusthrombosen ( $\mathrm{Kling} \mathrm{le} \mathrm{r)} 328$

$\mathrm{Nults} \mathrm{ch}, \mathrm{W} .:$ Allgemeine Botanik (B r a u n e r) 726

$\mathrm{Obeck}, \mathrm{V}$. : Isometrie (Het $\mathrm{t}$ n ge r) 517

$\mathrm{O}$ b e r, K. G., Mein re nk e n, H.: Allgemeine und spezielle chirurgische Operationslehre. IX. Band (K ä s e r) 188

O k r ös, S.: The heredity of papillary patterns (W e $\mathrm{n} \mathrm{d} \mathrm{t)} 969$

Opitz, H., Schmid, F.: Handbuch der Kinderheilkunde, Band IV (Künz e r) 1172

P a s t insky, I., R á cz, I.: Hautveränderungen bei inneren Krankheiten (I p p e n) 380

P in c u s, G.: The control of fertility (H a lle r) 874

Pitzen, P., Lindemann, K.: Kurzgefaßtes Lehrbuch der orthopädischen Krankheiten (Exner) 236
Porter, K. R., Bonneville, $M$. A.: Einführung in die Feinstruktur von Zellen und Geweben (B e r g m a n n) 611

Ra per, K. B., Fennell, D. I.: The genus aspergillus (Seeliger) 1173

R e ploh, H., Ot t e, H.-J.: Lehrbuch der mikroskopischen Mikrobiologie ( $R$ o e m e r) 875

R i c h t er, G. W., E p s te in, M. A.: Experimental Pathology (L e t t e re r) 328

R o b e r t s, J. C., S t r a us, R.: Comparative atherosclerosis (Schett. le r) 612

Scheid, W., Wieck, H. H. S t a m m ler, A., J o c h heim, K. A., Seidenfaden, I., Gibb e ls, E.: Lehrbuch der Neurologie (M u m e $\mathrm{th}$ a $\mathrm{l}$ e r) 968

$\mathrm{S} \mathrm{ch}$ ind le r : Fortschritte d. Krebsforschung. Vol. I (M o h r) 1136

S chinz, H. R., B a en s ch, W. E. Fromm hold, W., Glauner, R., Uehlinger, E., Wellauer. J.: Lehrbuch d. Röntgendiagnostik. Bd. I. Allgemeine Grundlagen und Methoden (Z dan s k y) 667

S chinz, H. R., B a ens ch, W. E. Frommhold, W., Glauner. R., U e h linger, F., Wellauer J.: Lehrbuch d. Röntgendiagnostik. Band V: Abdomen. ( $\mathrm{Z}$ d an s k y) 874

Schmidtke, H.: Die Ermüdbarkeit (Hos c h e k) 969

S c hnei d er, K.: Klinische Psychopathologie ( $\mathrm{W}$ i e c k) 785

S e i g e, K.: Diabetes mellitus (Mehnert) 187

S e t t e le, R.: Gesetz über die kommunalen Versorgungsverbände in Baden-Württemberg (S c h m e lch e r) 48

Siebert-Hilger: Arbeitsrecht (S c h m e lc h e r) 727

Siega l, L.J.: Forensic Medicine (P o n s o ld) 1172

Siegl, J.: Therapie der Kinderkrankheiten (W o $1 \mathrm{f}$ ) 235

Solom ons, B.: Lectures Notes on Dermatology (B r a u n-F a l c o) 1099

S m o l k a, H., S o o s t, H. J.: Grundriß und Atlas der gynäkologischen Zytodiagnostik (F r i ed b e r g) 563

$\mathrm{S} t$ e i $\mathrm{n}$ b e r g, A. G., B e a r n, A. G.: Progress in medical genetics. Vol. IV. ( $\mathrm{B}$ e c $\mathrm{k}$ e r) 830

Strehler, B. L.: Advances in gerontological research, Volume I. (L e t t e r e r) 283

Streiff, E. B.: Moderne Probleme der Ophthalmologie III (Hollw i c h) 920

Symposia for orthopedic surgeons ( $\mathrm{R}$ a t h k e) 786

Thoenes, W.: Mikromorphologie des Nephron nach temporärer Ischämie ( $R$ o t t e r) 468

Töndury， G.: Angewandte und topographische Anatomie (v. H e r r a t h) 378

Traumatologie in der chirurgischen Praxis (Kra us s) 667

Tünte, W.: Vergleichende Untersuchungen über die Häufigkeit menschlicher Mißbildungen (W e i c k e r) 1100 
W alt hier-Bücd, H., YS, po e r vir,

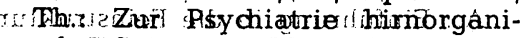

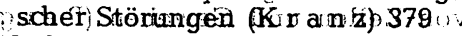

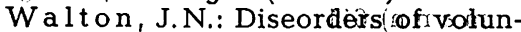

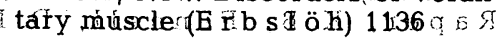

Wi e ble r, Cari Advances un enzyme regulation. Vol. 3. (A m elüing)

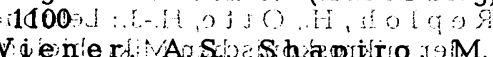

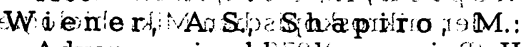
Advances in bloód(igrouping II

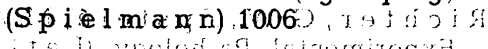

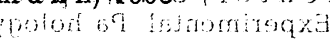

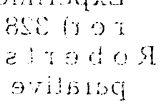

\section{Verhảndiungsberichte}

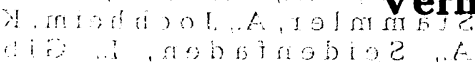

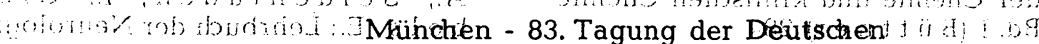

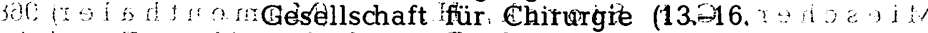

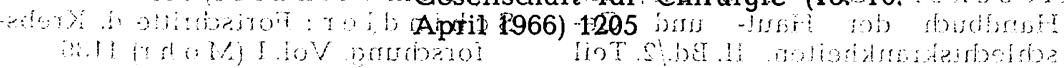

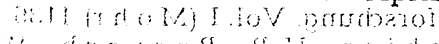

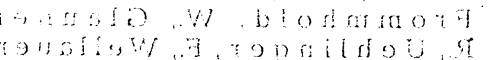

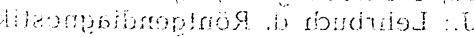

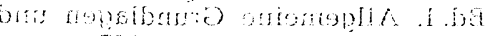
ud (y d a n b S Itoliondal

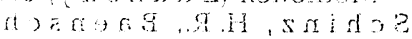

Wirk in son,roj.: H., is dsoenzymes

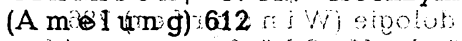

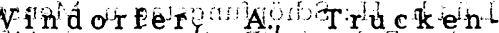
br od t, H.: Kinderárztliche Notfallfibel (Kut in ér) 422

Wint e $r, C, C$ Radioisotópe renor graphy (O f f 469

Wialstenhol merger hight J.: Complement (V or la a der)

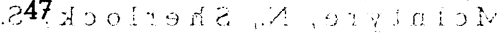

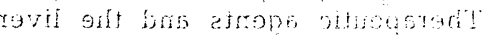

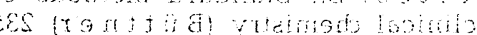

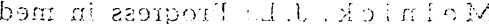

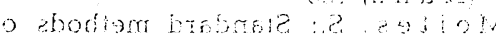

Wust row F. Die Tumoren des Gesichtsschadels (B b c ke r, W. 46

Yama to, W. S', Bro be ck, J.R.: Physiological controls and regulations (B r u c k) 1212

$\mathrm{Zweif} a \mathrm{ch}$ B. W. Grant in., M c Cluskey, R. T: The inflammatory progress (Fi $\mathrm{s}$. $\mathrm{h}$ \& $\mathrm{r}$ und

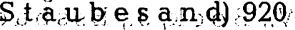

\section{A}

\section{Sachverzeichinis}

AB0-Blutgruppenverteit is a Adenoma sebaceum und lung 48 prij

ABC für Herz; und Kreis rirolo Adenosindesaminase im

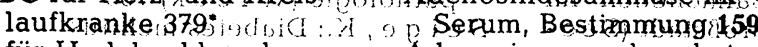

- für Hochdruckkranke

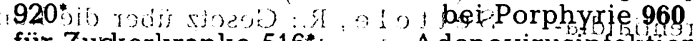

- für Zunckerkranke 516 to Adenovirusinfektion und

Abdomen, chirurgische in $/$ - F Facialisparese 256

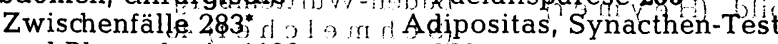
-i) und Pleyrodynie 1180 - 1 i a 936 .

-, Röntgendiagnostik, $874^{*}$ ) a Adrenalin und Féttsäurén

Abduktion bei Hüft- 193 is is je

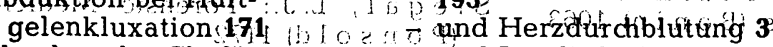

Abgaben der Chefärzte

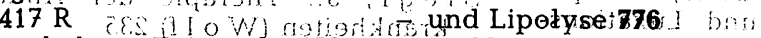

Abnabelung zejtpunkt 458 , in 7 im -Nervensystem 318

Abort bei Chromosamen ol w and Rezeptorenblocker anomalien 1094

- und Thalidomid 11,32

Âbsenzon. Therapie 127

der Lunge 147

Abtrelbung und Unfrucht- Adynamie bei Hyper barkeit 261

ACC 76 bei Neugeborenen-Arzte, beamtete und Beblutung 799

Achillessehnenreflex und 9 in Krankenhäusern 669 Niéréninsuffizienz $\mathbf{7 6 3}$

- bei Schilddrúsenerkrankungen 101

Achiliessehneñiß 611

Ácidose, chronische 602

- renal-tubuläre 604

Affen-Malaria 1003

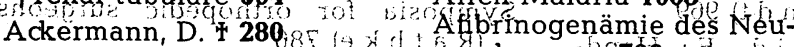

ACTHi undrifypierthyreose

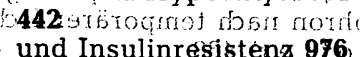

- und Insulinresistens

geboreneñ 793

Áfibrinogenamies 269 bu

Zahl 1964 285

Athylendiamintetraessig. saure und $d_{1}$ ierensteine 1020

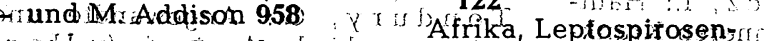

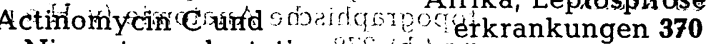
Nierentransplantation $\$ \&$ ig Aàmaglgbulinämie $830^{*}$

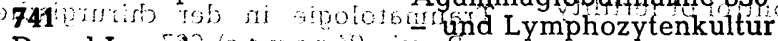

- D und Lymphozytens I 7$)$ aixolfi3

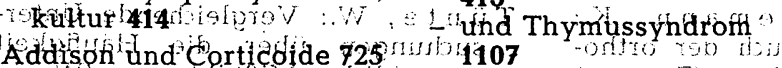

Aka ademie ider; Naturfor-

,scher Leopoldina, Mit-p? gliedswahl, 921

Akineton bei Parkinson

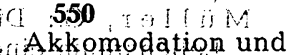
Fixation 1064

Akromegalie und Insulinresistenź 973

Aktinomy kóse der Lunge 712

ALD und Pleurodynie 1182

Aldoster on 284 \%

$\rightarrow$ und Antorexía nervosa

500ipolori.

- tbeil M. Addison 958

t und paraneoplastis cher

i Hypercorticismus $\mathbf{8 4 1}$

it und Renin 1077

- und Säure-BasenRegulation 602

Aldrich-Syndrom 830*

Alkalireserve und Reflexzeit 765

Alkohól und Kalkstoff veŕrgiftung 138

- Konsüm 141

- und feberzirrhose 684 $\mathbf{7 3 4}$ und Thiámin 236

Alkoholdehydrogenase und Bilirubin 877

Allergie durch Arzneimittel $727^{*}$

22 und Insulin 804

i durdh Medikamente $1071:$ : : is :

- und Nitrofurantoin 921

(5) und Penicillin 999

- und Zirrhose 685

Allergiediagnostik bei Asthma bronchiale 903

Alpen, Jodmangel 1202

Alterschírúgié und Durchblutungsstớrungen $\mathbf{8 1 2}$ ung $283^{*}$,

Al tesistenz 315

Alupent und Rezeptoren

blocker 146

Ambilhar 612

Amtenorthoe und Gefangenschaft 261 a

Amethopterin uñd Leukosen 245

Amine, biogene im Zentralnéryenśystem 318

$\varepsilon$-Aminocapronsäure in der Chirurgie 1206

7 und Gerinnung 317

Tund Prostatapperationen 297

Aminosauren und Entzündungsstoffe $\mathbf{\beta 6}$

Ammoniumbildung und Säure-Biasen-Regulation 601 is

Amniozentese bei Neur geborenenerythroblastose 229

Amöbenruhr in Europa 138

Amóbiasis, Chémor therapie 46

-, Leberabszeß und Szintigramm 820

Ampherion B und Cushing

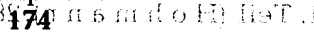

Amphotericin $B$ bei $\mathrm{My}^{-}$ kosen 713 , (i) $\rightarrow$ und Nierenyersagen 1037

Ampicillin Spiegelin Serum und Harn 206

Amputation und Becken-" arterien-Rekonstruktion 23

- bei Durchblutungsstörung 865

t Die fettgedruckten Zahleh bedeúten Originalarbeiten, die mit * versehenen Buchbesprechungen. $R=$ Arzt únd Recht.? 
Amtsarzt und Dienstaufsicht $365 \mathbf{R}$ ? ?

- undDienistvergehen $366 \mathbf{R}$..

Amylase, Stabilität 852

$\alpha$-Amylasebestimmung bei Pankreasdiagnostik 377

Anabolica und Osteoporose 1082 085

Anacidität histaminrefraktäre 282

Anämie, aplastische! 1064

-, - bei Thymus-Syndrom 1106

- und Enzephàlopathie 673

- nach fetomaternaler Passage 129

- hämolytische 473

- - und Enzephatopathie 675 of: hypochrome bej Kindern 1162

- nach Mágentesektion 846

Gottschaik, A., Túbingen 1215

- und Nephritis chronica 1091

Anästhesisten als $\mathrm{K}$ asssènärzte $608 \mathbf{R}$

Analeptika únd'Schlafmittelvergiftútigen 514

Analyse, pathologischchemische 1099*

Anatomie, topographische und angewandte 378 t

Anfälle, epileptische und therapie 126

Angina pectoris, Chirargie 641,1210 ;

- und Gichtanfall-............. äquivalehit 1171

- - und Nicotinsäure 725

_ - Stufenbelästungstest 890

Angiogramm bei Hirnvenenthrombose $\mathbf{1 0 2 8}$

Angiographie bei Beckenarterienverschlüssen $\mathbf{8 9 4}$

- bei Durchblutungsstörungen $\mathbf{8 1 2}$

- der Hirngefäße 663

- und spinale Angiome 173

- bei spinaler Apoplexie 929

- zerebrale 726*, 1053

Angiohämophilie 272

Angiokeratoma corporis

diffusum universale 241

Angiologie, Gesellschaft 237

Angiome, spinale und

Diagnostik 173

- vertebrale 925

Angiotensin und Renin 1077

Anorexia nervosa, Laborbefunde 499

Antibiotika und Bronchialinfektion 85

- bei Durchblutungsstörungen $\mathbf{8 6 6}$

- und Chirurgie 622

Antifibrinolytika $\mathbf{8 1 7}$

- und Prostataoperationen 297

Antigen und Antikörper 1197

- und Insulin 815

- und Lymphozyten $\mathbf{3 6 1}$
Antigene' und Lymphozy tenkultur 414

- und Nephritis $\mathbf{5 5 1}$,

- unidi Nierentransplantation 738

Antikoagulantien und : ; Chifurgie: 623

- und Hèrzinfarkt 582

- bei Herźinfarkt, IKG 978

- und Tetrachốrkohlenstoff 669

Antikörper und Anämien 173 ! i :

- und Diabetes 692

- Entstehung 197

- und Glomerulonephritis 551

- und Insulitin 315, 854

- und Masernimpfstoff 577

- und M. Werlhof 1071

- gegen Mycoplásmá pneumoniae 1014

- und:Schilddrüsenerktẩkưngen 438

- und Thyrivis 89

Antikonzipentien und Gellbsuckt 728 i

- und Léberfunktion 381

- ophithalmbiogisctie Störungen 469

Antimétaboliten bei Zirrhose 686

Antistreptoly sintitet und Polyarthritis 220

-, unspezifische 282

Antrum-Pylortsdys funktion 653 \&5 is

Anwaltskosten in der is li Sozialgerichttsbarkeit! 509 R

Aorta und Koronardurchblutung 1

Aorteninsuffizienz Chirurgie 1210

Aortenklappenersatz 1210

Aortenpunktion zur Angiographie $\mathbf{8 9 5}$

Aortenstenose, Chururgie 1210

- Korrektur 8

- supravalvuläre und Hypercalcämie $\mathbf{8 8 2}$

Aortographie und spinale Angiographie $\mathbf{1 7 3}$

Apfelsinen und Ileus 305

Apoplexie, spinale $\mathbf{9 2 5}$

Apothekenzwang und Arzneimittel $608 \mathbf{R}$

Aquocobalamin und Nierenclearance 1101

Aramin, Biosynthese 199

Arbeitsrecht 727*

Arcus senilis und Atherosklerose 43

Arfonad und Fettsäurenmobilisation 196

Armschmerzen, nächtliche 327

Arnold-Chiari-Mißbildung 801

Aronson-Stiftung 613

Arrythmie und Defibrillation 39

Arsenbehandlung in der Dermatologie 909

Arsenmelanose $\mathbf{9 0 9}$

Artane bei Parkinson $\mathbf{5 5 0}$

Arteria femoralis und Beckenangiographie $\mathbf{8 9 5}$

- - und Beckenarterien

Rekonstruktion $\mathbf{2 3}$
Arterien, Gefäßverschlüsse 1207 is Arteriosklerose u. Diabetes 698

- und Herzinfarkt 704

- und Laboratoriumsuntersuchungen 861 ind is

Arthritis, rheumatische

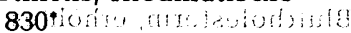

Arzneibehandlung,

Táschenbuch $\$ 16$ :

Arzneimittel :und Apo-

thekenzwang 608 R

-, Bêgriff $\mathbf{5 0 9} \mathbf{R}$

- und Porphyrie 959

und Thrombozytopetiie

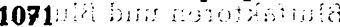

Arznefmittel-Allergien $727^{*}$

Arzneimittelforschung Fortschitte 46,

Arzneimittelgệsetz

Ergänzung:668: y-5:

Arzneispezílytäten,

rregistrierte 613 mat:

Arzt: àngestellte und Rentenyersicherung $231 \mathrm{R}$

- und Befundweitergabe 19 $1056 \mathrm{R}$

- undiGrundstükkserwerb $464 \mathrm{R}$

- Handbuch 469

- und Héilpraktiker 869 R

- und Hilfeleistungspflicht 1212

- vind Meldepflicht bei Fahruntüchtigkeit $41 \mathrm{R}$

- und Sorgepflicht 1058 R

- und Telefonabstellung $826 \mathrm{R}$

- am Unfallort 564*

- und Züchtigung $963 \mathrm{R}$

Arztbefunde, Weitergabe $1056 \mathrm{R}$

Arzthaftpflicht und Antitetanusinjektion $\mathbf{5 5 8}$ R

mit Estil 665 R

Ascorbinsäure und Kohlenhydratstoffwechsel $\mathbf{1 0 6}$

Aspergillom $\mathbf{7 1 1}$

Aspergillose der Lunge $\mathbf{7 1 1}$

Aspergillus-Pilze $1173^{\circ}$

Assistentenordnung in Rheinland-Pfalz $781 \mathrm{R}$

Asthma bronchiale, Allergiediagnostik 902

- - und Rezeptorenblocker 145

- und Emphysem, Therapie 82

Astrozytom, Nachweis 455

Atemminutenvolumen $\mathbf{6 6 0}$

Atemnotsyndrom und Blutungsübel 796

Atherosklerose, Erfahrungen 612*

- und Gliedmaßenarterien 863

Atlas der HNO-Krankheiten $327^{*}$

Atmung und Sport $1212^{*}$

- und Synchronisation 610

Atmungsforschung, Gesellschaftsgründung 877

Atom, Sicherheit und Rechtsordnung 1136*

Atosil und Liquordruck 772

ATP und Muskeldystrophie 111

Atropin und Parkinson 550

Aturban beil Parkinsonj550

${ }^{198} \mathrm{Au}$ und Hypophysen adenome 177 i:

Audiogramm bei 00 Dystrophia mýtotoniaas 947

Aufklärung bei Kropf-l operationen $608 \mathbf{R}$

Auge-und Hyperthyfeose: 1147 sise insilat

- unid Mydriaticum 666 : $9 \mathrm{~d}$

- und Phenothiazine 517

-, Tonometrie: 829: in

Augeh Säureverätzung 969

Augenfibel 1062 ? ?

Augenhintergrunds $51,1,09$ befunde bei Diàbetes und Fructosetherapie $\mathbf{3 0}$

Augeninnendrưck inna si Cortisontherapie 966

- Messung 829

Augenpigmentierungen und Chlórpromazin $833^{\circ}$

Augenprophylaxe nacho Credé 467 cioped

Augensdaluß und Filektrorenzephalogramm $\mathbf{5 3 9}$

Auttoantikörper bei i n Myasthenia gravis 396

- bei Schilddrüsenerkrankungen 437

- bei Thymus-Syndrom 1106

Autogenes Training 379*

Autoimmunhämatologie 473

Azathioprin und Lymphozytenkultur $\mathbf{4 1 4}$

- und Nierentransplantation $\mathbf{7 4 1}$

- und Zirrhose 686

Azotämie bei Herzkranken 726

- und Serumkalium 764

B

Bakterien und Streptomycin 1152 
Benadryl bei Parkinson 551

Bengalrosa und Bromsulphalein 211

Bergbau und Nitrosegase 900

Bergmann-Plakette, Verleihung 97

Bergsteigen und Corticosteroide 873

Berufskrankheit, Feststellung $366 \mathbf{R}$

Berufsordnung des Facharztes und Uberschreitung $91 \mathrm{R}$

Berufungsgerichtsbarkeit, Gesetz 926 R

Besoldung beamteter Arzte $463 \mathrm{R}$

Besoldungsgesetz in Hamburg $781 \mathrm{R}$

Best, Ch., Ehrendoktor 1214

Bestellungsordnung für Arzte, Entwurf 381

Betazol und Magensaft 356

- und Magenschleimhaut 596

Betriebsausgabe und Kongreßreise $720 \mathrm{R}$

- und Verkehrsunfall 139

Bewußtseinsstörungen und EEG 227

Bicarbonat des Harn, Bestimmung 405

- im Urin 325

Biguanid, Depotform 939

Biguanide, Verträglichkeit 873

Bilharziose, neues Mittel 612

Bilirubin und Alkoholdehydrogenase 877

Bilirubinbestimmung nach Jendrassik 233, 235*

Billroth-Resektion und Ileus 305

Binotal und Nierenfunktion 206

Biochemie 1062*

-, Lehrbuch $920^{\circ}$

- der Nebennierenrindenhormone $515^{\circ}$

Biochemie, praktische $380^{*}$

Biologie und finales Denken 295

-, Grundlagenforschung 620

- , molekulare $379^{\circ}$

Biopsie der Magenschleimhaut 1134

Blase und Harnabflußstörungen des Kindes 932

- und Querschnittslähmung 1111

Blasendruckmessung Fernübertragung 14

Blasenkrebs und Zigarettenrauchen 565

Blatt- und PlasmaVererbung 715

Blei, Bestimmung 235

Blitzkrämpfe, Therapie 224

Blut und Anorexia nervosa 499

- und autoimmunhämatolytische Erkrankungen 473

-, Biochemie 1062*

-, Komplementforschung $47^{\circ}$
Blut und Lymphogranulomatose 526

- und Ovulationshemmer 187

Blutammoniak, Bestimmung $235^{\circ}$

Blutbildung nach Magenresektion $\mathbf{8 4 6}$

Blutcholesterin, erhöhtes 43

Blutdruck, Regelkreis 1166

- und Koronardurchblutung 2

- und Reninaktivität 1074

- und Rezeptorenblocker 146

Blutfaktoren und Blutungsübel des Neugeborenen 794

Blutgasanalyse, präoperativ 659

Blutgase und Säureausscheidung 407

Blutgerinnungsstörung und Prostatakarzinom 119

Blutgruppe, Erblichkeit 1101

Blutgruppen, Fortschritte $1006^{\circ}$

- und Phosphatase 507

Blutgruppenverteilung $48^{*}$

Blutgruppenverträglichkeit, Erkennung 666

Blutkörperchen und Autoimmunhämatologie 973

Blutkonserve, Anforderung 1171

Blutkrankheiten und Enzephalopathie 673

Blutplättchen und Neugeborene 793

-, Ubertragung 1072

Blutproben bei Polyarthritis chronica 220

Blutsenkung und Hyperlipidämie 1133

Blutserum und sympathikoadrenales System 193

Blutspende, Karenz nach Schludkimpfung 388

Bluttransfusion und Gerinnungsdefekte 272

- und Konservenanforderung 1171

Blutung, epidurale 1186

-, materno-fetale 130

-, postoperative 1205

und Ulkus 655

Blutungen, intrakranielle des Neugeborenen 797

- und Schookniere 150

Blutungsübel, perinatale 793

Blutuntersuchungen, Physiologie in Schweden 915

Blutverlust nach Prostataoperationen 297

Blutvolumen und Venendruckmessung 1211*

Blutzellenpassagen beim Kind, transplazentare 129

Blutzucker und Anorexia nervosa 500

- und Ascorbinsäure 106

- und Diabetes 689

- und Diabetesprobleme 745
Blutzuckerbestimmungsmethoden 95

Blutzuckerregulation, Regelkreisschema 1166

Bornholmsche Erkrankung 1182

Botanik, allgemein $726^{*}$

Bourneville-Pringlesche Phakomatose mit Situs inversus $\mathbf{4 8 8}$

Boyden-Test und Schilddrüse 438

Brachialgia paraesthetica nocturna 327

Brenzkatechinamine und Fettgewebe 193

Bromsulphaleintest modifiziert 283

- und Radio-Bengalrosatest 211

Bronchialinfektion und Antibiotika 85

Bronchialkarzinom und Herzmetastasen $\mathbf{5 9 4}$

- und Hypercorticismus 839

Bronchiolitis und Emphysem, Therapie 82

Bronchitis und Emphysem $876^{\circ}$

- des Kindes und RS-Virus 153

- und Rezeptorenblodker 145

- und RS-Virus 53

Bronchopneumonie, perinatal 274

Bronchospasmolyse, Medikamente 85

Bronchuskarzinom und

Lungenszintigraphie $\mathbf{3 3 3}$

- und Rauchen $\mathbf{7 8 8}$

Bürger-Preis 970

Buformin bei Diabetes 939

Bundesseuchengesetz 380*

Burkitt-Tumor 235*

Bursa Fabricii und Thymus 88

Busulfan bei Polycythaemia 1004

Butylbiguanid bei Diabetes 939

C

Cadmium und Spermiogenese 1064

- und Osteoporose 1080

- und Reflexzeit 765

Calciumausscheidung im Urin 326

Calciumgehalt im Skelett 60

Calciumoxalatsteine, Litholyse 1020

Calciumphosphat-Steine, Litholyse 1020

Candida und Pimaricin 251

Candidiase der Lunge 711

Carotisangiogramm bei Hirnvenenthrombose 1033

Carotis-Insuffizienz, Chirurgie 662

Carotiskörperchen, Tumoren 493

Carotisphlebogramm

normales 1053

Caselitz, Hamburg 232

Caudaverletzungen und

Therapie 1109

CEE-Virus in Süddeutschland 1141

Chefärzte, Abgabenpflicht $780 \mathrm{R}$

Chefarzt und Abgabenregelung $417 R$

- und freiberufliche Tätigkeit $664 \mathrm{R}$

Chemie, Grundriß 284*

- , klinische 424

-, - und Standardmethoden $235^{\circ}$

-, pathologisch-physiologische $564^{*}$

-, physiologische $1062^{*}$

Chemoarchitektonik des Gehirns 76

Chemolitholyse der Nierensteine 1019

Chinidin und Vorhofflimmern 41

Chininmercuribisulfat bei Geschlechtskrankheiten? 378

Chirurg und Krankenhausträger $365 \mathrm{R}$

Chirurgie, amerikanische und Geschichte $874^{*}$

- und Antibiotika 622

- und Antikoagulantien 623

-, Blutungen 1206

- der Colitis ulcerosa 1001

-, experimentelle 617

- - und Gesellschaft 1174

- Gesellschaft und Präsidentenwahl 877

-, Krankheiten des Fortschrittes 622

- und Lungenfunktion 658

- und Transplantation 623

- Traumatologie $667^{\circ}$

, Zwischenfälle 283*

Chloramphenicol und Knochenmarkschäden 329

- und Tetanustoxoid 788

Chloride im Urin 326

Chlormerodrin und SäureBasen-Haushalt 605

Chlorochin und Lymphozytenkultur 414

Chlorpromazin und Augen- 
Chromosomen und Leukämie 1055

- und Plasma 715

Chromosomenanomalien als Abortursache 1094

-, Diagnostik 125

Chymotrypsin, Bestimmung 1172

Citrate und Tetanie-Anfall 1004

Citronensäure im Urin 325

Claudicatio intermittens

864

- - Beckenarterien-

Rekonstruktion $\mathbf{2 4}$

Clearance bei Ampicillin 206

- und Aquocobalamin 1101

- und Hypertonie 532

- und Rezirkulationsperitonealdialyse $\mathbf{7 0 7}$

Clearancemethoden $1211^{\circ}$

Cochlearisschäden durch Streptomycin $\mathbf{1 1 5 9}$

Coffeinlösung bei Bilirubinbestimmung 233

Cogentin bei Parkinson 550

Colektomie bei Colitis ulcerosa 1002

Coli und Streptomycin 1153

Colitis bei Pankreasadenom 634

- ulcerosa, Chirurgie 1001

Collumkarzinom und 6-Phosphogluconat-Dehydrogenaseaktivität 345

Colonresektion, Folgekrankheiten $\mathbf{6 2 4}$

Coma hepaticum, Therapie 221

- - und Zirrhose 687

Commotio cerebri und EEG 226

- und Schleuderung 590

Computer in der Pathologie 1174

Conn-Syndrom und paraneoplastischer Hypercorticismus 841

Contergan, Entgiftung 290

Coombstest 667

- und autoimmunhämolytische Erkrankungen 473

Coopersches Gerät in der HNO-Heilkunde 1016

Corbasil, Biosynthese 199

Corticoide und Ikterus hämolyticus $\mathbf{4 7 5}$

Corticosteroide und Basedow-Struma 1207

- beim Ekzem 599

- bei Höhenkrankheit 873

- und Insulinresistenz 975

- und Keratomykosen 505

- und Lymphozytenkultur 414

- und Osteoporose 1081

- und Pemphigus 1093

- und Polyneuropathie 1195

- beim Schock 95

Corticotropin $\beta^{1-24}$ als Testsubstanz 934

Cortisol bei Karzinomerkrankungen $\mathbf{8 4 1}$
Cortison bei Pocken? 457

Cortisonderivate in Klinik und Praxis $46^{\circ}$

Cortisontherapie und Augeninnendruck 966

Coxsackievirus-

erkrankungen $\mathbf{1 1 8 2}$

CPK, Haltbarkeit 852

- und Pleurodynie 1182

${ }^{61} \mathrm{Cr}$ und Morbus Werlhof 1069

C-reaktives Protein und Polyarthritis 514

Crecelius-Seifertsche

Blutzuckerbestimmung 95

Cryptococcose $\mathbf{7 1 2}$

Cryptococcus und Pimaricin 252

${ }^{137} \mathrm{Cs}$-Strahlentherapie beim Kehlkopfkarzinom (339

Cushing-Syndrom und paraneoplastischer Hypercorticismus $\mathbf{8 4 1}$

- -, Synacthen-Test 936

- - Therapie $\mathbf{1 7 4}$

Cyanquecksilber bei Grippe 325

Cyclophosphamid und

Nierentransplantation 741

Cystathioninurie und Vitamin B 6189

Cystinosebehandlung diätetische 1124

Czerny-Preis 1965141

D

Dämmerzustand und EEG 224

Danis-Preis 237

Darm und Salmonellosen 804

Darmstaeder-Preis 97

Darmverschluß im Alter 952

Datenverarbeitung in der Pathologie 1174

DDS bei Lepra 608

Defibrillation und Vorhofflimmern 39

Defibrinierungs-Syndrom und Prostatakarzinom

Depot-Biguanid und Diabetes 939

Depression, endogene und Psychoanalyse 421

Dermatologie, Arsentherapie 909

- Einführung 1099

-, Handbuch $95^{\circ}$

- und innere Krankheiten $380^{\circ}$

Dermatomykosen, Therapie 411

Dermatomyositis, Serologie 220

Dermatophyten und Pimaricin 251

Dermatosen, entzündliche 1062"

Desoxyribonucleinsäure und Proteinaufbau 179
Desensibilisierung mit

Inhalationsantigenen 904

Desinfektion bei Hepatitis 466

Desoxyribonucleinsäure und Plastide $\mathbf{7 1 9}$

Deszensus und Ver-

folgungsspätschäden $\mathbf{2 6 2}$

Dextroversio cordis und Q-Zacke 17

Diabetes mellitus $187^{\circ}$

- - und Acidose 604

- , Begutachtung 694

-, Behandlung und Diät $1135^{\circ}$

- - Depot-Biguanid 939

- Fibel $467^{\circ}$

- - Insulinbedarf 854

- - und Insulinresistenz 315, 973

- - , juveniler $\mathbf{7 5 0}$

- - - - und Pathologie 778

- Kapillarwandverdickung? 1137

- - kindlicher 216

- labiler 689

- latenter und Erfassung 753

- - und Leberzirrhose 377

- und Lipodystrophien 1171

- - und Trauma 695

Diabetesprobleme in Klinik und Praxis $\mathbf{7 4 4}$

Diät bei Glomerulonephritis des Kindes 1202

Diätetik bei Herzinfarkt 703

Dialyse und Clearance 709

Diamox und Säure-BasenHaushalt 605

Diarrhoen nach Diphenylhydantoin 1061

Diastase nach Magenresektion 844

- im Serum und Urin 784

Diastasebestimmung 377

Diathese, hämorrhagische 269

,-- und Enzephalopathie

676

,-- und Mononucleose 400

- bei Prostatakarzinom 119

Dibenamin und Fettsäurenmobilisation 196

Dibenzylin und Fettsäuremobilisation 196

und Schock 43

Dibutil bei Parkinson 550

Dickdarmentfernung be Colitis ulcerosa $\mathbf{1 0 0 1}$

Digitalisverabreichung, präoperative 877

Dihydrostreptomycin Entgiftung 1150

Dimethylbiguanid, Verträglichkeit 873

Diphenylhydantoin bei Epilepsie 127

-, Magen-Darm-Beschwerden 1061

Diphtherie- und Masernimpfstoff 572, 575

Dithizon und Hirnanfärbung 77

Diurese und Hypertonie 532

- und Narkose 407

Diuretika und Dünndarmgeschwüre 131

- und Säure-BasenRegulation 605

Divertikel, paraurethrale 514

Dopa und Blutdruck 198

Dopamin im Zentralnervensystem 318

Doppelniere und Bourneville-Pringlesche Phakomatose $\mathbf{4 8 8}$

,,-- erkrankte 448

Doriden, Entgiftung 290

Drumsticks und Chromosomendiagnostik 126

DPT-Polio-Masern-Impfstoff 571, 575

Duchenne-Muskeldystrophie 112

Dünndarmgeschwüre und Kaliumsalze 131

Dünnschichtchromatographie 81

Dumping-Syndrom 844

Duodenalgeschwür und Blutung 991

Duodenalsaft, Zytologie 548

Duodenalulzera und Diphenylhydantoin 1061

Durchblutungsförderung 863

Durchblutungsstörungen und Alterschirurgie 812

- der Beine und Beckenarterien $\mathbf{2 3}$

Durchfall und Pankreasadenom 634

Dystrophia muscularis progressiva und Innenohr 947

- myotonica und Innenohrschwerhörigkeit 947

Dystrophie, agastrische nach Magenresektion 846

\section{$\mathbf{E}$}

Eaton agent und atypische Pneumonie 429

Echoenzephalographie bei epiduraler Blutung 1188

- bei Hirnvenenthrombose 1028

Edmonston-Stamm und Masernimpfung 372

Ehe und Kinderzahl 1214

Ehrlich-Preis 97 
Ekzem, Therapie $\mathbf{5 9 8}$

Elektroenzepharögrammi Diagnostik und offene Augen 539:

- und Epilepsie 128

- bei Frühgeborenen ùnà Könzeptiónsalter 921

- Eberblick 223

Elektrokardiographie

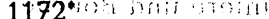

- im Kindesalter $187^{*}$

Elektronenmikróskopie 875* i $\$$ \$

Elektrokardiogramm bei Hérzinfarkt 978

- und-kammeranifangs gruppe'768

- und Q-Zadke15

- und Röntgen nologe $463 \mathrm{R}, 51 \hat{0} \div \mathrm{R}$

- beim Stuférbelástung's test $\mathbf{8 8 8}$ :! ?

- und Thromibolỳse dés Herzinfarktes $\mathbf{5 8 3}$

Elektrokárdiogriaphie, klinische $874^{*}$

Elektrolyte beiM. Addison 957

- und Reflexzeit 765

Elektrolythaushalt nach Operationen $\mathbf{6 2 2}$

Elektronenmikroskopie, Zellen und Gewebe 611

Elektrophorese und Léberdiagriostik 267

- und Lymphogranulomatose 527

- und Polyarthritîs 220

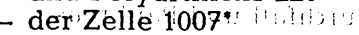

Elektroschweißgerät und Magenstörúngen 872

Ellbogen, Frakturen und Luxationen $234 \times$

Embolieverhutung und Kómpréssionsverband 73

Empfängniszeit, Unterschreitung 116

Emphysem und Q-Zacke 20

Emphysemforschung 876*

Encephales Syndrom nach: Schleuderung 591

Encephalitís congenita; Neuroanatonie $\mathbf{7 9}$

- zentraleuropäische in Süddeutschland 1441

Encephalopathie bei Blutkrankheiten 673

- und Lebercoma 221

Endokrinologie des Ovar 831*

Endoxan und Choriokarzinom 68

- und Nierentransplantation 741

Englisch für Mediziner $1137^{*}$

Enteritis und Lebensmittelbetriebe $134 \mathrm{R}$

-, Neuerkrankungen 1009

- Problematik $\mathbf{8 0 4}$

Enterokokken und Streptomycin 1153

Entgiftung von Pharmaka 290

Entlastungslaminektomie 1113

Entwicklungsphysiologie $1007^{*}$
Entzünduñǵ 920 :

Enitzündungsstoffe 33

Enuresis urreterica $\mathbf{4 5 0}$

Enzephalitis und Adeno virus 256

- und Elektroenzephałogramm 226

ctind Foctidruck 94 :

- und Mumps 282

Enzymaktivitätén und Fettstoffurechs

- und pléurodyniè 1182

Enzymbéstimmung im Blut 163

Enzymdiagnostik des Herzinfarktes 583

Enzyme 1099*

- und Blutungsúbél des Neugeborenén 795

- und Entgiftung 295,

- und Lipơgenése 1085

- Reaktiononen 286 ?

- und Seitenkettenthérie 1200

Enzyminduktion 180

Enzymsystemênd Erdálkaliiónen 96 :

Eosinophilentest bei Mr:i Addison 95

Ependymom, intrat medulläres 926

Epidermophytie, Therapie 412

Epiduralangiome 927

Epilepsie bei BournevillePringlesche Pharko'matose $\mathbf{4 9 1}$

- und Elektifoénzéphàlógramm 223, 540

trach Hirnvenenthrombose 1027

-, Liga und Informations schriften 236

- stereotaktische Eingriffe 984

-, Therapie $\mathbf{1 2 6}$

Erblichkeit der Blut gruppé 1101

Erbrectien ünd' Kardia beim Kind $\mathbf{3 0 8}$

Erbsche Muskeldystrophie und Therapie 111

Erbträger, plasmatischer 716

Erdalkali-Ionen $96^{*}$

Ermüdung 969*

Ernährung und Amenorrhoe $\mathbf{2 6 1}$

- des Kindes, Handbuch $1172^{*}$

Erysipel, Therapie 326, 821

Erythema chronicum migrans 233

Erythematodes und hämolytisches Syndrom $\mathbf{4 7 6}$

Erythroblastopenie bei Thymussyndrom 1106

Erythroblastose $1006^{*}$

-, Entwicklung 650

-, neue Wege der Therapie 229

Eyrthrozyten, feto-maternale Passage 129

Estil und Arzthaftpflicht $665 \mathrm{R}$

Euratom, Sicherheit und Rechtsordnung $1136^{\circ}$

Europa, Amöbenruhr 138

Euthanasie 236*
Euthyreose und Anti-

törper

(a)

- und Achillessehnenreflex 101

- und Hyperthy reose 1145

- und ${ }^{131} \mathrm{~J}$-Trijodthyronintest $\mathbf{5 4 2}$

Eversdiât und Multiple Sklerose 421

Evipan, Entgiftung 291

Exophthalmus und Hyperthyreose 1147

Experiment und Chirurgie 619 an Extràsystolen, Beurteilung 872

Exulcerátio simplex Dieulafoy 992

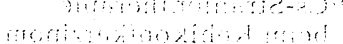

F

Fabry'Kranktieit'241"

Facharrzte und Notfall vertretung 366

Fáctiarzt únd Gericht 780 R

- und Notfalldienst $1170 \mathrm{R}$

- Pflichiten 91 R

Factiarztanerkénnung dúrch Gerichtsurteil 559 R !l ?

Facharztausbildúng und Rentenvérsicherung $230 \mathrm{R}$

Fachgebietüberschreitung. Schadenérsatz $91^{\prime} R$

Facialisparase durch Adenovirusinfektion 256

Faktor-V-Mangel 270

Faltenasymmetrie und Hüftgelenkluxation 172

Fahruntüchtigkeit und Meldepflicht des Arztes i.s.

Faktor VIII ưnd Hämotit philie 186

Felix-Haeffner-Preis 285.

Fermeñtaktivität; ; ; Regulation $1100^{*}$

- im Serum, Haltbarkeit 851

Fermente nach Magenresektion $\mathbf{8 4 4}$

Fermentforschung und Isoenzyme $612^{*}$

Fertilität, Beeinflussung $874^{*}$

- weibliche und Wiederherstellung 1047

Fettbestimmung im Stuhl 829

Fettembolie 1207

Fettgewebe, Stoffwechsel 773,1083

Fettleber und Farbstofftest 215

- und Schwangerschaft 1119

Fettsäuren bei Arteriosklerose 861

- und Heparin 1086

- Lipoproteidlipase 610

-, nichtveresterte und Diabetes $\mathbf{7 5 8}$

- und sympathiko-adrenales System 193

-, ungesättigte 470

- unveresterte und Regulation $\mathbf{1 0 8 3}$

Fetfsäurestoffiwechsel und Glucose $\mathbf{7 7 3}$

Fettsucht wiabetes $\mathbf{7 4 7}$

- únd Insulinresistenz 973 .

Fetus und Diabetes 216

Fibrinogenopenie, konstitutionelle 269

Fibrinolyse nach Próstataoperation 297

Fibroplasten für Chroniosomendiágnostik 125

Fibróblastenkultur 1097

Fibrose, retroperitoneale 97

Fiberskop zur Gastróskopie 1045

Fistel des Pankreas 644

Fixation und Akkomoda tion 1064 A

Fließbandhypothese der Allèrgìie 1197

Flüssigkeitstherapié, Praxisi $940^{\circ}$

Fluoreszenzthik roskopie und Amine im Zentral nervensystem 319

Folsăureantiagonisten bei Leukosen-245

Fornikotomie beil Epilepsie 985

Fortbildung und Sckiwéigepflicht $782 \mathrm{R}$

Fortschritt, chirurgischer und Krankheiten $\mathbf{6} \mathbf{2} \mathbf{2}$

Frakturen und Weichteilverletzungen $668^{*}$

Frakturenlehre, spezielle $234^{\circ}$

Frambösie 96

Frau und Bronchuskarzinom 788

Fremdkötpérzurücklassung bei Operation $463 \mathrm{R}$

Fruchtbarkeit, Beeinflus sung : $874^{*}$

Fructose und Hirndruck 770

- und Retinopathia dia bétíca 306

-, Wichtigkeit 467

Fructosediphosphat-Aldolase und Blutungsübel 795

Fructose-1,6-diphosphatase in Leber und Fett $\mathbf{7 7 4}$

Frühgeborene, Konzep- 
Gallenwege; Krankheiten nach Operation 624 !:

Gallenwégserkranküngen $1099:$

- und Lápatoskopie 1162

Gammaglobuline und Thÿmüs-Syndrom!1106

Gammasystem und Musketspannung:666.

Ganglien der Hand, Therapie 422

Ganglienzellveränderun-? gen nach Trabma 1126

Ganglion Gasseri und : i Trigeminusneuralgie $\mathbf{3 2 1}$

Gastrektomie, Folgen 624

- und Osteoporose 1081

Gastrin 653
Gastritis und Funktions:analyse 597

- haemorrhagica:991

- - Vagotomie 648

- nach Resektion 844

Gastroduoodenalưlkus; Vagotomie 648

Gastroenterologie: praktische 139

Gastroskopie, Indikationen und Grenzen 1045

Gastrotomie bei Exulceratio implex 993

Geburtenfolge bei Rh-Sensibilisierung 969

Gedächtnis, Pharmakologie 1060

- Untersuchungen $516^{*}$, i

Gedächtnisinhalte, Speicherung 728

Gefäßerkrankútigen; hypercalcämische $\mathbf{8 8 1}$

Gefäßleiden ind Diabetes 749

Géfảßoperationen im Becken 894

Gefäßschäden; diabetiscke 306

Gefä:Bverschlưsse arterielle und Chirurgie 1207 ics: 6

- und Beckenarterien-: Rekonstruktitom $\mathbf{2 3}$

Gefangenschafit : und / : ', ! : gynäkologische Spätt schảden:260

Gehtirn,Angiographie 726*

$\therefore$ und biogene Amine $\mathbf{3 1 8}$

- und Hypoglŷjkämie 1202

- Hypoxie 423 :

- Neuroanatomie 75 :

-, Verănderungen durch ? stumpfée Gewalt 1126:1

Gehirnfunktion 516 ; i a :

Gehirntümor und EEG 225

Geisteskrankheit und Einwilligung in Heiléingriffe $323 \mathrm{R}$

Gelbsehen 373 maisil

Gelbsucht und Antikonzipentien 728 ,

Gelenksteife 1208

Gemeinschaftspraxis, kassenârztlíche $780 \mathrm{R}$

Genaktivierung durch Hormone 179

Gene, Biosynthese 179

- und Gewebsantigene $\mathbf{7 3 8}$

Genetik, Fortschritte $830^{*}$

Genitalblutungen des $\mathrm{Neu}$ geborenen $\mathbf{7 9 7}$
Genftalerkrankingen $95^{*}$

Gentianaviolett ibei

Mykosen 714 10?

Geotrichose 712

Gericht und Facharzt 780 R

Gerichtsmedizin, Lehrbuch $1172 \%$

Gerichtsurteil über Fach alrztánerkennung $\mathbf{5 6 0} \mathbf{R}$

Gerinnungs defekte plas matische und Therapie

269,316 ?

Gerinnunigs aktoren und Neugeborene 794

Gerinnungsstörungen und Operation 12050rititry

Gerontologie| Fortschritte: $283^{*}$ is abs $:-6$

Geschlechtschromating Anomalien 126 \%

Geschlechtschromatinbefund, Abörtursache 1049 A

Geschlechtskrāñkheiten und Chinimercuribisulfat 378 :

-, Gesellschaft zur Bes B kämpfung 1009 ? ? nr?

- Handbuch 1062;

Gesellschaft, europäische : für experimèntelle Chirurgoie 1174 4

Gesellischaft für Innere: : i] Medizin, Präsidentenwahil 921

Gesichtsfeld und Rauchęn

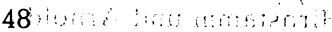

Gesichtsschädel Tumoren $46^{*}$

Gewebe, Fernstruktur 611*

Gewebezucht und Chromo somenanomalien $\mathbf{1 0 9 7}$ !

Gewebspunktion' bei Lungenprozesssen $\mathbf{3 7 6}$

Gewissen, Glaủbe und Ideologie 424

Gichtanfall, Aquiyalent und Angina pectoris 1171

Gierkesche:Glykogenose 453

Glatze, Altersformwandel $785^{\circ}$

Glaube und Gewissen 424*

Glaukom und Cortisoon-

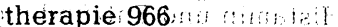

GLDH, Stabilität $\mathbf{8 5 3}$

Gliedmảenarterien, chronischer Verschlüß 863 :

Giloblastome, Nachweis

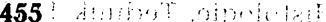

Gliose und Myelinisation 79

- und Hepatitis 165

Glissemént bei Hüft toof luxation 170 :

Glomerulonephritis und Acidose 603

- beim Kind, Diät 1202

-) Pathogenese 551

- und Reninaktivität 1075

- und Säure-BasenRegulation 603

- und Schockniere 149

Glomerulumfiltrat und Ampicillin 207

Glomerulusfiltration und Säure-Basen-Regulation 602

Glomustumoren, Differentialdiagnose 496
Glucocerebrosidase und

M. Gaucher 236

Glứcocorticoide und Autoimmunkrankheiten $\mathbf{4 7 8}$

- und Fettstoffwechsel 776

- und Leberkoma 222

- und M.Werlhof 1073

- und Leukosen 248

- und:Pilzinfektionen 505

- und Zirrhose 685

Glucocortidoidtherapie and Osophagusvarizen 466

Glucopháge, Verträglichkeit 87.3

Glucose and Hirndruck $\mathbf{7 7 0}$

- und Lipogenese 1084

- Mikrolitermethode $235^{4}$ :

-, Wichtigkeit 467 ,

Glucosebelastung und Fettsäuren $\mathbf{7 5 9}$

Glucosebelasturigen 754

Glucósedopipelbelástung: und Aporexia nervosa 500

Glucose-6-phosphatase in Fett und Leber 774

Glucose-6-phosphat $\mathrm{De}$ -

hydrogenase in Thrombo-

bytten 795

Glucosephosphat-Isomerase und Blutungsübèl 765

- - in Leber und Fett 774

Glucosestoffwechsel und Fettstof fwectisel 773

Glukagon und Lipolyse 776

Glutaminase: und SäureBasen-Regulation $\mathbf{6 0 2}$

Glutaminsäure und Géi dáchtnis 1060

Glutethimíd und Entgiftung 290

Gluthathion-Reduktase und Thrombozyten 795

Glycóaldehydphosphat-:

Dehydrogenase únd

Thrombozyten 795

Glykogenose Typ I (v. Gierke) 453

Glykogen phosphorylase in Leber und Fett $\mathbf{7 7 4}$

Gonadotropinand Ovulationshemmung $\mathbf{1 0 9 8}$

Gonadotropine und Anoréxía nervosa $\mathbf{5 0 0}$

Gonoblenorrhoeprophýlaxe 467

GOT, Haltbarkeit $\mathbf{8 5 1}$

- und Hepatitis 165

- und Pleurodynie 1182

GPT, Haltbarkeit 851

- und Pleurodynie 1182

Gravidität und Desensibilisierung 905

- und Lymphogranulomatose $\mathbf{5 2 2}$

- und Vigantolverabreichung $\mathbf{8 8 3}$

Grippeimpfungen 1098

Grippetherapie mit Cyanquecksilber 325

Griseofulvin bei Mykosen 412

Großstadt und Salmonellen $48^{*}$

Grundlagenforschung 832 *

Grundstücksverkauf an Arzte 464 R

Grundümsatz und Anorexia nerviosa 500

Schweden 915 i.:...?

- und Schilddrüsen-) ? $X$

erkrankungen 103 .

Gubaroffsche Falte und ir:

Kardiafunktion 309 s

Gürtelrose 263 :6t

Gutachtenhonorierung $721 \mathrm{R}$

Gutachterpflicht tund , I-

Schweigepflicht $1056 \mathrm{R}$.

Gynäkologie und Vertis

folgungsspätschäden 260 :

-, Zytodiagnostik 563:

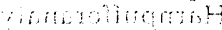

$$
\begin{aligned}
& \text { si) }
\end{aligned}
$$

$\mathbf{H}$

Härnagglutinationsreáktion nach Boyden 438

Hămangióme, kavernöse und Therapie 565

Hämatemesis $\mathbf{8 0 0}$

Hämatologie, Dífferentialdiagnose 667

- Normierung 141

Hämatome, epidurale

1186

- urnd EEG 226

Hämochromatose und Zirrhose $\mathbf{7 3 5}$

Hämodialyse und Rezirkulationsperitoneal. dialyse 706

Hämophilie A und B $\mathbf{2 6 9}$

- - und Faktor VIII 186.

- - Gesellschaft schweizerische 237

- des Neugéborenen 793

Haemophilus influenzae. Therapie 85

Hämorrhoidalleiden Injektion'stherapie 829

Hämorrholden und Pruritús âfर 45

Hämosiderose nach Eisen- 
Hanotsche Zirrhose 734

Harn, Barbitursäurenachweis 81

Harnabflußstörungen im Kindesalter 930

Harnammonium, Bestimmung 404

Harnanalyse, Methoden 403

Harndiastase 784

Harnkonzentrierung und Hypertonie 529

Harnleiter, Doppelbildung 450

Harnleiterstenose, subpelvine 931

Harnpufferanalyse und Säure-Basen-Haushalt 403

Harnsäure im Serum 921

- im Urin 325

Harnstoff und Ampicillin und Niere 209

- und Hirndruckerhöhung 771

- im Urin 325

Harnwege $1173^{\circ}$

Hassalsche Körperchen, Phagozytenfunktion 189

Haut und Arsentherapie 910

- und Fabry-Krankheit 241

- und Hyperthyreose 1147

- und innere Krankheiten $380^{\circ}$

- und Penicillin 999

Hautblutungen bei Prostatakarzinom 119

Hautdurchblutungsstörungen 865

Hautfett nach Magenresektion 846

Hautkrankheiten, Handbuch $1062^{\circ}$

Hautleistenvererbung $969^{*}$

Hautpilze, Therapie $\mathbf{4 1 1}$

Hautreaktion beim

Asthma bronchiale 903

Hauttransplantation und Lymphozytenkultur $\mathbf{4 1 5}$

HCG-Antiserum und Choriokarzinom 7

Hefen und Pimaricin 25

Heidelberg, PathologieLehrstühle 921

Heilberufsgerichte, Verfassungsmäßigkeit $509 \mathrm{R}$

Heileingriff bei Psychosen $323 \mathrm{R}$

Heilkunde und Optiker $720 \mathrm{R}$

Heilpraktiker und Arzt 869 R

Heilpraktikergesetz und kosmetische Eingriffe $558 \mathrm{R}$

- und Warzenbehandlung 869 R

Helfergin und Liquordruck 772

Henarin und Fettsäuren 1086

Hepatitis und Adenosindesaminase 163

- chronische 1210

- und Farbstofftest 213

- und Glucocorticoidtherapie 466
Hepatitis und Laparoskopie 1161

- und Leberzirrhose $\mathbf{7 3 3}$

- durch Leptospiren 371

- und Schwangerschaft 1114

-, Serumlabilitätsproben 267

-, Wohnungsentseuchung 466

- und Zirrhose 684

Hepatomegalie und Laparoskopie $\mathbf{1 1 6 1}$

Hepatosplenomegalie und Nierentransplantation 743

Herpes simplex bei Neugeborenen 1183

- zoster, Klinik und Therapie 263, 998

Herz und Abnabelungszeitpunkt beim Kind $\mathbf{4 5 9}$

- und Aortenklappenersatz 1210

- und Fabry-Krankheit 244

- und Koronardurchblutung 1

- und Hyperthyreose 1146

-, Physiologie in Schweden 914

- und QRS-Komplex 768

- und sympathikoadrenales System 193

Herzchirurgie der Aortenstenose 8

-, Folgekrankheiten $\mathbf{6 2 5}$

-, Sinus-venosus-Defekte 630

Herzerkrankungen, hypercalcämische $\mathbf{8 8 1}$

und Schockniere 149

Herzgeschwülste, metastatische 593

Herzhypertrophie und EKG 770

Herzinfarkt, Chirurgie 642

- Diätetik 703

- und PostmyokardinfarktSyndrom 1040

- und Q-Zacke 21

Herzinfarkt, Thrombolyse und EKG 978

- und thrombolytische Therapie $\mathbf{5 8 1}$

Herzinsuffizienz nach Ileus 954

- und Säure-Basen-Regulation 604

Herzkatheterisierung $516^{*}$

Herzklappenprothese bei Aortenstenose 13

Herzkranke, ABC 379*

-, Niereninsuffizienz bei Diurese 726

Herzkrankheiten, Kletterstufe zur Prüfung $\mathbf{8 8 4}$

Herzlage und Q-Zacke 16

Herz-Lungen-Maschine bei Aortenstenose 14

Herzruptur und Antikoagulantien $\mathbf{5 8 4}$

Herzschlag und Synchronisation 610

Herzstillstand bei Aortenstenosenoperation $\mathbf{1 2}$

Herzveränderung bei Wirbelsäulendeformierung 138

Herzvolumenbestimmung 327
Herzwiederbelebung 30

Herzzeitvolumen und Schock 1043

Hexobarbital, Entgiftung 292

Hexokinase und Neugeborenen-Thrombozyten $\mathbf{7 9 5}$

Hexokinasetest und Blutglucosewerte $\mathbf{7 4 5}$

${ }^{197} \mathrm{Hg}$-Chlormerodrin zur Hirntumordiagnostik 455

Hilfeleistungspflicht und Arzt 1212

Hilgenreinersches Einrenkungsphänomen $\mathbf{1 7 0}$

Hinterwandinfarkt und Q-Zacke 22

- und Thrombolyse $\mathbf{5 8 5}$

_ _ _ EKG 982

Hirnblutungen des Neugeborenen 797

Hirndruck, erhöhter und Therapie 770

- und Liquor $\mathbf{8 6 8}$

Hirndurchblutung bei Anämie 674

Hirngefäßerkrankungen und EEG 225

Hirnhautentzündung, Statistik 1009

Hirn-Hypoxie $423^{*}$

Hirnmetastasen, Nachweis 455

Hirnschädigung und Hypoglykämie 1202

Hirnstamm und ArnoldChiari-Syndrom 802

Hirnstrombild und Epilepsie 223

Hirntrauma und Blutung 1186

- und Elektroenzephalogramm 226

Hirntumor, RadioisotopenNachweis 454

Hirnvenenthrombosen $328^{\circ}$

-, innere und Diagnostik 1025

- Nosologie 1049

Hissscher Winkel und Kardiafunktion 309

Histalog und Magenschleimhaut $\mathbf{5 9 6}$

- und Magensekretion 356

Histamin und Entzündungsstoffe 33

Histokompatibilitätsgene 738

Histokompatibilitätstest $u$. Lymphozytenkultur $\mathbf{4 1 4}$

Histologie, Technik $1135^{\circ}$

HNO und Streptomycintherapie $\mathbf{1 1 5 8}$

Hochdruck, ABC 920

-, portaler 1207

Hochdruckforschung 563*

Höhenkrankheit und Corticosteroide 873

Hörstörung bei Dystrophia myotonica 947

- und Streptomycin 1155

Hoffmann-Gesellschaft, Präsident 788

Hohlvenen, Umpflanzung 632

Holzbock, Therapie 186

Homburg-Preis 470

Hormonanalysen bei M. Addison 958

Hormone, anabole und

Stimmstörungen $\mathbf{5 5 5}$

- und Diabetes 690

- und Genaktivierung 179

- und Koronardurchblutung

- und Lipogenase 775

- und Lipolyse 1084

- zur Ovulationshemmung $830^{\circ}$

- bei Potenzminderung 234

Hormonwirkung und Thymus 88

Hornhaut, Pilzinfektionen 505

Hospitalismus und Staphylokokken 425

Hüftgelenk, Luxationsfraktur 1208

Hüftgelenkskontrakturen und Nervus obturatorius 313

Hüftgelenksluxation, Frühdiagnose 168

Hufelandpreis 1965921

- 1966141

- 1967.1214

Human-Insulin, synthetisches 565

$-,-1213$

Hundespulwurm bei Kindern 849

Hyalin-Membran-Syndrom 822

Hydergin bei Schock $\mathbf{4 3}$

Hydralazin als Antivitamin $B_{6} 1137$

Hydrocortisonazetat beim Ekzem 599

Hydronephrose $\mathbf{4 5 0}$

Hydroureter 450

11-Hydroxycorticoide und Synacthen-Test 935

Hydroxylapatitgehalt der Wirbelsäule 61

5-Hydroxytryptamin im Zentralnervensystem $\mathbf{3 1 8}$

Hydrozephalus des Säuglings $\mathbf{8 0 1}$

Hyperaldosteronismus und Alkalose 604

Hyperbilirubinämie, 
Hypertonie, ABC 920*

-, essentielle und Harn-

konzentrierung $\mathbf{5 2 9}$

-, Forschung 563*

- und Niere 329

-, postenzephalitische 94

- und Reninaktivität $\mathbf{1 0 7 4}$

Hypertriglyceridämien, essentielle 27

Hypofibrinogenämie 269

- bei Prostatakarzinom 119

Hypoglykämie und Halbseitensyndrom 1202

- unter Silubin retard 943

Hypokaliämien 604

Hypopharynxkarzinom, Telecaesiumtherapie $\mathbf{3 3 9}$

Hypophyse und Blutzuckerregulation 1166

- und Diabetes 690

Hypophyse und Diabetes 690

- und Insulinresistenz 977

- und Lipolyse $\mathbf{7 7 6}$

Hypophysenadenome, Therapie $\mathbf{1 7 7}$

Hypothyreose und Achillessehnenreflex 101

- und ${ }^{131} \mathrm{~J}$-Trijodthyronintest $\mathbf{5 4 2}$

- und Niereninsuffizienz 767

- nach Radiojodtherapie 438

Hypotonie nach Operationen 622

- und Reninaktivität $\mathbf{1 0 7 4}$

Hypoxie, zerebrale $423^{*}$

I

Ideologie, Glaube und Gewissen $424^{\circ}$

Ikterus und Gelbsehen $\mathbf{3 7 4}$

-, hämolytischer 475

-, - und Kriterien 918

- neonatorum und $\mathrm{Ab}$ nabelung $\mathbf{4 6 0}$

$\rightarrow$ postoperativer 625

- als Spätgestose $\mathbf{1 1 1 4}$

Ileus im Alter 952

- bei Dünndarmgeschwüren durch Kalium 132

- nach Magenresektion 303

-, postoperativer $\mathbf{4 5 3}$

Imido und Magenschleimhaut $\mathbf{3 5 6}$

Immunadhärenz und Insulinallergie $\mathbf{8 1 5}$

Immunbiologie und Thymus $47^{*}$

Immunfluoreszenz und Insulinallergie $\mathbf{8 1 7}$

Immunitätssystem, thymoide Organe 87

Immunkörper und Choriokarzinomzellen $\mathbf{6 6}$

Immunologie und Insulin 858

- und Komplementforschung $47^{*}$

- und Nierentransplantation $\mathbf{7 3 8}$

- und Thymus 1108

Immunozyten in der Gravidität 130
Immunreaktionen 920* Immunsuppressiva $\mathbf{7 4 1}$

- von Thalidomid 1132

Immunthrombopenie $\mathbf{1 0 7 1}$

Immunthyreoiditis $\mathbf{4 3 7}$

Impfplan für Kinder 877

Impfung gegen Grippe 1098

Imuran $\mathbf{7 4 1}$

- und Lymphozytenkultur 414

Infarkt und Q-Zacke 21

- und Thrombolyse $\mathbf{5 8 1}$

Infarkte, rudimentäre $\mathbf{9 8 3}$

Infarkttherapie und EKG 979

Infektionskrankheiten in den Ländern der Bundesrepublik Deutschland (7. XI.-4. XII. 1965) 142 (5. XII. 65-1. I. 66) 330

2. I. -29. I. 66) 518

(30. I.-26. II. 66) 788

- (27. 2.-26. 3. 66) 1101

(27. III.-23. IV. 66) 1213

19651008

- und Lebensmittelbetriebe $133 \mathrm{R}$

Infertilität, weibliche $\mathbf{1 0 4 6}$

Infrarotthermographie in der Klinik 96

Immunglobulin, Nachweis 425

Innenohrschwerhörigkeit bei Dystrophia myotonica 947

Inselapparat und juveniler Diabetes $\mathbf{7 7 8}$

Inselzelladenom, seltenes 634

Insufflation bei Kreislaufstillstand $\mathbf{3 0}$

Insulin und Diabetes $\mathbf{7 4 7}$

-, humanes synthetisches 565,1213

-, Synthese 565

Insulinaktivität des Serums und Heparin 1086

Insulinallergie, Antigenbindung $\mathbf{8 1 4}$

Insulinresistenz $\mathbf{3 1 4}$

-, Klinik 973

-, Pathogenese 853

Intensivbehandlung 1210

Intern $727^{*}$

Intestinalblutung, akute 991

Intrakutantest bei Lungenmykose $\mathbf{7 1 3}$

Inulin-Clearance und Ampicillin 208

Invalidität bei multipler Sklerose 996

Irispigmentation und Mydriatica 285

Ischämie und Nephron $468^{*}$

Isoagglutinine, thrombozytäre 1073

Isocitrat-Dehydrogenase in Thrombozyten 795

Isoenzyme $612^{*}$

Isometrie $517^{*}$

Isoprenalin und Koronardurchblutung 3

Isthmusstenose und Korrektur, Folgekrankheiten 626

Ixodes rizinus, Therapie 186
$\mathbf{J}$

${ }^{131} \mathrm{~J}$-Diagnostik der Struma 1206

${ }^{131} \mathrm{~J}$-Eiweiß-Makroaggregat zur Lungenszintigraphie 333

${ }^{131} \mathrm{~J}$-Trijodthyronintest im Kropfgebiet Südbadens 541

Jendrassik'sche Bilirubinbestimmung 233

Jod bei Pilzen 412

Jod-Bengalrosa zum Leberszintigramm 820

Jodmangel in den Alpen 1202

\section{$\mathbf{K}$}

Kälteagglutination und Mycoplasma pneumo niae 1014

Kälteagglutinine und Blutkrankheiten $\mathbf{4 7 4}$

Kälteagglutininkrankheit und Enzephalopathie $\mathbf{6 7 5}$

Kälteanwendung bei HNOOperationen $\mathbf{1 0 1 6}$

Kältehämoglobinurie $\mathbf{4 7 7}$

Kälteschäden und SanarelliShwartzman-Phänomen 285

Kaffeebohne, Theobrominfund 49

Kahnbeinpseudarthrose und Unfallfolge 421

Kalium und Achillessehnenreflex $\mathbf{7 6 4}$

- und Säure-BasenRegulation 602

Kaliumausscheidung im Urin 325

Kaliumjodid bei Mykosen 714

- und Sekretolyse 85

Kaliumplasmawerte Therapie erhöhter $\mathbf{3 5 9}$

Kaliumsalze und Dünndarmgeschwüre 131

Kalkembolie nach Aortenstenosenoperationen $\mathbf{1 4}$

Kalkstickstoffvergiftung und Alkohol 138

Kallikrein und Entzündungsstoffe $\mathbf{3 4}$

Kallikrein-Inhibitor nach Prostataoperationen $\mathbf{2 9 7}$

Kaltkauterverfahren zur Warzenbehandlung $869 \mathrm{R}$

Kaltlichtendoskop 1160

Kammeranfangsgruppe im EKG 768

Kapillarwandverdickung beim Diabetes? 1137

Kardiafunktion beim Kind 308

Kardiakarzinom, Diagnostik mit Radiophosphor $\mathbf{9 0}$

Kardiatamponade 422

Karzinogenese, Statistik 135

Karzinom $785^{\circ}$

- und Herzmetastasen 593

- und Hypercorticismus 840

- der Lymphknoten Lymphographie 1178

Karzinom des Magens,

Fiberskop 1046

- und Radiophosphorspeicherung 90

- und Zigarettenrauchen 565

Karzinome, arsenbedingte 909

-, Kryochirurgie im HNOGebiet 1018

Kassenarzt und Anästhesisten $608 \mathrm{R}$

-, Gemeinschaftspraxis $780 \mathrm{R}$

- und Notfallvertretung $826 \mathrm{R}$

Kassenarztrecht $1100^{\circ}$

-, Streitfragen $465 \mathrm{R}$

Kassenarztsachen und Sozialrichter $\mathbf{5 5 8} \mathrm{R}$

Katheterisierung des Herzens $516^{\circ}$

Kehlkopfkrebs, Telecaesiumtherapie $\mathbf{3 3 9}$

Keimdrüsen und Diabetes 691

Keimling und Virus 1008

Kemadrin bei Parkinson 550

Kephalhämatom des Neugeborenen $\mathbf{7 9 7}$

Keratomykosen und

Corticosteroide $\mathbf{5 0 5}$

Kernphysik $234^{*}$

Ketoacidose und Insulinresistenz $\mathbf{3 1 4}$

Ketose-1-phosphatAldolase in Leber und Fettgewebe $\mathbf{7 7 4}$

17-Ketosteroide und Anorexia nervosa $\mathbf{5 0 0}$

- im Urin 326

Kind und Abnabelung 459

- und BCG-Impfung 479

- und Blutungsübel $\mathbf{7 9 7}$

-, Blutzellenpassagen, transplazentar $\mathbf{1 2 9}$

-, Cystinosetherapie $\mathbf{1 1 2 5}$

- und Diabetes 693, 75

_ _ - fetale Inselschädigung 216

- und Einwilligung in Heileingriffe $323 \mathrm{R}$

-, Eisenmangelanämie 1162

-, Elektroenzephalographie $187^{*}$

- und Epilepsietherapie 127

- und feto-maternale Passage 129

-, geistig behindertes und Bundesvereinigung Le- 
Kind und Masernschutzimpfung! 569,575

- Membransyndrom 423.

- und Myelinissationsgliọse 79 and

- und Naevuszellnaevus 1098:

- und Nierenvenenthrombose 57

- , Pädologie 786*:

- und Pneumonie perinatal 273

- und Respiratorysyncytial-Virus 53,153

- und Schwerhörigkeit bei Streptomycin 1155

- und Spulwurmbefall vor Tieren 848

-, Toxoplasmose 389

-, Wachistumsonsbeinflussung 1005

Kinderarzt, Notfallfibel $422^{*}$

Kinderchirurgie 1209

Kinderheilkunde, Handbuch $1172^{*}$

- Lehrbuch 563*;

Kinderkrankheiten; Therapie $235^{*}$

Kinderzahl und Ehe 1214

Kindesbeschneidung: $464 \mathrm{R}$

Kinine und Entzündungsstoffe: 34

Kininogenase 34

Klappensprengung be Mitralstenosen 436

Kleinhirn und Traumaveränderungen 1126

Kleinschmidt-Preis 788

Kletterstufe zur Leistungs prüfung 890

Klimakterium und Osteóporose 1081

Khìngel, abgestellte und Arzt 826 $\mathrm{R}$

Klinik und Chemie 424

Kniegelenkskontrakturen und $\mathrm{N}$ obturatorius $\mathbf{3 1 3}$

Knochen und Calciumgehält $60^{\circ}$

Knochenatrophie bei Osteoporose $\mathbf{1 0 8 0}$

Knochenmark zur Chromosomendiagnostik 125

Knochenmarkschäden nach Chloramphenicol 329

Knochenmarkstransfusion bei Anämie von Zwillingen 1064

Koagulopathien, hereditäre 269

Kochsalzdiurese und Hypertonie $\mathbf{5 3 2}$

Körpergewicht und Hyperthyreose 1146

- und Silubinretard bei Diabetes 940

Körperkrankheiten und Psyche 968.

Kohlenhydrate und Hypertriglyceridämie $\mathbf{2 8}$

- und Lipämie 703

Kohlenhydratstoffwechsel und Ascorbinsäure 106

- und Depot-Biguanide 939

- und Diabetes 689

- und Enzyme 774

- und Lipolyse 1085

Kohlensäurespannung und Săure-Basen-Regulation 602
Kohlenwasserstoffe in Lösungsmitteln, Toxikologie 668:

Kollagenkrankheiten, Serologie 220 - a

Koma und EEG: 227

Kommissurotomie bei Epilepsie 986

Komplementforschung 47 *

Komplementsystem und: Glomeruflonephritis : 552

Kompressionsfrakturen der Wirbeilsäule 1112

Kompressionsverband und Venenerkrankungen der Beine 73

Kongreßreise als Betriebsausgabe $720 \mathrm{R}$

Kontinenz, anale im

Kindesalter 1210

Kontrastmittel für Lymphsystem 1177

Kontrazeptiva undn Hyperkoagulabilität 1.87

Konzentrationslager und gynäkologische: Spätschäden $\mathbf{2 6 0}$

Konzeption und Ovulationshemmer 1134

Konzeptionsalter, Frühgeborener 921

Kopf und Schleudertrauma $\mathbf{5 8 9}$

Kopfhaar, Altersformwandel $785^{\circ}$.

Kopfschmerz und EEG 225

Koronardurchblutung. Regelung 1

-, Pharmakologie 727:

Koronargefäß chirurgie 642

Koronargefäßerkrankungen und Diät 704

Koronarreserve und Stufenbelastung: $\mathbf{8 8 4}$

Kontaktekzem $\mathbf{5 9 8}$

Kosmetik und Heilpraktikergesetz $\mathbf{5 5 8} \mathrm{R}$

Krankenanstalten, Selbstkostenrechnung $509 \mathrm{R}$

Krankenhaus und ArzteZahl 669

-, psychiatrisches und Sicherungen 416 R

Krankenhausarzt und Leichenschau $417 . \mathrm{R}$

Krankenhausinstitutsleistung und Ambulanz $720 \mathrm{R}$

Krankenhausröntgenologe und Umsatzsteuer $1169 \mathrm{R}$

Krankenhausträger und Chirurg 365 R

Krankheiten, meldepflichtige 1008

-, neuromuskuläre 1136*

- und Psyche $968^{\circ}$

Kreatin und Muskeldystrophie, Therapie $\mathbf{1 1 3}$

- im Urin 325

Krebs $785^{*}$

-, Chemotherapie $46^{*}$

- Statistik 328* 1211

Krebsentstehung aus Warzen $871 \mathrm{R}$

Krebsforschung, Fortischritte 1136 .

Krebsoperationen und Zytostatika 514, 121 :

Kreislauf, Kybernetik $1212^{*}$
-, Phy'siologie in Schweden 915

- undistympathiko-

à arenales System 193

Kreislauttforichung, Gesell scbáft und Präsidenten-

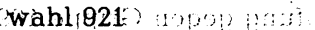

Kreislaufkranke, $\mathrm{ABC} 379^{\circ}$

Kreislaufstillstand, akuter 29

kuter
kutes

Kreislaufversagen $n_{r}$ akutes 1043

Kreuzprobe und Rhesus: untergruppe 186

Kreuzschmerzen und Multifidus-DreieckSyndrom 445

Kropf und Antikörper 439

- und ${ }^{181}$ J-Trijodthyronintest 541:

Kropfoperationen, Auf klärưngspflicht $608 \mathrm{R}$

Kryochirurgie im HNOFachgebiet 1018

Kündigungsschutzgesetz $1063^{*}$

Kunstfehler bei Kindes: beschnieidung: 464 $R$

Kuru-Krankheit :831*

Kurzwellentherapiegerät, Störungén 967

Kybernetik $1212^{*}$

- in der Médizin 116

Kyphosen, juvenile 44

$\mathbf{L}$

in:
30

Laboratorium und Normalwerte 1061

Laboratoriumsbefunde bei Herpes simplex des Neugeborenen 1184

Laboratoriumsdiagnostik. der Nebennierenrindeninsuffizziènz 957

Laboratoriumsuntersuchungen bei Arteriosklerose 861

Laboratory Animals, Committee :1009

Lactatdehydrogenase im Fettgewebe und Leber 774

- in Thrombozyten $\mathbf{7 9 5}$

Längenwachstum, Beeinflussung 1005

Lärmbekämpfung und Medizin 1101

Laevadosin bei Muskeldystrophie 111

Laevosan und Hirndruck 770

Laminektomie bei Rückenmarksverletzungen 1111

Langenbeck-Preis 877

Langerhanssche Inseln. Pathologie $\mathbf{7 7 8}$

Lapäróskopie und Leberdiăgnostik 1160

Larynxkarzinom, Telecaesium therapie $\mathbf{3 3 9}$

Latex-Fixationstest 220

LDH, Háltbarkeit 851

Leben, Euthanasie 236

Lebendimpfung gegen Másern 572,575

Lebensmittelbetriebe und Infektionskrankheiten $133 \mathrm{R}$

Lebensmittelbetriebe und Salmoniellen-Erkrankungen 808

Lebensversicherung :und Gutachtenkonorierung 721 R

Leberi und CoxsackieViren 1182

- Diagnostik mit Radioisotopen 819

- Und Fairbstoffteste $\mathbf{2 1 1}$

- und Gerimnung 316

- und Ikterus in der Sohwangerschaft 1119

- und Lyamphogranulomatose 524:

- nàch Magenresektion 845

- und Medikamente $188^{\circ}$

- urd IPharmaka 290

- und Porphyrie:959.

- und Serümlabilitäts proben 267

Lēberabszeß, Szintigramm 820 of

Leberbiopsie: nach Menghini 1160 ? ?

Leberdiagnostik und Laparoskopie 1160

Lebererkrankunğen $1099^{*}$

- und Adenosindesaminase 163 :

- und Arzneimittelentgiftung 293

- und Elektroenzephalogramm 227

Leberfermente und Glucocorticoide 1100 ?

Leberflecke und Heilpraktikerbehandlung $871 \mathrm{R}$

Leberfunktion und Antikonzipientien 381

Leberfunktionsproben, Suchtest 378

Leberkoma, Therapie 221

Leberschäden, posthepatische und Kriterien 9.18 :

Leberschutztherapie $\mathbf{6 8 5}$ 
Lendenwirbelsäule Multifidus-DreieckSyndrom 447

Leopoldina, Naturforscher und Mitgliedswahl 329

Lepraforschung, Probleme 606

Leptospirosen in Nordafrika, Ursache $\mathbf{3 7 0}$

Lese-RechtschreibeSchwäche, Diagnostik und Therapie 461

Leukämie, myeloische 1055

- in Polycythaemia vera 56

- und Thymus $47^{*}$

Leukämieforschung $235^{*}$;

Leukoenzephalopathie, multifakale 679

Leukosen, Enzephalopathie 678

- im Kindesalter, Methotrexat-Therapie 245

Leukozyten und Insulinallergie $\mathbf{8 1 4}$

- und Lymphogranulo matose $\mathbf{5 2 6}$

-, materno-fetale Passage 130

Leukozytenemigration und Entzündungsstoffe $\mathbf{3 7}$

Leukozytenkultur zur Chromosomendiagnostik 125

Leukozytenphosphatase und Leukämie 1055

LE-Zellen bei Polyarthritis 219

Libidominderung, Therapie 234

Liljestrand, G., 80. Geburtstag 1059

"limb girdle." Muskeldystrophie 109

Linkshänder in der Schule 783

Linsen in der Cystinosetherapie 1125

Linsentrübung und Phenothiazine 51.7

Lipämie und Blutsenkung 1133

- und Kohlenhydrate $\mathbf{7 0 3}$

Lipase nach Magenresektion $\mathbf{8 4 4}$

Lipodystrophien beim Diabetes 1:17:1

Lipogenese und Hormone 775

- und Lipolyse 1083

Lipoide und Arteriosklerose $\mathbf{8 6 2}$

Lipoidnephrose und Glomerulonephritis 348

Lipoproteidlipase Wirkung 610

Lipoproteine und Hypertriglyceridämie $\mathbf{2 7}$

Liquor und epidurale Blutung 1187

Liquordrucksenkung $\mathbf{7 7 1}$

Liquordynamik $\mathbf{8 6 7}$

Liquorpassage bei Rückenmarkverletzung 1110

Litholyse bei Nierensteinen 1019

Lobelin- und Nicotin Entwöhnung 233

Lösungsmittel, Toxikologie $668^{*}$

London, Pestjahr 1665 $1063^{\circ}$
Lues connata: 96

- , Therapie $96^{\circ}$

Luftwege und RS-Virus $\mathbf{5 3}$

Luftwegsinfektionen mit Mycoplasmaipneumoniae 1.014

Lumbalpunktion bei Hirn venenthrombose 1028

Luminal, Entgiftung: 291

- bei Epilepsie 127

Lunge, Atmung und Sport $1212^{*}$

- bronchitisches Syndrom 40

- und Gewebspunktion 376

- und Hyalin-MembranSyndrom 822

- und Nitrosegase 899

- Pneumonie perinatal 273

- und RS-Viren 53, 153

- und Säure-Basen-Haushalt 405, 600

Lungenblutung des Neugeborenen 799

Lungenchirurgie und Folgekrankheiten 625

Lungenemphysem und Acidose 604

-, Therapie $\mathbf{8 2}$

Lungenerkrankungen Röntgenbild $235^{\circ}$

- , unspezifische $876^{\circ}$

Lungenforschung, Gesellschaftsgründung 877

Lungenfunktion und Pneumotachographen 51.4

-, Prüfung in Schweden 915

- Prüfung: in der Praxis 725

Lungenfunktions diagnostik, präoperative 658

Lungenkrankheiten und Rezeptorenblocker 145

Lungenkrebs und Lungenszintigraphie $\mathbf{3 3 3}$

Lungenmykose, Diagnose 711

Lungenödem, Procain therapie 1202

Lungenszintigraphie bei Lungenkrebs $\mathbf{3 3 3}$

Lungentuberkulose und BCG-Impfung 479 .

Lungenveränderungen und Wirbelsäule; 138

Lupus ery thematodes $830^{*}$

- Serologie 220

Luxatio coxae, Diagnostik 168

Luxationsfraktur im Hüftgelenk 1208

Luxationslehre, spezielle $234^{*}$

Lymphangioadenographie 1177

Lymphdrüsen und Lymphogranulomatose 523

Lymphknotentumoren Diagnostik 1178

Lymphogranulomatose $\mathbf{5 2 1}$

- und Enzephalopathie 679

- und Gravidität 522

Lymphogranulomatose und Lymphographie 1178

- und Tuberkulose 528

Lymphopathia venerea $96^{*}$

Lymphosarkom und Lymphögraphie 1178

Lymphozyten und Lymphogranulomatose $\mathbf{5 2 6}$
Lymphozyten und Thymus 87

Lymphozy tenkultur 360

-, Bedeutung 413

Lymphozyten-TransferTest 739

Lymphsystem, retroperitoneales und Lymphangioadenographie 1177

LyovactRubeovax, Schutzimpfungen $\mathbf{5 6 9}, 575$

Ly:søsomen und Entzündungsstoffe 35

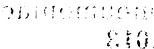

$$
\begin{aligned}
& 465
\end{aligned}
$$

M

Magen und Anorexia nervasa 499

- und Fiberskop 1045

- des Kindes und Kardiafunktion 310

Magenausheberung nach Lambling 650

Magenblutung und Schockniere 1449

- und Tamponade 422

Magenchirurgie 649

- und Nachkrankheiten 624

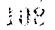

Magengeschwür und Blutung 991

Magenkarzinom und Blutgruppe 49

- und RadiophosphorDiagnose $\mathbf{9 0}$

Magenkrankheit und Elektroschweißgerät. 872

Magenkrebs, Therapieerfolge 336

Magenresektion und Funktionsanalyse $\mathbf{5 9 7}$

- und Ileus 303

- und Magenkarzinom 336

-, Störungen 843

Magensaugbiopsie, automatisch gesteuerte 1134

Magenschleimhaut Funktionsanalysen 596

- Sekretionsanalysen 356

Magenspülung bei Vergifteten $\mathbf{4 8 5}$

Magenulcus; dissezierendes 1121

Magnesium, Bestimmung $235^{\circ}$

- im Urin 326

Makroglobulinämie Waldenström und Enzephalopathie 676

Malaria eine Zoonose? 1003

Malat-Dehydrogenase und Thrombozyten 795

Mandeloperation und Züchtigung $963 \mathrm{R}$

Mangeldurchblutung zerebrale und Chirurgie 662

Mann und Bronchuskarzinom 788

Mannit und Hirndruckerhöhung 771

Mannitdiurese $\mathbf{4 0 7}$

- hypertonische 532

Marboran bei Pocken $\mathbf{4 5 6}$

Martini-Preis 1966, Verleihung 470

Martini-Stiftung 833

Masernschutzimpfung aktive 569,575

Masson-Tumoren und Glomustumosen 496

Mastdarmlähmung 1111

Mastodynie, Therapie 1005

Mastzellen $140^{\circ}$

Matrizenhypothese der Seitenketten theorie $\mathbf{1 1 9 7}$

Mauriac-Syndrom 691

Max-Planck Gesellschaft

Berufungen 189

Mediastinalprozesse und Gewebspunktion 376

Medikament und Thrombozytopenie 1071

Medizin, forensische 1172

- klinische und Grund-

lagenforschung 832

- und Neuroanatomie 75

$\rightarrow$ Regulation und Kyber netik 1164

- im Sozialrecht 876*

Mediziner, Englisch 1137

Medizinschulen in USA 189

Medizinstudent, Schweigepflicht und Fortbildung $781 \mathrm{R}$

Megaureteren 932

Melaena des Neu. geborenen 797

Melanom, Therapie 501

- Thormählensche Probe 919

Melanome und Heilprak tikerbehandlung $\mathbf{8 7 1} \mathrm{R}$

- und Herzmetastasierung 594

Melanosis Dubreuilh $\mathbf{5 0 1}$

Membrankrankheit, hyaline und Abnabelung 460

Menghini-Nadel zur Leberbiopsie 1160

Menière, Diagnose 409 
$\alpha$-Methyl-m-tyrosin- und Blutdruck 198

Methylprednisolon und Ulcustherapie 725

Michael, Stiftung 329

Mikrobiologie, medizinische $875^{*}$

Mikrographie nach Pallidotomie 988

Mikrosomen und Pharmaka-Entgiftung 292

Milch, Kriterien 919

Milz und Hepatitis chronica 1210

- und Werlhof 1069

Minderjährigkeit und Heileingriffe 324 R

Mineralien, Normgrenzen im Harn 325

Mineralstoffwechsel und Knochen 60

Mißbildungen, menschliche $1100^{*}$

Mißbräuche und Sucht $327^{\circ}$

Mitochondrien und Vererbung 717

Mitralstenosen, rezidive 433

Mongolismus und Röntgenstrahlen 329

-, Strahlenbelastung der Mütter 669

Mononucleose und Hanganutziu-Deicher 233

- und Lymphozytenkultur 413

- und Thrombozytopenie 400

Monosomie 1095

Morbus Addison 957

- - und Corticoide 725

- Bechterew und Laboratoriumsbefunde $\mathbf{2 1 9}$

- Bowen 909

- Cushing und Fettstoffwechsel 776

- - und Insulinresistenz 973

- Gaucher und Glucocerebrosidasemangel 236

- haemorrhagicus neonatorum $\mathbf{7 9 3}$

- Hodgkin 523

- - und Lymphographie 1178

- Menière, Diagnose 409

- Werlhof, Klinik und Immunologie 1069

Moronal bei Mykosen 714

Moroprobe und Isoniacidtherapie 1171

Mucormykosen $\mathbf{7 1 2}$

Mücken, „insect repellent" 728

Müttersterblichkeit, Rückgang 49

Multifidus-DreieckSyndrom 444

Multiple Sklerose, Invalidität 996

Murexid-Probe auf Barbitursäure 81

Muskeldurchblutungsstörung $\mathbf{8 6 4}$

Muskeldystrophie, progressive und Therapie 108

Muskeldystrophien $1136^{*}$

Muskelkrankheiten $45^{\circ}$

Muskelspannung und

Gammasystem 666
Muskeltraining, isometrisches $517^{\circ}$

Muskulatur, Pathologie $1136^{\circ}$

Mumps und Enzephalitis 282

Mutation und anabole Hormone 556

Mutationen und plasmatische Erbträger 717

Myasthenia gravis 396

Myasthenie, Prostigmin und Psychosyndrom 699

- und Thymus-Syndrom 1106

Mycoplasma pneumoniae, Bedeutung 1013

- - Erkrankungen 429

Mycostatika bei Mykosen 714

Mydriatica und Irispigmentation 285

Mydriatikum 666

Myelinisationsgliose 79

Myelitis und spinale Apoplexie $\mathbf{9 2 6}$

Myelographie und Querschnittsyndrom 1111

- bei spinaler Apoplexie 928

Myelomeningocele des Säuglings $\mathbf{8 0 1}$

Myelose, Differenzierung 1055

Mykosen und Erysipel $\mathbf{8 2 1}$

- der Lunge 711

-, Therapie $\mathbf{7 1 3}$

-, viszerale $787^{\circ}$

Mylepsinum bei Epilepsie 127

Myleran bei Polycythaemia 1004

Myokardinfarkt und Adenosindesaminase 166

-, Diät 705

-, Postmyokardinfarktsyndrom 1041

Myocardinsuffizienz Prüfung mit Kletterstufe $\mathbf{8 8 8}$

Myokarditis $1135^{\circ}$

- und Extrasystolie 872

- und Thymussyndrom 1108

Myokardkontraktion und Koronardurchblutung 1

Myokardose $1135^{\circ}$

Myopathie, thyreotoxische 536

Myopathien 45

Myxödem und Autoantikörper 441

Myxome 594

\section{$\mathbf{N}$}

Nabelblutung des Neugeborenen $\mathbf{7 9 9}$

Nachblutungen 1206

NADPH und Arzneimittelentgiftung 292

Nägeli-Preis 970

Naevus pigmentosus, Therapie 1098

Naevuszellnaevus, Therapie 1098

Nagelmykosen und Therapie $\mathbf{4 1 2}$
Nahrung und Hämorrhoiden 45

- und Ileus 305

Narkose und Blutgase 407

- und Estil 665 R

Narkoseantagonisten $46^{*}$

Nasaltest bei Asthma 903

Nase, Tumoren $46^{*}$

Nasenkrankheiten, Atlas $327^{*}$

Natamycin bei Mykosen 714

Natrium im Urin 325

Natulan und Polycythaemia vera $\mathbf{5 6}$

Naturforscher Leopoldina, Mitgliedswahl 329, 721, 1214

Nebenniere und Schilddrüse 691

Nebennierenlipom und Phäochromozytomsymptomatík 254

Nebennierenrindenadenome und $\mathrm{M}$. Cushing $\mathbf{1 7 4}$

Nebennierenrindenfunktion, Synacthen-Test 934

Nebennierenrindenhormone, Biochemie 515*

- und Fettgewebsstoffwechsel 776

Nebennierenrindenhyperplasie und Hypercorticismus 841

Nebennierenrindeninsuffizienz 957

Nembutal, Entgiftung 291

Neomycin bei Lebercoma 222

Nephritis, interstitielle chronische 1090

- und Nephrose 348, 363

- Pathogenese 551

Nephrocalcinose und Hypercalcämie $\mathbf{8 8 2}$

Nephron, Mikromorphologie $468^{\circ}$

Nephrose und kongenitale Glomerulonephritis 348, 363

- und ${ }^{131} \mathrm{~J}$-Trijodthyronintest $\mathbf{5 4 6}$

Nervenärzte und Notfalldienst $1169 \mathrm{R}$

Nervensystem, Anatomie 75

- und Koronardurchblutung 2

-, Pharmaka $46^{\circ}$

Nervosität und Hyperthyreose $\mathbf{1 1 4 5}$

Nervus obturatorius und Gelenkkontrakturen $\mathbf{3 1 3}$

Netzhautablösungen $920^{*}$

Netzhautchirurgie $921^{\circ}$

Neugeborene, Blutungsübel 793

-, Membransyndrom 423*

- und Nierenvenenthrombose 57

Neugeborenenerythroblastose, Amniozentese und Transfusion 229

Neugeborenes, Credésche Augenprophylaxe 467

- Herpes-simplexErkrankung 1183

Neuroanatomie und Medizin 75

Neurodermitis, Therapie 598

Neurologie, Lehrbuch 968

Neurotoxizität von

Streptomycin $\mathbf{1 1 5 6}$

Nicotin und Entwöhnung 233

Nicotinsäuretherapie und Stenocardie 725

Niederspannung im EKG 768

Niere und AmpicillinSpiegel 206

- und Aquocobalamin 1101

- und Coxsackie-Viren

1182

- und Cystinose 1124

- beim Diabetes 1137

-, doppelte und Bourneville-Pringlesche Phakomatose 491

-, - und Erkrankung 448

- und Fabry-Krankheit 243

- und kongenitale Glomerulonephritis 352,363

- und Hypertonie 329, 529, $563^{\circ}$

- und Ischämieverhalten des Nephron $468^{\circ}$

- und Säure-Basen-Regulation 406, 600

- und Schock, Pathologie 147

- und Streptomycinschäden am Ohr 1159

Nierenbecken und Harnleiterabgang 931

Nierenclearance $1211^{*}$

- und Aquocobalamin 1101

Nierenerkrankungen und Reninaktivität $\mathbf{1 0 7 5}$

Nierenfunktion, Physiologie und Pathophysiologie $1173^{\circ}$

- und Radioisotope $469^{\circ}$

Niereninsuffizienz und Achillessehnen-Reflexzeit $\mathbf{7 6 3}$

- bei Diurese 726

Nierenleeraufnahme bei 
Noradrenalin im Zentralnervensystem $\mathbf{3 1 8}$

Normalwert und Laboratorium 1061

Notfalldienst für Nervenärzte $1169 \mathrm{R}$

Notfallfibel, kinderärztliche $422^{\circ}$

Notfallvertretungsdienst $416 \mathrm{R}, 417 \mathrm{R}$

- von Fachärzten 366 R

- durch Nichtkassenärzte 826. $R$

Nucleotide bei Muskeldystrophie 115

Nystagmus und Menière 409

Nystatin bei Mykosen $\mathbf{4 1 2}$, 714

O

Obstruktionsileus nach Magenresektion 303

- nach Operationen 624

Osophagus, Krankheiten des chirurgischen Fortschrittes 624

Osophagusstenose beim Kind 1209

Ösophagusvarizen,

blutende 991

- - - und Tamponade 422

-,- und Zirrhose 686, 1207

- und Glucocorticoidtherapie 466

17-OHCS und Anorexia nervosa $\mathbf{5 0 0}$

Ohr und Streptomycinschädlen 1159

Ohrenkrankheiten, Atlas $327^{\circ}$

Operation und Blutungen 1205

- und Einwilligung $323 \mathrm{R}$

-, fehlerhafte und Verantwortlichkeit $365 \mathrm{R}$

-, Fremdkörperzurücklassung $463 \mathrm{R}$

- und Lungenfunktionsprüfung 658

- von Mitralstenosen 435

- und Schockniere 149

Operationen, gynäkologische $188^{*}$

- Zwischenfälle 283

Operationslehre, allgemeine und spezielle $188^{\circ}$

- unfallchirurgische $564^{*}$

Operationstrakt, Sicherheit $47^{*}$

Operationswunden, Nachblutung 466

Operator und Proteinsynthese $\mathbf{1 8 1}$

Ophthalmologie und Antikonzipienten $469^{*}$

- Probleme 920*

Ophthalmotest bei Asthma 903

Optiker und Sehschärfenbestimmung $720 \mathrm{R}$

Organtransplantation Stand 377

Ormondsche Krankheit 97

Orthopädie, fortgeschrittene $787^{\circ}$
Orthopädie, kleine $139^{\circ}$

-, Lehrbuch $187^{*}, 236^{*}$,

$284^{\circ}$

Ortolani-Zeichen bei

Hüftgelenksluxation $\mathbf{1 6 9}$

Ospolot bei Epilepsie 127

Ossovenographie 929

Osteoporose und Calcium-

bestimmung 60

- nach Magenresektion 846

-, Physiopathologie und

Therapie 1079

Oszillographie und Gefäßprozesse 784

Ovar, Endokrinologie $831^{*}$

Ovarialfunktion und

Verfolgung $\mathbf{2 6 0}$

Ovulationshemmer, Bezeichnung 1098

- und Blutkoagulabilität 187

-, hormonale und Vitamin $\mathrm{B}_{6} 1174$

- und Mehrlingsgeburt 1134

- und Progesteronüberempfindlichkeit 399

Ovulationshemmung durch Hormone $830^{\circ}$

Oxacillin bei Nierenversagen 1035

Oxalaturie und Stein-

bildung 326

Oxalsäure im Urin 325

\section{$\mathbf{P}$}

${ }^{32} \mathrm{P}$ und Polycythaemia vera 55

Pädologie, Fortschritte $786^{*}$

Pagitane bei Parkinson 550

PAH-Clearance und Ampicillin 208

Pallidotomie bei Epilepsie 986

Panarteriitis nodosa, Serologie 220

Pankreas und CoxsackieVirusinfektion 1183

- und Diabetes juvenilis 779

-, Krankheiten nach Operationen $\mathbf{6 2 5}$

- und labiler Diabetes 690

- und Papillenstenose 661

Pankreasadenom, seltenes 634

Pankreasdiagnostik 377

- mit Radioisotopen 1122

Pankreasfisteln, Therapie 643

Pankreasnekrose und Fistelbildung 644

Pankreaspseudozysten und Pleuraergüsse 783

Pankreasschädigung nach Magenresektion $\mathbf{8 4 4}$

Pankreasszintigramm 1123

Pankreaszirrhose und Diabetes 377

Pankreatektomie 624

Pankreatitis $139^{\circ}$

-, chronische 1208

Pantothensäure und Streptomycinentgiftung 1151

Papierchromatographie auf Barbitursäure 81
Papierelektrophorese und Leberdiagnostik $\mathbf{2 6 7}$

Papilla Vateri, Stenosen 660

Papillenstenosen, Therapie 660

Parabene bei Mykosen $\mathbf{7 1 4}$

Paracelsus-Medaille 1101, 1174

Paraganglien, Tumoren $\mathbf{4 9 3}$

Parahämophilie $\mathbf{2 7 0}$

Paraproteinämien und Enzephalopathie $\mathbf{6 7 5}$

Parasympathikus und Herz

Parathion, Entgiftung 295

Paratyphus, Problematik 803

Parkinsonsche Krankheit und biogene Amine 318

- - und Pallidotomie 988

- - Therapie $\mathbf{5 5 0}$

Parodontose, Therapie 967

Paronychia candidamycetica und Pimaricin 253

Parpanit bei Parkinson $\mathbf{5 5 0}$

Passage, maternofetale und fetmaternale $\mathbf{1 2 9}$

Pathologie, Datenverarbeitung 1174

-, experimentelle $328^{\circ}$

- Heidelberg, Lehrstühle 92

- von Nierenerkrankungen und Schockniere 149

PBJ-Bestimmung $\mathbf{5 4 1}$

Pemphigoid 1073

Pemphigus, Therapie $\mathbf{1 0 9 3}$

- vegetans 1093

Penicillin, Allergie 999

- und Dermatomykosen 412

- bei Erysipel 821

- bei Erythema chronicum migrans 233

- und Streptomycin 1150

Penicilline und Mykosen 714

Penicilloyl-Polylysin, Hauttest $\mathbf{1 0 0 0}$

Pensionskassèn $48^{\circ}$

Pentobarbital, Entgiftung

Peptidsynthese, automatische 1137

Perikarditis und Postmyokardinfarktsyndrom $\mathbf{1 0 4}$

- durch Tumormetastasen 594

Perinatalperiode und Pneumonieformen $\mathbf{2 7 3}$

Peritonealdialyse und Hämodialyse $\mathbf{7 0 6}$

Peritonitis nach Ileus 954

- und Schockniere 150

Perkussion und Osteoporose 63

Permeabilitätsfaktoren $\mathbf{3 3}$

Perniziosa und Blutgruppe 48

Pertussis und Masernimpfstoff $\mathbf{5 7 2}, \mathbf{5 7 5}$

Pestjahr London 1665 1063*

Pflegepersonal in Krankenhäusern 669

-, Zahl 285

Pflegschaft bei Psychosen $324 \mathrm{R}$

Pfortaderhochdruck 1207

ph-Bestimmung des Harns

Phäochromozytom und Insulinresistenz $\mathbf{9 7 3}$

Phäochromozytomsymptomatik und Nebennierenlipom 254

Phagozytenfunktion der Hassalschen Körperchen 189

Phakomatose, BournevillePringlesche $\mathbf{4 8 8}$

Pharmaka, Entgiftung $\mathbf{2 8 9}$

- und Leber $188^{\circ}$

Pharmakologie 1211

-, Aussichten 140

-, experimentelle $46^{*}, 96^{*}$

- und Koronarzirkulation 727

- Lehrbuch 611.

Pharmakotherapie, psychiatrische $467^{*}$

Phenobarbital, Entgiftung

291

- und Epilepsie 127

Phenothiazine, Linsentrübuing 517

Phenoxybenzamin und Schock 43

Phenylalanin, Bestimmung $235^{\circ}$

Phenyramidol $469^{*}$

Phosphat im Urin 326

Phosphatasen, alkalische und Blutgruppen $\mathbf{5 0 7}$

- und Pleurodynie $\mathbf{1 1 8 2}$

-, Bestimmung 235*

- Stabilität $\mathbf{8 5 2}$

Phosphatausscheidung im Urin 601

Phosphatfructokinase und Blutungsübel $\mathbf{7 9 5}$

Phosphatide und Arteriosklerose $\mathbf{8 6 2}$

Phosphatidspeicherkrankheit 241

Phosphoglucomutase in Fett und Leber $\mathbf{7 7 4}$

6-Phosphogluconat-Dehydrogenase-Aktivität bei Collumkarzinom $\mathbf{3 4 5}$

_ _ - in Fett und Leber 774

- - - in Thrombozyten 795

Phosphoglyceratkinase und Thrombozyten $\mathbf{7 9 5}$

Phosphoglyceromutase in 
Plasma und Gerinnungsdefekte $\mathbf{3 1 6}$

-, Reninaktivität 1075

-, Zytogenetik 715

Plasmafraktion I bei Neugeborenenblutung $\mathbf{8 0 0}$

Plasmakinine und Entzündung 35

Plasmon, Funktion und Struktur $\mathbf{7 1 8}$

Plasmozytom und Enzephalopathie 677

- und Lymphozytenkultur 413

Plastiden, Rolle $\mathbf{7 1 6}$

Plazenta und Transfusion

Plazentalokalisation mit Infrarotthermographie 962

Pleura und Gewebspunktion 376

Pleuraerguß und Pankreaspseudozysten 783

Pleurodynie 1180

Pleuropneumonie durch Mycoplasma 1013

Plexus chorioideus und Liquorsekretion $\mathbf{8 6 7}$

Pneumokokken, Therapie 85

Pneumencephalogramm $188^{\circ}$

Pneumometrie-Test beim Asthma 904

Pneumonektomie, Folgekrankheiten $\mathbf{6 2 5}$

- und Lungenfunktionsdiagnostik 659

Pneumonie nach Ileus 954

-, interstitielle 275

- konnatale 273

- mit Mycoplasma pneumoniae 429, 1015

- in der Perinatalperiode 273

- plasmazelluläre und RS Viren 158

Pneumotachographen, Wert 514

Pneumothorax, spontaner und Bourneville-Pringlesche Phakomatose $\mathbf{4 8 8}$

Pocken, Therapie $\mathbf{4 5 5}$

Poliomyelitis und Masernimpfstoff 572, 575

- Statistik 1009

Poliomyelitisimpfung, Virämie 385

Pollenasthma, Desensibilisierung 906

Polyarthritis chronica und Laboratoriumsbefunde $\mathbf{2 1 9}$

- und Rheumafaktor 514

Polycythaemia vera und Enzephalopathie 677

- - Therapie 1004

- - und Zytostatika 55

Polyneuritis und Polyneuropathie 1193

Polyneuropathie, Therapie 1194

-, Diagnose 1192

Polypeptide, basische und Entzündung 35

Ponalid bei Parkinson 550

Poplitea-Verschluß 813

Porphyrie, intermittierende und Therapie 959
Postcholezystektomiesyndrom 624

PostmyokardinfarktSyndrom 1040

Potenzminderung, Therapie 234

Prädiabetes $\mathbf{7 4 5}$

- der Mutter und Fetus 217

Präparateprüfung, Steuerbegünstigung $609 \mathrm{R}$

Prednison bei Hyperthyreose $\mathbf{4 4 2}$

- und Nierentransplantation $\mathbf{7 4 1}$

Prick-Test beim Asthma 903

Pringlesche Phakomatose 488

Procain und Lungenödem 1202

Progesteron, Uberempfindlichkeit bei Prurigo 398

Proluton, Uberempfindlichkeit 399

Prosektoren, nichtärztliche in USA 381

Prostaglandine, Regulation 1083

Prostatakarzinom, hämorrhagische Diathese 119

Prostataoperationen und Fibrinolyse 297

Prostigminbehandlung bei Myasthenie 699

Protein, c-reaktives und Aussagewert 514

-, unbekanntes 425

Proteinbildung in Lymphozytenkultur $\mathbf{3 6 2}$

Proteine, Biosynthese $\mathbf{1 7 9}$

-, Evolution menschlicher 831"

Proteinurie bei FabryKrankheit $\mathbf{2 4 1}$

Proteus und Streptomycin 1153

Prothrombin und Neugeborene $\mathbf{7 9 4}$

Prothrombingruppen und Gerinnungsdefekte $\mathbf{3 1 6}$

Provokationsproben beim Asthma 903

Pruritus ani bei Hämorrhoiden 45

Prurigo und ProgesteronÜberempfindlichkeit 399

Pseudomonas testosteroni und Steroide 184

Psyche und Krankheiten 968

Psychiatrie, Arzte und Notfalldienst $1169 \mathrm{R}$

- hirnorganischer Störungen $379^{*}$

-, Krankenhaussicherungen $416 \mathrm{R}$

- und Pharmakotherapie $467^{\circ}$

Psychoanalyse und Depression 421

Psychopathologie und EEG 228

-, klinische 785

Psychopharmaka $467^{\circ}$

Psychosen und Heil eingriff $323 \mathrm{R}$

- und Krankheiten $968^{*}$

-, Phenothiazine und Linsentrübung 517

Psychosyndrom und Myasthenie 699
PTA-Faktor, Fehlen 269

Publikationen, medizinische 1008

Pulmonalstenosen und Hypercalcämie $\mathbf{8 8 2}$

Puls und Kletterstufe $\mathbf{8 8 7}$

- und Rezeptorenblocker 146

Pupillenerweiterung, medikamentöse 666

Purinethol bei Leukosen 246

Puromycin und Gedächtnis 1060

Purpura und Enzephalopathie 676

-, idiopathische 1069

Pyelogramm bei Doppelniere $\mathbf{4 5 1}$

Pyelonephritis 1090, $1173^{\circ}$

- und Acidose 603

-, Fortschritte 1006*

- und Schockniere 149

Pyloroplastik 648

Pylorusstenose beim Kind und Kardia 308

und Plastik 654

Pyocyaneus und Streptomycin 1153

Pyridostigmin bei Myasthenie 699

Pyruvatkinase in Leber und Fett $\mathbf{7 7 4}$

- in Thrombozyten 795

Pyurie im Kindesalter. Diagnostik 930

\section{$\mathbf{Q}$}

Q-Zacke, Beurteilung 859

- im EKG 15

- und Infarkt 980

Quarantäne, erste 1134

Quark, Methionin- und

Cholingehalt 1134

Queckenstedtversuch doppelter $\mathbf{1 1 1 0}$

- und Liquorstop 928

Quecksilber bei Grippe $\mathbf{3 2 5}$

Qecksilberdiuretika und

Säure-Basen-Haushalt 605

Quecksilberschmierkur und multiple Sklerose 1133

- bei Rötelnembryopathie 919

Querschnittslähmung bei spinaler Apoplexie 925

Querschnittssyndrom und

Therapie $\mathbf{1 1 0 9}$

QRS-Komplex $\mathbf{7 6 8}$

$\mathbf{R}$

Rachitisprophylaxe, Herzund Gefäßerkrankungen 883

Radar und Mongolismus 329

Radio-Bengalrosa-Test und Bromsulphaleintest 211

${ }^{198}$ Radiogold zum Leberszintigramm 820

Radioisotope für Hirntumornachweis $\mathbf{4 5 4}$

- zur Leberdiagnostik 819

- und Osteoporose 63

- zur Pankreasdiagnostik 1122

Radioisotopen-Renographie 469*

Radiojodfunktionstest und Schilddrüse 103

Radiojodstoffwechsel und Hyperthyreose $\mathbf{1 1 4 8}$

Radiojodtherapie und Autoantikörper $\mathbf{4 4 0}$

Radiologie, Handbuch 468*

Radiophosphor, Makroradiographie bei Magenkarzinom 90

- und Polycythaemia vera 55

Ratte und Leptospirosen 370

Rauchen und Blasenkrebs 565

- und Bronchuskarzinom 788

- und Emphysem 85

- und Gesichtsfeld 481

Reaktionen, enzymatische 786*

Rechtschreibe-Schwäche 461

Rechtshändigkeit in der Schule 783

Recurrenslähmung und Strumaoperation 1207

Redeker-Preis 1965329

Reflexzeit ud Serumkalium 764

Reflux, vesiko-uretraler beim Kind 931

Refluxösophagitis beim Kind 1209

Regelkreis und Koronardurchblutung 6

Regulationen in der Medizin 1164

Regulations-Tests 1167

Rehabilitation und Rentenversicherung 49

Renin und Hypertonie 329

Reninaktivität im Plasma und Blutdruck 1074

Renographie und Radioisotope $469^{*}$

Rentenversicherung und Facharztausbildung 230 R

- und Rehabilitation 49

Reparit bei Hirndruck- 
Retinopathia diabetica, Therapie 306

Retinopathie und Diabetes 749

Rezeptorenblocker bei Lungenkrankheiten 145 Rezeptur 423*

Rezidivmitralstenosen $\mathbf{4 3 3}$

Rezidivstruma 1205

Rezirkulationsperitonealdialyse $\mathbf{7 0 6}$

Rheinland, Assistentenordnung $781 \mathrm{R}$

Rheographie und Gefäßprozesse 784

Rhesusfaktor und Geburtenfolge 969

-, Suche 666

Rhesus-Inkompatibilität, Entwicklung 610

Rhesus-Untergruppe und Kreuzprobe 186

Rheumaerkrankungen und Laboratoriumsbefunde 219

Rheumafaktor, Aussagewert 514

Rheumafaktoren 220

Rheumatismus, Verhütung und Therapie $328^{\circ}$

Ribonucleinsäure und Steroide $\mathbf{1 8 4}$

Ribonucleinsäure-Stoffwechsel 728

Ribosomen und Proteinsynthese 183

- und Seitenkettentheorie 1197

Rickettsieninfektionen des Menschen $423^{*}$

Rinder-Insulin und Insulinresistenz $\mathbf{3 1 5}$

Röntgenanalyse von Lungenerkrankungen $235^{\circ}$

Röntgenbeurteilung der Osteoporose 61

Röntgendiagnostik $667^{*}$

- Lehrbuch $874^{\circ}$

Röntgenologe und EKG $510 \mathrm{R}$

-, Fachgebietsbeschränkung $463 \mathrm{R}$

Röntgenologe und Umsatzsteuerpflicht $1169 \mathrm{R}$

Röntgenphysik $234^{*}$

Röntgenpraxis, Herzvolumenbestimmung $327^{*}$

Röntgenstrahlen und Mongolismus 329

Röntgenuntersuchungen bei Multifidus-DreieckSyndrom 446

Röteln und Embryo 1008

RS-Virus beim Kind 53, 153

Rubeolenembryopathie, Quecksilberschmierkur 919

Rudimentärinfarkte $\mathbf{9 8 3}$

Rückenmark, Anatomie 75

-, Angiome 173

- Apoplexie 925

Rückenmarksverletzungen, Differentialtherapie $\mathbf{1 1 0 9}$

Rückenschmerzen und Multifidus-DreieckSyndrom 445

Ruhr durch Amöben 138

"runt disease" 131

Ryanvirus, Pathogenität
S

Sabin-Feldman-Test bei Toxoplasmose 391

Sabin-Impfstoff und Virämie 385

Sachverständiger, gerichtlicher $\mathbf{7 8 0} \mathrm{R}$

Säugling und Akkomodation 1064

- und Kardiafunktion 311

- und kongenitale Glomerulonephritis $\mathbf{3 6 3}$

-, moropositiver und INHBehandlung 1171

- und Nierenvenenthrombose 57

Säuglingssterblichkeit, Rückgang 49

Säure-Basen-Haushalt, Harnpufferanalyse 403

Säure-Basen-Regulation, renale 600

Säureverätzung der Augen 469

Salmonella infantis Meningitis 892

Salmonellaerkrankungen, Probleme $\mathbf{8 0 3}$

Salmonellen in der Großstadt $48^{*}$

Salmonellenmeningitis beim Erwachsenen 891

Salpingolysis 1047

Salpingitiden nach Verfolgung 260

Sanamycin und Nierentransplantation $\mathbf{7 4 1}$

Sanarelli-ShwartzmanPhänomen und Kälteschäden 285

Sarkoidose und Lymphozytenkultur $\mathbf{4 1 3}$

Sauerstoff und Koronardurchblutung 3

- und Lungenfunktionsprobe 660

Saugbiopsie des Magens 1134

Saugbiopsiematerial Erythrozytenentfernung 95

Schadenersatz bei Fachgebietüberschreitung $91 \mathrm{R}$

Schädelhirntrauma $\mathbf{1 1 2 6}$

Schädelprellung und Schleuderung $\mathbf{5 8 8}$

Schädelunfall und EEG 226

Schädelverletzung und epidurale Blutung 1186

Scharlach, Neuerkrankungen 1009

Schaumann, O., 75. Geburtstag 724

Schilddrüse und Diabetes 691

- und Fettstoffwechsel 777

Schilddrüse und Hyperthyreose $\mathbf{1 1 4 4}$

- und Myopathie 536

- und Thyreocalcitonin 833

Schilddrüsenadenome, Chirurgie 1206

Schilddrüsendiagnostik $\mathbf{5 4 1}$

Schilddrüsenerkrankungen, Achillessehnenreflex 101

- und Autoantikörper 437

Schilddrüsenfunktion und Niereninsuffizienz $\mathbf{7 6 7}$
Schimmelpilze und Pimaricin 252

Schlafmittel, Entgiftung 291

Schlafmittelvergiftung und Analeptika 514

- und Magenspülung 485

Schleudertrauma der Halswirbelsäule 388, 1209

Schluckimpfung und Virämie $\mathbf{3 8 5}$

Schmerzensgeld bei Fremdkörperzurücklassung $463 \mathrm{R}$

Schmerzensgeldbeiträge $787^{\circ}$

Schock, anaphylaktischer 95

-, Differenzierungen 1043

Schockniere und Pathologie 147

Schock therapie und Vasodilatation 43

Schöpfungstag und Mensch der Zukunft $1173^{\circ}$

Schrift und Pallidotomie 987

Schrumpfniere 1091

Schule und Linkshänder 783

Schvabe-Preis 1965329

Schwachsinn bei Bourneville-Pringlescher

Phakomatose 491

Schwangerschaft, Blutübertritte 130

- und Diabetes des Kindes 216

- und Ikterus 1114

- und Lymphogranulomatose $\mathbf{5 2 2}$

- und Virusimpfung 1008

Schwangerschaftsunterbrechung, Recht $277^{\circ}$

Schweden, Physiologie 911

Schweigepflicht und Arztbefund - Weitergabe $1056 \mathrm{R}$

- des Medizinstudenten $781 \mathrm{R}$

Schweineinsulin und Insulinresistenz 315, 975

Schweißerkrankheit 873

Schwerhörigkeit bei

Dystrophia myotonica 947

- und Streptomycin 1155

$\mathrm{SDH}$ und Pleurodynie 1182

- Stabilität 853

Seborrhoe, Therapie $\mathbf{5 9 8}$

Sehschärfe, Bestimmung durch Optiker $720 \mathrm{R}$

Seitenkettentheorie Ehrlichsche und Wandlung 1197

Sekretin und Magenresektion 845

Sekretionsanalysen der Magenschleimhaut 356

Sekretolyse bei Emphysem 85

Sekundenkapazität 659

Selektionshypothese von Jerne und Burnet 1198

${ }^{75}$ Selen-L-Methionin zur Pankreasdiagnostik 1122

Sendai-Pneumonie 276

Septum des Herzens und Q-Zacke 20

Serologie der Haut- und Geschlechtskrankheiten $96^{\circ}$

Serotonin und Entzündungsstoffe $\mathbf{3 4}$

Serum, Adenosindesaminase-Bestimmung 159

- und Insulinbindung 856

Serumaktivität von Fermenten 831

Serumcholesterin und Arteriosklerose $\mathbf{8 6 2}$

- und Hyperthyreose 1148

Serumdiastase 784

Serumeisen und Polyarthritis 219

Serumfettsäuren und Stress 194

Serumharnsäure, Rolle 921

Serumhepatitis und

Zirrhose 685

Serumhormonjod und Kropf 542

Serumkalium und Achillessehnenreflex $\mathbf{7 6 4}$

Serumkupfer und Polyarthritis 219

Serumlabilitätsproben und Leber 267

Serumlipoide bei Arteriosklerose 861

Seuchengesetz $380^{*}$

Sexualerziehung der Jugend $96^{\circ}$

Sexualhormone und Fettstoffwechsel 777

- und Koronarinsuffizienz 44

Shunt, splenorenaler bei Zirrhose 686

Shuntoperation bei Pfortaderhochdruck 1207

Sichelzellanämie und Zentralnervensystem $\mathbf{6 7 4}$

Sicherheit im Operationstrakt $47^{\circ}$

Silubin retard bei Diabetes 939

single ventricle und Q-Zacke 20 
Sozialrecht in der Medizin 876*

Sozialrichter für Kassenarztsachen $558 \mathrm{R}$

Sozialversicherungsträger, Prüfung der Selbstkostenrechnung $509 \mathrm{R}$

Spätgestose, Ikterus 1114

Speisen und Ileus $\mathbf{3 0 5}$

Spermiogenese und Spurenmetalle 1064

Sphinkter im Kardiabereich beim Kind $\mathbf{3 1 1}$

Sphinkterotomie der Papilla Vateri 661

SP-I und Choriokarzinom

Spinalerkrankungen, apoplektiforme $\mathbf{9 2 8}$

Spirographen 725

Spirometrie und Operation 658

Spironolacton und SäureBasen-Haushalt 605

Splenektomie bei hämolytischem Ikterus $\mathbf{4 7 5}$

- und Nierentransplantation $\mathbf{7 4 0}$

- bei M. Werlhof $\mathbf{1 0 7 3}$

Splenomegalie, Laparoskopie 1162

Spontanpneumothorax und Bourneville-Pringlesche Phakomatose $\mathbf{4 8 8}$

Sporotrichose $\mathbf{7 1 2}$

Sport und Atmung 1212*

- und Kyphose 44

Spülbehandlung bei Nephrolithiasis $\mathbf{1 0 2 4}$

Spurenelemente und Anacidität 282

Spurenmetalle und Spermiogenese 1064

ST, Hebung und Infarkttherapie $\mathbf{9 8 0}$

Standardmethoden in der klinischen Chemie $235^{\circ}$

Stapenor bei Nierenversagen 1035

Staphylokokken in der Klinik 425

- und Streptomycin 1153

Staphylokokkensepsis und Nierenversagen 1035

Statistik und Karzinogenese $135 \mathrm{R}$

- klinische 1211

- und Krebs $328^{\circ}$

-, medizinische 1088

Status epilepticus, Therapie $\mathbf{1 2 8}$

Staub-Traugott und latenter Diabetes $\mathbf{7 6 0}$

Stenocardie und Nicotinsäuretherapie 725

Stenose der Aorta, Therapie 10

Stereotaxie-Eingriffe bei Epilepsie 984

Sterilität, weibliche $\mathbf{1 0 4 7}$

Steroide und Enzyminduktion 182

- und Leberzirrhose 685

- bei Leukosen 246

Steroidhormone, Wirkungsmechanismus $\mathbf{1 7 8}$

Steroid-Myopathie 111

Stethoskop, Verstärkerauskultation 829

Steuer nach Verkehrsunfällen 139
Steuerbegünstigung bei Präparate-Prüfung $609 \mathrm{R}$

Steuervergünstigung und Wissenschaftsarbeit $721 \mathrm{R}$

Stickstoff im Urin 325

Stickstoffdioxid und Lunge 899

Stickstofflost und Insulinresistenz 976

- und Lymphozytenkultur 414

Stiegele-Forschungspreis 1064

Stilbamidine bei Mykosen 714

Still-Felty-Syndrom Serologie $\mathbf{2 2 0}$

Stimmstörungen und anabole Hormone $\mathbf{5 5 5}$

Stoffwechsel und Enzyme $1100^{*}$

- im Fettgewebe $\mathbf{7 7 3}$

- des Kindes $1172^{\circ}$

-, pathologischer $564^{*}$

$\rightarrow$, Regulationen 832*

Stoffwechselstörung, diabetische $\mathbf{7 4 6}$

Stouffer-Preis 1064

Straftaten und Meldepflicht des Arztes $42 \mathrm{R}$

Strahlen 234*

Strahlenbelastung und Mongolismus 669

Strahlenschäden und Sorgepflicht $1058 \mathrm{R}$

Strangulation im Alter 953

Straßenverkehrsunfälle 1965613

Streptokinase bei Herzinfarkt 581

Streptokinasetherapie des Herzinfarkts $\mathbf{9 7 8}$

Streptokokken und Glomerulonephritis $\mathbf{5 5 2}$

Streptomyces und Steroide 182

Streplomycin, Entgiftung 1150

Stress und Fettsäuren 193

Struma und Achillessehnenreflex $\mathbf{1 0 1}$

- Hashimoto 437

- und Hyperthyreose 1146

-, Rezidive 1205

Stuart-Power-Faktor $\mathbf{2 7 0}$

- - - Mangel 793

Stufenbelastung und Koronarreserve $\mathbf{8 8 4}$

Stuhlfettbestimmung 829

Subaortenstenose, idiopathische und Q-Zacke 859

Subarachnoidalblutung und spinale Apoplexie 927

- und EEG 226

Sucht und Mißbrauch $327^{*}$

Sulfonamide und Dermatomykosen $\mathbf{4 1 2}$

- bei Mykosen 714

Sulfone und Lepra 607

Sulfonylharnstoffe und Fructose bei Diabetes 306

- und Insulinresistenz 975

Suxinutin bei Epilepsie 127

Sympathektomie und Durchblutungsstörung 813, 864
Sympathikus und Herz 4

Sympathikuschirurgie der Gefäßverschlüsse 1207

Synacthen-Kurztest 934

Syndrom, bronchitisches $140^{*}$

Synchronisation vegetativer Funktionen 610

Syndrom, adrenogenitales und paraneoplastischer Hypercorticismus $\mathbf{8 4 1}$

-, apallisches 1126

- hepatorenales und Acidose 604

Syphilis, Therapie $96^{\circ}$

Szintigramm der Leber $\mathbf{8 1 9}$

- bei Pankreasdiagnostik 1123

Szintigraphie der Lunge 333

$\mathbf{T}$

Tabak, Konsum 141

Tabakrauchen, Entwöhnung 233

Tacholiquin und Sekretolyse 85

Tätigkeit, freiberufliche der Chefärzte $\mathbf{7 8 0} \mathrm{R}$

Taubheit und Streptomycin 1155

Tauchen und Schädlichkeit 187

$99 \mathrm{~m}$ Technetium für Hörtumordiagnostik $\mathbf{4 5 5}$

Technik der Histologie 1135

Teertherapie des Ekzems 599

Tegretal bei Epilepsie 127

Telecaesiumtherapie des Kehlkopfkrebses $\mathbf{3 3 9}$

Telefon, abgestelltes beim Arzt 826 R

Telekobalttherapie des Kehlkopfkarzinoms $\mathbf{3 4 0}$

Teleologie und Naturforschung 295

Temperatur, Regelkreisschema 1165

Temporalepilepsie, Therapie $\mathbf{1 2 7}$

Tendomykose $\mathbf{4 4 4}$

Teratogenese $1100^{\circ}$

Testosteron und Bakterienzelle $\mathbf{1 8 3}$

- und Kehlkopf $\mathbf{5 5 6}$

Testoviron und Potenz minderung 234

Tetanie nach Citraten 1004

Tetanus, Grundimmunisierung 966

- und unterlassene Impfung $558 \mathrm{R}$

- und Masernimpfstoff 575

Tetanustoxoid, Reaktionsabschwächung 788

Tetrachlorkohlenstoff und Antikoagulantien 669

Tetracyclin und Lebercoma 222

- und OsteoporoseDiagnostik 62

Tetraploidien 1094

Thalamotomie bei Epilepsie 985

Thalidomid, Entgiftung 293

- Immunsupression 1132

THAM, Bestimmung im Urin 405

Theobromin in der Kaffeebohne 49

Therapie, antiepileptische 126

-, experimentelle 613

- Fibel 96"

- der Kinderkrankheiten $235^{*}$

Thermoregulation in der Medizin 1164

Thiamin und Alkoholabbau 236

Thiazide und Dünndarmgeschwüre 131

Thiopental, Entgiftung 292

Thiotepa und Choriokarzinom 68

Thormählensche Probe 919

Thrombolyse beim Herzinfarkt, EKG 978

Thrombolysetherapie des Herzinfarkts $\mathbf{5 8 1}$

Thrombopenie bei M. Werlhof 1069

Thrombosen der Hirnvenen 1033, 1049

- und Kompressionsverband $\mathbf{7 4}$

Thrombozyten und Enzyme 795

Thrombozytenlebensdauer bei $M$. Werlhof $\mathbf{1 0 7 0}$

Thrombozytenübertragung 1073

Thrombozytopenie bei Mononucleose $\mathbf{4 0 0}$

- des Neugeborenen 793

Thymektomie $\mathbf{8 8}$

Thymoltrübungstest und Leber 268

Thymom 1108

und Herzmetastasierung 594

Thymus und Autoimmunkrankheiten 477

- und Bursa fabricii 88

- und Immunbiologie $47^{*}$

- und Immunität 87 
Toxocara canis bei Menschen 848

Toxoplasmose $1006^{*}$

- bei Kleinkind 309

Tragzeit und unterschrittene Empfängniszeit 117

Training, autogenes $379^{\circ}$

Transaminaseaktivität, Haltbarkeit $\mathbf{8 5 2}$

Transfusion, intrauterine bei Erythroblastose 229

-, plazentare 450

- und Rhesus-Untergruppen 186

Transhydrogenase-Therapie der Steroidhormone $\mathbf{1 7 9}$

Transplantation und Chirurgie 623

- und Lymphozytenkultur 415

- der Niere 738, 1007.

- und Thalidomid 1132

Transplantationsproblem in der Chirurgie 619

Trasylol und Gerinnung 317

- nach Prostataoperationen 297

Trauma und Achillessehnenriß 611

- und Diabetes 695

Traumatologie, Lehrbuch $187^{*}, 284^{*}$

- Praxis $667^{\circ}$

Tremaril bei Parkinson $\mathbf{5 5 0}$

Trenimon und Choriokarzinom 68

Trepanation und Nachbehandlung 1191

Triamteren und SäureBasen-Haushalt 605

Trichomonadeninfektion, orale? 282

Trichophyton und Pimaricin 251

Trichophytie, Therapie $\mathbf{4 1 2}$

Tridione bei Epilepsie 127

Trigeminusneuralgie 321

Triglycerid, Synthese 1084

- - - und Glucosestoff wechsel 773

Triploide und Abort 1094

Trisomien und Abort 1095

Trispuffer bei Säureverätzung der Augen 969

Tryptophanbelastung unter Ovulationshemmern 1174

Tubenverschluß, Wiederherstellung 1047

Tuberkelbakterien, Experimente $46^{\circ}$

Tuberkulinprobe und INH Therapie beim Säugling 1171

Tuberkulose und BCGImpfung 479

- der Doppelniere 449

-, Häufigkeit 1958 283.

- und Lymphogranulomatose $\mathbf{5 2 8}$

-, Rückgang 49

- und Streptomycin 1151

Tumor und Epilepsie, Elektroenzephalogramm.

Tumoren des Gesichtsschädels $46^{*}$

- und Herzgeschwülste 593

- des Magens und Fiberskop 1045
Tumoren der Paraganglien 493

-, vertebrale 925

Tumordiagnostik mit Infrarotthermographie $\mathbf{9 6 2}$

Tumorileus im Alter 953

Tumorübertragung durch Nierentransplantation 381

Tumorverdoppelungszeit bei Bronchuskarzinom 335

Tumorzellen und Erythrozytenabtrennung $\mathbf{9 5}$

Tympanicumkörperchen und Tumorbildung 494

Typhus, Problematik 803

-, Statistik 1009

\section{U}

UDPG-Pyrophosphorylase in Fett und Leber 774

Uberspannung im EKG 769

Ulcus und Blutgruppe $\mathbf{5 0 8}$

- duodeni und Vagotomie 648

- molle 96.

- und Stress 1206

- ventriculi dissecans $\mathbf{1 1 2 1}$

Ulcusentstehung und Methylprednisolon 725

Ultraschall und Hypophysenadenome 177

Umsatzsteuerpflicht des Krankenhausröntgenologen $1169 \mathrm{R}$

Unfall und Arzt $564^{\circ}$

- und Kahnbeinpseudarthrose 421

- und Schockniere 150

- und Unfallkrankenhaus $664 \mathrm{R}$

Unfallchirurgie $564^{*}$

- Lehrbuch 468*

Unglücksfälle und Hilfeleistungspflicht 1212

Universität Uppsala, Physiologie 916

Uppsala, Universitätsklinik 912

Urämie und Schockniere 150

Urbason bei Leukosen 247

Ureter bifidus $\mathbf{4 5 0}$

Ureterocelen $\mathbf{9 3 2}$

Urethritis $96^{\circ}$

Urin, Phosphatausscheidung 601

Urinausscheidung von Mineralien 325

Urogramm 931

Urokinaseaktivität nach Prostataresektion $\mathbf{3 0 0}$

Urolithiasis bei Doppelniere 449

Urologie, Handbuch $564^{\circ}$

-, Nachblutungen 1206

USA, Medizinschulen 189

Uterus und Sterilität 1047 $1006^{*}$

\section{V}

Vagina und Sterilitätsursachen 1048

Vagotonie und Nachkrankheiten 624

- und Ulkus 648
Vakzine-Antigen bei Pocken 457

Varizen, Sklerotherapie 467

Varizenverödung 562

Vektorkardiographie $1172^{*}$

Venendruck und Schock 1043

Venendruckmessung $1211^{*}$

Venenerkrankungen und Kompressionsverbände 73

Verbrennungskrankheiten $1063^{*}$

Verdauung des Kindes, Handbuch 1172

Vererbung der Hautleisten 969*

-, plasmatische $\mathbf{7 1 5}$

Verfolgungsspätschäden gynäkologischer Art 260

Vergiftung und Magenspülung 485

Verkehrsmedizin und Diabetes 697

Verkehrsunfall und Hals wirbelsäule $\mathbf{5 8 8}$

- und Steuerabsetzung 139

Venerologie, Handbuch 95

Veronal, Entgiftung 291

Versorgungsverbände, kommunale und Gesetz $48^{\circ}$

Verstärkerauskultation, Vorteile 829

Verteilungschromatographie und Barbiturate

Vertebralis-Insuffizienz, Chirurgie $66 \mathrm{R}$

Vestibularis und Streptomycin 1155

Verwaltungsgerichtsordnung $833^{*}$

Vinblastin und Choriokarzinom 68

Virämie nach Poliomyelitisimpfung $\mathbf{3 8 5}$

Viren und Autoimmun.

krankheiten 477

- und Embryo 1008

Virologie, medizinische und Fortschritte $96^{\circ}$

-, Praxis 1063*

Virusforschung und Leukämie $235^{\circ}$

Virushepatitis und Schwangerschaft 1118

Virusinfektion, experimentelle $46^{*}$

Virusinfektionen im Kindesalter $\mathbf{5 3}$

- des Menschen 423

Viruskrankheiten, Pathologie $328^{\circ}$

Viruspneumonien 276

Vitalkapazität 659

- und Rezteptorenblocker 146

- in der Praxis 725

Vitamin $B_{6}$ bei Cystathioninurie 189

- $B_{6}$ und Hydralazin 1137

- und Ovulationshemmer 1174

- $B_{12}$, Bestimmung und Chlorpromazin 425

Vitamin $B_{12}$ und Magenresektion 846

- $\mathrm{B}_{12}$, Mangel und Methylmalonsäureausscheidung 141

- C und Kohlenhydratstoffwechsel 107

- D und Herz-GefäßErkrankungen $\mathbf{8 8 1}$

- E, Mangel 833

- E bei Muskeldystrophie 115

- $K$ und Blutungsübel des Neugeborenen $\mathbf{8 0 0}$

- $\mathrm{K}$ und Gerinnung $\mathbf{3 1 7}$

Vitaminbestimmung, Methoden $876^{\circ}$

Vögel und Salmonellen $48^{\circ}$

Volvulus im Alter 953

Vorderwandinfarkt und $\mathrm{Q}$-Zacke 21

- und Thrombolyse $\mathbf{5 8 5}$

- EKG 982

Vorhofflimmern, Defibrillation 39

Vorhofscheidewanddefekte, Therapie $\mathbf{6 2 7}$

$\mathbf{W}$

Waaler-Rose-Test 220

Wärmeautoantikörper inkomplette $\mathbf{4 7 4}$

Wärmehaushalt $1212^{*}$

Warzenbehandlung durch Kosmetikerin 869 R

Wasserhaushalt $1212^{*}$

Wassermann-Reaktion und Mycoplasma pneumoniae 1015

Wasserstoffionen und Säure-Basen-Regulation 601

Weichteilverletzungen und Frakturen $668^{\circ}$

Werksangehörige, erkrankte und Werksarzt 722

Werksarzt und Werksangehörige $722 \mathrm{R}$

Werlhof, Immunologie und Klinik 1069

Werner-Syndrom und Insulinresistenz 973

Wiederbelebung und EEG 227 


\section{$\mathbf{X}$}

Xanthelasmen und Hypercholesterinämie 282

Xanthopsie $\mathbf{3 7 3}$

Xanthurensäure und Ovulationshemmer 1174 XO-Typen 1095

Xylose, Bestimmung 235* Xylosetest 562

$\mathbf{Y}$

${ }^{90}$ Yttrium und Nebennierenhyperplasie $\mathbf{1 7 7}$

\section{$\mathbf{Z}$}

Zeckenbiß und Encephalitis in Südwestdeutschland 1143
Zeckenstich und Erythema chronicum migrans 233

Zehen, Deformitäten $832 *$

Zeissler, Johannes $\$ 232$

Zeitschriften, neue 669

Zelle und Arzneimittel 294

- und Erdalkali-Ionen 96*

Zelle, Feinstrukturen 1212*

- und Molekularbiologie $379^{*}$

- und Vererbung 716

Zellelektrophorese $1007^{\circ}$

Zellen, Feinstruktur $611^{*}$

Zentralnervensystem und Anämie 673

- und biogene Amine $\mathbf{3 1 8}$

- und Herpes simplex 1184

Zentropil bei Epilepsie 127

- und Magen-DarmStörungen 1061

Zervikale Symptome nach Trauma 590

Zigarettenrauchen und Blasenkrebs 565
Zigarettenverbrauch und Eindämmung 1213

Zirkulationsstörungen und Infrarotthermographie 962

Zirrhose, Atiologie und Pathogenese $\mathbf{6 8 2}$

- cardiaque 735

- und Farbstofftest 214

- posthepatische und biliäre $\mathbf{7 3 4}$

Zollinger-EllisonSyndrom, Funktionsanalyse $\mathbf{5 9 7}$

_ _ - Pankreasadenom 639

Zoster duplex unilateralis 263, 998

Zucker, physiologisch wichtigste 467

Zuckerkranke, ABC 516

Zuckerkrankheit $1135^{*}$

-, Entstehung 696

- Probleme 749

Züchtigung bei Tonsillektomie $963 \mathrm{R}$
Zwangssterilisierung und Unfruchtbarkeit $\mathbf{2 6 1}$

Zwikker-Farbreaktion auf Barbiturate $\mathbf{8 1}$

Zwillinge und Myasthenia gravis 396

- und Knochenmarktransfusion 1064

Zwillingsforschung 831 *

Zyklusstörungen nach Verfolgungsspätschäden $\mathbf{2 6 0}$

Zystogramm beim Kind 931

Zystitis und Harnröhrendivertikel 514

Zytodiagnostik, gynäkologische 563*

-, Zytogenetik, Plasma 715

Zytologie des Duodenalsafts $\mathbf{5 4 8}$

Zytostatika und Choriokarzinomzelle 66

- nach Krebsoperationen 514

\section{Namenverzeichnis ${ }^{1}$}

A

Abel, M., Gießen 699

Abraham, R., Frankfurt a. M. 345

Ackermann, R., KölnLindenthal 1141

Adam, W., Tübingen 821

Adler, E., Düsseldorf 396

Aebi, H. $380^{\circ}$

Ahnefeld, F. W., Mainz 44

Alken, C. E. $364^{\circ}$

Allgöwer, M., Chur/ Schweiz 648

Ambrose, E. J. 1007*

Amelung, Düsseldorf 612*, 851, 1100.

Ammann, R., Zürich/ Schweiz 1172

Anders, W. 1006

Anger, G., Erfurt 429

Apitz, J., Göttingen $\mathbf{8 8 1}$

Arend, P., Marburg/Lahn 1180

Arnold, W., Hamburg 485

Aschoff, J., Erling-Andecks 610

Asshauer, E., Hamburg 606, 1003

Axhausen, W., Bremerhaven 1209

B

Babics, S. 1173*

Bader, G., Ludwigshafen 787*

Baensch, W. 667*

Baensch, W. E. 874*
Bálint, P. 1211*

Balzereit, F., Hamburg 485, 1194

Bargmann, Kiel 612*

Baron, D. N. 564*

Bartelheimer, Hamburg 1099*

Bauer, B., Freiburg i. Br. 1124

Baulieux, E. E. 284*

Baum, P., Mainz 660, 706

Baumann, E. 234*

v. Baumgarten, R. Göttingen 1061

Baumgartl, F., Düsseldorf 1208

Bay, V., Hamburg 1206

Bayer, O., Berlin 516

Bearn, A. G. $830^{\circ}$

Beck, K., Freiburg i. Br. 682

Becker, Göttingen $831^{*}$

Becker, Karlsruhe $140^{\circ}$

Becker, W., Bonn $46^{*}$

Beckmann, R. $45^{\circ}$

Beckmann, R., Freiburg i. Br. 108

Begemann, H., München-

Schwabing 1005

Beltz, L. Bonn 1177

Berchtold, R., Solothurn 1207

Berg, G., Erlangen 843

v. Berg, W., Göttingen $\mathbf{8 8 1}$

Berger, H., Freiburg $\mathbf{4 8 8}$

Berndt, H., Berlin-Buch $515^{*}$

Berghaus, H., Essen-

Holsterhausen 894

Berning, D., Freiburg i. Br. 569

Berning, H., Hamburg-

Barmbek 1090
Bertram, F. 516*

Beuren, A. J., Göttingen 881

Beyer, J., Frankfurt a. M. 1083

Biesalski, Mainz 328.

Biess, B., Münster/Westf. 250

Biglieri, E. G. 469*

Bläker, F., Hamburg 245

Bleuler, M. 968

Bloch, Berlin $1136^{\circ}$

Boch, J. 1006*

Bock, K. D. 920*

Bock, Tübingen $327^{\circ}, 516^{*}$, $786^{*}$

Bodechtel, G., München 673

Böckel, E. 876*

Böhle, E., Frankfurt a. M. 193, 1083

Böhler, L., Wien 1208

Böke, W., Münster/Westf. 505

Bolck, J. 668*

Bollinger, A., Zürich $874^{*}$

Bonin, Frankfurt $96^{\circ}, 423^{\circ}$, $1063^{*}$

Bonneville, M. A. 611*

Borchers, H.-G., München 673

Bottke, H. 140*

Bräutigam, Heidelberg $379^{\circ}$

Brand, G. 283*

Brandis, H., Göttingen 53, 153

Brånemark, P. I., Göteborg/ Schweden 961

Brauch, F. Bochum 593

Braun-Falco, O., Marburg/ Lahn 282

Brauner, München $727^{\circ}$
Braunsteiner, H., Innsbruck $140^{\circ}, 360,413,416,610$ $667^{\circ}, 750$

Brazier, M. A. B 516*

Brehm, G., Mainz 378

Brittinger, G., Homburg/ Saar 400

Brobeck, J. R. 1211*

Brosig, W., Berlin 326 $1007^{*}, 1019$

Browning, E. 668

Brück, Marburg 1211

Brücke, F., Wien 724, 1059

Brückner, R. 1062

Brühl, W. 1099*

Buchborn, Köln 1173*, 1212*

Budelmann, G., HamburgHarburg 1202

Bücherl, E. S., BerlinNeukölln 378

Büchner, M., Düsseldorf 1105

Bühler, H. R. 968*

Bühlmann, A., Zürich 82

Buentello-Malo, L., Freiburg 569

Büttner, H., Kiel 234, 235 $284^{*}, 562,784,829,1061$

Burck, H.-Chr. 1135

Burckhardt, W., Zürich 598

Bustamante, R. A. 516

C

Carstensen, Mülheim 1207

Cohn, W. E. $379^{\circ}$

Colmant, H. J. 423*

Cotta, H., Berlin 1208

Creutzfeldt, W., Göttingen 377,682

${ }^{\prime}$ Die fettgedruckten Zahlen bedeuten Orginalarbeiten, die mit ${ }^{\bullet}$ versehenen Buchbesprechungen. $R=$ Arzt und Recht. 
Crews, E. R. $1063^{\circ}$

Csiffáry, D., Budapest/ Ungarn 952

D

Dahl, P., Lübeck 211

Davidson, J. N. $379^{\circ}$

Daweke, H., Düsseldorf 973

Decker, H., Bonn-Venusberg 1158

Decker, München 469*

Defoe, D. 1063"

Delfino, A. J. 433

Demling, L., Bad Cannstatt 378

Denz, Ch., Freiburg 1183

Derra, Düsseldorf 627

Dettmar, H., Düsseldorf 514

Deucher, F., Aarau/ Schweiz 1001

Deupmann, F. J., Mülheim/ Ruhr 1035

van Deurzen, W. A. Dordrecht, Niederlande 303

Deutsch, E., Wien 186

Diederich, K. W., Göttingen 978

Diethelm, L. 468*

Ditschuneit, H., Frankfurt a. M. 814, 853, 1083

Dittrich, J. K., Marburg/L. 308

Dittrich, Marburg/Lahn 1205

Dix, V. W. 564*

Dobrowolski, J., Gliwice/ Polen 1121

Doctor X $727^{*}$

Döring, G. K., München 116, 260, 830*, 1005, 1047, 1098,1134

Dörr, W. M., Tübingen 168

Dorndorf, W., Frankfurt a. M.-Niederrad 662

Drews, J., New Haven/ Conn./USA 178

Drischel, H., Leipzig 1164

Drube, H. Ch., Kiel 283, 1061

Dubach, U. C., Basel 241

Düben, W. 564.

Düllo, H. 876

Dulce, H.-J., Berlin-

Dahlem 326

Dumermuth, G. $187^{*}$

E

Earl, A. S. $874^{*}$

Ebner, H., Gießen 376

Eckstein, H. B. 1210

Effert, S., Düsseldorf 39

Egenter, R. 424*

Ehlert, C. P. 1211

Ehlert, H., Darmstadt 422

Ehrhardt, H. 236*

Eichler, O. $46^{*}, 96^{\circ}$

Eisenreich, F. X., Gießen 1207

Emminger, E., Augsburg 1209
Emrich, D., Göttingen 101

Enders-Ruckle, G., Stuttgart 575

Engelmeier, Essen 424.

Epstein, M. A. 328*

Erbslöh, F., Gießen 45", 666, $1136^{\circ}$

Ernst, H., Berlin-Charlottenburg 333

Erpenbeck, R., München 634

Essbach, H. 1006

Esser, I., Saarbrücken $\mathbf{3 8 5}$

Etmer, F. 668.

Exner, G. $139^{\circ}$

Exner, Marburg 236*, 832*

Ey, W., Heidelberg 947

Eyer, H., München 919 1202

Eyermann, E. 833*

\section{$\mathbf{F}$}

Fankhauser, S., Bern 763

Farah, A. 46*, 96*

Fawcett, D. W. 1212*

Federlin, K., Frankfurt a. M. 814, 853

Fegeler, F., Münster/Westf. 250

Fellinger, K., Wien 1206

Fennell, D. I. 1173*

Fettweiss, E., Aachen $\mathbf{3 1 3}$

Feurstein, V. 1211*

Filippini, L., Zürich 359, 959

Finsterer, U., München 8

Fischer, A. W., Kiel 1211

Fischer, E., Zürich 411

Fischer, Freiburg i. Br. 920"

Fischer, J., Mainz 119

Fischer, L., Tübingen 138

Fleischhacker, Wien $46^{\circ}$

Fleischhauer, K., Hamburg 75, 318

Förster, H., München 95 , 106

Fontaine, R., Strassbourg 1207

Forell, M. M., München 634

Frahm, H., HamburgEppendorf 499

Frahm, M., Hamburg 81

Franke, Würzburg $379^{\circ}$

Frehner, H. U. 467*

Freudenberg, K., Berlin 137

Frey, R., Mainz 44, 140 $832^{*}$

Friedberg, Saarbrücken 564*

Frimmer, M., Gießen 33

Frisch, P. $327^{\circ}$

Fritze, E., Bochum 899

Fröhler, L. 833*

Frommhold, W. $667^{*}, 874^{*}$

Fuchs, E., Bad Lippspringe 904

Fuchsig, P., Wien 1207 , 1208

Fürstenberg, H., Mannheim 297

Fuhrmann, Heidelberg $1173^{*}$

Fuhrmann, W., Heidelberg 453

Funke, U., München 108
G

Gabler, G. $1135^{\circ}$

Gabrielsen, A. E. 47*

Gaca, Freiburg i. Br. 1007*

Gärtner, H., Kiel 803

Galli, H. 284*

Gauwerky, Hamburg $235^{\circ}$

Gehrmann, G., Düsseldorf $559,1069,1105$

Gercke, W. 876

Gerlach, J., Würzburg 1016

Gerok, W., Mainz 600

Giebel, M., Kassel 1209

Gibbels, E. 968*

Gigli, Irma, Frankfurt a. M. 819

Gillmann, Ludwigshafen $920^{\circ}$

Glauner, R. $667^{\circ}, 874^{\circ}$

Glocker, R. 234*

Gloor, F., Basel 241

Göb, A. 284*

Goerttler, K. 785*

Golab, W., Gliwice/Polen 1121

Good, R. A. 47*

Gottron, H. A., Mainz 95*, $1062^{\circ}$

Graf, K., Uppsala 911

Gramlich, F. R. 1210

Grant, L. 920*

Griesbach, Augsburg 283*

Griesser, G., Kiel 121

Grob, M., Zürich 1209

Gronemeyer, W., Bad Lippspringe 902

Gross, Frankfurt 1207

Gross, R., Köln 521

Gstirner, F. 876*

Günther, O., Frankfurt a. M. 87, 1197

Günther, R., Hamburg 1144

Günthner, W. 140*

Gütgemann, A., Bonn 1208

\section{H}

Haas, R., Freiburg i. Br. $385,423^{*}, 569$

Hacks, S. $787^{\circ}$

Haemmerli, U. P., Zürich 422

Hänze, S., Mainz 837

Hafter, E., Zürich 90, 139

Haid, H., Stuttgart $\mathbf{7 3}$

Haller, Göttingen 875*

Haller, J. 830*

Halmagyi, Mainz 140

Hammar, C. H., Bochum 899

Hangos, G., Budapest/ Ungarn 932

Hansen, W., Innsbruck 750

Harbers, Göttingen 379*, 517

Harder, H. J. 47*

Harms; H., Hamburg 658

Harper, H. A. 1062*

Haueisen, F., KasselWilhelmshöhe $232 \mathbf{R}$ $465 \mathrm{R}$

Hauser, W., BonnVenusberg 998

Hausser, R., Löwenstein 376

Hayhoe, F. G. J. 235*

Heberer, G., Köln 1207

Heckmann, U., Homburg/ Saar 66

Heckner, F., Einbeck 580, 978

Heffter, A. 46*, 96*

Hegemann, G., Erlangen 336

Hegglin, J., Chur 648

Hegglin, R. 423*, 874*

Heidler, S., Erfurt 429

Heilmeyer, L. 563*

Heinecker, R., Kassel 768

Heinemann, G., Frankfurt a. M. 814

Heinemann, G. W. 1100

Heinrich, F., Gießen 859

Heinrich, K. 467*

Heintz, R., Frankfurt a. M. 1074

Helbig, D., Köln-Riehl $\mathbf{5 7}$

Hellbrügge, Th., München 783

Helle, S., Berlin-Britz 263

Helpap, B., Marburg/L. 493

Helpap, E., Erlangen 493

Hemmer, R., Freiburg i. Br. 770, 867

Heni, F., Tübingen 174

Henne, G., Homburg/Saar 400

Hennemann, H. H., Mannheim-Waldhof 473

Henning, N., Erlangen $\mathbf{8 4 3}$

Henry, R. H. 424*

Henschler, Würzburg $668^{\circ}$

V. Herrath, Berlin 379

Herzog, H., Basel 725

Hess, Dortmund $1100^{\circ}$

Hess, R., Zürich 126

Hess, W., Zürich 660, 784

Hettinger, Mülheim/Ruhr $517^{*}$

Heubner, W. 46*, 96*

Heupke, W. $1135^{\circ}$

Heyck, Berlin 423*

Heymer, A., Bonn-Venusberg $138,235^{\circ}$

Hilger, M. L. 727

Hilmer, W., NürnbergErlangen 15

Hioco, D., Paris 1079

Hipp, E. $187^{\circ}, 284^{\circ}$

Hirche, $\mathrm{Hj}$., Düsseldorf 1

Hirschmann, E., ErlangenNürnberg 479, 822

Hodler, Bern $1007^{\circ}$

Höffler, D., Berlin 206

Hoen, Darmstadt 328*

Hoffineister, H. E. 1207

Hofmann, D., Frankfurt a. M. 348

Hofmann, G. G., München 753

Hohenfellner, R., Homburg/ Saar 930

Hohmann, Bergen 284*

Hoigné, R. $727^{\circ}$ 
Hornbostel, Hamburg 424*, 469

Hornof, Z. 1212

Hoschek, Stuttgart 139 $969^{\circ}$

Hossli, G., Zürich 29

Huber, C., Innsbruck $\mathbf{3 6 0}$ 416

Huber, H., Innsbruck $\mathbf{3 6 0}$, 413, 416

Hübener I, J. 515*

Hueck, A. 1063"

Ippen, Düsseldorf $380^{*}$, $4^{\circ}, 1063^{*}$

Irmer, W., Düsseldorf $\mathbf{4 3 3}$ 627, 1206

\section{$\mathbf{J}$}

Jacchia, E $1136^{\circ}$

Jakobovits, A. 831*

Jakovcic, S., Chicago 453

Janzen, R., Hamburg 1192

Jawetz, E. 469*

Jerusalem, F. 328*

Joch heim, K.-A. 968

Jörgensen, G., Göttingen 507, 881

Jonasch, E. 564"

Jung, A., Innsbruck $\mathbf{7 5 0}$

Junghanns, H., Frankfurt a. M. $421,564^{*}, 611$

Just, Heidelberg $48^{\circ}, 1212^{\circ}$

\section{$\mathbf{K}$}

Kabelitz $1006^{\circ}$

Käckell, M. Y., Göttingen 53,153

Käser, Frankfurt a. M. 188*

Kaeser, H. E. Basel $\mathbf{5 5 0}$

Kahlke, W., Heidelberg 26

Kaiser, B., Göttingen 881

Kaiser, E., Zürich 1205

Kaiser, H. 46.

Kaiser-Meinhardt, I. $327^{\circ}$

Kalkoff, K. W. Freiburg 919

Kaltenbach, M., Frankfurt a. M. 884

Kantner, Heidelberg $785^{\circ}$

Karlson, P., Marburg/Lahn $516^{\circ}, 564^{\circ}, 786^{\circ}, 920^{\circ}$, 1063

Kass, E. H. 1006

Kaufmann, H., Essen-

Holsterhausen 339, 894

Kaverina, N. N. $727^{\circ}$

Keiderling, W. 832*

Kelemen, E. $424^{\circ}$

Kelikian, H. $831^{\circ}$

Keller, H., Stuttgart 159 , 377

Keller, W. 563*

Kern, E., Freiburg 467

Keuth, U. 423"

Kielholz, Basel 421, 467*

Kienle, G., Frankfurt a. M. 444

Kikillus, B., Mainz 119

Kirchhoff, H., Göttingen 187, 1006*

Klausberger, E. M. 968

Kleihauer, E., Tübingen 1162 v. Klein-Wisenberg, A. Freiburg i. Br. 198

Klenk, E., Köln 280

Klepzig, H., Königstein i. Ts. $44,327 * 379 *, 725$

Klett, H., Marburg/Lahn 403

Klingler, Basel 328*

Klinner, W., München 8

Klosterhalfen, H., Hamburg 234, 1206

Kluthe, R., Freiburg i. Br. 536

Knight, J. 47*

Kober, P., Kiel 373

Koch, A., Münster 1212

Koch, H. 727*

Köberle, H., Tübingen 147

König, E., Homburg/Saar 400

König, P. A., Tübingen 467

Köppel, Gießen $875^{\circ}$

Körtge, P., Berlin 581, 978

Kohlhaas, M., Karlsruhe-

Durlach $41 R, 277$ R, 323

$R, 418 R, 783 R, 828 R$, $1057 \mathrm{R}$

Kohlmeyer, K., Gießen 699

Kole, E. A., Dordrecht, Niederlande $\mathbf{3 0 3}$

Koll, Göttingen 47*, $97^{\circ}$

Koller, F., Basel 1205

Kollmannsberger, A., München 673

Kollwitz, A.-A., BerlinCharlottenburg 1035

Korting, G. W., Mainz 233, 501, 1098

Kory, R. C. 516*

Kräubig, H. $1006^{*}$

Kranz, H. 467*

Kranz, Mainz 380*

Krauss, Freiburg i. Br. $468^{\circ}, 668^{\circ}$

Krayenbühl, H. 726*

Krayenbühl, Zürich $969^{\circ}$

Krech, U., St. Gallen/

Schweiz 1013

Kremer, K. 1207

Krentz, H., HamburgEppendorf 356, 596

Kreutz, F. H., Marburg/ Lahn 283

Kreuzer, A. 1212

Krokowski, E., Berlin 60

Krück, Homburg/Saar 284*

Krupp, M. A. 469*

Krzeminska, Lawkowicz, I $667^{\circ}$

Krzywanek, H. J., Frankfurt a. M. 193

Kühn, A. $1007^{\circ}$

Kühn, Gießen 139*

Kühn, Gießen 188*

Kühn, H. A., Gießen 466*

Kümmerle, F., Mainz 643

Künzer, W., Freiburg i. Br. 793, 1172*,1183

Künzer, Freiburg i. Br. $423^{\circ}$

Küpper, B., Köln-Lindenthal 1141

Kuhlendahl, H., Düsseldor 801, 1209

Kuhn, E., Heidelberg 947

Kunz, H. 283*

Kupferschmidt, H. G. $1137^{\circ}$

Kuschinsky, G., Mainz 233. $4^{\circ} 3^{\circ}, 516^{*}, 611^{\circ}, 727^{\circ}$,

$1004,1150,1211^{\circ}$

Kuttner, A. B. S. $328^{\circ}$
Laborit, H. 832*

Lamb, L. E. $1172^{\circ}$

Lamina, J., Frankfurt a. M. 848

andbeck, G. Hamburg 245

Landolt, H., Zürich 539

Lange, H. P., Frankfurt a. M. 254

Lange, K. 284*

Lange, M. $187^{\circ}, 284^{\circ}$

Langer, H. 1006*

Lasch, H. G., Gießen 859

Laubenthal, F. $327^{\circ}$

Lauber, H. L. 188*

Laubinger, G., Hamburg 1144

Lausberg, G., Gießen $\mathbf{1 1 0 9}$

Lawkowicz, W. 667"

Lehmann, Ch. 1210

Lejeune, Villach/Kärnten $1137 *$

Lembeck, F. 423

Lembeck, Tübingen $140^{\circ}$

Lendle, Göttingen $46^{*}, 325$ $611^{*}, 668^{*}$

Lenz, W., Münster/Westf. 1132

Leonhardt, Kiel 1135*

Letournel, E., Paris 1209

Letterer, Tübingen 283*, $328 *$

Leuthardt, F. 423*

Lieboid, R. $1100^{\circ}$

Liebscher, S., Dresden 256

Lindemann, K. 236*

Linder, F., Heidelberg 1206

Lindner, H., Hamburg 267. 1160

Link, R., Hamburg 1207

Linneweh, F 786*

Löffler, G., Homburg/Saar

Lohmann, R., Köln-Merheim 984

Longmire, W. D., Los Angeles 1210

van de Loo, J., Köln $\mathbf{5 8 1}$ 978

Loogen, Düsseldorf $327^{\circ}$

Loose, K. E., Itzehoe/ Holstein 812, 1207

Lorgé, M., Zürich 539

Lossen, Mainz 1136*

Lotz, W., Frankfurt a. M. 1074

Lüllmann, H. 611*, 727*

Lüth, P. 1173*

Luthe, W. $379^{\circ}$

Lutz, H., Heidelberg 1043

Lutzeyer, Aachen 1007*

Lydtin, H., München 145

\section{$\mathbf{M}$}

Maass, G., Freiburg i. Br. 1099

Macherauch, E. 234*

Mähr, G. Gießen 306

Mailloux, M., Casablanca/ Marokko 370

Majer, M., Freiburg i. Br. 385

Majer, M., Bern 1183

Mappes, G., Mainz 643, 1210

Margraf, O., Aschaffenburg 348

Markoff, N. G., Chur/ Schweiz 918

Markowitz, M. A. B. 328

Marongiu, F., Freiburg i. Br. 101, 198

Martin, H., Frankfurt a. M. 55

Martini, A., Marburg/Lahn 1180

Martini, G. A., Marburg/ Lahn 221, 1207

Martius, G., München 610

Marx, Marburg/Lahn 141, 876*

Masson, M., Homburg/Saar 400

Matussek, P. $424^{*}$

May, München 564*

Mayer, H., ErlangenNürnberg 919

McCluskey, R. T. 920*

McIntyre, N. $188^{*}$

Meigen, B., Freiburg i. Br.

Meinrenken, H. 188*

Menne, F. 284*

Mehnert, F., MünchenSchwabing 106

Mehnert, H., MünchenSchwabing $95,186^{\circ}, 326$, 467, 744, 873

Meier, J., München 145

Meites, S. 235"

Melani, F., Frankfurt a. M. 1083

Melnick, J. L. 96*

Mertz, D. P., Freiburg 529, 536, 541

Meyer-Rohn, J., Hamburg 282

Michel, Berlin 516

Miescher, G. I 1062*

Mikat, B. 283*

Minneker, C., Münster/

Westf. 434

Mitchell, J. S. 785*

Mitchell, R. S. 876*

Modde, H., St. Gallen/ Schweiz 1013

Moeschlin, S. 96*

Mohr, Heidelberg 1136"

Moll, W., Basel 219

Møller, K. O. 1211*

Morell, B., Bern 763

Morhard, K., Stuttgart 723 R

Mühler, E., Saarbrücken 1114

Müller, E., Bochum 588 
Neuhaus, G. A., Berlin 514

Neumann, P., Gießen 306

Nigst, H., Basel 234*, 1208

Nissen, R., Basel 283*, 622 $874^{*}$

Nöbel, B. 1063*

Nödl, F., Homburg/Saar 1093

Noelle, H., Gießen 1135

Noetzel, H. 328*

Nolte, R., Göttingen 53, 153

Nolting, S., Münster/Westf. 250

Nultsch, W. $726^{\circ}$

\section{O}

Obeck, V. 517*

Ober, K. G. 188*

Oberniedermayer, A. 1210

Obladen, H., Düsseldorf 433

Ody, R., Bochum 488

Oehme, J., Braunschweig 129

Oeff, Berlin $469^{\circ}$

Okrös, S. 969*

Oeser, H., Berlin-

Charlottenburg 333

Ohler, W. G. A., Mainz 119

Ohm, H. G., Düsseldorf 1035

Olsson, O. $468^{*}$

Opitz, H. 1172*

Orthner, H., Göttingen 984

von Osten, V., Freiburg i. Br. 108

Ott, V. R., Bad Nauheim 967

Otte, H.-J. 875*

Ottenjann, R., Erlangen 1045

Ottenjann, R., Stuttgart 1134

Otto, H. 516*

Otto, H., Münster/Westf. 934

$\mathbf{P}$

Pabst, K., Mainz 600, 706

Parlowitzki, I. H., Münster/ Westf. 1094

Pastinszky, I. $381^{*}$

Paul, J., Erlangen 389

Petrides, P., Duisburg 689

Petzoldt, D., Marburg/Lahn 909

Pezold, F. A., Berlin-

Zehlendorf 581, 978

Pfalz, R., Hamburg 409

Pfeiffer, Frankfurt a. M. 314, 468*, 1083

Pia, H. W., Gießen/Lahn 173,925

Piekarski, G. 1006*

Piening, O., Lübeck 211

Pierach, A., Mainz $\mathbf{8 3 7}$

Pincus, G. $874^{*}$

Pitzen, P. 236*

Poliwoda, H., Hannover 581, 978

Ponsold, Münsțer/Westf. $1172^{\circ}$
Porter, K. R. 611*

Pothe, H., Erfurt 1186

Praetorius, F., Berlin 581, 978

\section{$\mathbf{R}$}

Raab, W., Burlington/Vt./ USA 193

Ráczi I. 380*

Raper, K. B. $1173^{\circ}$

Rath, G., Göttingen 1134

Rathke, F.-W., Ludwigsburg $45,787^{*}$

Reich, Th., Zürich 135

Reiss, G., Karlsruhe $\mathbf{5 6 9}$

Reissigl, H. 140*

Remky, H. 1006*

Remmer, H., Tübingen 289

Remy, Bremen 235*

Remy, D., Bremen 1055

Rényi-Vámos, F. 1173*

Reploh, H. 875*

Rettig, Gießen 139*

Reubi, F., Bern 763

Richter, G. W. 328*

Robbers, Sigmaringen $1135^{*}$

Robel, P. 284*

Roberts, J. C. $612^{*}$

Rodenburg, R., Hannover 978

Rodewald, G., Hamburg 658

Röbbelen, G., Göttingen $\mathbf{7 1 5}$

Roemer, G. B., Hamburg $466,876^{*}, 967$

Röse, I. $1006^{\circ}$

Roschke, W., Bad Salzuflen 45, 830

Rossenbeck, H. G., Frankfurt a. M. 348, 363

Rotter, Frankfurt a. M. 468.

Rother, Kl., New York 551

de Rudder, B. ¥ 422*

Rügheimer, E., Erlangen 1210

Rütt, Würzburg 187*

Rusch, D., Bad Nauheim 967

S

Sachtleben, Homburg/Saar 229, 1007*

Sailer, S., Innsbruck $\mathbf{7 5 0}$

Sandritter, W., Gießen 376, 875*

Sarles, H. 139*

Schadewaldt, Düsseldorf $1063^{*}$

Scharfetter, Ch., Innsbruck 223

Schaudig, H., Erlangen 336

Scheibenreiter, S., Wien 216

Scheid, W., Köln-Lindenthal 1134, 1141

Scheid, W. 968*

Scheler, F., Göttingen 206

Schenck, W., Freiburg i. Br. 458

Scheppokat, K. D., Hamburg 1207

Schettler, G., Heidelberg $43,236^{*}, 612^{*}, 703,1133$

Schindler $1136^{*}$
Schinz, H. R., Zürich 135, $667^{*}, 874^{*}$

Schirmeister, J., Freiburg 726

Schlachetzki, J., Göttingen 448

Schlange, Hildburg, Göttingen 881

Schlöndorff, G., Bonn 555

Schmelcher, R., Karlsruhe $48^{\circ}, 91 \mathrm{R}, 365^{*}, 366^{\circ}, 380^{\circ}$, $416 \mathrm{R}, 417 \mathrm{R}, 463 \mathrm{R}$, $464 \mathrm{R}, 509^{\circ}, 510^{*}, 558 \mathrm{R}$, $559 R, 608 R, 665 R, 66 R$ $721 \mathrm{R}, 728^{\circ}, 780 \mathrm{R}, 787 \mathrm{R}$ $826 R, 833 R, 869 R, 872 R$, $876^{*}, 963 \mathrm{R}, 1064^{*}, 1150^{*}$, $1169 \mathrm{R}, 1171 \mathrm{R}, 1212^{*}$

Schmid, A., Zürich 539

Schmid, F. 1172

Schmid, L. 1212

Schmidt, O. P. $140^{*}$

Schmidtke, A. 968*

Schmitt, A., Saarlouis $\mathbf{4 4 4}$

Schmutzler, R., Basel 269, 297, 316, 381, 978

Schneider, B., Hannover 978

Schneider, C., HamburgEppendorf 454, 819, 1122

Schneider, K. $785^{*}$

Schneider, Würzburg 563*

Schneweis, K. E., Göttingen 53,153

Schöffling, K., Frankfurt a. M. 694,1083

Schoen, R. 423.

Schönfeld, W. 95

Schoop, G., Frankfurt a. M. 848

Schoop, H. D., Frankfurt a. M. 891

Schoop, W., Freiburg i. Br. 784

Schrader, A., MünchenHarlaching 1171

Schrader, K. E., Gießen 306

Schröder, E., Mülheim/Ruhr 1035

Schubert, G. E., Tübingen 147

Schubert, J. C. F., Frankfurt a. M. 55

Schulte, Tübingen $968^{\circ}$

Schulten, H. K., KölnFlittard 521

Schultze, R., Mainz 1211

Schumacher, H., Freiburg i. Br. 385

Schumacher, J., Freiburg i. Br. 419

Schwalb, H., München 873

Schwarz, K., München $\mathbf{7 5 3}$

Schwiegh, H. 423*

Scriba, P. C., München 753

Seeliger, Würzburg $48^{\circ}$, $787^{*}, 1173^{*}$

Seidel, W., München 8

Seidenfaden, I. 968*

Seifert, G., Hamburg 95, 778, 1208

Seifert, K., Kiel 321

Seige, K. 187*

Selye, H. $140^{*}$

Senning, $\AA$., Zürich 641 1206

Settele, R. $48^{*}$

Shapiro, M. 1006"

Sherlock, S. 188*

Siebelist, I. $1063^{\circ}$

Siebert-Hilger $727^{*}$

Siegal, L. J. 1172*

Siegl, J. 235

Sigg, K., Binningen/Basel 467,562

Sinapius, D., Göttingen 53, 153

Smolka, H. 563.

Sökeland, J., Homburg/ Saar 930

Solomons, B. 1099 .

Sommerkamp, H., Marburg a. d. Lahn 403

Soost, H. J. 563"

Spaethe, R., Münster/Westf. 934

Spielmann, W., Frankfurt a. M. 186, 667, 1006*, 1172

Spiess, H., Göttingen 575, 1171

Spoerri, Th. $379^{\circ}$

Spohn, K., Karlsruhe 1208

Sprössig, M., Erfurt 429

Staemmler, Kiel 831*

Staib, W. H. 515*

Stammler, A. 968

Starke, G. $1063^{\circ}$

Staubesand, Freiburg i. Br. $920^{\circ}$

Staudinger, Gießen $380^{*}$ 920*

Stegemann, I., Göttingen 206

Steigleder, G. K., KölnLindenthal 96*, 398

Steinacker, H. G., Homburg/Saar $\mathbf{4 0 0}$

Steinberg, G. A. $830^{*}$

Stelzner, F., Hamburg 1207

Stephan, Erlangen-Nürnberg 187*

Stickl, H., München $\mathbf{4 5 5}$

Stille, W., Frankfurt a. M. 891

Stoeckenius, M., Hamburg 125

Stoermer, J., Göttingen 881

Storck, H. 1062*

Straub, E., Homburg/Saar 930

Straus, R. 612*

Strehler, B. L. 283*

Streicher, H.-J., Marburg/ Lahn 991

Streiff, B. $920^{*}$

Strnad, F. $468^{\circ}$

Ström, G., Uppsala 911

Strohecker, Darmstadt $876^{*}$

Sturm, A., WuppertalBarmen 94 
Thaler, $\mathrm{H}$., Wien 733

Thalhammer, O., Wien 216. 1006*

Thierfelder $1099^{\circ}$

Thoenes, W. 468*

Thurn, P., Bonn 1177

Thurzó, R., Budapest/ Ungarn 952

Töndury, G. 378*

Tranekjer, S., Homburg/ Saar 66

Truckenbrodt, H. 422* 479

Trüb, C. L. P., DüsseldorfOberkassel $133 \mathbf{R}$

Tsagaris, T. J. 516*

Tünte, W. $1100^{*}$

Turner, R. A. $140^{*}$

\section{$\mathbf{U}$}

Uehlinger, E. $667^{\circ}$

Uehlinger, F. 874

Ulmer, W. T., Bochum 514

Uthgenannt, H., Lübeck 211, 437

\section{V}

Veihelmann, D., Freiburg i. Br. 536

Vieten, H. $468^{\circ}$

Vivell, O. 282, 423*, 569

Vogelsang, H., Gießen 173

Vogt, D., München 1005

Vorherr, H., Frankfurt

a. M. 345
Vorlaender, Bonn $47^{\circ}$

Vorlaender, K. O., Bonn 233

Vossschulte, K., Gießen 1208

\section{W}

Wacker, A., Frankfurt a. M. 178

Wagener, H., Heidelberg 861

Waibel, P., Basel 23

Walther-Büel, H. 379

Walton, J. N. $1136^{\circ}$

Wannemacher, E., Münster 967

Waterston, D. J., London 1209

Weber, G. $1100^{*}$

Weber, G., Nürnberg 326

de Weck, A. L., Bern 999

Wegmann, T., St. Gallen 711, 713

Weicker, Bonn 1100

Weinges, K. F., Homburg/ Saar 773

Weinreich, J., Lübeck $235^{\circ}$ 437

Weinschenk, C., Marburg/ L. 461

Weissbecker, L., Kiel 726, 832*, 1202

Weitzel, G. 515*

Wellauer, J. 667*, 874*

Weller, Freiburg 234*, 564*, 668
Wellmann, K. F., Brooklyn New York 131, 1211*

Wellmann, New York 727

Welte, E., Mönchengladbach 422

Wendt, Marburg 969*

Werner, H. 1006*

Werner, K., Bochum 899

Werner, M., Pinneberg 95

Wesle, H., Freiburg i. Br. 536

Wetzels, E., Düsseldorf 562

Weyer, F., Hamburg 186

Weyrauch, H. M. 564"

Widmer, L. K., Basel 863

Wieck, H. H. 968.

Wieck, Köln 785*

Wiemers, K., Freiburg 1210

Wiener, A. S. $1006^{*}$

Wilkinson, J. H. 612*

Willi, J. 968

Windorfer, A., Erlangen 422*, 424*, 563*, 786* $1006^{*}$

Winter, C. C. $469^{\circ}$

Wiskott, A. 563*

Witt, A. N., Berlin 1208

Witte, S., Erlangen 548, 1205

Witzleb, W. Erfurt 429

Wöckel, W., Erfurt 273

Wolf, H., Góttingen $\mathbf{5 7 5}$

Wolf, Heidelberg 236*

Wolff, H. P., Homburg/ Saar 738

Wolstenholme, E. W. 47*
Wüst, H., Erlangen 843

Wullstein, H., Würzburg 1016

Wunderlich, P., Dresden 256

Wustrow, F. 46

Wuth, K., Berlin 139, 366 R, 609 R, 722

\section{$\mathbf{Y}$}

Yamato, W. S. 1212

Yasargil, M. G. 726*

Yi-Yung Hsia, D., Chicago 453

\section{Z}

Zach, J., Köln-Merheim 521

Zdansky, Basel 667* 874*

Zehm, S., Würzburg 1016

Zeitler, G., Erlangen $\mathbf{8 4 3}$

Zekorn, D., Frankfurt a. M. 581, 978

Zenker, R., München 8, 283*, 634

Zinke, R., Staupitz/Spreewald 327

Zöllner, N., München 145 $515^{*}, 1171$

Zülch, Köln 726*

Zukschwerdt, L., HamburgEppendorf 617, 1205

Zuppinger, A. 468

Zweifach, B. W, 920

\section{Personalnachrichten}

Berufungen, Lehraufträge, Ernennungen

Albrecht, H., Hamburg 834

Althoff, H., Kiel 189

Amelung, D., Düsseldorf 189

Bammer, H., Würzburg 330

Baitsch, H., Freiburg 50

Barbey, K., Tübingen 142

Bauer, K. M. Tübingen 426

v. Baumgarten, R.,

Göttingen 565

Becke, M., Heidelberg 470

Berg, St., Göttingen 97 1009

Bergeder, H.-D., Bonn 728

Berger, Z., Basel 613

Bethge, J., Hamburg 722

Blaschko, H., Heidelberg 286

Borneff, J., Mainz 729

Brandt, A., Halle/Saale 285

Braun-Falco, O., Marburg 613

Brehm, H., Frankfurt 517

Brendel, W., München 50

Bretschneider, H. J., Köln 729

Brück, K., Marburg 381

Buchborn, E., Köln 1065

Buchborn, E., München 237

Bushe, K.-A., Göttingen 565
Carstensen, G., Würzburg 1065

Cotta, H., Berlin 237

Cremer, H. D., Gießen 189

Daubenspeck, K., Köln 1009

Demling, L., ErlangenNürnberg 728

Demling, L., Heidelberg 329

Dengler, J., Heidelberg 286

Dodt, E., Gießen 1174

Dohrmann, R., Berlin 669

Donat, K., Hamburg 1009

Eckel, W., Münster 426

Eder, M., München 1065

Effenberger, E., Hamburg 922

Effert, S., Düsseldorf 1174

Eggers, H. J., Gießen 1174

Egli, H., Bonn 728

Ehlers, P., Heidelberg 330

Eickhoff, H., Münster 614 , 1214

Engelmeier, M.-P., Essen

Erdmann, K. D., Kiel 1138

Erdmann, W.-D., Göttingen 518

Franke, J., Hamburg 922

Frick, W., Erlangen-Nürnberg 1214

Friedberg, K. D., Göttingen 565

Friedberg V., Mainz 1138
Friederiszick, F.-K., Mainz 878

Fröhlich, E., Tübingen 729

Fromm, E., Hamburg 518

Frommhold, W., Berlin 470

Gänshirt, H., Essen 50

Gahlen, W., Düsseldorf 669

Gassner, E., Bonn 1214

Gauer, O. H., Berlin 1214

Georgii, A., München 50

Gerlach, E., Freiburg 285

Gersmeyer, E. F., Mainz 286

Göpfert, H., Freiburg 1009

Göing, H., Frankfurt 329

Goslar, H. G., Bonn 613

Gottschalk, A., Tübingen 1215

Gregorczyk, K., Essen 1009

Gremmel, H., Düsseldorf 189

Gremmel, H., Kiel 613

Grewe, H.-E., Düsseldorf 669

Grunze, H., Berlin 1064

Gusek, W., Hamburg 1009

Haas, H., Heidelberg 877

Haberich, F.-J., Berlin 921

Habermann, E., Gießen 425

Häfner, H., Heidelberg 1175

Hager, H., Gießen 1174
Halbach, H., München 50

Hallen, O., Heidelberg 330

Hamm, J., Marburg 789

Hammerstein, J., Berlin 921

v. Harnack, G.-A., Hamburg 470

Hasselbach, W., Heidelberg 729

Haug, H., Göttingen 1009

Haunfelder, D., Sacrbrücken 470,1215

Hausser, E., Hamburg 237 , 426

Hedinger, Ch., Zürich 237

Heine, F., Münster 1215

Heintzen, P., Kiel 613

Heipertz, W., Heidelberg 286

Helbing, W., Halle/Saale 285

Hemmer, R. Freiburg 425

Henatsch, H. D., Göttingen 922

Henckel, W., Göttingen 565

Herre, W., Kiel 1138

Hertz, C.-W., Kiel 613

Hess, W., Basel 613

Heyck, H., Berlin 237

Heyden, S., Zürich 878

Hirsch, H., Köln 729

Höhne, G., Hamburg 518

Hörder, M., Lübeck 141 
Hoffmann, K., Essen 50 Hoffmann-Berling, H., Heidelberg 729

Hollwich, F., Münster 1009

Hopf, A., Heidelberg 877

Horatz, K., Hamburg 613

Hornbostel, H., Hamburg 189

Hort, W., Göttingen 97

Hossli, G., Zürich 237

Huth, E., Heidelberg 426

Ihm, P., Marburg 1214

Imhäuser, G., Köln 729

Jaeger, W., Heidelberg 50

Jensen, H.-P., Würzburg 729

Jusatz, H., Heidelberg 1175

Kanzow, U., Köln 1009

Katner, W., Köln 729

Kautzky, R., Hamburg 922

Kielholz, P., Basel 97

Kienitz, M., Münster 922

Kisker, H. P., Heidelberg 286, 878

Klein, F., Münster 1214

Kisker, K. P., Hannover 1101

Klingmüller, G., Würzburg 1065

Klosterhalfen, H., Hamburg 789

Kluge, E., Mainz 1138

Knapp, W., Bern 1137

König, A., Göttingen 518

Koepchen, H.-P., Göttingen 97

v. Kügelgen, A., Kiel 729

Künkel, H. A., Hamburg 922

Kunert, W., Bonn 970

Kuttig, H., Heidelberg 1065

Lautenbach, E., Bonn 728

Lehmann, H. J., Kiel 613

Lehnhardt, E., Hamburg 877

Leibbrand, W., München 1138

Lennert, K., Kiel 729, 1175

Leuner, H., Göttingen 97

Liebermeister, K., Frankfurt 517

Löffler, H., Basel 285

Löwnau, H. W., Kiel 330

Lorenz, W., Berlin 1064

Ludes, H. Köln 1009

Lühr, K., Göttingen 565, 1137

Lutzeyer, W., Würzburg 565

Marx, H.-H., Marburg 381

Meesmann, W., Essen 709

Meesmann, W., Göttingen 565

Meyer, J.-E., Göttingen 565

Meyer-Burgdorff, G., Kiel 381

Minnigerode, B., Göttingen 518

Mohr, H.-J., Münster 426

Mosler, K.H., Würzburg 566

Müller, H.-A., Würzburg 566

Müller, W., Köln 1009

Musshoff, K., Freiburg 285

Neubauer, H., Marburg 729

Oberniedermayr, A. München 670

Oehlert, W., Freiburg 285

Oksche, A., Gießen 1174

Pauly, H., Frankfurt 517
Petrilowitsch, N., Mainz 1102

Pfeiffer, E. F., Frankfurt 1064

Pflanz, M., Gießen 426

Pliess, G., Hamburg 285

Poppe, H., Göttingen 97

Rath, G., Göttingen 97

Rathke, F., Heidelberg 97

Reifferscheid, M., Bonn 728

Renker, U., Halle/Saale 285

Riecker, G., München 426

Ritter, U., Hamburg 97, 426

Ritze. H., Hamburg 922

Rodeck, G., Marburg 878

Rohrbach, H., Mainz 613

Rosenthal, A., Marburg 789

Rotthoff, G., Berlin 237

Rübe, W., Berlin 921

Rügheimer, E., ErlangenNürnberg 833

Sandkühler, St., Heidelberg 286

Sartorius, H., Freiburg 922

Schauer, A., München 670

Schedel, F., München 1065

Scheiffarth, F., Erlangen-

Nürnberg 833

Schellong, G., Münster 1215

Schermuly, W., Marburg 729

Schlegel, K. F., Köln 189

Schliack, H., Berlin 613

Schlüssel, H., Köln 1009, 1175

Schmidt, J., Erlangen-Nürnberg 1214

Schmidt, R., Heidelberg 1065

Schmidt-Matthiesen, H., Göttingen 1064

Schmiedt, E., München 834

Schmier, J., Heidelberg 426

Schneider, W., Tübingen 518

Schober, K. L., Halle/

Saale 237

Schober, R., Saarbrücken 1175

Schönenberg, H., Aachen 1214

Schreck, E., Erlangen-

Nürnberg 49

Schreier, K., Heidelberg 1138

Schroeder, W., Frankfurt 1064

Schüle, H., Erlangen-Nürn berg 49

Schulz, H., Düsseldorf 189

Schwarz, W., Berlin 425

Schwarzacher, H. G. Gießen 426

Schwenzer, N., Würzburg 566

Semisch, Hamburg 1009

Siede, W., Frankfurt 189, 1214

Sigel, A., Erlangen-Nürnberg 833

Spielmann, W., Frankfurt 1064

Steinbrecher, W., Essen 789

Stellmach, R. K., Düsseldorf 669

Stender, H.-St., Hannover 1009

Stier, A., Würzburg 566
Stoffel, W., Köln 1214

Strnad, F., Berlin 1064

Thoelen, H., Basel 613

Thofern, E., Göttingen 789

Thorban, W., Gießen 565

Thorn, W., Hamburg 1009

Tiwisina, Th., Münster 614

Truss, F., Göttingen 97

Uhlmann, G., Hamburg 518

Umbach, W., Freiburg 1137

Voth, H., Göttingen 97

Walser, A., Basel 613

Weibel, E. R., Zürich 190

Weinland, Helene,

Erlangen-Nürnberg 833

Werner, E., Berlin 921

Witt, H. J., Göttingen 922 1137

Wolf, H., Göttingen 1137

Wolf-Heidegger, G., Basel 97

Wrba, H., Heidelberg 518, 1102

Würterle, A., Marburg 789

Wundt, W., Tübingen 426

Zimmermann, W., Saarbrücken 1009

Zollinger, H.-U., Freiburg 517

\section{Habilitationen,}

Umhabilitationen,

Dozenturen

Anacker, H., München 1138

Aurand, K., Berlin 921

Balzer, H., Frankfurt 517

Baust, W., Heidelberg 1175

Beck, H., Erlangen-Nürnberg 1214

Bergleiter, R., Freiburg 729

Bianchi, L., Freiburg 613

Bockendahl, H., Saarbrücken 1009

Bolte, J., Köln 922

Bühlmeyer, K., München 426

Büttner, W., Gießen 565

Busch, M., Freiburg 613

Castano-Almendral, A. Göttingen 565

Cerletti, A., Basel 970

Deicher, H., Hannover 729

v. Deimling, O., Freiburg 613

Delbrück, A., Hannover 729

Denden, A., Göttingen 565

Devens, K., München 670

Dost, K., Freiburg 613

Emmrich, J., Göttingen 565

Englhardt, A., Düsseldorf 189

Engelhorn, R., Freiburg 1009

Falke, D., Mainz 729

Fiehring, H., Erfurt 1137

Finke, J., Tübingen 142

Franke, D., Heidelberg 613

Frenzel, H., Hamburg 426

Freyberger, H., Hamburg 426

Götze, H., Hamburg 426

Gosepath, J., Mainz 922

Gropp, H., Freiburg 729

Grünberg, H., Würzburg 729

Günther, Th., Berlin 921

Haas, H. G., Basel 970

Hallermann, W., Kiel 613

Hammersen, F., Freiburg 1174

Hanschke, H. J., Saarbrücken 470

Harndt, R., ErlangenNürnberg 49

Heidland, A., Würzburg 922

Heyden, S., Zürich 878

Hickl, E.-J., Essen 789

Höer, P., Saarbrücken 565

Hoferichter, J., Erlangen-

Nürnberg 49

Hoffmann, D., Hamburg 922

Holzmann, H., Mainz 922

Horster, F. A., Düsseldorf 189

Hübner, K., Frankfurt 237

Jaeger, J., Heidelberg 1102

Jurna, I., Saarbrücken 470

Kief, H., Frankfurt 237

Klaschka, F., Berlin 237

Kleibel, F., Heidelberg 518

Klemm, D., Freiburg 613

Klepzig, H., Freiburg 518

Kobes, L., Erlangen-Nürnberg 1214

Koburg, E., Düsseldorf 189

Königk, E., Hamburg 922

Kreuscher, H., Mainz 922

Kriegel, H., Freiburg 613

Kristen, K., Heidelberg 565

Langer, E., München 1065

Lieb, G. 729

Liefländer, M., Göttingen 565

Löhr, E., Essen 789

Marx, H., Mainz 922

Mayer, W., Tübingen 729

Meier-Ruge, W., Basel 189

Mestwerdt, G., Hamburg 922

Micke, H., Göttingen 97

Motsch, A., Freiburg 613

Müller, H.-A., Würzburg 330

Müller, H. J., Frankfurt 237

Müller, W., Erfurt 1137

Muth, H., Hamburg 922 
Suchenwirth, R., Lübeck 98 Tarnowski, W., Hamburg 922

Tölle, R., Tübingen 142

Tönnis, D. Berlin 921

Toussaint, W., Mainz 922

Träger, K., München 1065

Trautschold, I., München 426

Vogt, A., Freiburg 329

Vonderschmitt, H., Frankfurt 517

Voss, D., Tübingen 729

Wachsmann, F., ErlangenNürnberg 49

Wachsmuth, E. D., Frankfurt 517

Wagner, D., Freiburg 329

Waldeck, F., Mainz 922

Walter, K., Bonn 728

Weber, E., Heidelberg 518

Wechsler, W., Köln 922

Wenke, H., Göttingen 565

Wiemer, W., Bochum 1137

Winne, D., Tübingen 729

Willmanns, W., Tübingen 729

Wigand, M. E., Würzburg 729

Korrespondierendes Mit-

glied, Gesellschaften, Auszeichnungen

Amelung, W., Frankfurt 517

Ammon, R., Saarbrücken 470

Bärtschi-Rochaise, W., Bern 237

Beck, J., Erlangen-Nürnberg 517

Becker, J., Heidelberg 1065

Bischoff, P., Hamburg 1137

Brednow, W., Jena 729

Brügger, H., Tübingen 142

Butenandt, A., München 50, 470

Degenhardt, K.-H., Frankfurt 237

Derra, E., Düsseldorf 189, 669

Doerr, W., Heidelberg 1175

Flemming, K., Freiburg 1214

Frahm, H., Hamburg 922

Franceschetti, A., Genf 237

Frik, W., Erlangen-Nürnberg 517

Fromm, E., Hamburg 1137

Geissendörfer, R., Frankfurt 517

Götz, H., Essen 613

Gottron, H., Tübingen 565

Haberlandt, W. F., Tübingen 426

Harders, H., Hamburg 1065

Harmsen, H., Hamburg 426,729

Haunfelder, D., Saarbrücken 426

Hepp, O., Münster 613

Herrlinger, R., Kiel 426

Heuck, F., Kiel 426

Hirschmann, J., Tübingen 1215
Hoff, F., Frankfurt 425, 877,1214

Hollwich, F., Münster 614

Horstmann, E., Hamburg 729

Joppich, G., Göttingen 565

Junghanns, H., Frankfurt 237

Jusatz, H., Heidelberg 1175

Käser, O., Frankfurt 237

Klose, F., Kiel 426

Köttgen, U., Mainz 1214

Krayenbühl, H., Zürich 237

Lammers, Th., Mainz 1214

Langen, D., Mainz 729

Langendorff, H., Freiburg 1214

Langendorff, H., Freiburg 1137

Lettré, H., Heidelberg 237

Linder, F., Heidelberg 518, 1175

Loew, F., Saarbrücken 1009

Lynen, F., München 670

Mark, R. E., Münster 1009

Markus, B., Göttingen 565

Martini, G. A., Marburg 189

Meissner, G., Hamburg 518

Muth, H., Saarbrücken 565

Nissen, R., Basel 425

Nowakowski, H., Hamburg 729,922

Pribilla, W., Berlin 237

Schadewaldt, H., Düsseldorf 49

Schettler, G., Heidelberg 285

Schimpf, A., Saarbrücken 1215

Schinz, H. R., Zürich 142

Schneider, W., Tübingen 142, 286

Schnyder, U. W., Heidelberg 613

Schober, R., Saarbrücken 330

Schümann, H.-J., Essen 425

Schulte, W., Tübingen 381

Senning, A., Zürich 237

Steinhardt, G., ErlangenNürnberg 49

Ströder, J., Würzburg 729

Swart, B., Hamburg 729

Valentin, B., Rio de Janeiro 1102

Wagner, G., Heidelberg 426

Wagner, H., Münster 614

Weber, G., Mainz 98

Wetterer, E., ErlangenNürnberg 877

Witt, A. N., Berlin 237

Zenker, R., München 670

Zindler, M., Düsseldorf 189

Zülch, K. J., Köln 922

\section{Entpflichtungen}

Deuticke, H. J., Göttingen 922

Herrmann, W., Essen 789

Kolle, K., München 670

Leicher, H., Mainz 1138

May, F., München 1065

Mörl, F., Halle/Saale 237

Mueller, B., Heidelberg 1101
Rauh, W., Gießen 425

Stolze, M., Halle/Saale 237

Wannenmacher, Münster 1009

Weber, G., München 1065

Wiskott, A., München 1065

\section{Geburtstage}

Ackerknecht, E.-H., Zürich 1009

Amsler, M., Zürich 286

Asperger, H., Wien 330

Bargmann, W., Kiel 141

Beck, J., Erlangen 788

Becker, H., Münster 834

Böger, A., Mainz 470

Boller, R., Wien 1065

Brednow, W. Jena 286

Bredt, H., Mainz 189

Derra, E., Düsseldorf 425

Engelking, E., Heidelberg 834

Erlacher, J., Wien 381

Eyer, H., München 1175

Falk, P., Homburg/Saar 142

Froboese, C., Berlin 833

Grüneberg, Th., Halle/ Saale 426

Haas, G., Gießen 789

Hallermann, W., Kiel 470

Harndt, E., Berlin 141

Held, E., Zürich 566

Herbst, R., Graz 877

Herzberg, K., Frankfurt 833

Hess, W. R., Zürich 470

Hoff, F. Frankfurt 729

Holler, G., Wien 1138

Holstein, E., Berlin 922

Huber, P., Innsbruck 970

Jacobshagen, E., Marburg 878

Jaeger, F. H., Mainz 286

Janssen, S., Freiburg 285

Janz, H.-W., Hamburg 1174

Jores, A., Hamburg 237

Kemkes, B., Gießen 425

Kielleuthner, L., München 729

Knake, E., Berlin 1064

Knoflach, J. G., Wien 789

Koelsch, F., Erlangen-Nürnberg 1214

Koller, F. Basel 788

Kramer, K., München 729, 922,1102

Kranz, H., Mainz 141

Kühnau, J., Hamburg 922

Lampé, A. E., München 237

Leibbrand, W., München 142

Liljestrand, G., Stockholm 730

Lippelt, H., Hamburg 50

Matras, A., Wien 1138

du Mesnil de Rochemont R., Marburg 970

Meyer, W., Göttingen 613

Meyer zu Hörste, Münster 237

Mooser, H., Zürich 834

Niederwieser, V., Inns-

bruck 98

Niekau, B., Tübingen 565

Norpoth, L., Essen 670

Paschke, H., Erlangen 1174

Pitzen, P., Münster 1138

Perwitzschky, R., Münster

Prigge, R., Frankfurt 729

Pschyrembel, W., Berlin 285

Pulewka, P., Tübingen 286

Reinmöller, M., Rostock 878

Riehm, W., Münster 98

Rominger, E., Kiel 834

Roth, K., Darmstadt 142

Ruge, H., Kiel 381

Sarre, H., Freiburg 565

Schaumann, O., Innsbruck 670

Schmidt, B., Berlin 970

Schneider, R., Graz 1137

Schönholzer, G., Bern 728

Schoop, G. Frankfurt 877

Seiser, A., Erlangen 789

Singer, L., München 922

Solé, A. Wien 970

Staehelin, J. E., Basel 1064

Steudel, J., Bonn 425

Stöhr, Ph., Bonn 669

Sturm, A., Düsseldorf 1174

Suckow, J., Dresden 613

Sylla, A., Cottbus 471

Thoenes, F., Rostock 50

Töndury, G., Zürich 470

Traub, E., Tübingen 1175

Trautwein, K., Freiburg 470

Ubelhör, R., Wien 1215

Uebermuth, H., Leipzig 98

Waldeyer, A., Berlin 425

Weber, H.-H., Heidelberg 1138

Weitz, W., Hamburg 834

Wiedmann, A. Wien 670

Wittmaack, K., Hamburg 97

Wolfram, St., Wien 614

Zurukzoglu, St., Bern 613

Todesfälle

Bingel, K., Heidelberg 238

Binswanger, L., Kreuzlingen 382

Bürger, M., Leipzig 382

Diepgen, P., Mainz 238

Doepfner, R., Bonn 238

Dogliotti, A. M. Turin 1175

Edwards, Ph., Atlanta/USA 1138

Franke, H., München 382

Freudenberg, K., Berlin 238

Frornme, A., Dresden 1010

Heiss, J., München 98

Kehrer, F. A., Münster 789

Lindemann, K., Heidelberg 


\title{
Vergleich klinischer Methoden zur Erfassung des latenten Diabetes mellitus*1
}

\author{
Von P. C. Scriba, K. Schwarz und G. G. Hofmann
}

Aus der II. Medizinischen Klinik der Universität München (Direktor: Prof. Dr. Dr. G. Bodechtel)

Die Einteilung des Diabetes mellitus in verschiedene Stadien hat sich klinisch bewährt. Man unterscheidet heute den manifesten Diabetes mellitus, der mit erhöhten Blutzuckerwerten und Glykosurie einhergeht, von dem latenten Diabetes mellitus, welcher durch den pathologischen Ausfall bestimmter Belastungsmethoden gekennzeichnet ist und bei dem noch keine Glykosurie besteht. Ferner grenzt man eine Gruppe von Patienten mit dem sogenann-

- Professor Dr. W. Büngeler zum 65. Geburtstag.

1 Mit Unterstützung der Deutschen Forschungsgemeinschaft. ten Prädiabetes ab. Bei diesen Patienten fallen die Glucosebelastungstests normal aus; in ihrer Verwandtschaft, beispielsweise bei den Eltern, oder bei eineiigen Zwillingen ist nach der Definition ein Diabetes mellitus bekannt $(1,32,33,49)$.

Eine Reihe von Belastungsmethoden zur Erfassung des latenten Diabetes mellitus hat sich klinisch bewährt, in den USA führt man in erster Linie eine einfache orale Glucosebelastung durch, in Deutschland wird vielerorts die doppelte orale Glucosebelastung nach Staub-Traugott vorgezogen (2). Ferner kennt man seit Jahren eine intravenöse Glucosebe- 
lastung, die durch Berechnung des sogenannten Glucose-Assimilationskoeffizienten $\mathrm{k}_{\mathrm{G}}$ eine Erfassung der Glucosetoleranz ermöglicht (3-9). In der vorliegenden Arbeit berichten wir über unsere Erfahrungen mit diesen Formen der Glucosebelastung zur Erfassung des latenten Diabetes mellitus. Wir haben ferner in Analogie zum .Staub-Traugott-Versuch eine doppelte intravenöse Glucosebelastung durchgeführt und schließlich das Verhalten der nichtveresterten Fettsäuren nach intravenöser Glucosebelastung zur Diagnose des latenten Diabetes mellitus herangezogen.

Die frühzeitige Diagnose des latenten Diabetes mellitus ist klinisch bedeutungsvoll. Man weiß, daß lange bevor der Diabetes mellitus mit einer Glykosurie manifest wird, ein latenter Diabetes mellitus diabetische Organveränderungen wie Retinopathie, Nephropathie, Polyneuropathie oder eine Arteriosklerose begünstigen kann (10-13). Bei frühzeitiger Erfassung des latenten Diabetes mellitus kann man versuchen, durch eine diätetische Behandlung die Manifestation der diabetischen Komplikationen zu vermeiden oder hinauszuzögern.

\section{Methoden}

1. Intravenöse Glucosebelastungen. - Nach etwa 14 Stunden nächtlichem Fasten wurde bei Patienten morgens zwischen 7.30 Uhr und 9.00 Uhr mit einer intravenösen Glucosebelastung begonnen. Die Patienten hatten das Bett morgens nicht verlassen. Alle Blutentnahmen erfolgten aus einer mäßig gestauten Unterarmvene ohne Aspiration. Nach Abnahme einer Nüchternblutprobe wurde innerhalb von 3-5 Minuten $1 \mathrm{ml}$ einer 50\%igen Glucoselösung pro kg Körpergewicht des Patienten mit einer Rekordspritze injiziert. Sofort danach wurden $20 \mathrm{ml}$ $0,9 \%$ ige $\mathrm{NaCl}$ gespritzt. Während der darauffolgenden Zeit wurde die liegende Kanüle mittels eines 0,9\%igen $\mathrm{NaCl}$-Dauertropfes freigehalten. Von der vierten bis zur 84. Minute nach Beendigung der Glucose-Injektion wurden in etwa zehnminütigen Abständen Blutproben (etwa $8 \mathrm{ml}$ ) in ein Zentrifugenglas abgelassen und sofort in ein Eiswasserbad gestellt. Von dem entnommenen Blut wur-

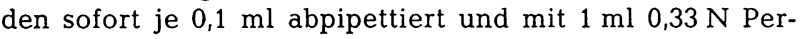
chlorsäure in Spitzgläsern sorgfältig vermischt. - Bei der doppelten intravenösen Glucosebelastung wurde $95 \mathrm{Mi}$ nuten nach Beendigung der ersten Glucose-Injektion am anderen Arm mit einer zweiten Glucose-Injektion $(1 \mathrm{ml}$ einer 50\% \%igen Glucoselösung pro kg Körpergewicht) begonnen. Blutproben von $0,1 \mathrm{ml}$ wurden hierauf von der vierten bis zur 64. Minute nach Beendigung der zweiten Glucose-Injektion in zehnminütigen Abständen für die Glucosebestimmung entnommen.

2. Orale Glucosebelastungen. - Zwei Tage nach der intravenösen Glucosebelastung wurde bei den Patienten unter den entsprechenden Bedingungen eine doppelte orale Glucosebelastung durchgeführt. Die Patienten erhielten nach Abnahme des Nüchternblutzuckers pro kg Körpergewicht $1 \mathrm{ml}$ einer 50\%igen Glucoselösung in $1: 2$ Verdünnung mit Wasser. Dreißig, 60 und 90 Minuten nach dem Trunk wurden Blutproben von $0,1 \mathrm{ml}$ (Kapillarblut) für die Glucosebestimmung abgenommen. Neunzig Minuten nach der ersten oralen Glucosebelastung trank der Patient dieselbe Menge Glucoselösung ein zweites Mal, worauf insgesamt 120,150 und 180 Minuten nach der ersten oralen Glucosebelastung Blutproben entnommen wurden. Die Ergebnisse der oralen Glucosebelastungen wurden nach folgenden Verfahren beurteilt:

a) Die erste orale Glucosebelastung wurde in Anlehnung an die von Williams (14) für die einfache orale Glucosebelastung angegebenen drei Kriterien beurteilt: Nüchternblutzucker unter $100 \mathrm{mg} \%$, Anstieg des Blutzuckers nicht über $160 \mathrm{mg} \%$, Abfall des Blutzuckers nach 90 Minuten auf unter $140 \mathrm{mg} \%$.

b) Beurteilung der doppelten oralen Glucosebelastung nach Staub-Traugott (2). Berücksichtigt wurden vier Kriterien: Nüchternblutzucker unter $100 \mathrm{mg} \%$, erstes Maximum des Blutzuckers nicht höher als der doppelte Nüchternblutzucker, zweites Maximum des Blutzuckers nicht mehr als $20 \mathrm{mg} \%$ höher als das erste Maximum, Endwert des Blutzuckers bei 180 Minuten nicht mehr als $20 \mathrm{mg} \%$ höher als der Nüchternblutzucker. Nach einer Glykosurie während der Glucosebelastung (Kriterium Nr. 5 nach Staub) wurde von uns nicht gesucht.

c) Beurteilung der doppelten oralen Glucosebelastung durch Vergleich mit den Werten eines eigenen Normalkollektivs. Folgende fünf Kriterien wurden berücksichtigt: Nüchternblutzucker unter $95 \mathrm{mg} \%$, erstes Maximum unter $170 \mathrm{mg}^{\%} \%$, erstes Minimum unter $145 \mathrm{mg} \%$, zweites Maximum unter $170 \mathrm{mg} \%$, Endwert unter $140 \mathrm{mg} \%$.

Als pathologisch wurden orale Glucosebelastungen gewertet, bei denen nach Beurteilung a) die Kriterien zwei und drei überschritten waren. Für Beurteilung b) wurde verlangt, daß mindestens zwei der vier Grenzwerte überschritten waren, für Beurteilung c) forderten wir, daß von den Grenzwerten der fünf Kriterien mindestens zwei überschritten wurden.

3. Verhalten der nichtveresterten Fettsäuren nach intravenöser Glucosebelastung. - Blutproben wurden vor und in zehnminütigen Abständen während der oben beschriebenen ersten intravenösen Glucosebelastung zur Bestimmung des Gehaltes an nichtveresterten Fettsäuren entnommen. Die sofort am Krankenbett in ein Eiswasserbad gestellten Blutproben wurden nach Beendigung der Untersuchung 20 Minuten bei etwa $1500 \mathrm{x}$ g zentrifugiert und in dem so gewonnenen Serum die nichtveresterten Fettsäuren nach Dole (15) mit einmaliger Extraktion bestimmt.

4. Glucosebestimmung und Berechnung des sogenannten Glucose-Assimilationskoeffizienten. - Die Glucosebestimmung erfolgte enzymatisch. Die Blutzuckerwerte wurden auf semilogarithmischem Papier gegen die Zeit aufgetragen, und die Halbwertszeit (T) der Glucose wurde, wie früher beschrieben (9), ermittelt. Die Elimination der Glucose wird in etwa durch die Gleichung (1) beschrieben (5) :

$$
\mathrm{C}_{(\mathrm{t})}=\mathrm{C}_{(0)} \cdot \mathrm{e}^{-\mathrm{kt}}
$$

Setzt man statt der variablen Zeit $(t)$ die Halbwertszeit (T) der Glucose, so gilt:

$$
\mathrm{k}=\frac{\ln 2}{\mathrm{~T}}=\frac{0,693}{\mathrm{~T}}
$$

Der k-Wert ist also ein einfacher Quotient, der üblicherweise mit 100 multipliziert wird. Man erhält damit die Glucose-Elimination in \% pro Minute zum Zeitpunkt der Halbwertszeit bzw. den sogenannten Glucose-Assimilationskoeffizienten $\mathrm{k}_{\mathrm{G}}$. Im Gegensatz $\mathrm{zu}$ unserer ersten Mitteilung (9) wurden die ersten Glucosewerte nach der intravenösen Glucosebelastung (4-Minutenwert) nicht berücksichtigt, da nach Franckson und Mitarbeitern (5) erst von der 15. Minute an mit einer ausreichend gleichmäßigen Verteilung der Glucose zu rechnen sein soll. Nach unseren Erfahrungen lag jedoch nur ein Teil der 4-Minuten-Blutzuckerwerte oberhalb der den Blutzucker- 
abfall von der 15. bis zur 65. Minute charakterisierenden Geraden. Aufgrund dieser Modifikation ist der in dieser Arbeit mitgeteilte Normalbereich der $\mathbf{k}_{\mathrm{G}}$-Werte etwas niedriger als der früher beschriebene (9). Blutzuckerwerte unter $80 \mathrm{mg} \%$ wurden für die graphische Ermittlung der Halbwertszeit nicht berücksichtigt. Die einfache intravenöse Glucosebelastung und die Ėrmittlung der Halbwertszeit der Glucose (T) sind in Abbildung 1 schematisch dargestellt.

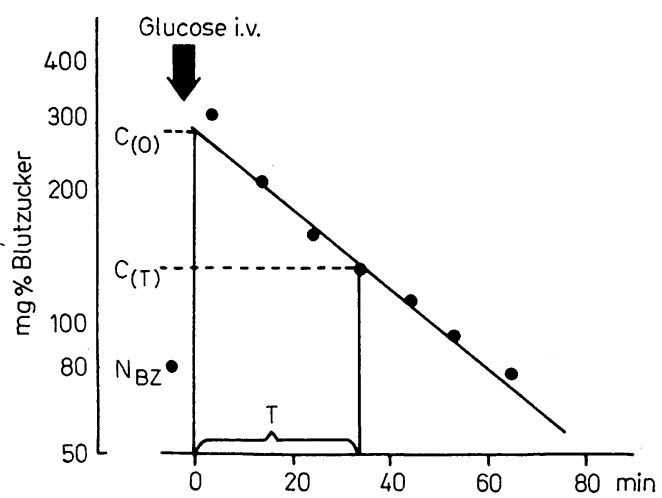

Abb. 1. Schematische Darstellung der graphischen Bestimmung der Halbwertszeit (T) der akut intravenös applizierten Glucose. NBZ: Nüchternblutzucker.

\section{Ergebnisse}

\section{Diagnostische Bedeutung der intravenösen Glucosebelastung}

Am Beispiel eines diabetischen und eines nichtdiabetischen Patienten ist in Abbildung 2 das Verhalten der Blutzuckerwerte bei der doppelten intravenösen Glucosebelastung dargestellt. Bei dem diabetischen Patienten erfolgt der Abfall der Blutzukkerkonzentration nach beiden Glucose-Injektionen langsamer als bei dem stoffwechselgesunden Patienten - die Linie der miteinander verbundenen Blut-

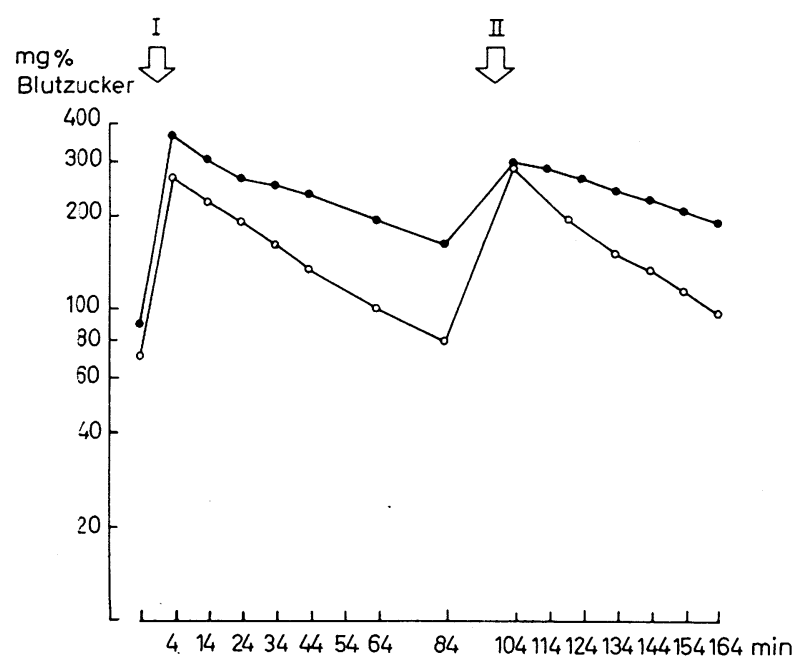

Abb. 2. Doppelte intravenöse Glucosebelastung bei einem nichtdiabetischen und einem diabetischen Patienten. Ein nichtdiabetischer Patient $\left(\mathrm{o}-\mathrm{o}, \mathrm{k}_{\mathrm{G}}=1,51\right)$ wurde doppelt intravenös mit Glucose belastet, in gleicher Weise ein ciabetischer Patient $\left(\bullet-\mathrm{k}_{\mathrm{G}}=0,89\right)$. Dargestellt sind die Zeitpunkte der beiden Belastungen I und II und die Kurven aus den Blutzuckerwerten nach den GlucoseInjektionen. zuckerwerte verläuft flacher. Wie unter Methoden beschrieben, wurde für beide Glucosebelastungen ein Glucose-Assimilationskoeffizient $\left(\mathrm{k}_{\mathrm{G}_{1}}\right.$ und $\mathrm{k}_{\mathrm{G}_{2}}$ ) berechnet.

Die von uns untersuchten Patienten ließen sich nach der Größe des $\mathrm{k}_{\mathrm{G}}$-Wertes der ersten intravenösen Glucosebelastung in fünf Gruppen einteilen: 1. erhöhte $\mathrm{k}_{\mathrm{G}}$-Werte (über 2,2), 2 . normale $\mathrm{k}_{\mathrm{G}}$-Werte $(1,2-2,2)$, 3. Zwischenbereich $\left(\mathrm{k}_{\mathrm{G}}: 1,0-1,2\right)$, 4. erniedrigte $\mathrm{k}_{\mathrm{G}}$-Werte (unter 1,0 ) ohne Glykosurie (= latent diabetisch), 5. erniedrigte $\mathrm{k}_{\mathrm{G}}$-Werte (unter 1,0$)$ mit Glykosurie (= manifest diabetisch).

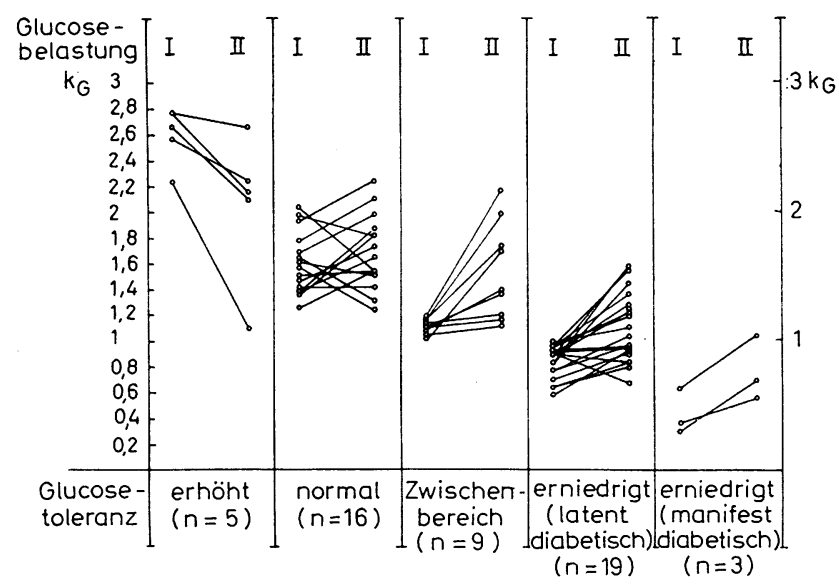

Abb. 3. Verhalten der einzelnen $\mathrm{k}_{\mathrm{G}}$-Werte bei der intravenösen Glucose-Doppelbelastung. Für jede Patientengruppe $1-5$ (Einteilung $\mathrm{s}$. Text) wurden die $\mathrm{k}_{\mathrm{G}}$-Werte der ersten Belastung (I) auf die Ordinate aufgetragen und mit den zugehörigen $\mathrm{k}_{\mathrm{G}}$-Werten der zweiten (II) Belastung (ebenfalls Ordinate) verbunden.

Die für diese Gruppen in Tabelle 1 wiedergegebenen Mittelwerte des Nüchternblutzuckers und der höchsten (Maximum I und II) sowie der niedrigsten unter den beiden Glucosebelastungen erreichten Blutzuckerwerte lassen folgendes erkennen: Die Maxima I und II sind bei den Gruppen der stoffwechselgesunden und der latent-diabetischen Patienten etwa gleich hoch oder zeigen doch eine ganz erhebliche Uberlappung. Ein deutlicher Unterschied zeigt sich dagegen für die niedrigsten nach der ersten und zweiten Glucosebelastung erreichten Blutzuckerwerte (Minimum I und Endwert). Die Kurve der Glucose-Elimination verläuft bei den latent-diabetischen Patienten also deutlich flacher, der $\mathrm{k}_{G}$-Wert ist, wie unter Methoden beschrieben, kleiner. Man erkennt aus diesen Daten, daß der $\mathrm{k}_{\mathrm{G}}$-Wert der ersten intravenösen Glucosebelastung die Geschwindigkeit der GlucoseElimination gut charakterisiert und daß ein Blutzuckerwert von mehr als $107 \mathrm{mg} \%(\overline{\mathrm{x}}+2 \sigma)$ nicht mehr im Normalbereich der Blutzuckerwerte des Minimum I liegt und für eine pathologische Glucosetoleranz spricht. Bei den Patienten mit erhöhtem ersten $\mathrm{k}_{G}$-Wert ist das Minimum nach der ersten intravenösen Glucosebelastung deutlich niedriger als das erste Minimum der Patienten mit normalem ersten $\mathrm{k}_{\mathrm{G}}$-Wert. Deutlich niedriger ist auch das zweite Maximum, wogegen der Endwert der Blutzuckerkurven wieder angenähert in der Höhe des Endwertes der Patienten mit normalem ersten $\mathrm{k}_{\mathrm{G}}$-Wert liegt. Der zweite $\mathrm{k}_{G}$-Wert war daher bei diesen Patienten stets kleiner als der erhöhte erste $\mathrm{k}_{\mathrm{G}}$-Wert (Abbildung 3 und $4)$. 
Tab. 1. Extremwerte der Blutzuckerkurven nach intravenöser und oraler Glucosebelastung. Die Einteilung der Patientengruppen erfolgte wie im Text beschrieben. Für jede Patientengruppe wurde aus allen Extrempunkten der Mittelwert der

Blutzuckerwerte $(\overline{\mathrm{x}} \pm \sigma)$ mit der einfachen Standardabweichung berechnet (19)

Patientengruppe

$$
\mathrm{k}_{\mathrm{G}} \text {-Wert }
$$

Nüchtern-
blutzucker

\begin{tabular}{l|l|l} 
Maximum I & Minimum I & Maximum II
\end{tabular}

Ende

Hauptpunkte bei den intravenösen Glucosebelastungen

\begin{tabular}{|c|c|c|c|c|c|c|}
\hline erhöht & über 2,2 & $\begin{array}{r}78 \\
\pm \quad 6\end{array}$ & $\begin{array}{r}261 \\
\pm 18\end{array}$ & $\begin{array}{r}68 \\
\pm \quad 7\end{array}$ & $\begin{array}{r}229 \\
\pm 51\end{array}$ & $\begin{array}{r}85 \\
+18\end{array}$ \\
\hline normal & $1,2-2,2$ & $\begin{array}{r}81 \\
\pm 10\end{array}$ & $\begin{array}{r}276 \\
\pm 44\end{array}$ & $\begin{array}{r}85 \\
+11\end{array}$ & $\begin{array}{r}279 \\
\pm 53\end{array}$ & $\begin{array}{r}97 \\
\pm 16\end{array}$ \\
\hline Zwischenbereich & $1,0-1,2$ & $\begin{array}{r}87 \\
+10\end{array}$ & $\begin{array}{r}300 \\
\pm 46\end{array}$ & $\begin{array}{r}117 \\
\pm 27\end{array}$ & $\begin{array}{r}327 \\
\pm 57\end{array}$ & $\begin{array}{r}123 \\
\pm 33\end{array}$ \\
\hline latent diabetisch & unter 1,0 & $\begin{array}{r}82 \\
\pm 25\end{array}$ & $\begin{array}{r}275 \\
\pm 105\end{array}$ & $\begin{array}{r}135 \\
\pm 49\end{array}$ & $\begin{array}{r}304 \\
+114\end{array}$ & $\begin{array}{r}150 \\
\pm 62\end{array}$ \\
\hline manifest diabetisch & unter 1,0 & $\begin{array}{r}138 \\
\pm 24\end{array}$ & $\begin{array}{r}326 \\
\pm 21\end{array}$ & $\begin{array}{r}216 \\
\pm 20\end{array}$ & $\begin{array}{r}372 \\
\pm 38\end{array}$ & $\begin{array}{r}231 \\
\pm \quad 23\end{array}$ \\
\hline
\end{tabular}

Hauptpunkte bei der oralen Glucosebelastung

\begin{tabular}{l|l|l} 
erhöht & über 2,2 & \pm \\
normal & $1,2-2,2$ & \pm \\
Zwischenbereich & $1,0-1,2$ & \pm 1 \\
latent diabetisch & unter 1,0 & \pm 1 \\
manifest diabetisch & & \pm 5 \\
& unter 1,0 &
\end{tabular}

Eine Erklärung der sogenannten erhöhten Glucosetoleranz (Abbildung 3) ist nicht ohne weiteres möglich. Es ist bekannt, daß zum Beispiel bei latenter Nebennierenrindeninsuffizienz die Glucosetoleranz erhöht sein kann (16). Körperliche Belastung und Muskelarbeit sollen ebenfalls die Glucosetoleranz erhöhen $(17,51)$. Aus diesem Grunde achteten wir darauf, daß unsere Patienten vor der Untersuchung das Bett nicht verließen.

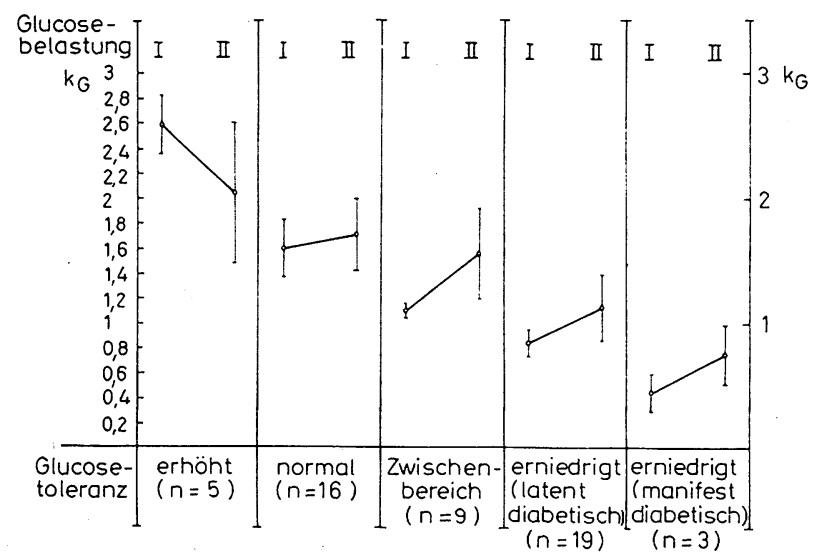

Abb. 4. Verhalten der $\mathrm{k}_{\mathrm{G}}$-Werte bei der intravenösen Glucose-Doppelbelastung (Mittelwert $\pm \sigma$ ). Von den einzelnen $\mathrm{k}_{\mathrm{G}}$-Werten (s. Abb. 3 ) der ersten Belastung wurde der Mittelwert mit der einfachen Standardabweichung $\sigma$ auf der Ordinate für jede der Patientengruppen $1-5$ aufgetragen und mit dem zugehörigen Mittelwert der zweiten Belastung verbunden.
Die sogenannten Glucose-Assimilationskoeffizien-ten der ersten und zweiten intravenösen Glucosebelastung $\left(\mathrm{k}_{\mathrm{i}_{1}}\right.$ und $\mathrm{k}_{\left.\mathrm{i}_{2}\right)}$ ) der von uns untersuchten $\mathrm{Pa}$ tienten sind in Abbildung 3 und 4 miteinander verglichen. Wir fanden eine sehr unterschiedliche Geschwindigkeit der Glucose-Elimination bei der Gruppe der Normalpersonen. Mit Abnahme der Glucosetoleranz zeichnete sich vielleicht eine Tendenz zu höheren $\mathrm{k}_{(\mathrm{i}}$-Werten der zweiten Glucosebelastungen $a b$. Die Bereiche der einfachen Standardabweichungen $(\overline{\mathrm{x}} \pm \sigma)$ der $\mathrm{k}_{\mathrm{G}}$-Werte der zweiten intravenösen Glucosebelastung zeigen dabei eine wesentliche Uberlappung, so daß man schließen darf, daß das Verfahren der doppelten intravenösen Glucosebelastung in der hier mitgeteilten Form keine Verbesserung der Diagnostik des latenten Diabetes mellitus erbringt. Andere Autoren (22) sahen bei dreifacher intravenöser Glucosebelastung bei insulinbedürftigen Diabetikern zuerst einen Anstieg und dann eine Erniedrigung der Eliminationsgeschwindigkeit der Glucose.

Es ist vorgeschlagen worden, in Analogie zur Berechnung des sogenannten Glucose-Assimilationskoeffizienten $\mathrm{k}_{\mathrm{G}}$ einen entsprechenden Koeffizienten für den Glucose-Exzess, das ist der gemessene Glucosewert abzüglich des Nüchternblutzuckers, zu berechnen $(3,6)$. Für zwei Gruppen von jeweils 21 nichtdiabetischen und 21 diabetischen Patienten wurden die Mittelwerte mit der einfachen Standardabweichung der $\mathrm{k}_{\mathrm{G}}$-Werte und der dazugehörigen $\mathrm{k}_{\mathrm{GE}}$-Werte berechnet. Tabelle 2 zeigt, 
daß bei diesen nicht normal verteilten Kollektiven die "Normalbereiche" bei den $\mathrm{k}_{\mathrm{GE}}$-Werten erheblich mehr überlappen als bei den $\mathrm{k}_{(\mathrm{i}}$-Werten (9). Wir geben aus diesem Grunde der Ermittlung des $\mathrm{k}_{\mathrm{G}}$-Wertes den Vorzug.

Voraussetzung für die Angabe eines Normalbereiches für einen biologischen Wert ist bekanntlich eine Normalverteilung der zur Untersuchung gelangenden Bevölkerung. 24 Patienten, die klinisch in keiner Weise auf eine diabetische Stoffwechsellage verdächtig waren, zeigten bei uns tatsächlich eine Normalverteilung ihrer $\mathrm{k}_{(\mathrm{i}}$-Werte. Der von diesem Kollektiv errechnete Normalbereich der $\mathrm{k}_{(\mathrm{i}}$-Werte lag zwischen 1,04 und 2,16 ( $\left.\overline{\mathrm{x}} \pm 2 \sigma\right)$. Wie bereits erwähnt, liegen diese Werte etwas niedriger als die früher von anderen Autoren und auch von uns angegebenen. Der Grund dafür dürfte in erster Linie die strenge Einhaltung der Bettruhe und die ausschließliche Berücksichtigung der Blutzuckerwerte zwischen der 15 . und 65. Minute nach der intravenösen Glucose-Applikation sein. Wie für die $\mathrm{k}_{\mathrm{G}}$-Werte bei der intravenösen Glucosebelastung, so gilt auch für die Angabe von Normalbereichen der Blutzuckerwerte bei oralen Glucosebelastungen, daß man aufgrund der Selektion der zur Untersuchung gelangenden Fälle nicht erwarten kann, daß die in einem größeren klinischen Untersuchungsgut anfallenden, nicht pathologischen Werte normal verteilt sind.

Wie erwähnt, wurden die von uns untersuchten Patienten nach der Höhe des $\mathrm{k}_{\mathrm{G}}$-Wertes der ersten intravenösen Glucosebelastung in fünf Gruppen eingeteilt. Klinische Einzelheiten, wie Alter, Gewichtsangabe in Prozent des Durchschnittsgewichtes, klinische Diagnosen und Einzelwerte der hier mitge-
Tab. 2. Gegenüberstellung der Assimilationskoeffizienten für Glucose $\left(k_{G}\right)$ und Glucose-Exzeß $\left(k_{G E}\right)$ (Mittelwert $\left.\pm \sigma\right)$, einer gesunden und einer diabetischen Patientengruppe. An jeweils 21 Gesunden und 21 Diabetikern wurde der Mittelwert \pm Standardabweichung o (19) der $\mathrm{k}_{\mathrm{G}}$-Werte und der $\mathrm{k}_{\mathrm{GE}}$-Werte bestimmt.

\begin{tabular}{l|c|c}
\hline & $\mathrm{k}_{\mathrm{G}} \pm \sigma$ & $\mathrm{k}_{\mathrm{GE}} \pm \sigma$ \\
\hline Gesunde & 1,60 & 3,30 \\
& $\pm 0,295$ & $\pm 0,815$ \\
Diabetiker & 0,84 & 1,65 \\
& $\pm 0,118$ & $\pm 0,422$ \\
\hline
\end{tabular}

teilten Belastungsproben zur Erfassung eines latenten Diabetes mellitus werden anderweitig beschrieben (18).

Für die Gruppe der Patienten mit erhöhter Glucosetoleranz galt, daß ihr Gewicht $81-97 \%$ des Durchschnittsgewichtes (19) betrug. Nüchternblutzucker und Nüchternwerte nichtveresterter Fettsäuren lagen im Normalbereich Bei 16 Patienten mit einem ersten $\mathrm{k}_{\mathrm{G}}$-Wert zwischen 1,2 und 2,2 lag das Alter zwischen 15 und 52 Jahren und das Gewicht zwischen 74 und $138 \%$ des Durchschnittsgewichtes. Patienten, die wir aufgrund eines $\mathrm{k}_{\mathrm{G}}$-Wertes von 1,0 bis 1,2 dem sogenannten Zwischenbereich zuordneten, wiesen die in Tabelle 3 angegebenen klinischen Daten auf. Patienten mit $\mathrm{k}_{\mathrm{G}^{-}}$-Werten unter 1,0 wurden, sofern sie keine Glykosurie hatten, der Gruppe mit latentem Diabetes zugeordnet. Ihre klinischen Daten sind in Tabelle 4 zusammengefaßt. Drei Patienten mit einem manifesten Diabetes mellitus im Alter von 36, 53 und 63 Jahren ohne wesentliches Ubergewicht wiesen erhöhte

Tab. 3. Werte der doppelten intravenösen und oralen Glucosebelastung und der nichtveresterten Fettsäuren (NFS) bei der Patientengruppe 3. Die Patienten hatten alle bei der ersten intravenösen Glucosebelastung einen $\mathrm{k}_{\mathrm{G}}$-Wert von 1,0 bis 1,2 , der nicht sicher eine Zuordnung zur diabetischen oder nichtdiabetischen Seite zuläßt; dieser Bereich wurde "Zwischenbereich" genannt

\begin{tabular}{|c|c|c|c|c|c|c|c|c|c|}
\hline Patient & $\begin{array}{c}\text { Alter } \\
\text { (Jahre) }\end{array}$ & $\begin{array}{c}0 \% \text { des } \\
\text { Durch- } \\
\text { schnitts- } \\
\text { gewichtes }\end{array}$ & Diagnose & $\begin{array}{l}\text { Nüch- } \\
\text { tern- } \\
\text { blut- } \\
\text { zucker }\end{array}$ & $\begin{array}{r}\text { dopp } \\
\text { venös } \\
\text { bel } \\
\mathrm{k}_{\mathrm{G} 1}\end{array}$ & $\begin{array}{l}\text { ntra- } \\
\text { lcose- } \\
\text { ig }\end{array}$ & $\begin{array}{l}\text { Nü-FFS } \\
(\mu \mathrm{val} / \mathrm{l})\end{array}$ & $\begin{array}{l}\text { Verhalten } \\
\text { der } \\
\text { NFS }\end{array}$ & $\begin{array}{l}\text { doppelte } \\
\text { orale } \\
\text { Glucose- } \\
\text { belastung } \\
\text { Zahl der } \\
\text { patholo- } \\
\text { gischen } \\
\text { Kriterien }\end{array}$ \\
\hline Bü. R. & 29 & $112 \%$ & $\begin{array}{l}\text { Verdacht auf Encephalo- } \\
\text { myelitis disseminata, } \\
\text { Hypertonus }\end{array}$ & $103 \mathrm{mg} / \mathrm{o}$ & 1,15 & 1,20 & 1310 & diabetisch & 4 \\
\hline He. J. & 55 & $100 \%$ & $\begin{array}{l}\text { intermittierendes zere- } \\
\text { brales Ischämiesyndrom, } \\
\text { Hypertonus }\end{array}$ & $85 \mathrm{mg}^{0} / 0$ & 1,05 & 1,12 & 696 & diabetisch & 1 \\
\hline Ko. T. & 53 & $75 \%$ & Zustand nach Billroth II & $84 \mathrm{mg}^{0} / \mathrm{o}$ & 1,19 & 2,16 & 430 & diabetisch & 1 \\
\hline Pa. G. & 20 & $92 \%$ & $\begin{array}{l}\text { Mucoviscidosis } \\
\text { (Erwachsenenform) }\end{array}$ & $76 \mathrm{mg}^{0} / 0$ & 1,02 & 1,69 & 773 & diabetisch & - \\
\hline Hu. J. & 51 & $117 \%$ & $\begin{array}{l}\text { psychogene Gangstörun- } \\
\text { gen }\end{array}$ & $83 \mathrm{mg}^{0} / \mathrm{o}$ & 1,10 & 1,16 & 695 & indifferent & - \\
\hline Be. R. & 24 & $134 \%$ & Cushing-Syndrom & $95 \mathrm{mg} \%$ & 1,17 & 1,98 & 736 & diabetisch & 3 \\
\hline Re.F. & 22 & $175 \%$ & alimentäre Fettsucht & $81 \mathrm{mg} \%$ & 1,17 & 1,73 & 833 & indifferent & - \\
\hline De. A. & 27 & $206 \%$ & Cushing-Syndrom & $56 \mathrm{mg}^{2} / \mathrm{o}$ & 1,14 & - & 1150 & normal & - \\
\hline Ke. F. & 36 & $143 \%$ & alimentäre Fettsucht & $75 \mathrm{mg}^{0} / 0$ & 1,08 & 1,39 & 675 & diabetisch & - \\
\hline Mü. K. & 15 & $96 \%$ & Verdacht auf Sprue & $104 \mathrm{mg}^{0} / 0$ & 1,12 & 1,36 & 535 & normal & 1 \\
\hline
\end{tabular}


Tab. 4. Werte der doppelten intravenösen und oralen Glucosebelastung und der nichtveresterten Fettsäuren (NFS) bei der Patientengruppe 4 (latenter Diabetes). Die Patienten hatten alle in der ersten intravenösen Glucosebelastung einen diabetischen $\mathrm{k}_{\mathrm{G}}$-Wert (unter 1,0), aber keine Glycosurie

\begin{tabular}{|c|c|c|c|c|c|c|c|c|c|}
\hline Patient & $\begin{array}{c}\text { Alter } \\
\text { (Jahre) }\end{array}$ & $\begin{array}{c}0 \% \text { des } \\
\text { Durch- } \\
\text { schnitts- } \\
\text { gewichtes }\end{array}$ & Diagnose & $\begin{array}{l}\text { Nüch- } \\
\text { tern- } \\
\text { blut- } \\
\text { zucker }\end{array}$ & $\begin{array}{r}\text { dopp } \\
\text { venös } \\
\text { bel } \\
\mathrm{k}_{\mathrm{Gi1}}\end{array}$ & $\begin{array}{l}\text { ntra- } \\
\text { lcose- } \\
\text { ig }\end{array}$ & $\begin{array}{l}\text { Nü-FFS } \\
(\mu v a l / l)\end{array}$ & $\begin{array}{l}\text { Verhalten } \\
\text { der } \\
\text { NFS }\end{array}$ & $\begin{array}{l}\text { doppelte } \\
\text { orale } \\
\text { Glucose- } \\
\text { belastung } \\
\text { Zahl der } \\
\text { patholo- } \\
\text { gischen } \\
\text { Kriterien }\end{array}$ \\
\hline Pi. A. & 51 & $125 \%$ & $\begin{array}{l}\text { Zerebralsklerose, Hemi- } \\
\text { Parkinson }\end{array}$ & $92 \mathrm{mg} \%$ & 0,96 & 1,36 & 863 & diabetisch & 2 \\
\hline Ga. G. & 32 & $186 \%$ & Cushing-Syndrom & $82 \mathrm{mg}^{0} / 0$ & 0,92 & 0,96 & 1020 & diabetisch & 1 \\
\hline Ge. F. & 59 & $97 \%$ & $\begin{array}{l}\text { Zerebralsklerose, inter- } \\
\text { mittierendes zerebrales } \\
\text { Ischämiesyndrom }\end{array}$ & $89 \mathrm{mg} \%$ & 0,91 & 1,57 & 722 & indifferent & - \\
\hline Re. J. & 50 & $80 \%$ & $\begin{array}{l}\text { chronische Hepatitis, } \\
\text { Potatorium }\end{array}$ & $99 \mathrm{mg} \%$ & 0,58 & 0,92 & 1122 & diabetisch & - \\
\hline Go. H. & 33 & $86 \%$ & $\begin{array}{l}\text { beginnende Leber- } \\
\text { zirrhose }\end{array}$ & $83 \mathrm{mg}^{\%} / 0$ & 0,89 & 1,24 & 865 & diabetisch & - \\
\hline Mi. F. & 57 & $88 \%$ & $\begin{array}{l}\text { Ulcusnarbe im Bulbus } \\
\text { duodeni, Lungenemphy- } \\
\text { sem }\end{array}$ & $73 \mathrm{mg} \%$ & 0,91 & 0,94 & 378 & normal & - \\
\hline Si. F. & 58 & $109 \%$ & maligner Exophthalmus & $95 \mathrm{mg}^{0} / 0$ & 0,96 & 1,54 & 562 & diabetisch & 4 \\
\hline Ho. R. & 59 & $90 \%$ & $\begin{array}{l}\text { Hustensynkopen, Pneu- } \\
\text { bronchitis }\end{array}$ & $86 \mathrm{mg}^{0} / 0$ & 0,83 & 1,57 & 683 & diabetisch & 1 \\
\hline To. A. & 32 & $90 \%$ & $\begin{array}{l}\text { Colitis ulcerosa, Colica } \\
\text { mucosa }\end{array}$ & $80 \mathrm{mg}^{0} \%$ & 0,99 & 1,10 & 731 & diabetisch & 3 \\
\hline Bo. P. & 62 & $93 \%$ & Bronchialkarzinom & $78 \mathrm{mg}^{0} \%$ & 0,91 & 1,28 & 646 & indifferent & $\begin{array}{l}\text { nicht aus } \\
\text { geführt }\end{array}$ \\
\hline Ba. L. & 23 & $113 \%$ & $\begin{array}{l}\text { Cushing-Rezidiv nach } \\
\text { subtotaler Adrenal- } \\
\text { ektomie }\end{array}$ & $79 \mathrm{mg} \%$ & 0,77 & - & 767 & diabetisch & 3 \\
\hline Schm. K. & 30 & $110 \%$ & chronische Pyelonephritis & $90 \mathrm{mg}^{0} / 0$ & 0,89 & 0,83 & 990 & diabetisch & 5 \\
\hline Ge. P. & 20 & $123 \%$ & $\begin{array}{l}\text { Pyelonephritis, Hyper- } \\
\text { tonus }\end{array}$ & $79 \mathrm{mg} \%$ & 0,95 & 1,21 & 1700 & diabetisch & - \\
\hline Hö. A. & 40 & $143 \%$ & Hypothyreose & $72 \mathrm{mg}^{0} / 0$ & 0,64 & 0,81 & 796 & diabetisch & 1 \\
\hline Kn. F. & 53 & $88 \%$ & $\begin{array}{l}\text { Leberzirrhose, Hämo- } \\
\text { chromatose }\end{array}$ & $91 \mathrm{mg} \%$ & 0,96 & 1,19 & 798 & diabetisch & 1 \\
\hline St. F. & 61 & $113 \%$ & Myasthenia & $98 \mathrm{mg}^{0} / 0$ & 0,70 & 0,91 & 1210 & diabetisch & 4 \\
\hline Schu. J. & 25 & $158 \%$ & alimentäre Fettsucht & $97 \mathrm{mg}^{0} / 0$ & 0,77 & 0,96 & 880 & diabetisch & 1 \\
\hline Ca. M. & 28 & $94 \%$ & $\begin{array}{l}\text { Stenose an der Papilla } \\
\text { Vateri }\end{array}$ & $77 \mathrm{mg} \%$ & 0,90 & 0,67 & 631 & diabetisch & - \\
\hline Ho. F. & 41 & $111 \%$ & Endangitis obliterans & $98 \mathrm{mg} \%$ & 0,83 & 1,44 & 610 & diabetisch & 4 \\
\hline Mi. A. & 45 & $94 \%$ & $\begin{array}{l}\text { Herpes zoster, } \\
\text { Subarachnoidalblutung }\end{array}$ & $82 \mathrm{mg} \%$ & 0,77 & 1,03 & 404 & indifferent & 3 \\
\hline
\end{tabular}

Nüchternblutzucker-, $\mathrm{k}_{(\mathrm{i}}$-Werte, die weit unter 1,0 lagen, sowie ein pathologisches Verhalten der nichtveresterten Fettsäuren und bei der doppelten oralen Glucosebelastung auf.

\section{Diagnostische Bedeutung der oralen Glucosebelastung}

Die oralen Glucosebelastungen wurden, wie unter Methoden beschrieben, durchgeführt. Beurteilt man die fünf Patientengruppen nach dem unter $\mathrm{Me}$ thoden mit a) bezeichneten Verfahren so wie eine einfache orale Glucosebelastung, so zeigten von 19 nach dem Ergebnis der einfachen intravenösen Glucosebelastung als latent diabetisch zu bezeichnenden Patienten (Tabelle 4) 15 in einem Kriterium, acht in zwei und drei in allen drei Kriterien ein pathologisches Verhalten. Vier Patienten wiesen in keinem der drei Kriterien ein Abweichen von dem normalen Verhalten auf. Beurteilt man die oralen Glucosebelastungen nach der Methode b), so zeigten von den 19 Patienten 17 in einem, zehn in zwei und zwei in drei Kriterien ein pathologisches Verhalten. Zwei 
Patienten wiesen in allen vier Kriterien ein normales V'erhalten auf. Nach der Beurteilung c) zeigten von den 19 Patienten 13 in einem, acht in zwei, vier in vier und zwei in allen fünf Kriterien ein pathologisches Verhalten. Sechs Patienten zeigten ein vollständig normales Verhalten. Der Ausfall der oralen Gluc osebelastung war nach den unter Methoden angeführten Prinzipien nach Beurteilung a) bei sieben, nach Beurteilung b) bei zehn und nach Beurteilung $\mathrm{c}$ ) bei acht von 19 latent diabetischen Patienten als pathologisch zu bezeichnen. Bei sieben der $19 \mathrm{~Pa}-$ tienten lag das zweite Maximum der oralen Glucosedoppelbelastung höher als das erste Maximum. Für die Beurteilung c), also nach eigenen statistischen Daten, ist noch zu berücksichtigen, daß als obere Grenze der Maxima I und II nur der ungefähre Bereich der einfachen Standardabweichung gewählt wurde. Setzte man statt dieser Grenzwerte den Normalbereich ein, so würde sich die Trefferquote der oralen Glucosedoppelbelastung noch verschlechtern.

Die Patienten des "Zwischenbereiches" (Tabelle 3) waren aufgrund der oralen Glucosebelastung fast alle als nichtdiabetisch zu bezeichnen. Von den Patienten mit normalem $\mathrm{k}_{\mathrm{G}}$-Wert wies ein Patient, der an einer myatrophischen Lateralsklerose litt, eine als pathologisch zu bezeichnende orale Glucosetoleranz auf, bei diesem Leiden ein häufiger Befund (20). Mittelwerte und Standardabweichung der Blutzuckerwerte bei der oralen Glucosebelastung sind in der unteren Hälfte der Tabelle 1 für die Patientengruppen $1-5$ wiedergegeben.

\section{Diagnostische Bedeutung des Verhaltens der nicht-} veresterten Fettsäuren bei intravenöser Glucosebelastung

Wie unter Methoden beschrieben, wurde das Verhalten der Konzentration an nichtveresterten Fettsäuren im Serum unter der ersten intravenösen Glucosebelastung untersucht. Abbildung 5 zeigt das Verhalten der Blutzuckerkonzentrationen zusammen mit den Veränderungen des Gehalts an nichtveresterten Fettsäuren während 84 Minuten nach der ersten Glucose-Injektion bei einem diabetischen und einem nichtdiabetischen Patienten. Nach einem initialen Anstieg der nichtveresterten Fettsäuren, der möglicherweise durch eine Adrenalininkretion infolge der Manipulationen während der Glucose-Injektion (21) bedingt ist, erfolgte bei dem diabetischen Patienten ein kontinuierlicher Abfall der nichtveresterten Fettsäuren bis zur 84. Minute, während bei dem nichtdiabetischen Patienten die Werte nach der 44. Minute wieder ansteigen. Es war Ziel der hier mitgeteilten Untersuchungen, festzustellen, ob dieser Unterschied im Verhalten der nichtveresterten Fettsäuren zur Diagnose des latenten Diabetes mellitus verwertet werden kann.

Um diese Frage zu klären, wurden die von uns untersuchten Patienten in drei Gruppen eingeteilt: nichtdiabetische Patienten mit einem $\mathrm{k}_{\mathrm{G}}$-Wert über 1,2 ( $\mathrm{n}=24)$, diabetische Patienten mit einem $\mathrm{k}_{\mathrm{G}^{-}}$-

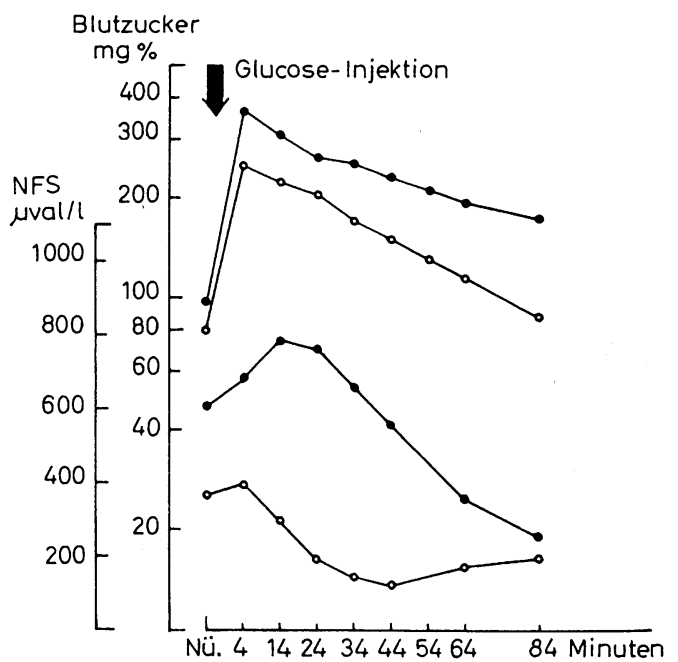

Abb. 5. Verhalten der Blutzuckerkonzentration und des Spiegels der nichtveresterten Fettsäuren im Serum eines diabetischen und eines nichtdiabetischen Patienten nach einer intravenösen Glucosebelastung. Die oberen zwei Kurven geben die Blutzuckerkonzentrationen in $\mathrm{mg}^{\%} \%$ wieder, die unteren beiden die Konzentrationen der nichtveresterten Fettsäuren (NFS) in $\mu \mathrm{val} / \mathrm{l}$. (o-o, $\mathrm{k}_{\mathrm{G}}=1,39$, nichtdiabetisch; $\bullet-\mathrm{k}_{\mathrm{G}}=0,83$, latent diabetisch.)

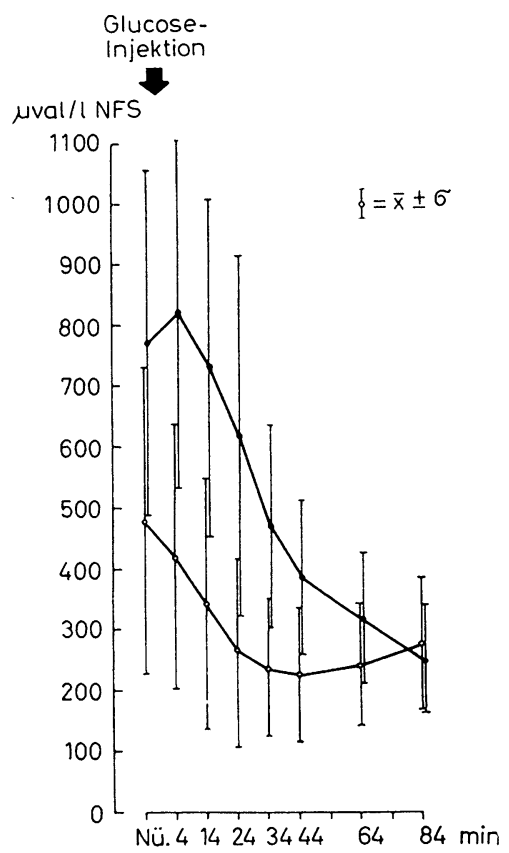

Abb. 6. Verhalten des Spiegels der nichtveresterten Fettsäuren (NFS) im Serum von diabetischen und nichtdiabetischen Patienten. Die obere Kurve zeigt die Mittelwerte mit der einfachen Standardabweichung von den

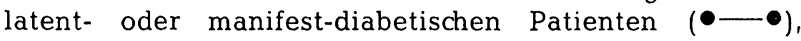
darunter die Kurve aus den Mittelwerten mit der einfachen Standardabweichung von den nichtdiabetischen Patienten $(\mathrm{O}-\mathrm{O})$.

Wert von unter 1,0 $(\mathrm{n}=23)$ und schließlich Patienten, die dem sogenannten Zwischenbereich zugeordnet wurden mit $\mathrm{k}_{\mathrm{G}}$-Werten zwischen 1,0 und 1,2 $(\mathrm{n}=10)$. Abbildung 6 zeigt die Mittelwerte mit der einfachen Standardabweichung der Konzentrationen an nichtveresterten Fettsäuren im Serum von dia- 


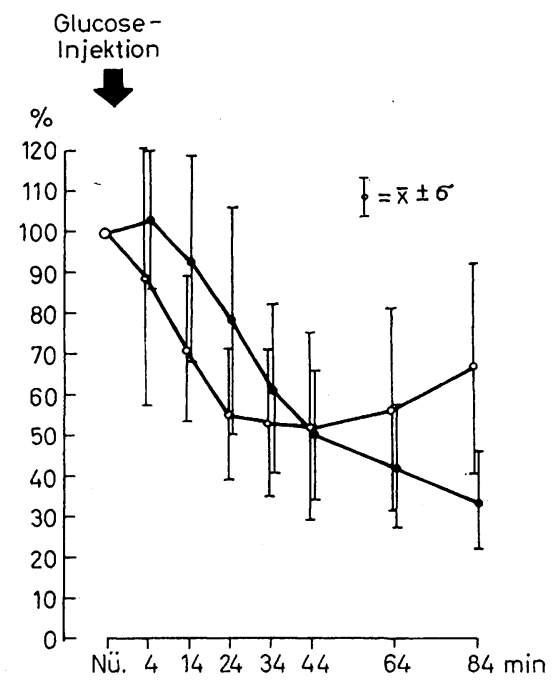

Abb. 7. Verhalten des Spiegels der nichtveresterten Fettsäuren im Serum von diabetischen und nichtdiabetischen Patienten, ausgedrückt in Prozent des Ausgangswertes. Der Nüchternwert der nichtveresterten Fettsäuren aller Patienten wurde gleich $100 \%$ gesetzt. Die Werte der nichtveresterten Fettsäuren nach einer Glucose-Injektion wurden dann in Prozent des Nüchternwertes angegeben. Von beiden Patientengruppen $(-$ latent oder manifest diabetisch; o- o nichtdiabetisch) wurden die Mittelwerte mit der einfachen Standardabweichung für die auf der Abszisse angegebenen Zeitpunkte errechnet und miteinander verbunden.

betischen und nichtdiabetischen Patienten. In Abbildung 7 wurde der unterschiedlich hohe Nüchternwert der nichtveresterten Fettsäuren dadurch ausgeglichen, daß dieser gleich $100 \%$ gesetzt wurde und die Werte der nichtveresterten Fettsäuren nach. der Glucose-Injektion in Prozent des Ausgangswertes angegeben wurden. Bei den diabetischen Patienten folgt nach einem kurzen Anstieg der nichtveresterten Fettsäuren innerhalb der ersten vier Minuten nach Glucose-Injektion ein kontinuierlicher $\mathrm{Ab}$ fall bis zur 84. Minute der Untersuchung. Bei den nichtdiabetischen Patienten steigt der 4-MinutenWert der nichtveresterten Fettsäuren gegenüber dem Nüchternwert im Mittel dagegen nicht an; die Konzentration im Serum fällt etwa bis zur 44. Minute, darauf folgt ein Wiederanstieg der nichtveresterten Fettsäuren bis zur 84. Minute, so daß der Endwert höher liegt als bei den diabetischen Patienten.

Auch nach unseren Untersuchungen war es nicht möglich, Diabetiker und Nichtdiabetiker aufgrund der verschiedenen Höhe der Nüchternkonzentration an nichtveresterten Fettsäuren im Serum klar voneinander zu trennen; auch die Berücksichtigung der unterschiedlichen Geschwindigkeit (Untersuchungen mit H. Klenk), mit der die nichtveresterten Fettsäuren aus dem Serum eliminiert werden, ergab keine signifikanten Unterschiede zwischen den beiden Gruppen. Wir haben daher das Verhalten der nichtveresterten Fettsäuren bei den einzelnen Patienten nach folgenden drei Kriterien beurteilt: normal: Wiederanstieg der nichtveresterten Fettsäuren, spätestens nach der 64. Minute, indifferent: Abfall bis zur 64. Minute und Gleichbleiben der nichtveresterten Fettsäuren zwischen der 64. und 84. Minute, diabetisch: kontinuierlicher Abfall der nichtveresterten Fettsäuren über die 64. Minute hinaus.

Vergleicht man das Verhalten der nichtveresterten Fettsäuren nach intravenöser Glucosebehandlung mit den zum gleichen Zeitpunkt ermittelten $\mathrm{k}_{\mathrm{G}}$-Werten, die der Einteilung der Patienten in Gruppen zugrunde gelegt wurden, so ergibt sich, daß bei 21 Patienten mit $\mathrm{k}_{\mathrm{G}}$-Werten über 1,2 dreimal der indifferente und 18mal der normale Verhaltenstyp der nichtveresterten Fettsäuren beobachtet wurde. Bei zwanzig latent-diabetischen Patienten (Tabelle 4) und drei manifest-diabetischen Patienten fand sich $19 \mathrm{mal}$ der diabetische, dreimal der indifferente und einmal ein normaler Typ des Verhaltens der nichtveresterten Fettsäuren. Von zehn Patienten, die nach ihrem $\mathrm{k}_{(\mathbf{i}}$-Wert zwischen 1,0 und 1,2 bei der ersten intravenösen Glucosebelastung dem "Zwischenbereich" zugeordnet wurden, wiesen je zwei Patienten ein normales bzw. ein indifferentes die übrigen ein diabetisches Verhalten der nichtveresterten Fettsäuren auf. Die Korrelation zwischen der Höhe des sogenannten Glucose-Assimilationskoeffizienten $\mathrm{k}_{\mathbf{6}}$, und dem Verhalten der nichtveresterten Fettsäuren nach intravenöser Glucosebelastung wird von uns als Hinweis für die Berechtigung der Einteilung der Patienten nach der Höhe des $\mathrm{k}_{(\mathrm{i}}$-Wertes in die oben beschriebenen Gruppen angesehen.

\section{Diskussion}

Zur Erkennung eines Diabetes mellitus ist die einfache Bestimmung des Nüchternblutzuckers nicht geeignet, da dieser Wert von vielen Faktoren verändert werden kann und meist erst im Stadium des manifesten Diabetes regelmäßig erhöht ist. Als empfindlichere Methoden sind in der Klinik seit Jahren die oralen Glucosebelastungen eingeführt, wobei die Glucose als einfache Gabe oder als doppelte nach Staub-Traugott verabreicht wird (2).

Das Verhalten der Blutzuckerwerte nach einer oralen Glucosebelastung ist von zahlreichen sehr variablen Faktoren, unter anderem auch von der individuellen $\mathrm{Re}$ sorptionsgeschwindigkeit $(24,50)$ abhängig und zeigt einen entsprechend großen Streubereich. Außerdem wird die endogene Insulininkretion nach oraler Glucosebelastung stärker stimuliert als nach intravenöser Gabe, was von Elrick und Mitarbeitern (25) durch die höhere Blutzuckerkonzentration in der Pfortader nach oraler Zufuhr erklärt wurde. Neuere Untersuchungen sprechen allerdings dafür, daß ein jejunaler Faktor, vielleicht Sekretin, für die höhere Insulinausschüttung nach oraler Glucosegabe eine Rolle spielt $(26,27)$.

Vergleicht man die Beurteilung der ermittelten Glucosetoleranzen nach oraler und intravenöser 
Glucosebelastung, dann findet man in der Literatur oft sehr verschiedene Angaben. Wie aus den Ergebnissen unserer Arbeit über vergleichende Untersuchungen hervorgeht, zeigten von 19 Patienten, die nach dem intravenösen Glucosetoleranztest einen latenten Diabetes hatten, nur sieben bis zehn Patienten bei der oralen Glucosebelastung pathologische Blutzuckerwerte. Nach unseren Erfahrungen kann somit die orale Glucosebelastung in der von uns angewandten Form nicht als eine genügend empfindliche und brauchbare Methode zur Abklärung eines latenten Diabetes mellitus bezeichnet werden; sie ist jedenfalls dem intravenösen Glucosetoleranztest unterlegen.

Zahlreiche Autoren halten immer noch die sehr verbreitete orale Glucosebelastung und die dabei errechnete Glucosetoleranz für vorteilhafter. Unterschiede des methodischen Verfahrens bedingen dabei zum Teil die divergierenden Befunde. So ist es unseres Erachtens von Bedeutung, die oral verabreichte Glucosemenge auf das Körpergewicht $\mathrm{zu}$ beziehen und nicht einfach $100 \mathrm{~g}$ Glucose zu geben. Außerdem halten viele Angaben einer kritischen Vergleichsanalyse nicht stand, wenn zum Beispiel die Patienten vor der Glucosebelastung nicht einwandfreie Grundumsatzbedingungen einhalten, das heißt wenn die Glucosebelastung bei ambulanten Patienten durchgeführt wird. Wir haben bei unseren stationären Patienten besonderen Wert auf absolute Bettruhe vor der Bestimmung der Glucosetoleranz gelegt, während andere Autoren bei zum Teil ambulanten Patienten höhere Normalbereiche der $\mathrm{k}_{\mathrm{i}}$-Werte fanden. Weiterhin sollte bei der Auswertung der Normalbereiche von kWerten das Körpergewicht berücksichtigt werden, weil übergewichtige Personen häufig zu tieferen (31) und untergewichtige Personen zu höheren k-Werten (29) tendieren. Es ist deshalb bei Vergleichsuntersuchungen über die Wertigkeit der oralen bzw. intravenösen Glucosebelastung für die Beurteilung wichtig, die jeweiligen methodischen Abweichungen und die verschiedenen gebräuchlichen Definitionen der Schweregrade der Kohlenhydratstoff wechselstörung zu berücksichtigen.

Taton und Mitarbeiter (28) kommen in ihrer Studie über Glucosetoleranzstörungen bei genetisch belasteten Patienten $\mathrm{zu}$ von unseren Befunden abweichenden Ergebnissen und halten die orale Glucosebelastung (100 g) für die empfindlichere Methode. Bei Verwandten von Diabetikern, Kindern diabetischer Eltern, eineiigen Zwillingsgeschwistern von Diabetikern und auch bei Kindern eines diabetischen Elternteiles, welche größtenteils keinen latenten oder "chemischen" Diabetes mellitus hatten, war die Glucosetoleranz gegenüber einer Kontrollgruppe ohne Diabetes in der Verwandtschaft im Mittel niedriger. Bei diesen Untersuchungen fanden sich bei oraler Glucosebelastung mehr verdächtige Werte als bei intravenöser Glucosebelastung. Wir gaben einmal nur $0,5 \mathrm{~g}$ Glucose pro kg Körpergewicht und konnten ferner die einfache orale Glucosebelastung in unserer Studie nur bis zur 90. Minute auswerten, weil zu diesem Zeitpunkt die zweite orale Glucosebelastung erfolgte. Bei der einfachen oralen Glucosebelastung gilt gerade der 2- und 3-Stundenwert des Blutzuckers als besonders aussagekräftig (29).

Die einfache orale Glucosebelastung und ihre Reproduzierbarkeit ist kürzlich kritisch von McDonald und Mitarbeitern (52) untersucht worden. In vergleichenden Untersuchungen fanden Nadon und Mitarbeiter (30) mit dem oralen Glucosetoleranztest (100 g pro Person) keine größere Treffsicherheit bei der Diagnose eines latenten Diabetes mellitus als mit dem intravenösen Glucosetoleranztest. Die Ergebnisse beider Verfahren stimmten allerdings häufig nicht überein. Als Nachteile zitieren diese Autoren die zeitaufwendige Technik und Auswertung sowie das Auftreten von Thrombophlebitiden (6 von 83 Patienten) durch die Injektion der hypertonen Glucoselösung. Ein Grund der geringen Treffsicherheit des intravenösen Glucosetoleranztests liegt wohl darin daß die Autoren $\mathbf{k}$-Werte als sogenannte Koeffizienten für den Glucose-Exzess $\left(\mathrm{k}_{\mathrm{GE}}\right)$ berechneten, die nach unseren Untersuchungen zur Diagnostik weniger geeignet sind, weil die $\mathrm{k}_{\mathrm{GE}}$-Werte der untersuchten Kollektive weit mehr als die $k_{G}$-Werte überlappen. Nach unseren Befunden liegt außerdem der angegebene Grenzwert $\mathrm{k}_{\mathrm{GE}}=1,1$ für die Unterscheidung der Diabetiker und Nichtdiabetiker zu niedrig.

Für Vorteile des intravenösen Glucosetoleranztests halten wir in Ubereinstimmung mit Lundbaek (29) den geringeren Zeitaufwand von 90 Minuten gegenüber drei Stunden, die Umgehung möglicher Störfaktoren durch den Magen-Darm-Trakt und die einfache Ermittlung des $\mathbf{k}_{(i}$-Wertes (9). Wir haben einige 100 intravenöse Glucosebelastungen routinemäßig in der Klinik durchgeführt und sahen nicht häufiger Thrombophlebitiden als nach einer einfachen Venenpunktion bei Blutentnahme, allerdings wird bei uns nach der Glucose eine isotone $\mathrm{NaCl}$ Infusion gegeben. - Es ist eine Frage der Definition, in welche Stadien die Frühphasen des Diabetes mellitus eingeteilt werden. In Anlehnung an die Definition von Fajans, Levine und anderen $(1,32,33,49)$ halten wir uns an die allgemein anerkannte Einteilung, wie einleitend beschrieben.

Es ist unseres Erachtens heute nicht gerechtfertigt, abweichende Definitionen einzuführen, wie etwa den sogenannten Prädiabetes mit bereits herabgesetzter Glucosetoleranz, die durch erniedrigte $\mathrm{k}$-Werte belegt wird $(34,35)$. Diese Gruppe entspricht in unseren Untersuchungen dem Stadium des latenten Diabetes mellitus. Die Bestimmung der Glucosetoleranz kann wenig zur Definition des Diabetes mellitus beitragen, sie kann aber nach Lundbaek (29) den Schweregrad der diabetischen Glucosetoleranzstörung definieren. Obwohl wir viele Faktoren kennen, die in der Pathogenese des Diabetes von Bedeutung sind, ist eine klare Definition des Diabetes mellitus bis heute nicht möglich. Nach dem Stand der modernen Diabetesforschung ist es aber berechtigt, diese ätiologisch und pathogenetisch vielfältige Krankheit entsprechend ihrer Entwicklung in verschiedene, klar definierbare Stadien zu unterteilen, nämlich in den Prädiabetes, latenten Diabetes und manifesten Diabetes mellitus. Für die Diagnostik ist der intravenöse Glucosetoleranztest nur für die Phase des latenten und leichten manifesten Diabetes sehr wertvoll bzw. zum relativ sicheren Ausschluß einer solchen Erkrankung gut geeignet.

Größere Untersuchungsreihen eines nicht vorher selektierten Krankengutes ergeben beim intravenösen Glucosetoleranztest stets einen fließenden Ubergang von $\mathrm{k}_{\mathrm{G}}$-Werten, vom diabetischen bis zum normalen Bereich. Unser Normalbereich für $\mathrm{k}_{\mathrm{G}}$-Werte aus einem eigenen statistisch normal verteilten Kollektiv, bei dem klinisch eine diabetische Stoffwech- 
sellage nicht zu vermuten war, lag zwischen 1,04 und 2,16 (Mittelwert $=1,60$ mit zweifacher Standardabweichung). Dieser Wert entspricht den von Lundbaek (29) mitgeteilten von 1,72 bzw. bei Patienten über 50 Jahre von 1,60 und stimmt ebenfalls mit den von Conard (36) errechneten Werten überein. Die Grenze der $\mathbf{k}_{\mathrm{G}}$-Werte $\mathrm{zwischen}$ dem diabetischen und dem normalen Bereich kann nicht scharf gezogen werden, weil dazwischen ein unklarer Ubergang liegt, in welchem die $\mathrm{k}_{\mathrm{G}}$-Werte nicht eindeutig der diabetischen oder nichtdiabetischen Seite zugeordnet werden können. In diesem "Zwischenbereich" der $\mathrm{k}_{\mathrm{G}}$-Werte zwischen 1,0 und 1,2 - bei Lundbaek zwischen den $\mathrm{k}_{\mathrm{G}}$-Werten von 0,9 und 1,05 - ist eine Aussage über eine diabetische Stoffwechselstörung unsicher, weil sich beide Gruppen überlappen. Auch die doppelte intravenöse Glucosebelastung führt hier, wie im Ergebnisteil berichtet, zu keiner besseren Differenzierung. Uber die Provokationsmethode des sogenannten Prednisolon-GlucoseToleranztests nach Fajans (37) zur Klärung fraglicher Glucosetoleranzen im Zwischenbereich wird von manchen Autoren (38) Vorteilhaftes berichtet.

Gerade in jenen unklaren Fällen mit $\mathbf{k}_{\mathrm{G}}$-Werten im Zwischenbereich genügt das alleinige Kriterium der Glucosetoleranz nicht, um die Diagnose eines latenten Diabetes mellitus zu stellen. In solchen Fällen halten wir es für notwendig, ein zweites Kriterium heranzuziehen, um eine diabetische Stoffwechselstörung zu beweisen. Wegen der bekannten engen Beziehungen und Wechselwirkungen zwischen dem Kohlenhydrat- und Fettstoffwechsel erschien es uns sinnvoll, das Verhalten der nichtveresterten Fettsäuren während der intravenösen Glucosebelastung zu verwerten.

Beim manifesten Diabetes mellitus sind die nichtveresterten Fettsäuren im Nüchternserum erhöht $(31,39-43)$, ebenso bei der Adipositas, bei Hyperthyreosen und bei Leberzirrhosen (44-47), wobei der Diabetes mellitus ein häufiges Symptom dieser Krankheitsbilder ist. Erhöhte Serumkonzentrationen der nichtveresterten Fettsäuren hemmen die Insulinwirkung auf die Muskulatur bzw. den Glucosestoffwechsel, so daß Randle (48) den "GlucoseFettsäure-Zyklus" als wichtigen pathogenetischen Mechanismus beim Diabetes mellitus postuliert. In den eigenen Untersuchungen waren die Mittelwerte der nichtveresterten Fettsäuren bei Diabetikern mit erniedrigten $\mathrm{k}_{\mathrm{G}^{-}}$ Werten mit $769 \mu \mathrm{val}$ deutlich höher als bei Gesunden mit $478 \mu \mathrm{val}$. Nach intravenöser Glucosebelastung erfolgt der charakteristische Abfall der nichtveresterten Fettsäuren in beiden Gruppen bis zur 44. Minute. Im Gegensatz zu Gesunden, bei denen die nichtveresterten Fettsäuren spätestens nach der 64. Minute wieder ansteigen, erfolgt bei Diabetikern kein Wiederanstieg, sondern ein kontinuierlicher Abfall über die 64. bis zur 84. Minute. Dieses Ausbleiben des Wiederanstieges der nichtveresterten Fettsäuren nach Glucose-Injektion haben wir als Zeichen einer diabetischen Stoffwechselstörung gewertet und konnten eine gute Ubereinstimmung mit den gleichzeitig erniedrigten bzw. suspekten $k_{G}$-Werten feststellen.

Shafrir (49) hat das Verhalten der nichtveresterten Fettsäuren nach oraler Glucosebelastung untersucht und

für Gesunde einen Abfall bis unter $200 \mu$ val gefordert, obwohl $40 \%$ seiner Patienten mit einem leichten Diabetes ein solches "Normalverhalten" zeigten. Auch wir konnten eine Einteilung von Diabetikern in verschiedene Gruppen nach der Steilheit des Abfalles der nichtveresterten Fettsäuren nach einer intravenösen Glucosebelastung nicht treffen. Dementsprechend können wir dem Vorschlag von Shafrir nicht zustimmen, einen sogenannten $\mathrm{k}_{\mathrm{F}}$-Wert zur Erfassung einer diabetischen Stoffwechselstörung zu errechnen. Die Steilheit des Abfalles der nichtveresterten Fettsäuren ist nämlich abhängig von der jeweiligen Höhe der Nüchternwerte, welche sehr schwanken und zwischen Gesunden und Diabetikern beachtlich überlappen.

\section{Zusammenfassung}

Beim Vergleich der verschiedenen Belastungsmethoden zur Diagnose eines latenten Diabetes mellitus halten wir nach unseren Erfahrungen den einfachen intravenösen Glucosetoleranztest mit der Bestimmung des sogenannten Glucose-Assimilationskoeffizienten $\mathrm{k}_{\mathrm{G}}$ für die einfachste und sicherste Untersuchungsmethode. Sie ist der doppelten oralen Glucosebelastung nach Staub-Traugott überlegen. Eine doppelte intravenöse Glucosebelastung mit Bestimmung der doppelten $\mathrm{K}_{\mathrm{G}_{i}}$-Werte bringt keine Verbesserung der Diagnostik. Eine mit der einfachen intravenösen Glucosebelastung koordinierte Bestimmung des Verhaltens der nichtveresterten Fettsäuren nach der intravenösen Glucosebelastung erhöht die Treffsicherheit in der Diagnostik; die Kombination kann vor allem eine Klärung in jenen Fällen aus dem unklaren Zwischenbereich sicher diabetischer und sicher normaler $\mathrm{k}_{\mathrm{G}}$-Werte bringen und beweist, daß "diabetische" $\mathrm{k}_{\mathrm{G}}$-Werte ohne Glykosurie tatsächlich einem latenten Diabetes mellitus entsprechen.

\section{Literatur}

(1) Camerini-Dávalos, R. A. J. B. Caulfield, S. B. Rees, O. Lozano-Castaneda, S. Naldjian, A. Marble: Diabetes 12 (1963), 508.

(2) Staub, H.: Klin. Wschr. 43 (1965), 61.

(3) Amazuto, D. S., F. L. Stutzman, M. S. Vanderbilt, S. Nesbitt: J. clin. Invest. 32 (1963), 428.

(4) Conard, V., I. R. M. Franckson, P. A. Bastenie, J. Kestens, L. Kovacs: Arch. int. Pharmacodyn. 93 (1953), 277.

(5) Franckson, J. R. M., H. A. Ooms, R. Bellans, V. Conard, P. A. Bastenie: Metabolism 11 (1962), 482

(6) Hlad, C. J., H. Elrick, T A. Witten, A. Smith, Y. Arai, T. Bow: J. clin. Invest. 35 (1956), 1139.

(7) Hamilton, B., A. F. Stein: J. Lab. clin. Med. 27 (1942) 491.

(8) Jörgensen, S., T. Plum:
Acta med. scand. 58 (1923), 161.

(9) Scriba, P. C., K. Schwarz: Münch. med. Wschr. 106 (1964), 1522.

(10) Fabrykant, M., M. L. Gelfand: Amer. J. med. Sci. 247 (1964), 665

(11) Büchele, S.: Schweiz. med. Wschr. 92 (1962), 742.

(12) Ellenberg, M.: J. Amer med. Ass. 183 (1963), 926.

(13) Böhle, E., W. Schrade Münch. med. Wschr. 102 (1960), 565.

(14) Williams, R. H.: Textbook of Endocrinology, 3 . Aufl. (Philadelphia-London 1962), S. 617

(15) Dole, V. P., H. Meinertz: J. biol. Chem. 235 (1960) 2595.

(16) Schwarz, K., P. C. Scriba, G. G. Hofmann: Verh. dtsch. Ges. inn. Med. 71 (1965), 360 . (17) Issekutz, B., H. Miller: Proc. Soc. exp. Biol. (N.Y.) 110 (1962), 237.

(18) Hofmann, G. G.: Diss. München 1966 
(19) Diem, K.: Documenta Geigy, Wissenschaftl. Tabellen. (Basel 1960)

(20) Steinke, J., H. R. Tyler: Metabolism 13 (1964), 1376. (21) Bogdonoff, M. D., E. H. Estes, D. Trout: Proc. Soc. exp. Biol. (N.Y.) 100 (1959), 503.

(22) Streeten, D. H. P., M. M Gerstein, D. Woolfolk, J. R. Doisy: J. clin. Endocr. 24 (1964), 761.

(23) Randle, P. J., C. N. Hales, P. B. Garland, E. A. Newsholme: Lancet 1963/I, 785.

(24) Mehnert, H., A. Fixl: Klin. Wschr. 44 (1966), im Druck. (25) Elrick, H., L. Stimmler, C. J. Hlad, Y. Arai: J. clin. Endocr. 24 (1964), 1076.

(26) McIntyre, N., C. D. Holds worth, D. S. Turner: J. clin. Endocr, 25 (1965), 1317.

(27) Pfeiffer, E. F., M. Telib, J. Amrion, F. Melani, H. Ditschu neit: Dtsch. med. Wschr. 90 (1965), 1663.

(28) Taton, J., D. Pometta, R. A. Camerini-Dávalos, A. Marble: Lancet $1964 /$ II, 1360

(29) Lundbaek, K.: Brit. med. J. 1962/I, 1507.

(30) Nadon, G. W., J. A. Little, W. E. Hall, M. O. O'Sullivan Canad. med. Ass. J. 91 (1964) 1350.

(31) Jahnke, K., F. A. Gries, H Wallenfels, H. Schulte: Klin. Wschr. 42 (1964), 1016.

(32) Conn, J. W., S. S. Fajans Amer. J. Med. 31 (1961), 839

(33) Levine, R.: zit. bei Conn J. W.: Mod. Med. 32 (1964) 130.

(34) Pfeiffer, E. F.: Dtsch. med. Wschr. 90 (1965), 855.

(35) Ditschuneit, H.: Dtsch med. Wschr. 90 (1965), 1925.

(36) Conard, V.: Acta gastroent. belg. 18 (1955), 655; 727; 803.
(37) Fajans, S. S., J. W. Conn: Diabetes 3 (1954), 296.

(38) Sachse, B.: Dtsch. med. Wschr. 89 (1964), 2281.

(39) Butterfield, W. J. H., G. Schless: Diabetes 8 (1959), 450. (40) Werk, E. E., H. C. Know-

les: Diabetes 10 (1961), 22

(41) Schrade, W., E. Böhle, R. Biegler: Dtsch. med. Wschr. 86 (1961), 781.

(42) Schrade, W., E. Böhle, R. Biegler, V. Meder, R. Teicke:

Klin. Wschr. 38 (1960), 126.

(43) Tarrant, M. E., J. Ashmore: Diabetes 14 (1965), 179.

(44) Clayton, R., E. L. Bierman I. L. Schwartz: J. clin. Invest. 38 (1959), 275

(45) Marks, B. H., I. Kiem, A G. Hills: Metabolism 9 (1960) 1133

Anschr.: Dr. P. C. Scriba; Privatdozent Dr. K. Schwarz Dr. G. G. Hofmann, II. Medizinische Klinik der Universität, München 15, Ziemssenstr. 1)
(46) Bottermann, P., K. Schwarz, K. Kopetz: Verh. dtsch. Ges. inn. Med. 71 (1965), 464.

(47) Kopetz, K., P. Bottermann K. P. Eymer, K. Schwarz: Verh. dtsch. Ges. inn. Med. 71 (1965) 762 .

(48) Shafrir, E., A. Gutman: Diabetes 14 (1965), 77.

(49) Mehnert, H.: Dtsch. med Wschr. 89 (1964), 688.

(50) Mehnert, H.: Dtsch. med Wschr. 90 (1965), 785.

(51) Wilkerson, H. L. C.: In: and Treatment, hsg. von Danowski, T. S., Amer. Diabetes Ass., New York 1964, S 31.

(52) McDonald, G. W., G. F. Fisher, C. Burnham: Diabetes 14 (1965), 473 Diabetes Mellitus, Diagnosis 